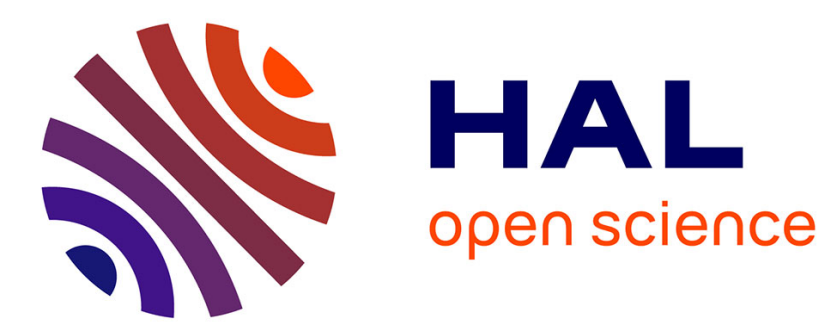

\title{
Charge quantization and Kondo quantum criticality in few-channel mesoscopic circuits
}

\author{
Zubair Qurshi Iftikhar
}

\section{To cite this version:}

Zubair Qurshi Iftikhar. Charge quantization and Kondo quantum criticality in few-channel mesoscopic circuits. Mesoscopic Systems and Quantum Hall Effect [cond-mat.mes-hall]. Université Paris Saclay

(COmUE), 2016. English. NNT: 2016SACLS423 . tel-01482147

HAL Id: tel-01482147

https://theses.hal.science/tel-01482147

Submitted on 3 Mar 2017

HAL is a multi-disciplinary open access archive for the deposit and dissemination of scientific research documents, whether they are published or not. The documents may come from teaching and research institutions in France or abroad, or from public or private research centers.
L'archive ouverte pluridisciplinaire HAL, est destinée au dépôt et à la diffusion de documents scientifiques de niveau recherche, publiés ou non, émanant des établissements d'enseignement et de recherche français ou étrangers, des laboratoires publics ou privés. 
universite்

NNT : 2016SACLS423

\author{
THESE DE DOCTORAT \\ $\mathrm{DE}$ \\ L'UNIVERSITE PARIS-SACLAY \\ PREPAREE A \\ L'UNIVERSITE PARIS-SUD
}

ECOLE Doctorale N ${ }^{\circ} 564$

Physique en Île-de-France

Spécialité de doctorat : Physique

Par

\title{
M. Zubair Iftikhar
}

Quantification de la charge et criticalité quantique Kondo dans des circuits mésoscopiques avec peu de canaux

Thèse présentée et soutenue à Marcoussis, le 21 novembre 2016 :

\section{Composition du Jury :}

M. Cristian Urbina

Mme Julia Meyer

M. Christopher Bäuerle

M. Takis Kontos

M. Serge Florens

M. Frédéric Pierre
Directeur de recherche, CEA-SPEC Professeur, Université Grenoble-Alpes Directeur de recherche, Institut Néel Directeur de recherche, LPA Chargé de recherche, Institut Néel Directeur de recherche, $\mathrm{C} 2 \mathrm{~N}$
Président

Rapporteur

Rapporteur

Examinateur

Examinateur

Directeur de thèse 



\section{Contents}

1 Summary 1

1.1 Charge quantization . . . . . . . . . . . . . . . 2

1.2 Observation of the multi-channel Kondo effect . . . . . . . . . . 6

1.3 Quantum phase transition . . . . . . . . . . . . . . 8

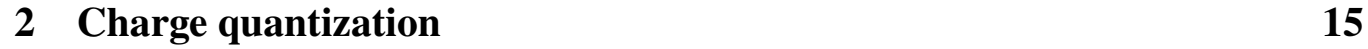

2.1 Previous investigations . . . . . . . . . . . . . . 16

2.1.1 Quantization of the charge in (almost) isolated systems . . 16

2.1.2 Coulomb blockade at almost perfect transmission . . . . . 19

2.2 Theoretical predictions .................. 23

2.2 .1 Transport versus thermodynamic properties . . . . . . . 24

$2.2 .2 \quad$ Asymmetric case $\tau_{L} \ll 1,1-\tau_{R} \ll 1 \ldots \ldots . . . . . . .24$

$2.2 .3 \quad$ Symmetric limit $1-\tau_{L, R} \ll 1 \ldots \ldots . \ldots . . . . .26$

2.2 .4 Universality in the high temperature regime . . . . . . . 28

2.3 Controlling charge quantization . . . . . . . . . . . . 28

2.4 Conclusion $\ldots \ldots \ldots \ldots \ldots$

\begin{tabular}{|lr|}
3 & Multi-channel Kondo effect \\
\hline
\end{tabular}

3.1 The Kondo model: a testbed for correlated physics . . . . . . . . 37

3.1 .1 The original Kondo model . . . . . . . . . . . . . 38

3.1.2 The multi-channel Kondo model . . . . . . . . . . . . . . 44

3.2 The 'charge' implementation of the Kondo model . . . . . . . . . 50

3.2.1 Mapping of the Coulomb blockade Hamiltonian onto the

Kondo model . . . . . . . . . . . . . . 51

3.2.2 Theoretical predictions for the "charge' Kondo model . . . 55

$3.2 .3 \quad$ Experimental implementation . . . . . . . . . . . 60

3.3 Multi-channel Kondo observation . . . . . . . . . . . . 61

$3.3 .1 \quad$ Kondo fixed points . . . . . . . . . . . . . . . 61

3.3 .2 Kondo scaling . . . . . . . . . . . . . . . . . 64

3.4 Conclusion . . . . . . . . . . . . . . . . . . . 68 
\begin{tabular}{|llr}
4 & Quantum phase transition & 69
\end{tabular}

4.1 Quantum phase transition . . . . . . . . . . . . . . . 69

4.1.1 What is a quantum phase transition . . . . . . . 70

4.1 .2 Quantum criticality . . . . . . . . . . . . . 71

4.2 Quantum phase transitions in the Kondo model . . . . . . . . . 76

4.2 .1 Description of the quantum phase transition . . . . . . . 76

4.2.2 Theoretical predictions for the 'charge' Kondo model . . . 79

4.3 Experimental observation . . . . . . . . . . . . . . 83

4.3.1 Development of the Kondo QPT versus channel asymme-

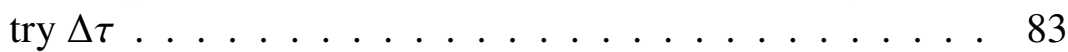

4.3 .2 Unanticipated features of the flow diagrams $\ldots . . . .86$

4.3 .3 Crossover from criticality versus detuning from charge degeneracy $\delta V_{g} \ldots \ldots \ldots \ldots \ldots$. . . . . . . . . . . . . . . . . . 89

4.4 Conclusion $\ldots \ldots \ldots \ldots$. . . . . . . . . . . . . . . . . 92

\begin{tabular}{llr}
\hline 5 & Outlook & 95
\end{tabular}

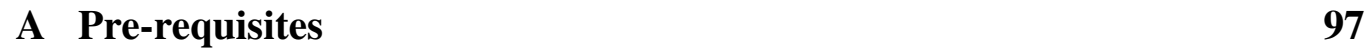

A.1 Quantum conductors . . . . . . . . . . . . . . . . . . . 98

A.1.1 Quantum of conductance . . . . . . . . . . . . . 98

A.1.2 Quantum coherent conductors . . . . . . . . . . . . 100

A.2 Quantum transport of electricity . . . . . . . . . . . . . 102

A.2.1 Quantum Hall effect . . . . . . . . . . . . . . . . 103

A.2.2 Dynamical coulomb blockade (DCB) . . . . . . . . . 105

A.2.3 Quantum shot noise ............... . . 109

\begin{tabular}{lll}
\hline B Sample characterization & 111
\end{tabular}

B.1 Surface gates . . . . . . . . . . . . . . . . . . . . . 112

B.1.1 Switch gates and environment . . . . . . . . . . . 112

B.1.2 $\quad$ Characterization and measurement circuitry . . . . . . . . 114

B.1.3 Quantum point contacts transmission and characterization 115

B.1.4 Capacitive crosstalk corrections . . . . . . . . . . . 117

B.2 Ohmic contact . . . . . . . . . . . . . . . . . 120

B.2.1 Mean energy level spacing in the island . . . . . . . . . 121

B.2.2 Connection of the micron-sized ohmic contact to the 2DEG 121

\begin{tabular}{|rr|}
\hline C Single electron transistor & 123 \\
\hline
\end{tabular}

C.1 Charging Energy . . . . . . . . . . . . . . . . . . . 123

C.2 Coulomb diamonds . . . . . . . . . . . . . . . . . . . 125

C.3 Master equation . . . . . . . . . . . . . . . . . . . . . 126

C.4 Coulomb blockade oscillations . . . . . . . . . . . . . . . . . 128 
\begin{tabular}{|ll}
\hline P Primary thermometry paper & 131
\end{tabular}

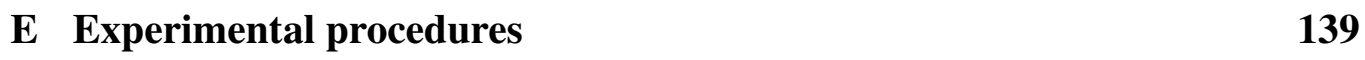

E.1 Extraction of in situ conductances . . . . . . . . . . . . . . . 139

E.1.1 Conductance formulae . . . . . . . . . . . . . . . . 139

E.1.2 Calibration of the gain and the offset . . . . . . . . . . . 141

E.2 Device tuning . . . . . . . . . . . . . . . . . . . . . 142

E.2.1 Symmetric coupling . . . . . . . . . . . . . . . . 142

E.2.2 Transmissions in practice . . . . . . . . . . . . . . 143

E.2.3 Tuning to charge degeneracy $\delta V_{g}=0 \ldots \ldots$. . . . . 144

E.3 Experimental artifacts . . . . . . . . . . . . . . . . . . 147

E.3.1 Systematic peak selection . . . . . . . . . . . . . 147

E.3.2 Crosstalk of the plunger gate . . . . . . . . . . . . . . . . 149

E.3.3 Averaging the selected peaks . . . . . . . . . . . . . . . 149

\begin{tabular}{llr}
\hline Résumé en français & 151
\end{tabular}

F.1 Contrôle de la quantification de la charge . . . . . . . . . . . . . 153

F.2 Observation de l'effet Kondo multi-canaux . . . . . . . . . . . . . 155

F.3 Transition de phase quantique . . . . . . . . . . . 160 


\section{Remerciements}

J'ai toujours été un privilégié. Tout petit, ma maîtresse de maternelle me portait dans ses bras, quand je pleurais, au départ de ma mère. À la maison, alors que mes frères avaient des horaires fixes pour faire leurs devoirs, j'étais libre de travailler quand je voulais. En cinquième, j'ai fait une classe de mer. En quatrième, j'avais une prof de français, Mme Galin, qui nous faisait passer l'agrégation en même temps qu'elle. Au lycée, j'ai eu un prof de maths formidable, M. Squalli, à qui on pouvait même demander "mais pourquoi on n'a pas le droit de diviser par zéro ?". Après le bac aussi, j' ai eu les meilleurs profs. En master, j'ai pu voyager et faire un stage inoubliable en Sibérie.

J'ai grandi dans les meilleurs environnements, avec le meilleur matériel, au contact des meilleures personnes. J'ai eu les meilleurs amis du monde. J'en salue ici quelque uns que je croise encore parfois, par ordre chronologique: Wilfried C. qui partageait son goûter en primaire; Florian B. qui me faisait rire tout le temps; Alexia H. qui se fâchait contre tout le monde sauf moi; Laurent R. avec qui j' ai eu de longues discussions philosophiques au lycée; Alexandre H. que j'ai eu plaisir à voir à ma soutenance de thèse; Ibrahim que je croise à la BU (bon courage); Jérémy D., Thomas N., Maxime J., Jérôme D. mes amis de prépa, merci pour tous les bons moments (les colles, les discussions à la cantine, etc.); Hélène S., Romain J., Arthur L., Dorian G., mes amis à SupOptique; Jérémie D. et tous nos lycéens 'tutorés' avec qui j' ai eu de belles discussions avant, pendant et après les séances; Hamit M., Nicolas M., Charles P., Ana B., Maria O, Anna I. et tous mes amis de Russie.

Et après la Russie, j'ai atterri à Marcoussis, au LPN (aujourd'hui C2N). J'ai beaucoup de personnes à remercier. Tout d'abord, mon directeur de thèse, Fred. J'étais allé le voir un jour pour lui dire que je n'avais rien à faire, il m'a alors répondu "Tu ne dois jamais t'ennuyer!". Et en voyant le désespoir de certains étudiants délaissés par leur encadrant, je me rends compte aujourd'hui que j' ai eu le meilleur directeur de thèse. J'ai passé trois années à un rythme fou. Merci à Sébastien Jézouin, mon 'co-thésard' pendant la première année, qui m'a appris beaucoup de choses, par exemple: courir pour attraper le dernier bus à $20 \mathrm{~h} 09$ juste après avoir lancé 'la manip de la nuit'. Merci à François Parmentier pour 
m'avoir appris le fonctionnement de la manip et du labo, tu es un modèle jusqu'à dans ta façon de parler. Merci Anne Anthore, parce que ta façon de répondre fait que je n'ai jamais eu honte de poser mes questions (souvent idiotes, parfois profondes). Merci aux autres membres de l'équipe en particulier Ulf Gennser pour ta gentillesse et pour avoir relu mon manuscrit en anglais, Yong Jin pour les discussions à la cantine, et Julien Chaste pour avoir toujours été là pour discuter de la physique et de la vie. Merci aux non-permanents du groupe Amina, Khalifa, Hugo et Debora. Je veux remercier aussi tous les autres étudiants et postdocs du LPN, en particuliers ceux avec qui je prenais le bus le soir et que Riadh appelait les habbs: lui-même, Shayma, Avishek, Hakim et les autres. Special thanks to Avishek for the beautiful moments aboard the Titanic. Et pour les parties de foot endiablées du mardi midi, merci à Stéphane, Kamel, Lorenzo, Rolland, Paul, Dominique, Khalifa, Lyas, Mathias, Ivens, Juan, Vivek, Daniel, Amadeo. Qu'il pleuve, qu'il neige, qu'il vente, qu'on soit douze ou seulement huit, on s'est toujours bien amusé! Merci aux étudiants du groupe d'optique Mickaël, Thomas, Fayçal et Benoît pour la bonne ambiance au deuxième étage du D2.

Merci à Christopher Bäuerle et Julia Meyer d'avoir accepté de rapporter ma thèse. Merci à Takis Kontos et Serge Florens pour leurs questions durant la soutenance. Et merci à Cristián Urbina d'avoir présidé mon jury, merci aussi pour la belle discussion qu'on a eue après la soutenance. Je remercie également Gilles Montambaux, directeur-adjoint de l'école doctorale, pour m'avoir rassuré au moment de la rédaction de cette thèse.

Enfin je voudrais remercier ma famille, en particulier ma mère, l'auteur de l'auteur de cette thèse; ma sœur pour m'avoir soutenu avec son humour et mon frère Tahir, parce tu as ouvert la voie à mes études supérieures en me montrant que je pouvais y arriver.

Je me suis posé beaucoup de questions au cours de ma vie et durant ces trois dernières années. J'ai traversé des moments difficiles. J'ai réfléchi, et je pense avoir trouvé. La vie est une promenade dont le seul but est de l'aimer pour ellemême. J'ai atteint ce but, j'aime me balader. Voilà qui explique ma posture de privilégié. Je remercie toutes les personnes que j'ai rencontré sur mon trajet. Celle qui m'accompagne, la main dans la main, et qui a transformé ma balade en ballade. Et enfin, ma maman, qui m'a lancé dans cette aventure il y a vingt-six ans et à qui je dédie cette thèse. 


\section{Preliminary remarks}

The first chapter of this thesis - Chapter 1 - is a summary, we assume that the reader is familiar with quantum conductors and in particular with quantum point contacts (QPCs); otherwise, an introduction to the quantum transport of electricity is provided in Appendix $\mathrm{A}$.

We use the acronyms defined in Table 1 and the symbol ' $\triangleq$ ' for the definition physical or mathematical quantities and functions. The definition of some Greek symbols may change 1 nevertheless the right definition to use is generally obvious.

\begin{tabular}{cc} 
acronym & definition \\
\hline$N-$ CK & $N$-channel Kondo (with $N=1,2 \ldots$ ) \\
2DEG & two-dimensional electron gas \\
CFT & conformal field theory \\
DCB & dynamical Coulomb blockade \\
FL & Fermi liquid \\
FQHE & fractional quantum Hall effect \\
MBE & molecular beam epitaxy \\
NFL & non-Fermi liquid \\
NRG & numerical renormalization group \\
QCP & quantum critical point \\
QHE & quantum Hall effect \\
QPC & quantum point contact \\
QPT & quantum phase transition \\
SET & single electron transistor \\
STM & scanning tunneling microscope
\end{tabular}

Table 1: Definition of the acronyms

${ }^{1}$ For example, $\gamma$ is generally $\gamma \triangleq \exp C$, with $C \approx 0.5772$ the Euler's constant. But sometimes $\gamma$ refers to a generic critical exponent. 


\section{Chapter 1}

\section{Summary}

This thesis reports electrical transport measurements of a tunable and characterizable nano-device (shown in Fig. 1.1). Owing to its hybrid metal-semiconductor structure and to multiple gates, this device provides a quantitative testbed to strongly correlated and critical physics. It can be tuned to a regime where a few independent electronic channels are strongly interacting with the 'charge' macroscopic quantum degrees of freedom of the metallic node (in purple in Fig. 1.1) of the circuit. We used this sample to probe and to control the degree of charge quantization on a metallic node, depending on its connection to other conductors. The device can also be tuned to implement the multi-channel Kondo model and perform reliable quantum simulations of this quantum many-body model. As such, it provides an experimental testbed for some of the most powerful quantum many-body techniques (the numerical renormalization group [131, 20, 113], Bethe ansatz [117, 13], conformal field theory [116, 3, 4] or bosonization [39, 78, 43]).

We have observed good agreements between our experimental data and the predicted universal power laws both near the strong coupling limit in the problem of the charge quantization and in the vicinity of the quantum critical points of the multi-channel Kondo effect. The crossover from these critical points reveals intriguing physics, in particular at intermediate temperature. The approach developed in this thesis paves the way to further study of the tantalizing non-Fermi liquids physics underlying the field of strongly correlated materials.

The remainder of this introductory chapter gives a quick preview of the results reported in this thesis, with each section corresponding to a chapter. We start with a description of our single-electron device and answer a long standing question on the criterion of how the charge quantization is destroyed by the quantum fluctuations in such a device. In the next section, we will see that this system can be mapped onto the Kondo model using the 'charge' degrees of freedom of the circuit. Under the renormalization process, its conductance is predicted to flow towards non-trivial Kondo fixed points at low temperature. In the next and last section, we focus on the 


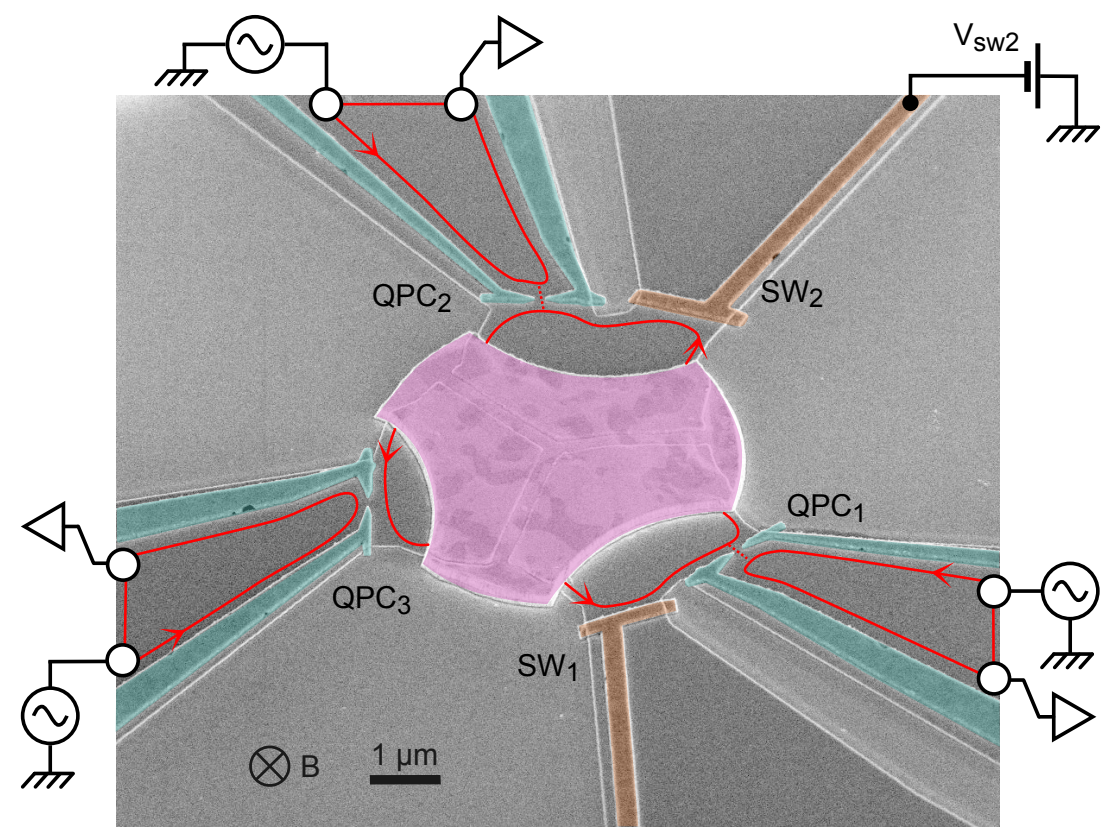

Figure 1.1: Colored micrograph of the sample with measurement schematics. This figure shows the central ohmic contact (in purple) that redistributes the current injected by the a.c. voltage sources (out of the image) into the chiral edge states of the IQHE (red lines) through larger ohmic contacts (white circles) not shown in the picture. The low frequency signals are measured using Lock-in amplifiers (triangles). This sample includes three QPCs (in cyan) and two switch gates (in orange) used for characterization. The value of the transmission of the QPC and the state of the switch is controlled by the voltage sources that connect (black circles) theses surface gates.

quantum critical scalings that occur when we purposely introduce perturbations that are 'relevant' in the renormalization group sense.

\subsection{Control of the charge quantization}

\section{A single-electron transistor in the strong coupling limit}

The central piece of metal shown in purple in Fig. 1.1 is the main character of this thesis. It will be called the island, since it is connected to the circuit by only a few electronic channels. The geometry of the sample sets the typical charging energy $E_{C} \triangleq e^{2} /(2 C)$ (with $e$ the elementary charge and $C$ the geometrical capacitance of the island) required to add/remove an electron to/from the island. Singleelectron effects are thus important at temperatures and energies below this scale: 
$k_{B} T, e V \ll E_{C} \approx 25 \mu \mathrm{eV} \approx k_{B} \times 300 \mathrm{mK}$.

The connection between the island and the electronic circuit (constituted of large electronic reservoirs) can be adjusted through tunable QPCs (in cyan). Here we consider only two QPCs (see Fig. 1.2 a). When the transmissions of both left and right QPCs are set to the tunnel regime $\tau_{L, R} \ll 1$, the island is only weakly connected to the surroundings and its charge is quantized in units of $e$. In this limit, the device implements the well-known single-electron transistor (SET). The number of charges on the island can be tuned separately by sweeping the voltage $V_{g}$ of a lateral gate (e.g. a characterization gate, in orange in Fig. 1.1).
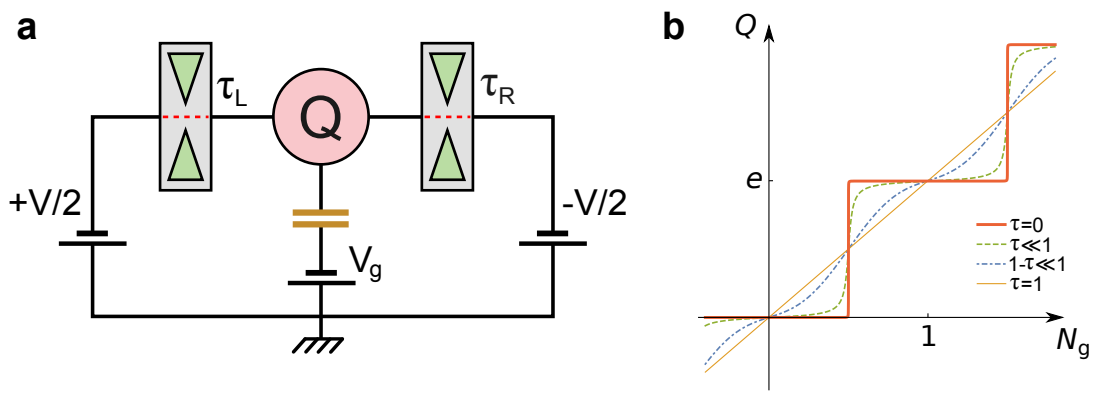

Figure 1.2: Charge quantization in a single-electron device. a, Schematics of the sample. The differential conductance $G_{\mathrm{SET}}$ is measured by applying an a.c. voltage bias to the device. In presence of charge quantization, this conductance will show Coulomb oscillations with respect to a capacitively coupled gate $V_{g}$. b, (adapted from [78]) The average charge $Q$ on the island is plotted at zerotemperature versus $N_{g} \triangleq V_{g} / \Delta V_{g}$ (where $\Delta V_{g}$ is the period of the Coulomb oscillations) for different connection strength $\tau$ (the transmission probability $\tau \triangleq$ $\tau_{R}$, with $\tau_{L}=0$ ). The charge quantization completely disappears as soon as there is one ballistic electronic channel connected to the island $(\tau=1)$.

One would then observe Coulomb blockade oscillations of the conductance of the device $G_{\mathrm{SET}}\left(V_{g}\right)$ as current is allowed only when two successive charge states of the island are degenerate in energy (up to thermal fluctuations, otherwise the charge state is frozen and the current is blocked). The number of electrons on the island is thus incremented by one after each peak of conductance shown in Fig. 1.3 a. In this figure, we see that the oscillations progressively vanish as the transmission of a QPC tends to unity $\tau \longrightarrow 1$. A more systematic study consists in plotting the visibility of the oscillations $\Delta Q \triangleq\left(G_{\mathrm{SET}}^{\max }-G_{\mathrm{SET}}^{\min }\right) /\left(G_{\mathrm{SET}}^{\max }+G_{\mathrm{SET}}^{\min }\right)$ as a function of $\tau_{R}$. All of the traces obtained for different values of a fixed $\tau_{L}$ collapse when the transmission becomes ballistic at $\tau=1$, and no Coulomb oscillation is observed above this limit (see Fig. 1.3p).

It is known theoretically that quantum fluctuations of the charge smear the charge quantization (even at zero temperature) [48]. In 1995, Matveev showed 
a
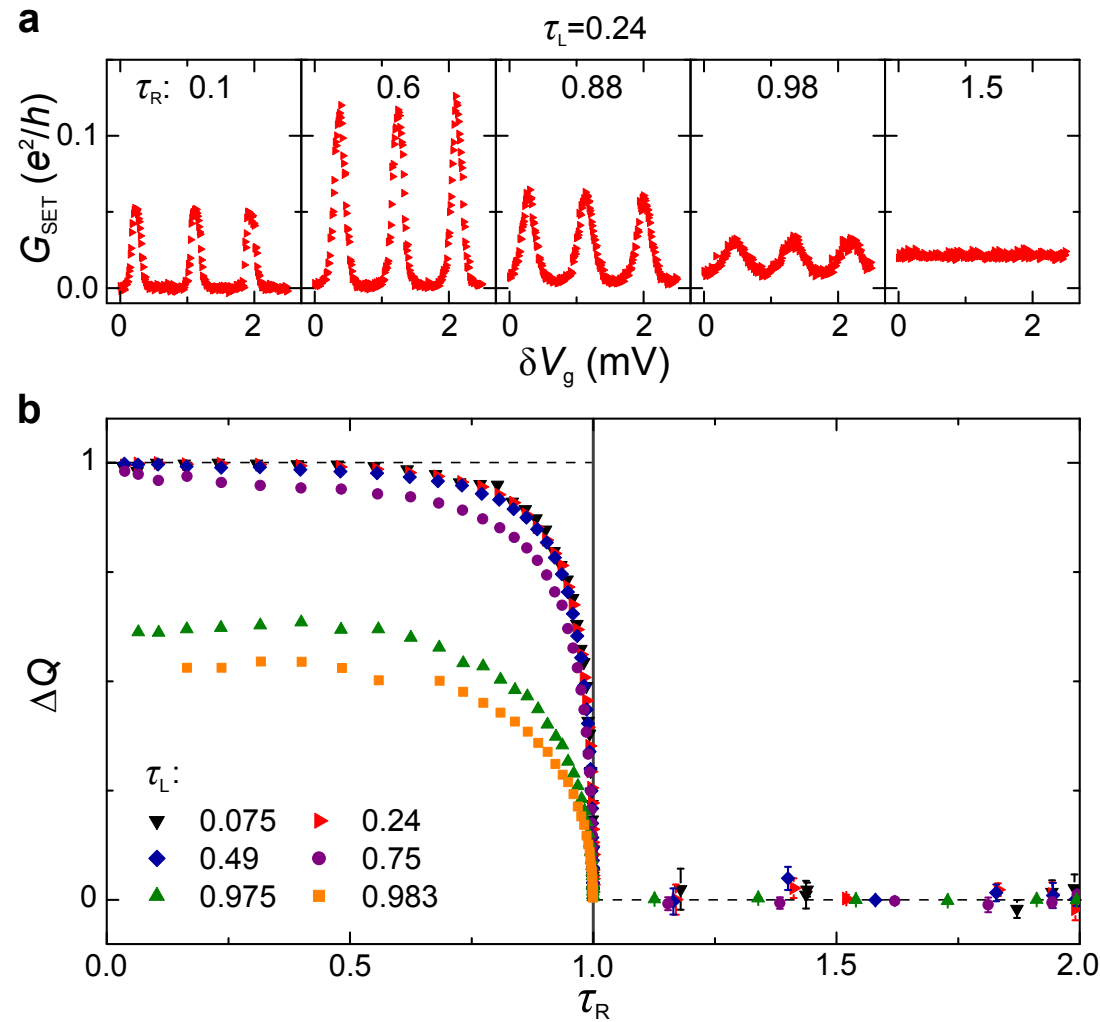

Figure 1.3: Charge quantization versus connection strength at $T \simeq 17 \mathbf{m K}$. a, Conductance sweeps $G_{\mathrm{SET}}\left(\delta V_{\mathrm{g}}\right)$ with a fixed $\tau_{L}=0.24$, and $\tau_{R}=0.1,0.6$, $0.88,0.98$ and 1.5 , from left to right respectively. $\mathbf{b}$, Visibility of $G_{\mathrm{SET}}$ oscillations versus $\tau_{R}$, with each set of symbols corresponding to a different $\mathrm{QPC}_{L}$ set-point.

that quantization is completely destroyed in the ballistic limit when considering an island with a continuous density of states (see Fig. 1.2p) [78]. In 1993, a controversy arose regarding this criterion for the destruction of charge quantization, as some experimentalists validated it [119] whereas others observed Coulomb oscillations above the ballistic limit [100]. But these early experiments were based on non-metallic islands with discrete density of states, and where the phase coherence of the electrons could give rise to subtle mesoscopic effects [5]. The design and the materials we used to fabricate our sample avoid any such coherent effects, since when an electron enters the island, it stays there for much longer than its quantum phase coherence time. 


\section{Quantitative comparison with theory}

Assuming a continuous density of state in the island and spinles 1 electrons, quantitative predictions can be established for this system in several limits. At low temperature $k_{B} T \ll E_{C}$, we can compare the visibility $\Delta Q$ of the conductance oscillations when approaching the ballistic limit $\tau_{R} \longrightarrow 1$ with the quantitative theory in two limits (strong coupling $1-\tau_{L} \ll 1[43]$ and asymmetric $\tau_{L} \ll 1$ [80]). We are able to characterize all the parameters $\left(\tau_{L}, \tau_{R}, E_{C}\right.$ and $\left.T\right)$ independently, and the quantitative agreement found in the strong coupling limit is therefore established without any fitting parameters (see Fig. 1.4a).
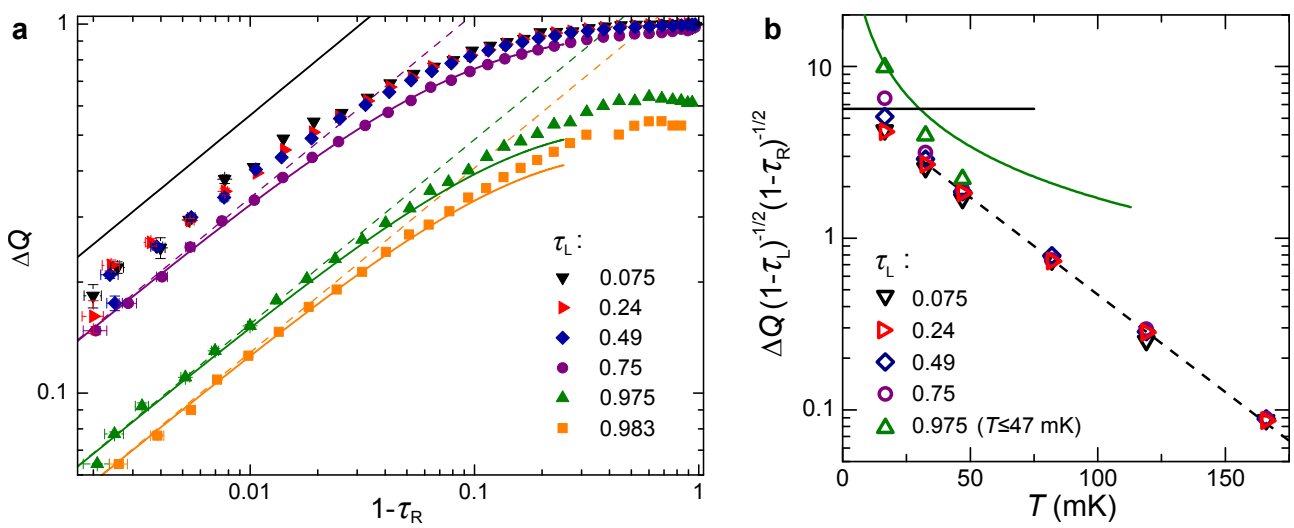

Figure 1.4: Charge quantization scaling near the ballistic point and its exponential suppression with temperature. a, The visibility $\Delta Q$ of the Coulomb oscillations at $T \approx 17 \mathrm{mK}$ is displayed versus $1-\tau_{R}$ in a $\log -\log$ scale, with distinct sets of symbols for the different $\mathrm{QPC}_{L}$ set-points. Solid lines are quantitative predictions (no fit parameters) derived assuming $k_{B} T \ll E_{C}, 1-\tau_{R} \ll 1$ and either $\tau_{L} \ll 1$ (top straight line) or $1-\tau_{L} \ll 1$ (three bottom continuous lines). The power law $\Delta Q \propto \sqrt{1-\tau_{R}}$ (straight dashed lines) is systematically observed for $1-\tau_{R} \lesssim 0.02$, also at intermediate $\tau_{L}$. b, Symbols display versus $T$, in semilog scale, the rescaled data $\Delta Q / \sqrt{\left(1-\tau_{R}\right)\left(1-\tau_{L}\right)}$, extracted in the regime of small enough $1-\tau_{R}$ such that $\Delta Q \propto \sqrt{1-\tau_{R}}$. Solid lines correspond to the quantitative predictions in the quantum regime $k_{B} T \ll E_{C}$ shown in a. The straight dashed line displays an exponential decay close to predictions in the presence of strong thermal fluctuations.

In the two limits, the theory predicts a $\sqrt{1-\tau_{R}}$ dependence for the visibility of the Coulomb oscillations. This power law behavior has been observed also for intermediate transmissions of $\tau_{L} \in[0,1]$. At higher temperatures, this dependence becomes completely universal on the full range $\tau_{R, L} \in[0,1]$ (as indicated by the collapse of all the $\tau_{L}$ data points in Fig. 1.4b). Moreover, in Fig. 1.4b, we note

${ }^{1}$ The spin degeneracy is lifted with a strong magnetic field. 
that the charge quantization (measured by $\Delta Q$ ) is also exponentially suppressed with the temperature $T$, as expected by the theory in presence of strong thermal fluctuations $k_{B} T \gg E_{C} / \pi^{2}$ ([55] and references therein).

\subsection{Observation of the multi-channel Kondo effect}

\section{The original Kondo model and some of its variations}

In 1964, Kondo computed the contribution to the resistivity of the scattering of conduction electrons by magnetic impurities in dilute alloys [62]. However, his perturbative approach fails at low temperatures compared to the so-called Kondo temperature $T_{K}$. This is a typical problem suited for the renormalization group theory. Its first exact solution was found by Wilson using numerical renormalization group [131].

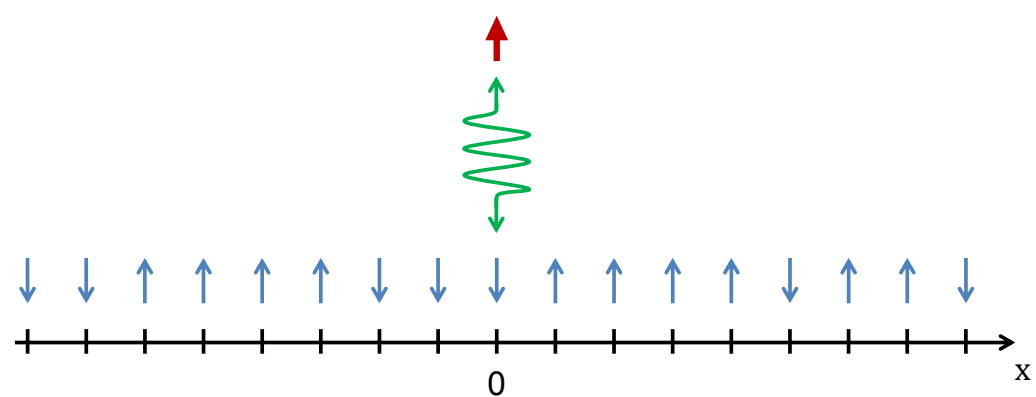

Figure 1.5: Diagram explaining the Kondo model. The impurity $S=1 / 2$ is represented by a red arrow. The conduction electrons (blue arrows) are distributed on a lattice. To simplify, the lattice is 1D. The antiferromagnetic interaction is drawn as a squiggly green arrow on the impurity site.

The original Kondo model is illustrated in Fig. 1.5. It describes a magnetic impurity (modeled by a spin $\vec{S}, S=1 / 2$ throughout this thesis) that interacts with a single band of conduction electrons (shown as a 1D lattice) through an on-site local antiferromagnetic coupling $J$. At low temperature $J$ is effectively renormalized to $J \longrightarrow \infty$ [131]. In this limit, the impurity forms a singlet with an electron, and this complete screening yields a simple Fermi liquid description [94]. Nozières and Blandin proposed a generalization of the model with $N$ independent electronic channels [93], which leads to non-Fermi liquid ground state at low temperature in the overscreened case $N>2 S$. In the renormalization group picture, the couplings $J_{i}$ between the quantum impurity and each electronic channel are effectively renormalized as the temperature is lowered [10]. At zero-temperature, 
they eventually reach a universal Kondo fixed point, which depends on the number of channels $N$ [93].

In our device, two successive charge states can be tuned (using $V_{g}$ ) to have the same energy. The number of electrons on the island $n$ or $n+1$ can then play the role of a quantum two-level system, or, in other words, of a pseudo-spin $S=1 / 2$. In 1991, Matveev demonstrated, in the weak coupling limit, the exact mapping between the original 'spin' Kondo model and the Coulomb blockade that models our system [79]. This mapping, which is also valid beyond the tunnel limit [78, 72], involves the coupling between the Kondo 'charge' pseudo-spin and a localization pseudo-spin of the electrons (either in or out of the island).

\section{Observation of multi-channel Kondo effects and universal be- haviors}

The conductance $G_{i}$ of each QPC will be renormalized towards the 'charge' Kondo fixed points at low temperature. In Fig. 1.6, when we tune all the transmissions to be in use equal ( $\tau \triangleq \tau_{1}=\tau_{3}$ and $\tau_{2}=0$ for two-channel (2CK); $\tau \triangleq \tau_{1}=\tau_{2}=\tau_{3}$ for three-channel $(3 \mathrm{CK}))$ and the gate voltage to charge degeneracy $\left(\delta V_{g}=0\right)$, we observe that the conductance flows to the predicted 'charge' Kondo fixed point (which is extremal $G_{2 C K}^{*}=e^{2} / h$ for two-channel and intermediate $G_{3 C K}^{*}=$ $2 \sin ^{2}(\pi / 5) e^{2} / h \approx 0.691 e^{2} / h$ for three-channel) [135]. Note that these fixed points are universal, i.e. they do not depend on any microscopic parameters (transmission $\tau$, charging energy $E_{C}$, etc.).

The scaling and universal properties are inherent to renormalization [131]. In the Kondo model, the temperature evolution of any observable is a universal function of the rescaled temperature $T / T_{K}$, provided the temperature has been lowered enough so that the renormalization has suppressed the influence of the 'irrelevant' perturbations [92]. Mitchell and co-workers have computed the full universal curve of conductance $G\left(T / T_{K}\right)$ (from $G\left(T / T_{K} \gg 1\right) \approx 0$ to $G\left(T / T_{K} \ll\right.$ $1) \approx G^{*}$ ) for both the two- and three-channel 'charge' Kondo models [85, 51]. We compare our experimental data to their exact numerical calculations in Fig. 1.7. Our procedure is to rescale the temperature to match the point at base temperature with the theoretical curve. We note that at least the three lowest temperature points are in the universal regime as they follow the theoretical curve. This procedure works on many orders of magnitude in $T / T_{K}$, highlighting the outstanding tunability of our sample. At higher temperatures, non-universal effects appear. In fact, this crossover to a non-universal regime is fully explained by numerical renormalization group calculations taking into account the finite charging energy $E_{C}$. From this rescaling in $T / T_{K}$, one can also extract the relation between the Kondo temperature $T_{K}$ and the unrenormalized coupling strength $\tau$. In the insets of Fig. 1.7, we 

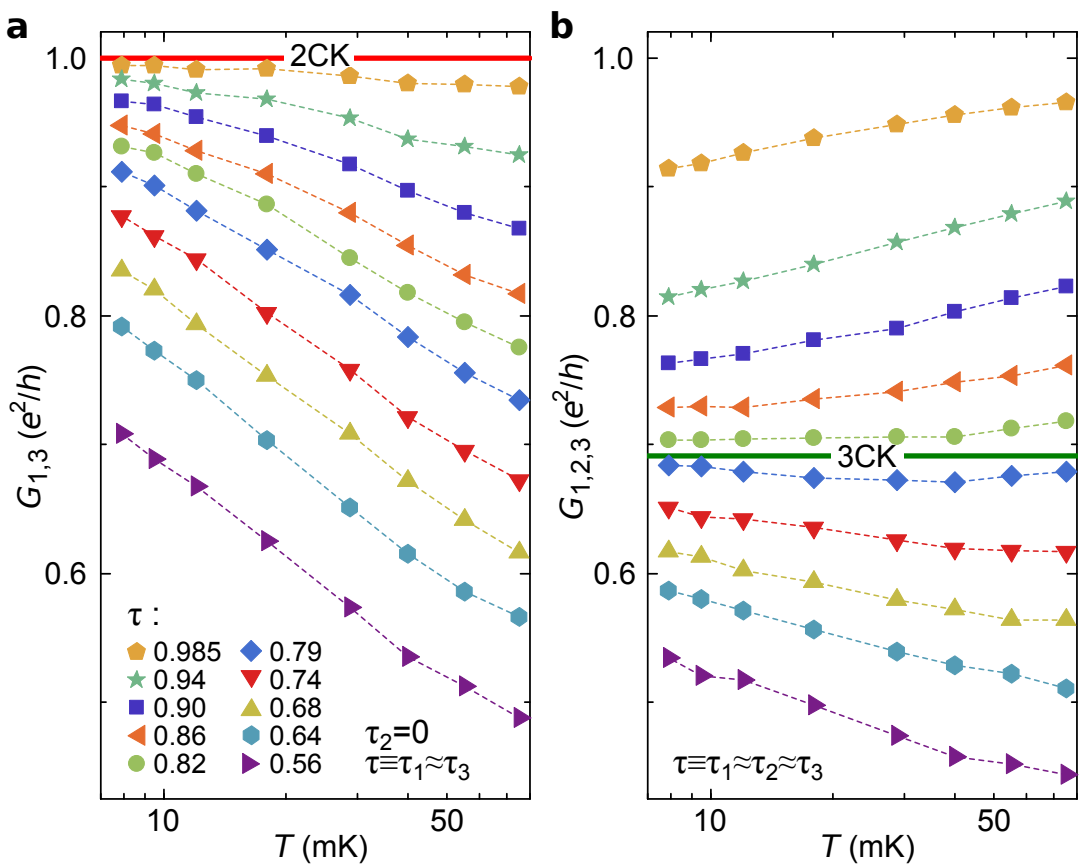

Figure 1.6: Renormalization towards the two- and three-channel 'charge' Kondo fixed point. For the same set of transmissions $\tau$, the individual conductances at degeneracy $\delta V_{g}=0$ are plotted versus the temperature $T \approx\{7.9,9.5,12$, $18,29,40,55,75\} \mathrm{mK}$ in log-scale. a, When lowering the temperature, the conductance flows towards the $2 \mathrm{CK}$ fixed point (red thick line) for all the transmissions in the case of two symmetric channels $\left(\tau_{1} \approx \tau_{3}\right)$. b, With three symmetric channels, it flows to $G_{3 \mathrm{CK}}^{*}$ (green thick line).

compare $T_{K}$ to the theories near the fixed points and in the tunnel regime.

\subsection{Quantum phase transition in multi-channel Kondo systems}

\section{Quantum criticality}

The work on quantum phase transitions has been mainly motivated by one of the most important unsolved problem in condensed matter physics, which concerns the complicated phase diagram of some strongly correlated materials. In particular, the 'strange metal' phase from which the superconductivity of high- $T_{C}$ superconductors emerges at low temperature has attracted a lot of theoretical effort but remains poorly understood (see [59] for a recent review and Fig. 1.8 a). This phase displays signatures of quantum criticality such as a non-Fermi liquid power law 

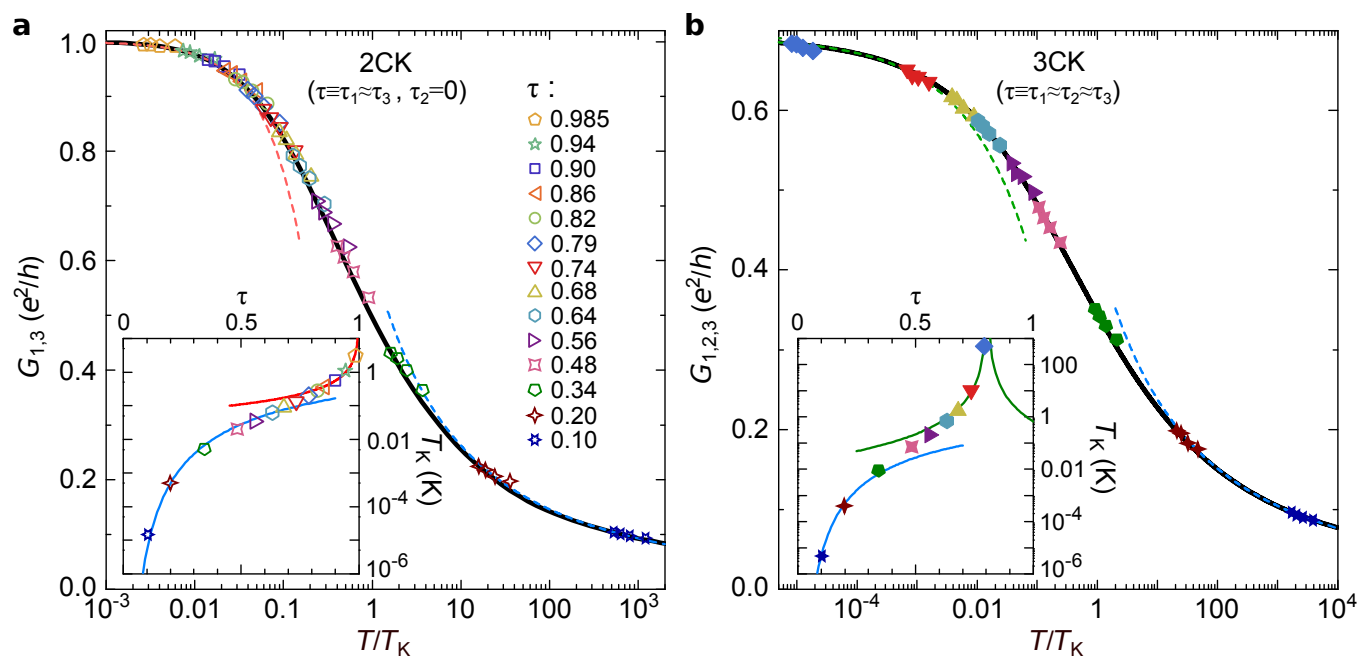

Figure 1.7: Comparison of the experimental data to the universal curves of the conductance for the two- and two-channel 'charge' Kondo model. Here, experimental data are displayed up to $T=29 \mathrm{mK}$ and some additional transmissions are shown as compared to Fig. 1.6 Each set point at $\tau$ fixed is shifted horizontally in the semi-log representation so that the lowest temperature point matches the theoretical curve (solid black line). This defines a scaling temperatures $T_{K}(\tau)$ that are plotted in the insets for both the $2 \mathrm{CK}(\mathbf{a})$ and $3 \mathrm{CK}(\mathbf{b})$ configurations and compared to theoretical predictions (colored lines). The blue lines in the tunnel regime correspond to a perturbative theory [10, 43]. The colored dashed lines are the predicted power laws for the conductance near the Kondo fixed points [43, 51].

of the resistivity versus temperature and a widening of the critical interval with temperature.

In contrast to classical critical phenomena that occur at the critical temperature $T_{C}$ of a second-order phase transition, quantum criticality originates from quantum fluctuations that exist even at zero temperature at a quantum critical point. A physical system is driven to quantum criticality thanks to a non-thermal parameter $g$ (e.g. the doping as in Fig. $1.8 \mathrm{a}$, the pressure, or a gate voltage). It will exhibit quantum criticality for a range of parameter that starts from $g=g_{c}$ at zerotemperature, and which widens as a power law $T_{\mathrm{co}} \propto\left(g-g_{c}\right)^{\gamma}$, where $\gamma$ is called 'critical exponent' and $T_{\text {co }}$ is the crossover temperature below which the system escapes quantum criticality (see Fig. 1.8p) [107, 125].

Despite the opportunity offered by tunable nano-devices for comparisons with theory, the realizations of quantum criticality in such systems are rare [81, 82, 60]. Exact predictions exist for the overscreened Kondo fixed points, which are known for their non-trivial critical exponents on various physical quantities ([3, 4] and references therein). In our 'charge' implementation, we can observe a 


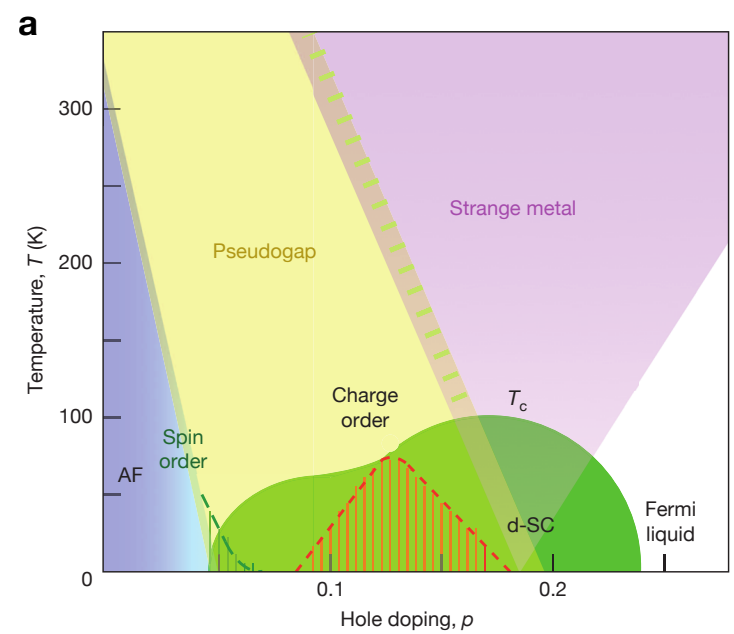

b

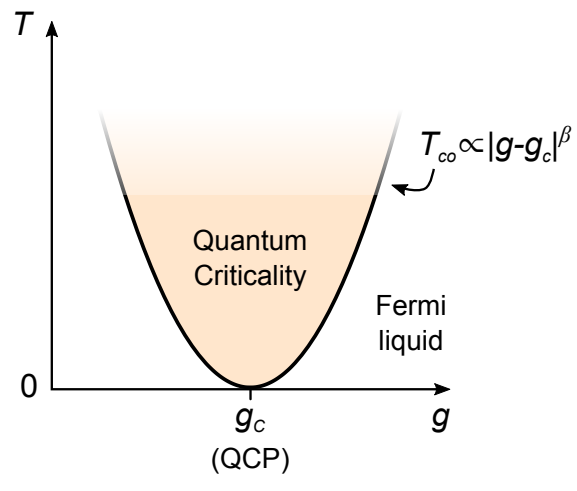

Figure 1.8: Typical phase diagrams. a, (reproduced and simplified from [59]) Typical phase diagram of a copper oxide plotted versus hole doping. At low temperature, depending on the hole doping, these systems display either an antiferromagnetic order (AF, in blue), a spin order (green strips), a charge order (red strips) or a $d$-wave superconducting order (d-SC, in green). A Fermi liquid is obtained in the overdoped regime at low temperature. b, Typical phase diagram displaying quantum criticality when the non-thermal parameter $g$ is tuned around the quantum critical point (QCP) at low temperature.

crossover from quantum criticality by either introducing a channel asymmetry on the transmissions $\tau$ or by detuning from charge degeneracy $\delta V_{g}=0$.

\section{Development of a quantum phase transition}

In Fig. 1.9 we set $\delta V_{g}=0$, and we plot the conductance $G$ versus temperature. Each arrow points towards low temperatures and corresponds to a given configuration $\left(\tau_{2} ; \tau_{1}=\tau_{3}\right)$. This graph therefore provides a visualization of the three-channel Kondo renormalization flow (with two channels set symmetric $\tau_{1}=\tau_{3}$ ). Depending on the number of symmetric channels that share the largest bare connection $\tau$ with the island, the individual conductances $G$ flow towards one of the one- (blue disk), two- (red disk) or three-channel (green disk) Kondo fixed points.

For symmetric transmission configurations $\tau_{1}=\tau_{2}=\tau_{3}$, along the diagonal, the individual conductances $G$ remain symmetric at all temperatures. However, this setting is visibly critical since any initial asymmetry grows as the temperature is lowered. This graph therefore provides a direct view on the development of a quantum phase transition.

In the weak coupling regime $\tau_{1,2,3} \ll 1$, where all the channels can be treated independently and with a perturbative theory [10], an increase of the conductances 


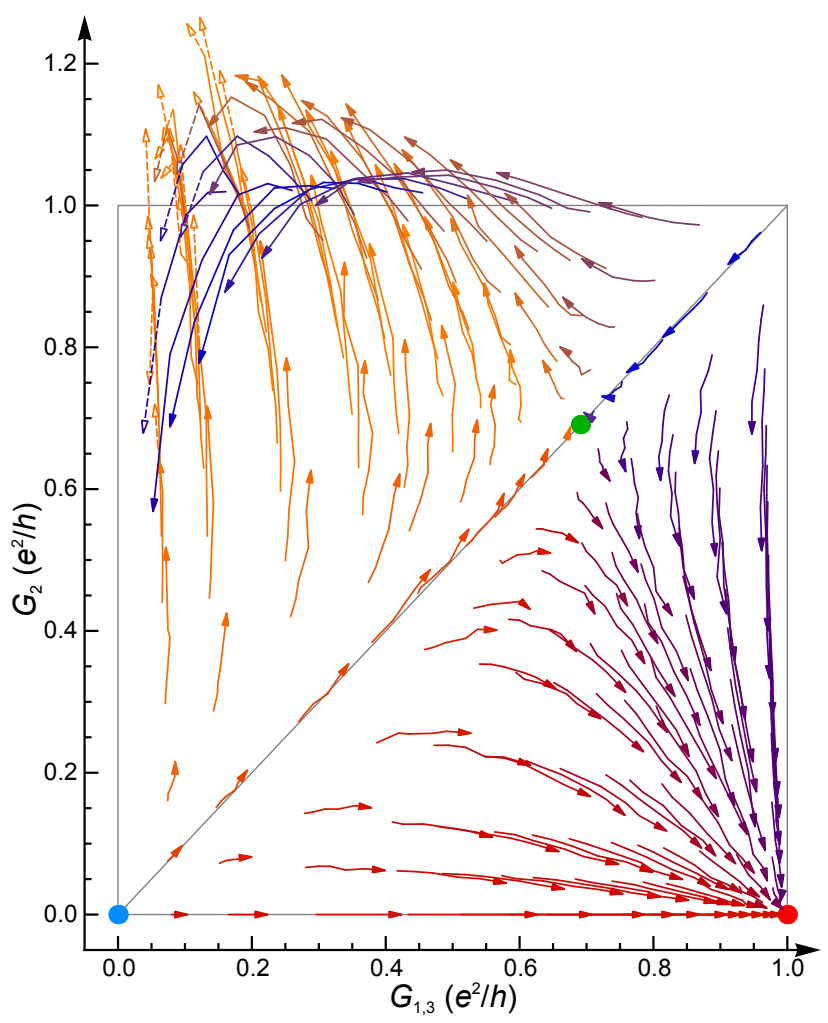

Figure 1.9: Observation of the $3 \mathrm{CK}$ renormalization flow diagram. The averaged conductance of $G_{1}$ and $G_{3}$ is plotted versus $G_{2}$ at temperatures $T \approx\{7.9$, $9.5,12,18,29,40,55\} \mathrm{mK}$. The color of the arrows maps their orientation. The uncertainty on the open symbols and dashed lines is smaller than $0.1 e^{2} / h$ whereas it is smaller than $0.05 e^{2} / h$ for the solid symbols and lines. The Kondo fixed points are indicated with colored dots (1CK in cyan, $2 \mathrm{CK}$ in red and $3 \mathrm{CK}$ in green).

is predicted [43]. The position of the one-channel fixed point at $J_{1 \mathrm{CK}}^{*} \longrightarrow \infty$ in the 'spin' Kondo effect corresponds unexpectedly to $G_{1 C K}^{*}=0$ for our device. This yields the non-monotonic behaviors and the arrow crossings visible in the upper part of the diagram $\left(\tau_{2}>\tau_{1,3}\right)$.

A remarkable overstepping of the quantum of conductance by the individual conductance of a single channel is also observed in the flow towards the one-channel fixed point. This observation has been corroborated by the recent numerical renormalization group calculations of A.K. Mitchell. Another important feature of this diagram is the visualization of the crossover from the $3 \mathrm{CK}$ non-Fermi liquid fixed point to the $2 \mathrm{CK}$ non-Fermi liquid fixed point. 


\section{Crossing over from quantum criticality using an effective mag- netic field}

In this section, we set symmetric transmissions (either $\tau \triangleq \tau_{1}=\tau_{3}$ and $\tau_{2}=0$ or $\tau \triangleq \tau_{1}=\tau_{2}=\tau_{3}$ ). The fragile non-Fermi liquid quantum critical state obtained at low temperature $T \ll T_{K}, E_{C}$ is destroyed by detuning the device from charge degeneracy. A non-zero $\delta V_{g}$ would favor one state of the charge pseudo-spin and destroy the Kondo effect as an effective magnetic field [79]. This would drive the system to a Fermi liquid state with a typical energy scale given by the crossover temperature $T_{\text {co }}$ (see Fig. 1.8p). This quantity is predicted to depend on $\delta V_{g}$ as a power law $T_{\mathrm{co}} \propto \delta V_{g}^{\beta(N)}$ with a critical exponent $\beta(N)=(2+N) / N$ [29].
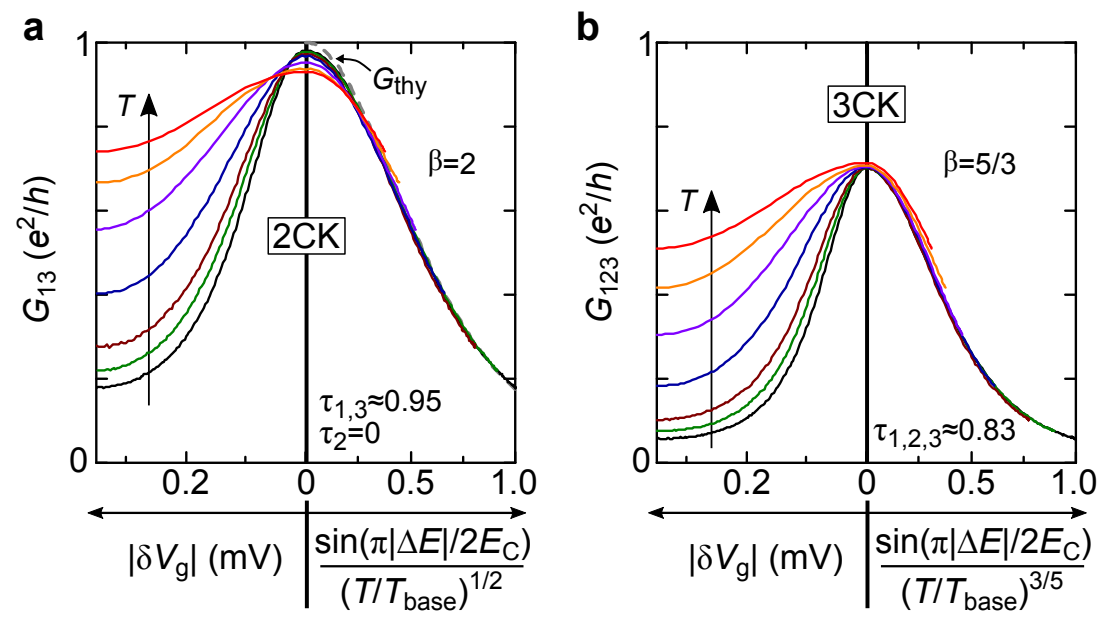

Figure 1.10: Crossover from quantum criticality when detuning from charge degeneracy. A peak of conductance is shown at $T \approx\{7.9,9.5,12,18,29,40$, $55 \mathrm{mK}$ for a selected transmission $\tau$ close to the $2 \mathrm{CK}$ (in a) and the $3 \mathrm{CK}$ (in b) fixed points. The solid lines are experimental data. For each graph a and b, two scales are used: the raw plunger gate voltage $\delta V_{g}$ (left) and a rescaled axis $\sin \left(\pi \Delta E /\left(2 E_{C}\right)\right) /\left(T / T_{\text {base }}\right)^{1 / \beta}$, with $T_{\text {base }}=7.9 \mathrm{mK}$ (right). The conductance adopts a universal behavior in the rescaled axis (except for the highest two temperatures). The $G_{\text {thy }}$ gray dashed line in a shows the zero temperature prediction for $\tau=0.95$.

Moreover, in the vicinity of the quantum critical point, $T_{\text {co }}$ is the only energy scale to consider, and the conductance is expected to follow a universal function of $T / T_{\mathrm{co}}[102,85]$. The quantitative expression of $T_{\mathrm{co}}(\Delta E)$ with respect to the level splitting $2^{2} \Delta E$ have been computed by Furusaki and Matveev for the 'charge' $2 \mathrm{CK}$ model for arbitrary $\Delta E$ (beyond the power law at $\Delta E \ll E_{C}$ ) [43]. In Fig. 1.10a,

${ }^{2}$ As explained above, $\delta V_{g}$ acts as a magnetic field. We define $\Delta E \triangleq 2 E_{C} \delta V_{g} / \Delta V_{g}$, where $E_{C} / k_{B} \approx 300 \mathrm{mK}$ is the charging energy and $\Delta V_{g} \approx 0.70 \mathrm{mV}$ is the period of the Coulomb 
we use this expression to rescale $\delta V_{g}$ and we observe that the conductance peaks for different temperatures all collapse on the same universal curve. Knowing the critical exponent $\beta(N)$, we have proposed a naive generalization of this rescaling that yields a collapse of our 3CK conductance peaks (see Fig. 1.7p). We therefore verify the predicted universal power law for the crossover temperature $T_{\text {co }}$ at small $\delta V_{g}$ and we demonstrate a quantitative agreement with the full theoretical prediction of Furusaki and Matveev (dashed line in Fig. 1.10a) [43].

blockade oscillations (these two numerical values are determined by the geometry of the sample and are independent of $T$ or $\tau$ ). 


\section{Chapter 2}

\section{Charge quantization}

In this chapter, we address the very basic problem of how the quantization of the charge as a multiple of the elementary electron charge ${ }^{1} e$ evolves when an isolated conductor called island is progressively connected to an electron reservoir.

In our experiment, the island consists of a micron-size piece of metal. When the island is weakly connected to an electronic reservoir (e.g. by a tunnel junction), the electrons wave functions remain localized on the island and the charge quantization is preserved. As the connection to the reservoir is increased, due to quantum fluctuations, the wave functions spread out of the island and the charge quantization on the island is progressively reduced. Eventually, when the island is perfectly connected to a reservoir the number of charge localized on the island can no longer be defined: charge quantization is destroyed. Thus, having tunable and wellcharacterized contacts to the island provides a knob to control the degree of charge quantization. In practice this is achieved by using single electronic channels made in a semiconductor.

In the first section of this chapter we will present previous experimental and theoretical investigations on charge quantization. In particular, we will discuss conflicting experiments regarding the criterion to destroy completely charge quantization. The second section is devoted to the quantitative theoretical predictions on the degree of charge quantization versus the connection strength and the temperature. In the last section, we will reprint our latest article on the charge quantization issue.

${ }^{1}$ The value given by the NIST (http://physics.nist.gov/cgi-bin/cuu/Value?e) for this constant is: $e=-(1.6021766208 \pm 0.0000000098) \times 10^{-19} \mathrm{C}$. 


\subsection{Previous investigations on charge quantization}

The quantization of the charge on an isolated system is quite an old topic. After the seminal experiment of Millikan in 1909, nanofabrication has allowed to build the first single-electron practical devices in the eighties. Relying on charge quantization, these single-electronics devices have applications in metrology, for charge detection or temperature measurement.

This section mixes the presentation of early experimental investigations and theoretical explanation of these observations. It is divided in two subsection: first, we discuss almost isolated systems; and second, we consider islands that are almost perfectly connected to an electrical circuit.

\subsubsection{Quantization of the charge in (almost) isolated systems}

We quickly present Millikan's experiment of isolated oil drops. Then we turn to nano-devices embedding almost isolated parts called island and explain the criteria to have a well quantized charge on the island.

\section{Millikan's oil drop experiment}

The most famous experiment on charge quantization is probably the seminal work of Millikan [84] led in 1909 where he measured speed of oil drops subject to a constant electric field $\vec{E}$ created between two electrodes. The electrostatic force $q \vec{E}$ directly depends on the excess charge $q$ of the oil drop (which can be ionized with X-rays). It has been observed that the excess charge $q$ is a multiple of a fundamental constant $e$ which can be estimated from the knowledge of the viscosity of air and other parameters. In this experiment, the drops are isolated systems that can carry only an integer number of excess charge (see Fig. 2.1).

\section{Conditions for a well-quantized charge state in nanostructures}

Single-electron effects can be observed in nanofabricated devices that contain weakly connected parts called islands. The geometrical shape of an island determines its capacitance $C$. The smaller the island, the harder it is to add (or remove) an extra electron. Such an operation typically costs the charging energy $E_{C} \triangleq e^{2} /(2 C)$. Charging effects are best visible when the thermal energy $k_{B} T$ and the voltage bias $V_{\mathrm{dc}}$ are negligible compared to the charging energy $E_{C}$ :

$$
k_{B} T, e V_{\mathrm{dc}} \ll E_{C}
$$

In principle, charging effects remain measurable up to the charging energy $E_{C}$. A simple criterion on the weakness of the connection to ensure that charge 


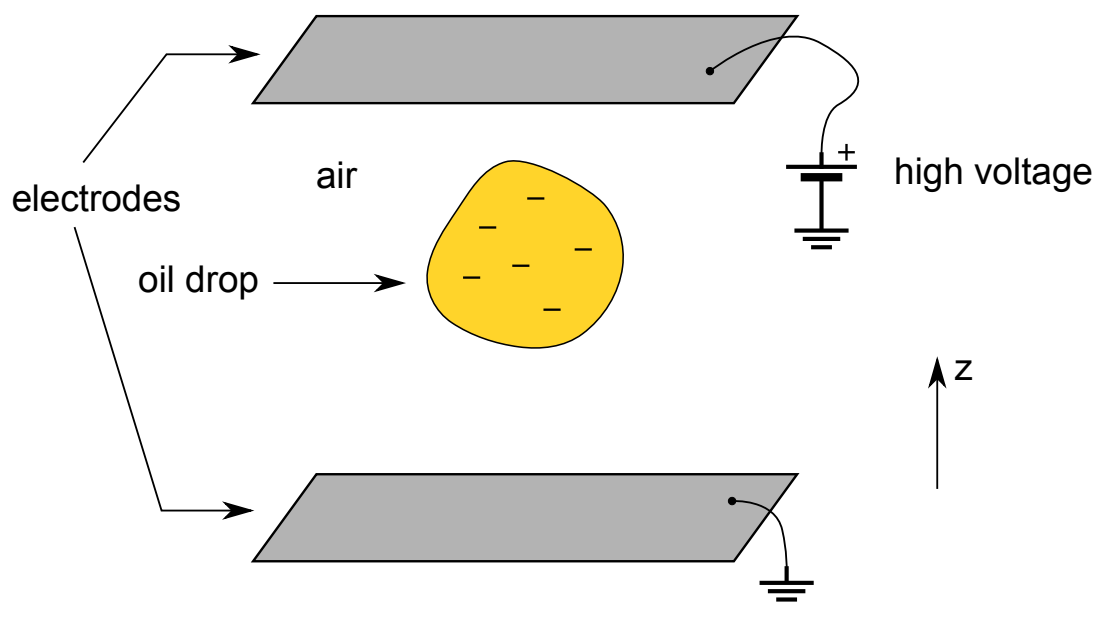

Figure 2.1: A charged oil drop falling between two electrodes. An oil drop (in yellow) with five charges in excess (minus signs) is falling (see the vertical $z$-axis). The observation of its motion between two electrodes (in gray) can give access to the elementary charge $e$. This oil drop is an isolated system: its charge can only vary by amounts $e$.

is quantized is found in the following lines. The connection strength can be evaluated by the conductance $G$ of the junction that connects the island to an electron reservoir. The typical time to discharge the capacitance $C$ through this junction is $t_{\mathrm{RC}}=(1 / G) C$. If the associated energy uncertainty $\Delta E \sim h / t_{\mathrm{RC}}$ becomes comparable to $E_{C}$ (the typical energy cost to increment the charge on the island by one), then the charge state energy is ill-defined. For a well-defined island charge, one needs $\Delta E \sim h / t_{\mathrm{RC}} \ll E_{C}=e^{2} /(2 C)$, where the capacitance $C$ can be simplified, and it comes:

$$
G \ll G_{K} \triangleq e^{2} / h
$$

Charge quantization is therefore destroyed by two types of fluctuations: (i) thermal fluctuation and (ii) quantum fluctuations.

\section{Single electron transistor (SET)}

The SET is a simple single-electron nano-device that consists of an island weakly connected to a circuit through two tunnel junctions (see Fig. 2.2 a). As in a usual transistor, the conductance $G_{\text {SET }}$ through the device can be modulated thanks to a voltage gate $V_{g}$. It has been extensively studied since its first realization in 1987 by Fulton and Dolan [42]. All the experiments we discuss in this section are based on a SET-like geometry. 

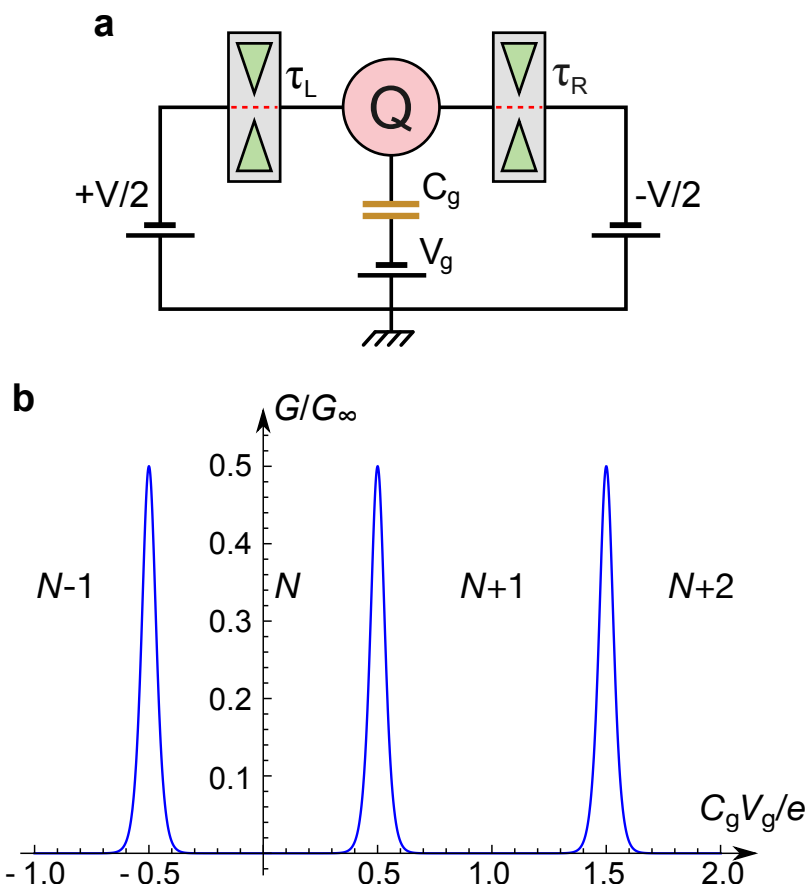

Figure 2.2: Single electron transistor and Coulomb blockade oscillations. a, Schematics of a SET. The device is d.c. biased with a voltage $V$. The excess charge $Q$ on the island (in red) is quantized at low energy for weak transmissions $\tau_{L, R} \ll 1$ of the electronic channel (dotted red lines). The charge state of the island can be tuned thanks to the voltage $V_{g}$ applied on a gate coupled capacitively $\left(C_{g}\right)$. b, Theory predicts $e$-periodic oscillations of the conductance versus $C_{g} V_{g}$. This plot is made in the linear regime of small voltage bias, $T=10 \mathrm{mK}$, and $E_{C}=300 \mathrm{mK}$ (for details, see Fig. C.3. The number $N$ of excess electrons on the island changes by one at each peak. Instead of $V_{g}$, we will rather use $N_{g} \triangleq C_{g} V_{g} / e$, the continuous control variable that tune the average number $N$ of electron on the island ( $N$ is an integer when $\left.\tau_{L, R} \ll 1\right)$.

Appendix C explains the physics of the SET. In particular, a quantitative description will be given, based on the perturbation theory (also known as the 'orthodox theory') that holds for $G \ll G_{K}$. This theory predicts 'Coulomb blockade oscillations' (see Fig. 2.2b) of the conductance versus a plunger gate voltage $V_{g}$ that are characteristic of charge quantization (the number of electron localized on the island is incremented by one after each conductance peak).

The charge state of the island in SET can therefore be controlled at the single electron level. This offers possibilities for practical applications. For instance SETs can be used as sensitive electrometers (see [38, 8] where a SET is used to probe the charge on a nearby island). Coulomb blockade in single-electron devices can also be used to perform primary thermometry (see [19] or Appendix D] 
One should increase the conductance $G$ of the connection between the island and the circuit to observe a destruction of charge quantization of the island of a SET. The intrinsic conductance of a junction can be increased by roughly two means: either one increases the conductance of a single electronic channel or one uses a wider tunnel junction (many tunnel channels in parallel, but none close to perfect transmission). Joyez and co-workers [56] have explored the second method with a metallic ${ }^{2}$ island. They observed that the charge on the island can be quantized even when the conductance of many tunnel channels in parallel exceeds the quantum of conductance $G_{K}$. In this thesis, we focus on the first method, as we will see that even a single channel at perfect transmission completely destroys the charge quantization of a metallic island.

\subsubsection{Coulomb blockade at almost perfect transmission}

The question of Coulomb blockade close to perfect transmission has been addressed in some of the first implementations of SETs in the early nineties. However the criterion to completely destroy the quantization has been established in 1995 [78]. This subsection is divided into three parts: (i) we will discuss two experiments with contradictory conclusions on the complete destruction of charge quantization in the limit of a ballistic connection between the island and the circuit; (ii) we will present the theory that explain the two conclusions depending on whether coherent effects are considered or not (iii) we will show a recent experiment in presence of coherent effects where mesoscopic conductance oscillations are clearly observed beyond the ballistic limit.

\section{Controversy on the absence of charge quantization in the limit of a ballistic connection between the island and the circuit}

First of all, let me clarify that an electronic channel reaches the 'ballistic' limit as soon as there is no backscattering of electrons. In general, the conductance of such a channel is $G=G_{K}$, the quantum of conductance. At zero magnetic field, the conductance is quantized in units of $2 \times G_{K}$ : because of spin degeneracy, pairs of two identical channels participate to transport. In presence of fractional quantum Hall effect, the ballistic limit is reached for a conductance $G=v G_{K}$, where the filling factor e.g. $v=1 / 3$ can be lower than one. In other words, the criterion is not about the conductance itself, but rather about the transmission $\tau$ of the electronic channel defined as $\tau \triangleq G / G_{\text {ballistic }}$ where $G_{\text {ballistic }}$ is the conductance in the ballistic limit.

${ }^{2}$ The metallic character of the island is of great importance as it will be explained below. 
Kouwenhoven and co-workers studied charge quantization for different magnetic field when approaching the ballistic connection limit [66, 119]. Their sample can be represented by the SET shown in Fig. $2.2 \mathrm{a}$, but with a non-metallic island. They have observed that Coulomb oscillations disappear as soon as the transmission of a channel becomes ballistic in the three regimes (at zero magnetic field, under a strong magnetic field to lift the spin degeneracy and with very strong magnetic field to reach FQHE with $v=1 / 3$ ). They have also observed that the periodicity of the oscillations is independent of the magnetic field over the full range $(10 \%$ of variation over $12 \mathrm{~T})$. This period is directly related to the geometrical capacitance $C_{g}$ (which does not change despite the compressible regions that appear due to the QHE [25]).

In contrast, Pasquier and co-workers have measured the same kind of sample, but they have observed Coulomb oscillations at zero magnetic field even beyond the ballistic limit (up to $\tau_{L}+\tau_{R} \approx 3 \times\left(2 \times G_{K}\right) /\left(2 G_{K}\right)$, where $\tau_{L}$ and $\tau_{R}$ are the transmission through the left and right QPC respectively) [100].

The reason why these two experiment have contradictory conclusions might be explained by the theory of mesoscopic Coulomb blockade [5].

\section{Coulomb blockade theory at the ballistic limit in presence or in absence of mesoscopic coherent effects}

Matveev has proposed a theory [78] in order to explain the destruction of charge quantization observed in [119] when the transmission $\tau$ of a single channel through the QPC becomes perfect $\tau \longrightarrow 1$. He modeled the channel as a 1D conductor and used the bosonization technique to demonstrate that the Hamiltonian does not depend on the voltage gate $V_{g}$ in the $\tau=1$ ballistic limit. Note that, at this point, the Coulomb blockade problem is nonperturbative in the charging energy $E_{C}$ and an exact treatment is needed [5]. At zero temperature, quantum fluctuations of the charge are known to appear when progressively connecting the island to a circuit [48] (see the dashed line in Fig. 2.3). Near the ballistic limit, Matveev has computed the charge $Q$ on the island averaged on the quantum fluctuations (neglecting the thermal fluctuation) [78]:

$$
\langle Q\rangle=e N_{g}-\gamma e / \pi \times|r| \sin 2 \pi N_{g}
$$

in the presence of a single (spinless) channel of small reflection coefficient amplitude $r \triangleq \sqrt{1-\tau} \ll 1$, and where the rescaled gate voltage $N_{g}$ was defined in Fig. 2.2 b. This expression shows quantitatively how the degree of charge quantization progressively reduces as $r \longrightarrow 0$ (see Fig. 2.3 for an illustration).

In his derivation, Matveev assumed that the mean energy level spacing $\delta E$ in the island was negligible (compared to $k_{B} T$ ). In other words, he assumed that 


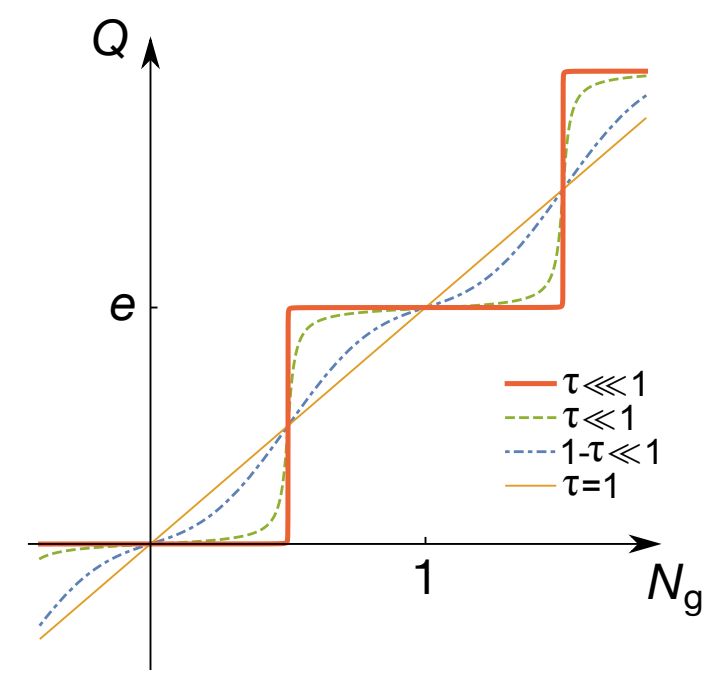

Figure 2.3: Destruction of charge quantization by quantum fluctuations at zero temperature (reproduced from [78]). The average charge $Q$ is plotted versus the gate voltage $N_{g} \triangleq C_{g} V_{g} / e$ at zero temperature for several reflection amplitudes $r \triangleq \sqrt{1-\tau}$. At $\tau \lll 1$, the island is almost isolated and $Q$ is fully quantized (solid thick line). With a small finite transmission $\tau \ll 1$, quantum fluctuation smear the steps (dashed line). The weak backscattering regime $\sqrt{1-\tau} \ll 1$ is described by Eq. 2.3) (dash-dotted line). At perfect transmission $\tau=1$, no modulation persists (thin line).

the density of state in the island was continuous. However, this is not exactly the case in the two experiments we mentioned above. A comparison of several sample characteristics is given in Table 2.1.

Aleiner and Glazman [5] have considered a finite level spacing $\delta E$ and they have found that coherence effects lead to a persistence of charge quantization even in the ballistic limit $\tau=1$ ! Indeed, the walls of the dot can reflect back the electrons into the channel (see Fig. 2.4). This elastic process depends on the path of the electron in dot. As for weak localization, interferences of electrons can modulate conductance through the dot [8]. This coherent effect leads to oscillations of the differential capacitance $C_{\text {diff }} \triangleq \mathrm{d}\langle Q\rangle / \mathrm{d} V_{g}$ that have the same period as the 'usual' Coulomb oscillations and an amplitude of $\sqrt{\delta E / E_{C}}$ for spinless electrons and $\left(\delta E / E_{C}\right) \ln ^{2}\left(\delta E / E_{C}\right)$ for spin-half electrons.

These oscillations that may occur in quantum dots (islands with a discrete density of state) even when they are open (connected to a lead with a channel of ballistic transmission $\tau=1$ ) are called 'mesoscopic Coulomb blockade' oscillations. As they require phase coherence, they highly depend on temperature and magnetic field. 


\begin{tabular}{|c|c|c|c|c|}
\hline First author & $T_{\text {base }}$ & $\delta E / k_{B}$ & $E_{C} / k_{B}$ & reference \\
\hline \hline Kouwenhoven & $10 \mathrm{mK}$ & $120 \mathrm{mK}$ & $7.0 \mathrm{~K}$ & {$[[66]$} \\
\hline Pasquier & $60 \mathrm{mK}$ & $85 \mathrm{mK}$ & $2.2 \mathrm{~K}$ & {$[[100]$} \\
\hline Crouch & $75 \mathrm{mK}$ & $400 \mathrm{mK}$ & $2.7 \mathrm{~K}$ & {$[35]$} \\
\hline Cronenwett & $100 \mathrm{mK}$ & $163 \mathrm{mK}$ & $3.3 \mathrm{~K}$ & {$[34]$} \\
\hline Amasha & $13 \mathrm{mK}$ & $31 \mathrm{mK}$ & $1.3 \mathrm{~K}$ & {$[[8]$} \\
\hline Jezouin & $17 \mathrm{mK}$ & $0.2 \mu \mathrm{K}$ & $0.3 \mathrm{~K}$ & {$[55$} \\
\hline
\end{tabular}

Table 2.1: Comparison of several experiment characteristics. Our experiment (last line) based on a metallic island with a negligible $\delta E$ is given for comparison. Mesoscopic Coulomb blockade have been observed in all the listed experiments except in Kouwenhoven's one and in ours.

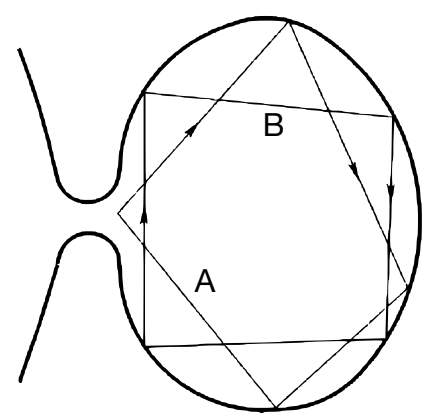

Figure 2.4: Mesoscopic effect in a quantum dot (reproduced from [5]). In some experiments (not in ours), electrons can be coherently reflected back to the electronic reservoir (on the left) leading to a modulation of the differential capacitance that depends on the trajectory (e.g. A or B).

\section{Mesoscopic Coulomb blockade observations}

In principle, mesoscopic Coulomb blockade should have been observed in the experiment of Kouwenhoven and co-workers [66]. Indeed, in the SET regime, they have observed that the conductance through the two tunnel junctions $\left(\tau_{1}, \tau_{2} \ll 1\right)$ in series was much larger $\left(G_{\mathrm{SET}} \approx 0.8 G_{K}\right)$ than the classical serial conductance. At low temperature $T$ compared to the level spacing $k_{B} T \ll \delta E$, the system enters the quantum regime [65] where coherent effects are relevant. According to Aleiner and Glazman [5], a possible reason why Kouwenhoven and co-workers have not observed mesoscopic Coulomb blockade is that the shape of their quantum dot might not be suitable to host the chaotic paths required by the theory.

After the experiment of Pasquier and co-workers [100], other teams have observed a persistence of quantization in the limit of a ballistic channel connection [35, 34, 8]. They have measured a temperature dependence on these Coulomb oscillations. In [34] and [8], a strong dependence on the magnetic field have been 


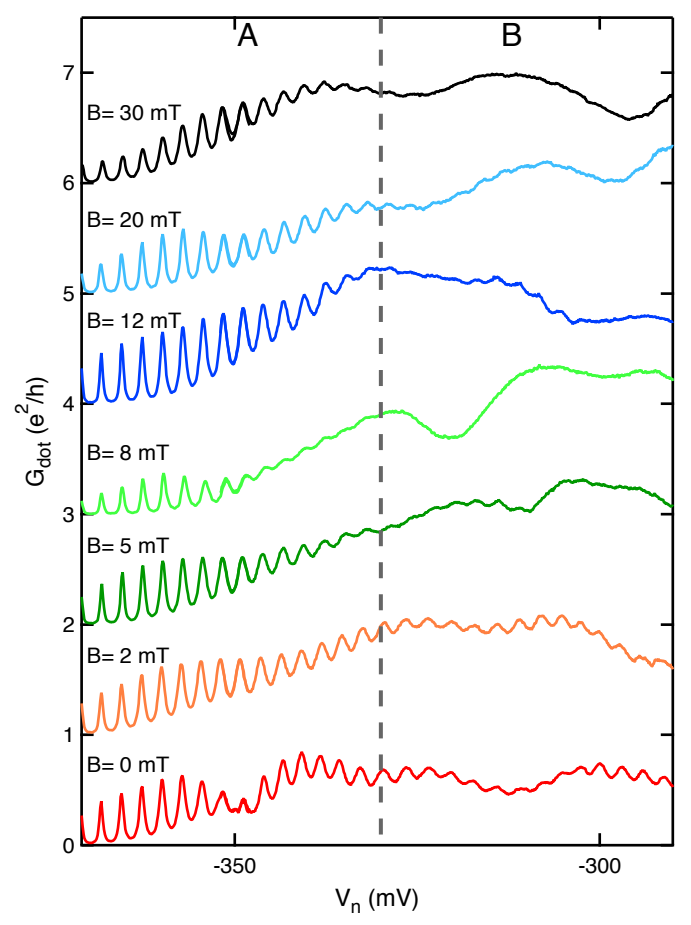

Figure 2.5: Mesoscopic Coulomb blockade observation by Amasha and coworkers $[\mathbf{8}]$. For several magnetic field $B$, the conductance $G_{\mathrm{dot}}$ is plotted versus a voltage gate $V_{n}$ that controls the opening of the dot. In region A, the dot is partially connected to the circuit; whereas is region $\mathrm{B}$, the dot is open (an electronic channel is fully transmitted). Conductance oscillations are observed in region B, they are strongly affected by a finite magnetic field (in contrast to the 'usual' Coulomb blockade oscillations observed in region A).

observed (see Fig. 2.5). These two dependences are characteristic of mesoscopic phenomena as they involve the quantum phase of the electrons.

In our implementation, we use a metallic island where $\delta E$ is six orders of magnitude smaller than $E_{C}$ and thus avoid any mesoscopic Coulomb blockade. That is why our sample specifically probes (usual) Coulomb blockade without any coherent effect 'artifact'. Theoretical predictions on how charge quantization vanishes in this case is presented in the following section.

\subsection{Theoretical predictions on the charge quantiza- tion of a metallic island without coherent effect}

In addition to avoid mesoscopic Coulomb blockade, considering a metallic island without coherent effect has the advantage to simplify the problem for the theorists. 
In this section we will give some quantitative prediction on the conductance $G_{\text {SET }}$ at zero voltage bias $V=0$ (see Fig. 2.2 a) in the quantum regime (low temperature $\left.k_{B} T \ll E_{C}\right)$ in two limit cases: the asymmetric case $\tau_{L} \ll 1,1-\tau_{R} \ll 1$ and the symmetric case $1-\tau_{L, R} \ll 1$.

We will first explain how both the conductance $G_{\text {SET }}$ and the differential capacitance $C_{\text {diff }}$ characterize the charge quantization. Then we will present the predictions in the two limit cases (asymmetric and symmetric). For each case, we will separate the low temperature quantum regime and the high temperature regime. The latter regime where many charge states are thermally activated has been treated by our collaborators I.P. Levkivskyi, E. Idrisov, E.V. Sukhorukov and L.I. Glazman to explain our experimental data (shown in the next section). We will see that all the predictions scales with reflection coefficient amplitude $\sqrt{1-\tau}$.

\subsubsection{Transport versus thermodynamic properties}

The quantized aspect of the charge on the island can be probed either through transport or thermodynamic properties. The former consists in the measurement of the conductance $G_{\text {SET }}$ of the elements $\mathrm{QPC}_{L}$-island-QPC ${ }_{R}$ in series. The latter is based on the differential capacitance $C_{\text {diff }}$ to the island.

We have not access to the differential capacitance $C_{\text {diff }}$ with our sample because it requires an additional electrometer (for example, see [8] where two quantum dots have been used: a big one as an island, and a small one as an electrometer). This quantity $C_{\text {diff }} \triangleq \mathrm{d}\langle Q\rangle / \mathrm{d} V_{g}$ is directly related to the average charge $\langle Q\rangle$ on the island. Note that a single lead can be used when studying $C_{\text {diff }}$ since the current through the device is not considered when dealing with thermodynamic properties.

The $e$-periodic oscillations of the conductance $G_{\mathrm{SET}}$ versus the plunger gate $V_{g}$ that we measured are also a clear signature of charge quantization. However, in contrast with $C_{\text {diff }}$, the measure of $G_{\mathrm{SET}}$ does not give a direct access to the average excess charge $Q$ on the island. Nonetheless, we will show that both $G_{\mathrm{SET}}$ and $C_{\text {diff }}$ have similar dependences on the relevant parameters. Moreover, the conductance is important in the applications of single-electronics [37].

\subsubsection{Asymmetric case $\tau_{L} \ll 1,1-\tau_{R} \ll 1$}

Low temperatures $k_{B} T \ll E_{C}$

The asymmetric limit has been studied by Matveev when he showed that charge quantization vanishes when an electronic channel connects ballistically the island [79]. We can find the visibility of the oscillations of the differential capacitance $C_{\text {diff }}$ from his expression of the average charge given in Eq. (2.3): 


$$
\Delta C_{\mathrm{diff}}\left(\tau_{L} \ll 1,1-\tau_{R} \ll 1\right) \triangleq \frac{C_{\mathrm{diff}}^{\max }-C_{\mathrm{diff}}^{\min }}{C_{\mathrm{diff}}^{\max }+C_{\mathrm{diff}}^{\min }}=2 \gamma \sqrt{1-\tau_{R}}
$$

where $\gamma \approx \exp (0.5772)$ is the exponential of Euler's constant. The quantitative prediction for the serial conductance through the two asymmetric junctions is given by (Equation 34 in [80]):

$$
G_{\mathrm{SET}}\left(\tau_{L} \ll 1,1-\tau_{R} \ll 1, N_{g}\right)=\tau_{L} G_{K} \times \frac{2 \pi^{4} T^{2}}{3 \gamma^{2} E_{C}^{2}}\left[1-2 \gamma \xi \sqrt{1-\tau_{R}} \cos \left(2 \pi N_{g}\right)\right]
$$

where $\xi \approx 1.59$ is a numerical coefficient. This expression is valid only for $1-\tau_{R} \ll 1$ (as one can see that the minimal value of the conductance in Eq. (2.5) is strikingly incorrect, negative, already for $\left.\tau_{R} \leq 1-1 /(2 \gamma \xi)^{2} \approx 0.97\right)$. The visibility of the Coulomb blockade oscillations of the conductance comes directly from the quantitative expression of $G_{\mathrm{SET}}$ :

$$
\Delta Q\left(\tau_{L} \ll 1,1-\tau_{R} \ll 1\right) \triangleq \frac{G_{\mathrm{SET}}^{\max }-G_{\mathrm{SET}}^{\min }}{G_{\mathrm{SET}}^{\max }+G_{\mathrm{SET}}^{\min }}=2 \gamma \xi \sqrt{1-\tau_{R}}
$$

We see that in the case of asymmetric junctions, at low temperature $T \ll E_{C}$, the visibility on the oscillations of the differential capacitance $C_{\text {diff }}$ is proportional to the visibility on the conductance oscillations $G_{\mathrm{SET}}: \Delta Q=\xi \Delta C_{\text {diff }}$, as explicitly pointed out by Yi and Kane in [134].

\section{High temperature limit $k_{B} T \gg E_{C} / \pi^{2}$}

At high temperatures $k_{B} T \gg E_{C}$, several charge states are populated. This situation has not been considered by Matveev in [78]. However, his reasoning can be extended to this case by averaging over the Gibbs distribution of fluctuations (see the Methods section of our article [55]). Our collaborators have then derived the expression of the differential capacitance at high temperature, in the asymmetric limit:

$C_{\text {diff }}\left(\tau_{L}=0,1-\tau_{R} \ll 1, N_{g}\right) \approx \frac{e}{\Delta V_{g}}-4 \frac{e}{\Delta V_{g}} \frac{\pi^{2} k_{B} T}{E_{C}} \exp \left(-\frac{\pi^{2} k_{B} T}{E_{C}}\right) \sqrt{1-\tau_{R}} \cos \left(2 \pi N_{g}\right)$

This expression has been derived using also [90], which is a general theory not assuming $1-\tau_{R} \ll 1$ at large temperatures $k_{B} T \gg E_{C} / \pi^{2}$. This expression matches the prediction for an almost isolated island $\left(\tau_{L, R} \ll 1\right)$, just by taking the limit $\sqrt{1-\tau_{R}} \longrightarrow 1$. The visibility on these $C_{\text {diff }}$ oscillations is 


$$
\Delta C_{\mathrm{diff}}\left(\tau_{L}=0,1-\tau_{R} \ll 1\right) \approx 4 \frac{\pi^{2} k_{B} T}{E_{C}} \exp \left(-\frac{\pi^{2} k_{B} T}{E_{C}}\right) \sqrt{1-\tau_{R}}
$$

it is exponentially reduced at high temperatures and it depends as $\sqrt{1-\tau_{R}}$ with the transmission. The conductance in the asymmetric and high temperature regime can be obtained starting from Eq. 2.5] in [43] or by using a specific method (see the Methods section of our article [55] and the unpublished ref. [73] of our collaborators). We get a visibility on $G_{\mathrm{SET}}$ oscillations as:

$$
\Delta Q\left(\tau_{L} \ll 1,1-\tau_{R} \ll 1\right) \approx \exp \left(-\frac{\pi^{2} k_{B} T}{E_{C}}\right) \sqrt{1-\tau_{R}}
$$

The same dependence on the transmission $\tau_{R}$ is predicted for the visibility of both the $C_{\text {diff }}$ and $G_{\text {SET }}$ oscillations. And an exponential reduction with the temperature is also expected at high temperature for the conductance oscillations.

\subsubsection{Symmetric limit $1-\tau_{L, R} \ll 1$}

Low temperatures $k_{B} T \ll E_{C}$

The conductance in the strong coupling limit $1-\tau_{L, R} \ll 1$ with two channels almost perfectly transmitted has been studied by Furusaki and Matveev [43]. They have derived the quantitative expression for the serial conductance (see Equation 38 obtained in [43] and Equation A9 for the $T$-linear term which is the leading-order correction in $\left.k_{B} T / E_{C}\right)$ :

$$
\begin{aligned}
G_{\mathrm{SET}}\left(1-\tau_{L} \ll 1,1-\tau_{R} \ll 1, N_{g}\right)=G_{K} / 2 \times[1- \\
\\
\quad \frac{\gamma E_{C} \Gamma_{+}\left(\tau_{L}, \tau_{R}, N_{g}\right)}{\pi^{3} k_{B} T} \psi^{\prime}\left(1 / 2+\frac{\gamma E_{C} \Gamma_{+}\left(\tau_{L}, \tau_{R}, N_{g}\right)}{\pi^{3} k_{B} T}\right) \\
\left.\quad-\frac{\pi^{3} \gamma k_{B} T}{16 E_{C}} \times \Gamma_{-}\left(\tau_{L}, \tau_{R}, N_{g}\right)\right]
\end{aligned}
$$

where we have replaced the original integral by the digamma function ${ }^{3}$ and where

\footnotetext{
${ }^{3}$ We have verified numerically that $\frac{1}{4 T} \int_{-\infty}^{\infty} \mathrm{d} E \frac{1}{\cosh ^{2}\left(E /\left(2 k_{B} T\right)\right)} \frac{\Gamma_{+}^{2}\left(\tau_{L}, \tau_{R}, N_{g}\right)}{E^{2}+\Gamma_{+}^{2}\left(\tau_{L}, \tau_{R}, N_{g}\right)}=$ $\frac{\Gamma_{+}\left(\tau_{L}, \tau_{R}, N_{g}\right)}{2 \pi k_{B} T} \psi^{\prime}\left(1 / 2+\frac{\Gamma_{+}\left(\tau_{L}, \tau_{R}, N_{g}\right)}{2 \pi k_{B} T}\right)$.
} 


$$
\Gamma_{ \pm}\left(\tau_{L}, \tau_{R}, N_{g}\right)=\left(1-\tau_{L}\right)+\left(1-\tau_{R}\right) \pm 2 \sqrt{\left(1-\tau_{L}\right)\left(1-\tau_{R}\right)} \cos \left(2 \pi N_{g}\right)
$$

The visibility of the Coulomb blockade oscillations at low temperature $k_{B} T / E_{C} \ll$ 1 and in the strong coupling reads:

$$
\Delta Q\left(1-\tau_{L, R} \ll k_{B} T / E_{C} \ll 1\right)=\frac{\gamma E_{C}}{\pi k_{B} T} \sqrt{\left(1-\tau_{L}\right)\left(1-\tau_{R}\right)}
$$

The differential capacitance has been evaluated at low temperature in the strong couping regime when first $\tau_{L} \longrightarrow 1$ and then $\tau_{R} \longrightarrow 1$ (Equation 41 in [71]):

$$
C_{\text {diff }}\left(1-\tau_{L} \ll 1-\tau_{R} \ll 1, N_{g}\right)=\frac{e}{\Delta V_{g}}-4 \gamma \frac{e}{\Delta V_{g}} \ln \left(1-\tau_{L}\right) \sqrt{\left(1-\tau_{L}\right)\left(1-\tau_{R}\right)} \cos \left(2 \pi N_{g}\right)
$$

It yields a visibility on $C_{\mathrm{diff}}$ oscillations as:

$$
\Delta C_{\mathrm{diff}}\left(1-\tau_{L} \ll 1-\tau_{R} \ll 1\right)=4 \gamma \ln \left(1-\tau_{L}\right) \sqrt{\left(1-\tau_{L}\right)\left(1-\tau_{R}\right)}
$$

In this regime also, a dependence in $\sqrt{\left(1-\tau_{L}\right)\left(1-\tau_{R}\right)}$ is found for the visibility on both the $G_{\mathrm{SET}}$ and $C_{\text {diff }}$ oscillations. However, a 'log' appears in the expressions of $\Delta C_{\text {diff }}$.

Note that for exactly symmetric transmissions $\tau \triangleq \tau_{L}=\tau_{R}$, the differential capacitance diverges at the degeneracy point $N_{g}=0$ (Equation 49 in [78] or Equation 32 in [71]):

$C_{\text {diff }}\left(1-\tau \ll 1, N_{g}\right)=\frac{e}{\Delta V_{g}}-4 \gamma \frac{e}{\Delta V_{g}} \ln \left((1-\tau) \sin \left(\pi N_{g}\right)\right) \times(1-\tau) \cos \left(2 \pi N_{g}\right)$

This divergence is due to the two-channel Kondo effect, which will be the topic of Chapter 3. It disappears as soon as an asymmetry $\tau_{L} \neq \tau_{R}$ is introduced [71]. This critical phenomenon will be discussed in Chapter 4 .

\section{High temperature regime $k_{B} T \gg E_{C} / \pi^{2}$}

As for the asymmetric case, our collaborators have computed the conductance in the high temperature regime. They have found an expression that gives a visibility of:

$$
\Delta Q\left(1-\tau_{L, R} \ll 1\right) \approx \exp \left(-\frac{\pi^{2} k_{B} T}{E_{C}}\right) \sqrt{1-\tau_{L}} \sqrt{1-\tau_{R}}
$$


In this strong coupling regime also, we see that Coulomb blockade oscillations on the conductance are exponentially reduced at high temperatures. The same behavior is expected for the visibility of the differential capacitance oscillation (this quantity has not been computed by our collaborators, but no interplay between the channels will occur out of the quantum regime $k_{B} T \ll E_{C}$ ).

\subsubsection{Universality in the high temperature regime}

The predictions beyond the tunnel regime require advanced theoretical methods. In particular, as we will see in Chapter 3, a reason is that the Coulomb blockade with a metallic island and a few electronic channels can exhibit Kondo effect at low temperature, depending on the gate voltage $V_{g}$.

The predictions we have presented above are based on the bosonization technique. Another method consists in using the instanton solution of Korshunov [63]. These instantons have been used in [110] to predict an exponential suppression of the charge quantization with the temperature. This technique has been used also by Nazarov to propose a general solution of the Coulomb blockade problem beyond the tunnel limit [90].

His solution is presented for thermodynamic quantities (such as $C_{\text {diff }}$ ) only. The charging energy $E_{C}$ is shown to have effective modulations due to the gate voltage $V_{g}$ that scale as $\tilde{E}_{C} \propto E_{C} \prod_{j} \sqrt{1-\tau_{j}}$, where the product is done on the electronic channel index $j$. This expressions suggests that the suppression of the charge quantization becomes universal when rescaled with respect to $\sqrt{1-\tau}$.

This prediction is not valid at low temperature ${ }^{4}$ as it does not predict the $\log$ divergence on $C_{\mathrm{diff}}$ for symmetric transmissions near the ballistic limit (see Eq. (2.12) and Section 2.2.3).

\subsection{Controlling charge quantization with quantum fluctuations}

We have just published our experimental data on the charge quantization of a metallic island on August 4th 2016 in Nature journal (doi:10.1038/nature19072). The text of the article is reprinted in this section. An additional figure (Fig. 2.6) is given (as in the online version of the paper), where we show a quantitative comparison of our experimental data with the predictions Eqs. 2.5) and (2.9).

The sample we use is described and characterized in details in Appendix B

\footnotetext{
${ }^{4}$ Probably because of the large fluctuations of the phase at low temperature and $\sqrt{1-\tau} \ll 1$ that makes the instanton technique less accurate (private discussion with our collaborators).
} 


\section{Controlling charge quantization with quantum fluctuations}

S. Jezouin ${ }^{1 *}$, Z. Iftikhar ${ }^{1}$, A. Anthore ${ }^{1}$, F. D. Parmentier ${ }^{1}$, U. Gennser $^{1}$, A. Cavanna ${ }^{1}$, A. Ouerghi ${ }^{1}$, I. P. Levkivskyi ${ }^{2}$, E. Idrisov $^{3}$, E. V. Sukhorukov ${ }^{3}$, L. I. Glazman ${ }^{4} \&$ F. Pierre ${ }^{1}$

In 1909, Millikan showed that the charge of electrically isolated systems is quantized in units of the elementary electron charge $e$. Today, the persistence of charge quantization in small, weakly connected conductors allows for circuits in which single electrons are manipulated, with applications in, for example, metrology, detectors and thermometry ${ }^{1-5}$. However, as the connection strength is increased, the discreteness of charge is progressively reduced by quantum fluctuations. Here we report the full quantum control and characterization of charge quantization. By using semiconductor-based tunable elemental conduction channels to connect a micrometre-scale metallic island to a circuit, we explore the complete evolution of charge quantization while scanning the entire range of connection strengths, from a very weak (tunnel) to a perfect (ballistic) contact. We observe, when approaching the ballistic limit, that charge quantization is destroyed by quantum fluctuations, and scales as the square root of the residual probability for an electron to be reflected across the quantum channel; this scaling also applies beyond the different regimes of connection strength currently accessible to theor ${ }^{6-8}$. At increased temperatures, the thermal fluctuations result in an exponential suppression of charge quantization and in a universal square-root scaling, valid for all connection strengths, in agreement with expectations ${ }^{8}$. Besides being pertinent for the improvement of single-electron circuits and their applications, and for the metal-semiconductor hybrids relevant to topological quantum computing ${ }^{9}$, knowledge of the quantum laws of electricity will be essential for the quantum engineering of future nanoelectronic devices.

Some of the most fundamental theoretical predictions have so far eluded experimental confirmation. Charging effects are generally found to diminish as the conductances of the contacts are increased ${ }^{10-18}$; however, although some measurements support the fundamental prediction ${ }^{6-8}$ that charge quantization vanishes in the presence of one ballistic channel ${ }^{10-12,17}$, others conclude the opposite ${ }^{18-23}$. Unsurprisingly, the scaling behaviour predicted for the reduction of charge quantization ${ }^{6-8}$ has also remained elusive, until now, despite several attempts ${ }^{16,17}$.

A plausible explanation of the varying results regarding the charge quantization criteria is that, in the previously investigated devices, the quantum channels and the conductor were not completely distinct circuit elements. With a small island, in which the density of states is discrete, the non-local electronic wave functions merge the connected channels and the island into a complex quantum conductor, where Coulomb interactions may play a non-trivial role. As a result, charging effects can develop even if one of the conduction channels taken separately is perfectly ballistic. This phenomenon is called mesoscopic Coulomb blockade ${ }^{18,22,24}$.

Investigating charge quantization at the most elemental singlechannel level therefore requires tunable conduction channels linked to a conductor with a negligible electronic level spacing. Although this can be realized by making the island larger, its size must remain small enough to preserve charge quantization. Indeed, thermal fluctuations average out charge quantization unless the charging energy associated with the addition of one electron in the island $-E_{\mathrm{C}}=e^{2} / 2 C$, where the geometrical capacitance of the island $C$ increases with size-is larger than the thermal energy $k_{\mathrm{B}} T$, with $k_{\mathrm{B}}$ the Boltzmann constant and $T$ the temperature ${ }^{1,2}$.

We have solved these conflicting requirements with the hybrid metal-semiconductor single-electron transistor (SET) shown in Fig. 1a, implementing the schematic circuit of Fig. 1b: a central metallic island with a continuous density of states (coloured red in Fig. 1a, b) is connected to large electrodes (represented by white disks) through two $\mathrm{Ga}(\mathrm{Al})$ As quantum point contacts $\left(\mathrm{QPC}_{\mathrm{L}, \mathrm{R}}\right)$ that emulate single-channel quantum conductors over the entire range of coupling strengths.

The metallic island, which is made of a metallic AuGeNi alloy, has a negligible electronic level spacing $\delta \approx k_{\mathrm{B}} \times 0.2 \mu \mathrm{K}$, five orders of magnitude smaller than the base electronic temperature $T \approx 17 \mathrm{mK}$. It is galvanically connected, by thermal annealing, to a 105 -nm-deep, $\mathrm{Ga}(\mathrm{Al}) \mathrm{As}$, high-mobility two-dimensional electron gas (2DEG; darker grey areas delimited by bright lines in Fig. 1a). Achieving an almost perfectly transparent metal-2DEG electrical contact is crucial to reach the ballistic channel limit. Remarkably, the reflection probability of electrons at the interface is below $0.05 \%$.

The QPCs are located in the 2DEG and tuned by field effect with the voltage applied to capacitively coupled metallic split gates (coloured green in Fig. 1a; the top-right split gates that are coloured yellow are negatively biased to remove the 2DEG underneath). Besides tuning, the precise characterization of each QPC, independently, is necessary for the quantitative exploration of charge quantization versus connection strength. However, in the SET configuration, the QPC conductances are interconnected and renormalized by Coulomb blockade. Moreover, only their series combination is accessible. To completely characterize $\mathrm{QPC}_{\mathrm{L}, \mathrm{R}}$, we implemented with adjacent gates (coloured blue in Fig. 1a) the on-chip switches shown in Fig. 1b. The measured quantities $\tau_{\mathrm{L}, \mathrm{R}} \equiv G_{\mathrm{L}, \mathrm{R}}^{\mathrm{qp}} h / e^{2}$ (with $h$ the Planck constant and $G_{\mathrm{L}, \mathrm{R}}^{\mathrm{qpc}}$ the conductances of $\mathrm{QPC} \mathrm{L}_{\mathrm{L}, \mathrm{R}}$ when switches are closed (inset of Fig. 1c)) directly give the 'intrinsic' (not renormalized by Coulomb blockade) electron transmission probabilities of the constitutive quantum channels, which fully characterize the connection strength to the metallic island. As illustrated in Fig. $1 \mathrm{c}, \tau_{\mathrm{L}(\mathrm{R})} \leq 1$ corresponds to a single (spin-polarized, see below) channel of transmission probability $\tau_{\mathrm{L}(\mathrm{R})}$ across $\mathrm{QPC}_{\mathrm{L}(\mathrm{R})}$. For $1<\tau_{\mathrm{R}} \leq 2$, there are two channels across $\mathrm{QPC}_{\mathrm{R}}$-one fully ballistic and the other with transmission probability $\tau_{\mathrm{R}}-1$. With this approach, we achieve an accuracy down to $0.1 \%$ near the ballistic limit.

The sample is immersed into a perpendicular magnetic field $B \approx 4 \mathrm{~T}$, which corresponds to the integer quantum Hall effect at filling 
a

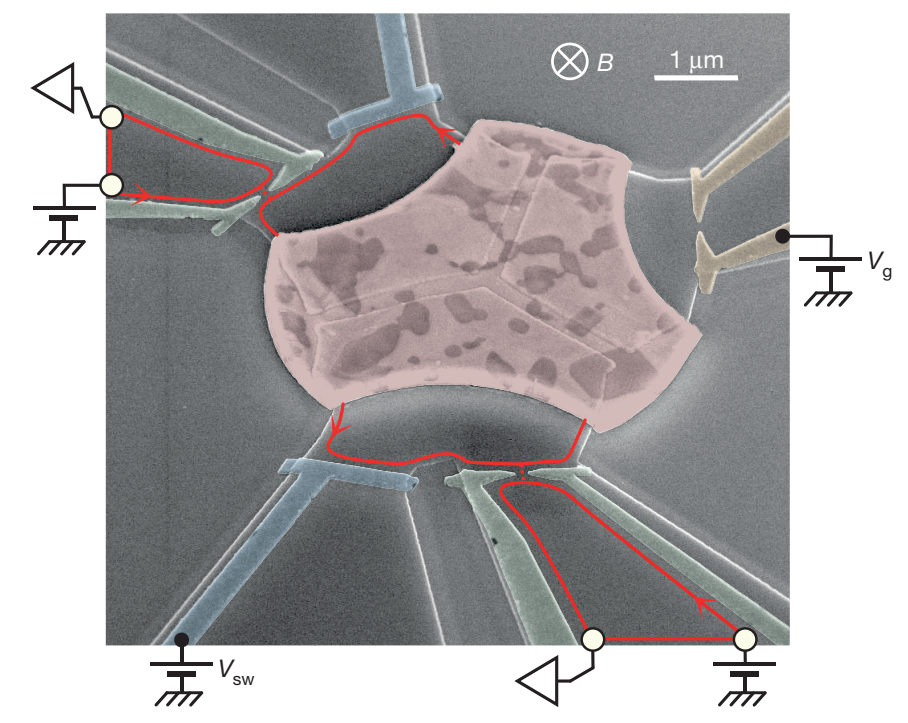

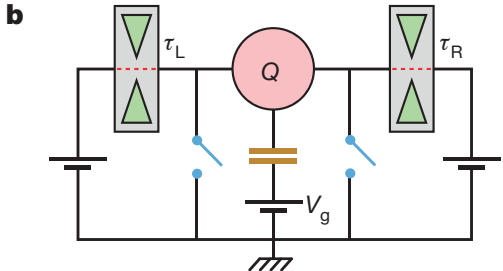

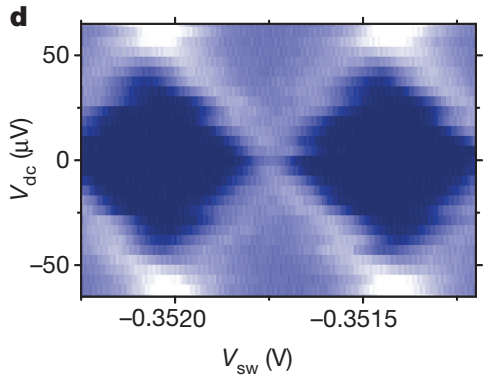

Figure 1 | Tunable quantum connection to a metallic island. a, Coloured sample micrograph. A micrometre-scale metallic island (red) is connected to large electrodes (white circles) through two quantum point contacts (QPCs, green split gates) formed in a buried two-dimensional electron gas (2DEG; darker grey delimited by bright lines). The lateral gates (blue) implement short-circuit switches as shown in $\mathbf{b}$. The yellow gates, tuned at $V_{\mathrm{g}}$ negative enough to deplete the 2DEG underneath, are capacitively coupled to the island and used to evidence charge quantization. In the applied field $B \approx 4 \mathrm{~T}$, the current propagates along two edge channels (red lines) in the direction indicated by arrows. b, Sample schematic; colours as in a; $Q$ represents the excess charge that can accumulate on the

factor $\nu=2$. In this regime, the electrical current propagates along two edge channels (shown as a single red line in Fig. 1a) in the direction indicated by arrows, which does not influence charge quantization (for a specific discussion see Methods section 'Conductance in the near-ballistic regime with strong thermal fluctuations'). The large Zeeman splitting results in the full separation between the successive openings of the two spin-polarized quantum channels across the QPCs (Fig. 1c).

Charge quantization in the central island is unequivocally evidenced by periodic oscillations of the SET differential conductance $G_{\text {SET }}$ (across $\mathrm{QPC}_{\mathrm{L}}$-island- $\mathrm{QPC}_{\mathrm{R}}$ ) when sweeping a capacitively coupled gate voltage, which develop into Coulomb diamonds with d.c. bias voltage $V_{\mathrm{dc}}$ (Fig. 1d). With both QPCs in the tunnel regime, $\tau_{\mathrm{L}, \mathrm{R}} \ll 1$, the span of the diamonds in $V_{\mathrm{dc}}$ gives the charging energy $E_{\mathrm{C}} \approx k_{\mathrm{B}} \times 0.3 \mathrm{~K}$ $(C \approx 3.1 \mathrm{fF})$.

We first probe the evolution of charge quantization with transmission probability directly from $G_{S E T}$ raw periodic modulations. Figure 2a

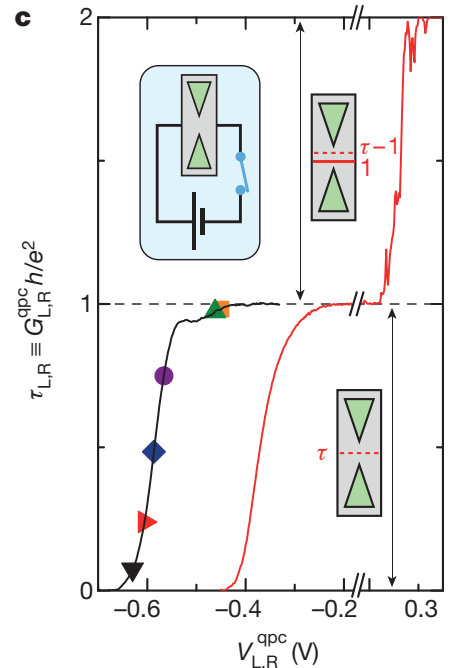

metallic island. c, The 'intrinsic' (switch closed; see inset schematic) conductance $G_{\mathrm{L}, \mathrm{R}}^{\mathrm{qpc}}$ across $\mathrm{QPC}_{\mathrm{L}, \mathrm{R}}$ (shown top-right and bottom left, respectively, in a) is shown versus split gate voltage $V_{\mathrm{L}, \mathrm{R}}^{\mathrm{qpc}}$ as black (L) and red $(\mathrm{R})$ lines. Symbols indicate the set-points of $\mathrm{QPC}, \mathrm{L}$ used thereafter. The number and transmission probabilities of electronic channels through the QPC (pair of green triangles) are schematized for $\tau_{\mathrm{R}}<1$ and $\tau_{\mathrm{R}}>1$ : a dashed (solid) red line represents a partially (perfectly) transmitted channel. d, Coulomb diamond patterns in the device conductance $G_{\text {SET }}$ (larger shown brighter, from 0 in dark blue up to $0.13 e^{2} / h$ in white) measured versus gate $\left(V_{\mathrm{sw}}\right)$ and bias $\left(V_{\mathrm{dc}}\right)$ voltages for tunnel contacts $\tau_{\mathrm{L}, \mathrm{R}} \ll 1$.

displays $G_{\mathrm{SET}}$ measured at $T \approx 17 \mathrm{mK}$ and $V_{\mathrm{dc}}=0$ while sweeping the capacitively coupled gate voltage $V_{\mathrm{g}}$ (Fig. 1a), for QPC $\mathrm{C}_{\mathrm{L}}$ fixed to $\tau_{\mathrm{L}}=0.24$ and with each panel corresponding to a different $\mathrm{QPC}_{\mathrm{R}}$ tuning $\left(\tau_{\mathrm{R}}=0.1,0.6,0.88,0.98\right.$ and 1.5 , from left to right). These raw data reveal the remarkable robustness of charge quantization to connection strength. At $\tau_{\mathrm{R}}=0.1$ and $\tau_{\mathrm{R}}=0.6$, the presence of sharp periodic peaks separated by intervals in which $G_{\mathrm{SET}} \approx 0$ signals an essentially unaltered charge quantization over the greater part of transmission probabilities. Although $G_{\mathrm{SET}}\left(\delta V_{\mathrm{g}}\right)$ progressively evolves with increasing $\tau_{\mathrm{R}}<1$ into a sinusoid with non-zero minima, relatively important modulations of fixed ( $\tau_{\mathrm{R}}$-independent $)$ period persist very close to the ballistic limit, at $\tau_{\mathrm{R}}=0.98$. In stark contrast, $G_{\mathrm{SET}}$ is independent of $V_{\mathrm{g}}$ at $\tau_{\mathrm{R}}=1.5$, confirming the predicted complete collapse of charge quantization in the presence of a fully ballistic channel. Note that $G_{S E T}$ remains reduced by Coulomb interactions, even at $\tau_{\mathrm{R}}=1.5$, as evidenced by the pronounced conductance dip at low $V_{\mathrm{dc}}$ (inset of Fig. 2b). 

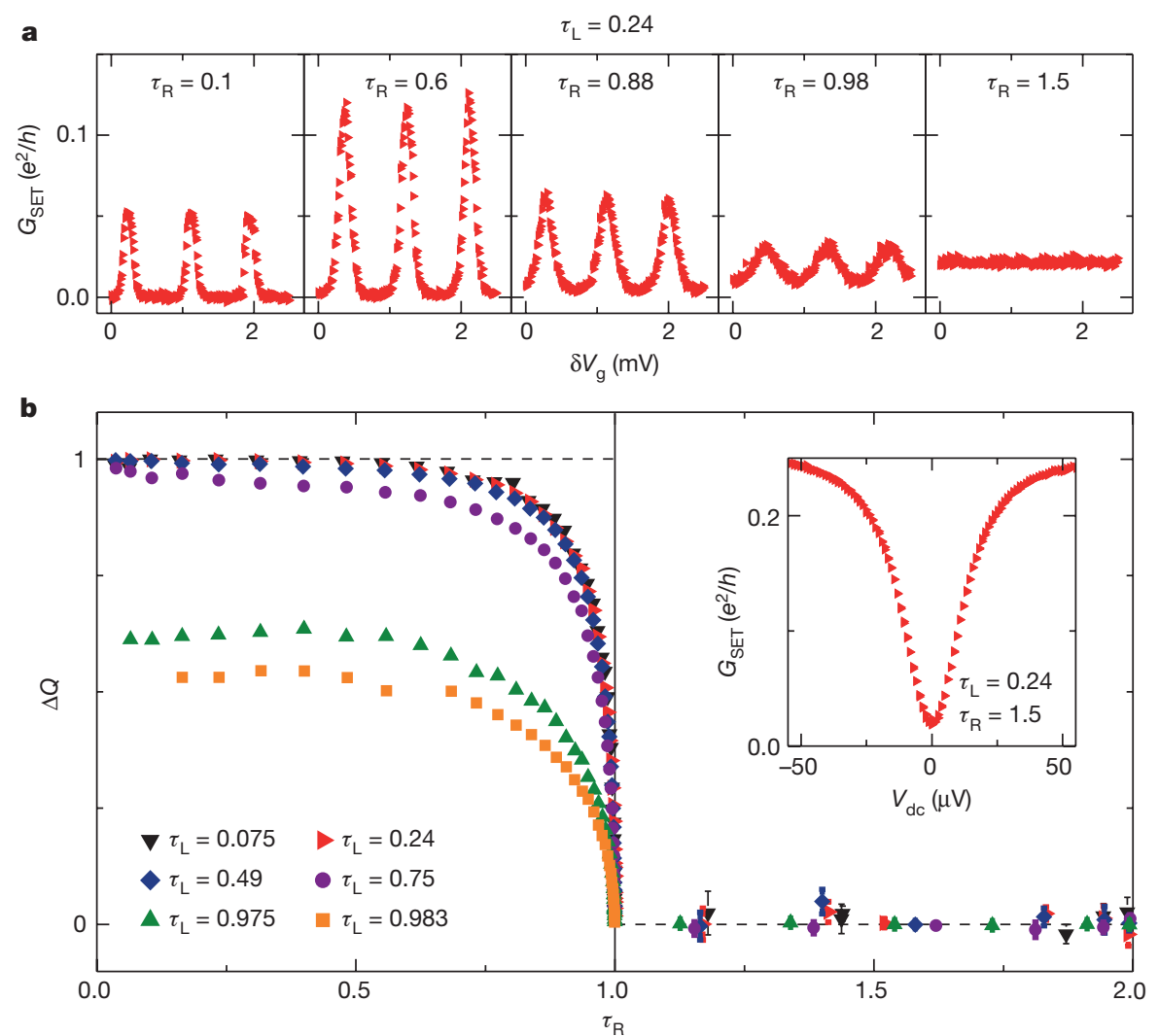

Figure 2 Charge quantization versus connection strength at $T \approx 17 \mathbf{m K}$. a, Conductance sweeps $G_{\mathrm{SET}}\left(\delta V_{\mathrm{g}}\right)$ with fixed $\tau_{\mathrm{L}}=0.24$ and varying $\tau_{\mathrm{R}}=0.1,0.6,0.88,0.98$ and 1.5 , from left to right, as indicated. b, Visibility of $G_{\mathrm{SET}}$ oscillations $\Delta Q \equiv\left(G_{\mathrm{SET}}^{\max }-G_{\mathrm{SET}}^{\min }\right) /\left(G_{\mathrm{SET}}^{\max }+G_{\mathrm{SET}}^{\min }\right)$ versus $\tau_{\mathrm{R}}$, with each set of symbols corresponding to a different $\mathrm{QPC}_{\mathrm{L}}$ set-point $\tau_{\mathrm{L}}$, as indicated, corresponding to those indicated by the matching symbols in Fig. 1c. Inset, dynamical Coulomb blockade renormalization of $G_{S E T}$ versus d.c. voltage $V_{\mathrm{dc}}$ in the absence of charge quantization, at $\tau_{\mathrm{L}}=0.24$ and $\tau_{R}=1.5$. The error bars are the standard error on the mean value of $\Delta Q$, obtained from the statistical uncertainty of about ten measurements of $G_{\text {SET }}$ (see Methods).

Indeed, the so-called dynamical Coulomb blockade does not rely on a quantized island charge, but results from the discreteness of charge transfers across non-ballistic channels ${ }^{1,2}$.

The degree of charge quantization versus connection strength is characterized, separately from the dynamical Coulomb blockade renormalization of the channels, by focusing on the visibility of the periodic modulations $\Delta Q \equiv\left(G_{\mathrm{SET}}^{\max }-G_{\mathrm{SET}}^{\min }\right) /\left(G_{\mathrm{SET}}^{\max }+G_{\mathrm{SET}}^{\min }\right)$, with $G_{\mathrm{SET}}^{\max (\min )}$ the maximum (minimum) SET conductance over one gate-voltage period and, from now on, $V_{\mathrm{dc}}=0$. A visibility $\Delta Q=1(\Delta Q=0)$ signals a full (an absence of) charge quantization. Moreover, the visibility $\Delta Q$ is directly proportional to the charge oscillations of the island with gate voltage (that is, charge quantization) when one channel approaches the ballistic limit (for example, $\left.\tau_{\mathrm{R}} \rightarrow 1\right)^{7,25-27}$. As put forward in ref. 26 , this proportionality coefficient reduces to the numerical factor $e /(2 \pi \times 1.59)$ for $\tau_{\mathrm{L}} \ll 1$ and $k_{\mathrm{B}} T \ll E_{\mathrm{C}}$.

Figure $2 \mathrm{~b}$ shows $\Delta Q$ versus $\tau_{\mathrm{R}}$ at $T \approx 17 \mathrm{mK}$, with each set of symbols corresponding to a different tuning of the second QPC $\left(\tau_{\mathrm{L}} \in\{0.075\right.$, $0.24,0.49,0.75,0.975,0.983\})$. The robustness of charge quantization with the connection strength of one channel $\left(\tau_{R}\right)$ is established independently of the second channel $\left(\tau_{\mathrm{L}}\right)$, from the nearly constant $\Delta Q$ for $\tau_{\mathrm{R}} \lesssim 0.6$. When further increasing $\tau_{\mathrm{R}}, \Delta Q$ noticeably diminishes and systematically collapses to zero precisely at the ballistic critical point $\tau_{\mathrm{R}}=1$. For $\tau_{\mathrm{R}} \geq 1$, in the presence of one ballistic channel, $\Delta Q$ remains perfectly null at experimental accuracy (see Methods for additional tests).

Power laws characterizing the scaling of charge quantization as $\tau_{\mathrm{R}} \rightarrow 1$ are best revealed by plotting $\Delta Q$ versus the 'distance' from the ballistic critical point $1-\tau_{\mathrm{R}}>0$ on a log-log scale. As shown in Fig. 3, the $T=17 \mathrm{mK}$ data (symbols) systematically vanish as $\sqrt{1-\tau_{\mathrm{R}}}$ (straight lines) for $1-\tau_{\mathrm{R}} \lesssim 0.02$.
Figure 3 Charge quantization scaling near the ballistic critical point. The $\Delta Q$ data at $T \approx 17 \mathrm{mK}$ are displayed versus $1-\tau_{\mathrm{R}}$ on a $\log -\log$ scale lines are quantitative predictions (no fit parameters) derived assuming (bottom three (purple, green and orange) lines). The power law $\Delta Q \propto \sqrt{1-\tau_{\mathrm{R}}}$ (straight, dashed lines) is systematically observed for $1-\tau_{\mathrm{R}} \leq 0.02$ and at intermediate $\tau_{\mathrm{L}}$. The horizontal error bars arise from the dispersion of at least 40 transmission settings; the vertical error bars are calculated from the statistical uncertainty of about 10 measurements of one period of $G_{\text {SET }}$ (see Methods).

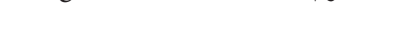



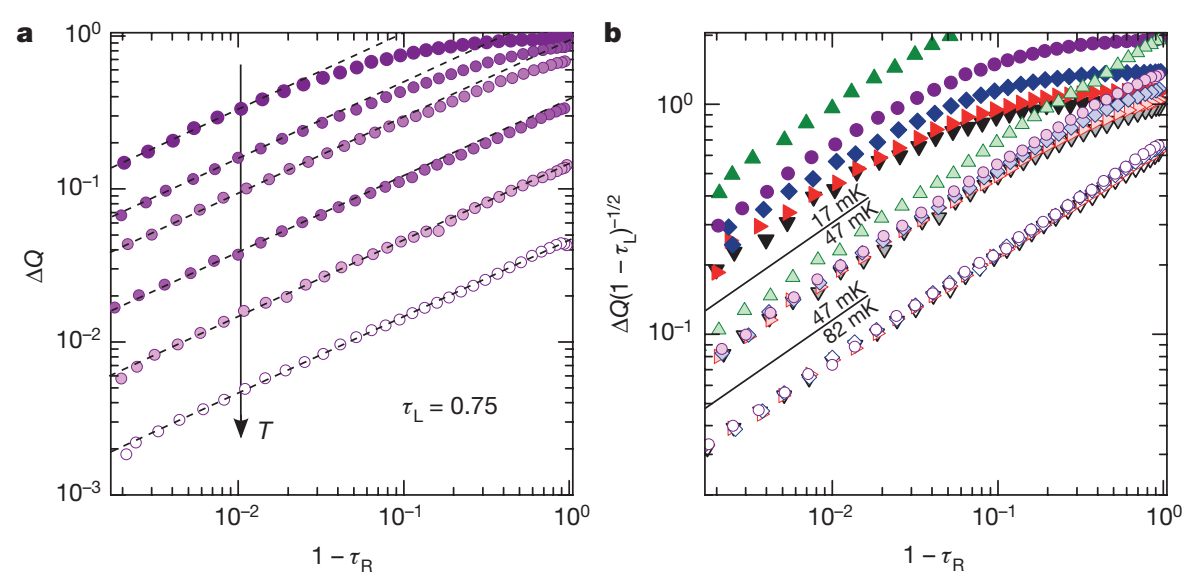

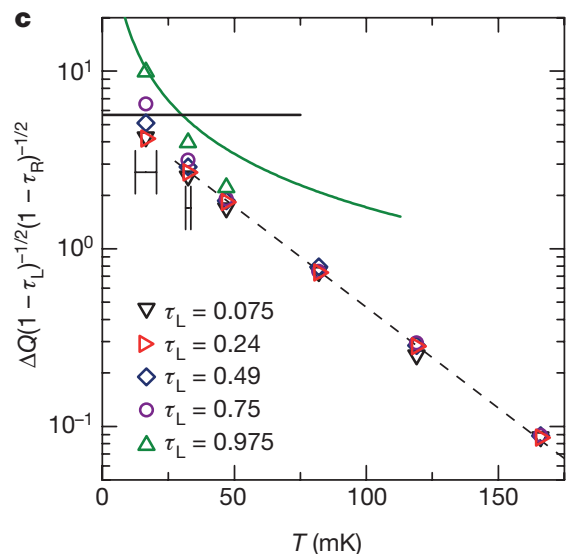

Figure 4 | Crossover to a universal charge quantization scaling as temperature is increased. a, Symbols display $\Delta Q$ versus $1-\tau_{\mathrm{R}}$ at $\tau_{\mathrm{L}}=0.75$ and for $T \approx 17 \mathrm{mK}, 32 \mathrm{mK}, 47 \mathrm{mK}, 82 \mathrm{mK}, 119 \mathrm{mK}$ and $166 \mathrm{mK}$, from top to bottom. The $\tau_{\mathrm{R}}$ range over which $\Delta Q \propto \sqrt{1-\tau_{\mathrm{R}}}$ (straight, dashed lines) extends up to the full interval $\tau_{\mathrm{R}} \in[0,1]$ when increasing $T$. b. The rescaled $\Delta Q / \sqrt{1-\tau_{L}}$ is shown versus $1-\tau_{\mathrm{R}}$, with a different set of symbols corresponding to different $\mathrm{QPC}_{\mathrm{L}}$ set-points as in $\mathbf{c}$. Solid lines separate the data at $T \approx 17 \mathrm{mK}$ (top, darker filling), $T \approx 47 \mathrm{mK}$ (middle) and $T \approx 82 \mathrm{mK}$ (bottom, brighter filling). At $T=82 \mathrm{mK}$, all the data collapse onto a single universal curve $\Delta Q \propto \sqrt{\left(1-\tau_{\mathrm{L}}\right)\left(1-\tau_{\mathrm{R}}\right)}$. $\mathrm{c}$, Symbols

The Coulomb blockade theory of electronic transport in the presence of a nearly ballistic channel $\left(1-\tau_{\mathrm{R}} \ll 1\right)$ relies on the bosonization approach that was initially developed to address correlated electrons in one dimension. Quantitative predictions were obtained for $k_{\mathrm{B}} T \ll E_{\mathrm{C}}$ and for a second channel in either the tunnel $\left(\tau_{\mathrm{L}} \ll 1\right)$ or almost-ballistic $\left(1-\tau_{\mathrm{L}} \ll 1\right)$ regime ${ }^{25,28}$. In both cases, $\Delta Q$ is expected to vanish as $\sqrt{1-\tau_{\mathrm{R}}}$ :

$$
\begin{gathered}
\Delta Q\left(1-\tau_{\mathrm{R}} \ll 1 ; \tau_{\mathrm{L}} \ll 1, k_{\mathrm{B}} T \ll E_{\mathrm{C}}\right) \approx 5.7 \sqrt{1-\tau_{\mathrm{R}}} \\
\Delta Q\left(\sqrt{1-\tau_{\mathrm{L}, \mathrm{R}}} \ll \frac{k_{\mathrm{B}} T}{E_{\mathrm{C}}} \ll 1\right) \approx \frac{0.57 E_{\mathrm{C}}}{k_{\mathrm{B}} T} \sqrt{\left(1-\tau_{\mathrm{L}}\right)\left(1-\tau_{\mathrm{R}}\right)}
\end{gathered}
$$

Such a scaling, initially proposed in ref. 6 , was also predicted for the gate-voltage modulation of thermodynamic quantities for multi-channel junctions using an extension ${ }^{8}$ of the instanton technique ${ }^{1,29}$.

The data establishes the $\sqrt{1-\tau_{\mathrm{R}}}$ scaling for arbitrary $\tau_{\mathrm{L}} \in[0,1]$, beyond the tunnel and ballistic limits currently accessible to transport theory. The dashed lines in Fig. 3 display the asymptotic $\left(\sqrt{1-\tau_{\mathrm{L}, \mathrm{R}}} \ll k_{\mathrm{B}} T / E_{\mathrm{C}}\right)$, quantitative predictions of equation (2) for our completely characterized device at $T=17 \mathrm{mK}$, without fitting parameters. The non-asymptotic $\Delta Q$ predictions (equation (1) for $\tau_{\mathrm{L}} \ll 1$; see Methods for $\left.1-\tau_{\mathrm{L}} \ll 1\right)$ are shown versus $1-\tau_{\mathrm{R}}<0.25$ as solid lines. Data and quantitative predictions are indistinguishable for $1-\tau_{\mathrm{R}} \lesssim 0.1$ for $\tau_{\mathrm{L}}=0.983, \tau_{\mathrm{L}}=0.975$ and, more surprisingly, $\tau_{\mathrm{L}}=0.75$. The equation (1) prediction (black line in Fig. 3) remains noticeably (about $25 \%$ ) above the $\tau_{\mathrm{L}}=0.075$ data for $1-\tau_{\mathrm{R}} \ll 1$. This numerical difference could result from the finite experimental $T$, because equation (1) is exact only at $T=0$.

We now investigate the ways in which the combination of thermal and quantum fluctuations impacts the quantization of charge. As temperature rises, the population of additional charge states is expected to average out charge quantization ${ }^{1,2}$. Figure 4 a displays the measured $\Delta Q$ (symbols) versus $1-\tau_{\mathrm{R}}$ at different temperatures, from $T=17 \mathrm{mK}$ (darker filling) to $T=166 \mathrm{mK}$ (brighter filling), for the representative $\mathrm{QPC}_{\mathrm{L}}$ setting $\tau_{\mathrm{L}}=0.75$. As naively expected, $\Delta Q$ decreases as $T$ increases. In line with thermodynamic expectations ${ }^{8}$ (Methods), the $\Delta Q \propto \sqrt{1-\tau_{\mathrm{R}}}$ scaling (straight lines) that originates from quantum display the fully rescaled data $\Delta Q / \sqrt{\left(1-\tau_{\mathrm{L}}\right)\left(1-\tau_{\mathrm{R}}\right)}$ versus $T$ on semi-log scale, extracted in the regime in which $1-\tau_{\mathrm{R}}$ is small enough that $\Delta Q \propto \sqrt{1-\tau_{\mathrm{R}}} ;$ data for $\tau_{\mathrm{L}}=0.975$ are plotted only for $T \leq 47 \mathrm{mK}$. Horizontal error bars represent the experimental temperature uncertainty at $T=17 \pm 4 \mathrm{mK}$ and $T=32 \pm 1 \mathrm{mK}$. Solid lines are the quantitative predictions in the quantum regime $k_{\mathrm{B}} T \ll E_{\mathrm{C}}$, given by equation (1) (black, horizontal) and equation (2) (green, curved). The straight dashed line displays an exponential decay close to predictions in the presence of strong thermal fluctuations (see text).

fluctuations not only persists for increasing $T$, but extends over a widening range of $\tau_{\mathrm{R}}$, up to the full-scale $\tau_{\mathrm{R}} \in[0,1]$.

The crossover towards this universal behaviour is established by comparing the rescaled visibility $\Delta Q / \sqrt{1-\tau_{\mathrm{L}}}$ for different $\tau_{\mathrm{L}}$ settings with $1-\tau_{\mathrm{R}}$. The symbols in Fig. $4 \mathrm{~b}$ represent the rescaled data at $T=17 \mathrm{mK}, T=47 \mathrm{mK}$ and $T=82 \mathrm{mK}$, with brighter filling at higher temperatures. As $T$ increases, the scatter associated with the various $\tau_{\mathrm{L}}$ values narrows. For $T \geq 82 \mathrm{mK}$, the rescaled data collapse onto a single, universal (for all $\tau_{\mathrm{L}}$ ), straight line $\Delta Q \propto \sqrt{\left(1-\tau_{\mathrm{L}}\right)\left(1-\tau_{\mathrm{R}}\right)}$ over the full range $\tau_{\mathrm{L}, \mathrm{R}} \in[0,1]$.

The temperature dependence is further characterized by plotting $\Delta Q / \sqrt{\left(1-\tau_{\mathrm{L}}\right)\left(1-\tau_{\mathrm{R}}\right)}$ (determined at low enough $1-\tau_{\mathrm{R}}$ such that $\Delta Q \propto \sqrt{1-\tau_{\mathrm{R}}}$ ) versus temperature on a semi-log scale (Fig. $4 \mathrm{c}$, symbols). The $k_{\mathrm{B}} T \ll E_{\mathrm{C}}$ prediction of equation (1) (equation (2)) is displayed as a black (green) solid line for $T<75 \mathrm{mK}(T<115 \mathrm{mK})$. We find for $T \geq 82 \mathrm{mK}$ (up to $166 \mathrm{mK}, 2.8 \leq \pi^{2} k_{\mathrm{B}} T / E_{\mathrm{C}} \leq 5.6$ ) that the different $\tau_{\mathrm{L}}$ data points collapse onto the same exponential decay (dashed line in Fig. $4 \mathrm{c}): \Delta Q \approx \sqrt{\left(1-\tau_{\mathrm{L}}\right)\left(1-\tau_{\mathrm{R}}\right)} \exp \left(-0.8 \pi^{2} k_{\mathrm{B}} T / E_{\mathrm{C}}\right)$. We have extended the Coulomb blockade theory for the conductance to include thermal fluctuations in the limits of tunnel or nearly ballistic channels (Methods). In the regime of strong thermal averaging, we predict $\Delta Q \propto \sqrt{\left(1-\tau_{\mathrm{L}}\right)\left(1-\tau_{\mathrm{R}}\right)} \exp \left(-\pi^{2} k_{\mathrm{B}} T / E_{\mathrm{C}}\right)$ (neglecting factors not exponential in $T$ ) - a dependence that is also expected for thermodynamic properties ${ }^{8}$ (Methods) - in close agreement with the experimental findings regarding the effect of $\tau_{\mathrm{L}, \mathrm{R}}$ and $T$.

Although theoretical predictions for low-temperature transport currently apply to only the nearly ballistic and tunnel limits, we anticipate that recent advances, including those in numerical renormalization group methods ${ }^{30}$, will open up access to the full range of connection strengths. Our results may therefore provide a test-bed for strongly correlated electron-theoretical methods, for which non-perturbative techniques are ubiquitous. The understanding and on-demand control of charge quantization in mesoscopic circuits might lead to applications beyond the field of single electronics. The central role of charge quantization in the different quantum laws of electricity with coherent conductors indicates that direct quantum engineering could have implications for future nanoelectronics, such as semiconductor-metal hybrid devices that are crucial for developing topologically protected quantum bits 9 . The hybrid implementation we have presented also enables further 
fundamental exploration, including of charge quantization with correlated electrons such as in the multi-channel Kondo regime and/or with fractionally charged anyonic quasiparticles.

Online Content Methods, along with any additional Extended Data display items and Source Data, are available in the online version of the paper; references unique to these sections appear only in the online paper.

\section{Received 25 March; accepted 10 June 2016.}

1. Schön, G. \& Zaikin, A. D. Quantum coherent effects, phase transitions, and the dissipative dynamics of ultra small tunnel junctions. Phys. Rep. 198, 237-412 (1990).

2. Grabert, H. \& Devoret, M. H. (eds). Single Charge Tunneling: Coulomb Blockade Phenomena in Nanostructures (Plenum, 1992).

3. Likharev, K. K. Single-electron devices and their applications. Proc. IEEE 87, 606-632 (1999).

4. Meschke, M., Engert, J., Heyer, D. \& Pekola, J. P. Comparison of Coulomb blockade thermometers with the International Temperature Scale PLTS-2000. Int J. Thermophys. 32, 1378-1386 (2011)

5. Pekola, J. P. et al. Single-electron current sources: toward a refined definition of the ampere. Rev. Mod. Phys. 85, 1421-1472 (2013).

6. Flensberg. K. Capacitance and conductance of mesoscopic systems connected by quantum point contacts. Phys. Rev. B 48, 11156-11166 (1993).

7. Matveev, K. A. Coulomb blockade at almost perfect transmission. Phys. Rev. B 51, 1743-1751 (1995).

8. Nazarov, Y. V. Coulomb blockade without tunnel junctions. Phys. Rev. Lett. 82 1245-1248 (1999).

9. Albrecht, S. M. et al. Exponential protection of zero modes in Majorana islands. Nature 531, 206-209 (2016)

10. Kouwenhoven, L. et al. Single electron charging effects in semiconductor quantum dots. Z. Phys. B 85, 367-373 (1991)

11. Staring, A. A. M., Williamson, J. G., van Houten, H. \& Beenakker, C. W. J. Coulomb-blockade oscillations in a quantum dot. Physica B 175, 226-230 (1991).

12. van der Vaart, N. et al. Charging effects in quantum dots at high magnetic fields. Physica B 189, 99-110 (1993).

13. Molenkamp, L. W., Flensberg, K. \& Kemerink, M. Scaling of the Coulomb energy due to quantum fluctuations in the charge on a quantum dot. Phys. Rev. Lett. 75, 4282-4285 (1995).

14. Joyez, P., Bouchiat, V., Esteve, D., Urbina, C. \& Devoret, M. H. Strong tunneling in the single-electron transistor. Phys. Rev. Lett. 79, 1349-1352 (1997)

15. Chouvaev, D., Kuzmin, L. S., Golubev, D. S. \& Zaikin, A. D. Strong tunneling and Coulomb blockade in a single-electron transistor. Phys. Rev. B 59, 10599-10602 (1999)

16. Berman, D., Zhitenev, N. B., Ashoori, R. C. \& Shayegan, M. Observation of quantum fluctuations of charge on a quantum dot. Phys. Rev. Lett. 82 161-164 (1999).

17. Duncan, D. S., Livermore, C., Westervelt, R. M., Maranowski, K. D. \& Gossard, A. C. Direct measurement of the destruction of charge quantization in a single-electron box. Appl. Phys. Lett. 74, 1045-1047 (1999).
18. Amasha, S. et al. Coulomb blockade in an open quantum dot. Phys. Rev. Lett. 107, 216804 (2011).

19. Pasquier, C. et al. Quantum limitation on Coulomb blockade observed in a 2D electron system. Phys. Rev. Lett. 70, 69-72 (1993).

20. Crouch, C. H., Livermore, C., Westervelt, R. M., Campman, K. L. \& Gossard, A. C. Coulomb oscillations in partially open quantum dots. Superlattices Microstruct. 20, 377-381 (1996).

21. Liang, C.-T. et al. Experimental evidence for Coulomb charging effects in an open quantum dot at zero magnetic field. Phys. Rev. Lett. 81, 3507-3510 (1998).

22. Cronenwett, S. M. et al. Mesoscopic Coulomb blockade in one-channe quantum dots. Phys. Rev. Lett. 81, 5904-5907 (1998).

23. Tkachenko, O. A. et al. Coulomb charging effects in an open quantum dot device. J. Phys. Condens. Matter 13, 9515-9534 (2001)

24. Aleiner, I. L. \& Glazman, L. I. Mesoscopic charge quantization. Phys. Rev. B 57, 9608-9641 (1998)

25. Furusaki, A. \& Matveev, K. A. Theory of strong inelastic cotunneling. Phys. Rev. B 52, 16676-16695 (1995).

26. Yi, H. \& Kane, C. L. Coulomb blockade in a quantum dot coupled strongly to a lead. Phys. Rev. B 53, 12956-12966 (1996).

27. Le Hur, K. \& Seelig, G. Capacitance of a quantum dot from the channelanisotropic two-channel Kondo model. Phys. Rev. B 65, 165338 (2002).

28. Matveev, K. A. \& Andreev, A. V. Thermopower of a single-electron transistor in the regime of strong inelastic cotunneling. Phys. Rev. B 66, 045301 (2002).

29. Korshunov, S. E. Coherent and incoherent tunneling in a Josephson junction with a "periodic" dissipation. JETP Lett. 45, 434-436 (1987).

30. Mitchell, A. K., Landau, L. A., Fritz, L. \& Sela, E. Universality and scaling in a charge two-channel Kondo device. Phys. Rev. Lett. 116, 157202 (2016).

Acknowledgements This work was supported by the European Research Council (ERC-2010-StG-20091028, no. 259033), the French RENATECH network, the national French programme 'Investissements d'Avenir' (Labex NanoSaclay, ANR-10-LABX-0035), the US Department of Energy (DE-FG02-08ER46482) and the Swiss National Science Foundation.

Author Contributions S.J. and Z.I. performed the experiment with inputs from A.A. and F.P.; S.J., Z.I., A.A. and F.P. analysed the data; F.D.P. fabricated the sample and contributed to a preliminary experiment; U.G., A.C. and A.O. grew the 2DEG; I.P.L., E.I., E.V.S. and L.I.G. developed the strong thermal fluctuations theory; F.P. led the project and wrote the manuscript with inputs from all authors.

Author Information Reprints and permissions information is available at www.nature.com/reprints. The authors declare no competing financial interests. Readers are welcome to comment on the online version of the paper. Correspondence and requests for materials should be addressed to F.P. (frederic.pierre@lpn.cnrs.fr).

Reviewer Information Nature thanks Y. Nazarov and the other anonymous reviewer(s) for their contribution to the peer review of this work. 


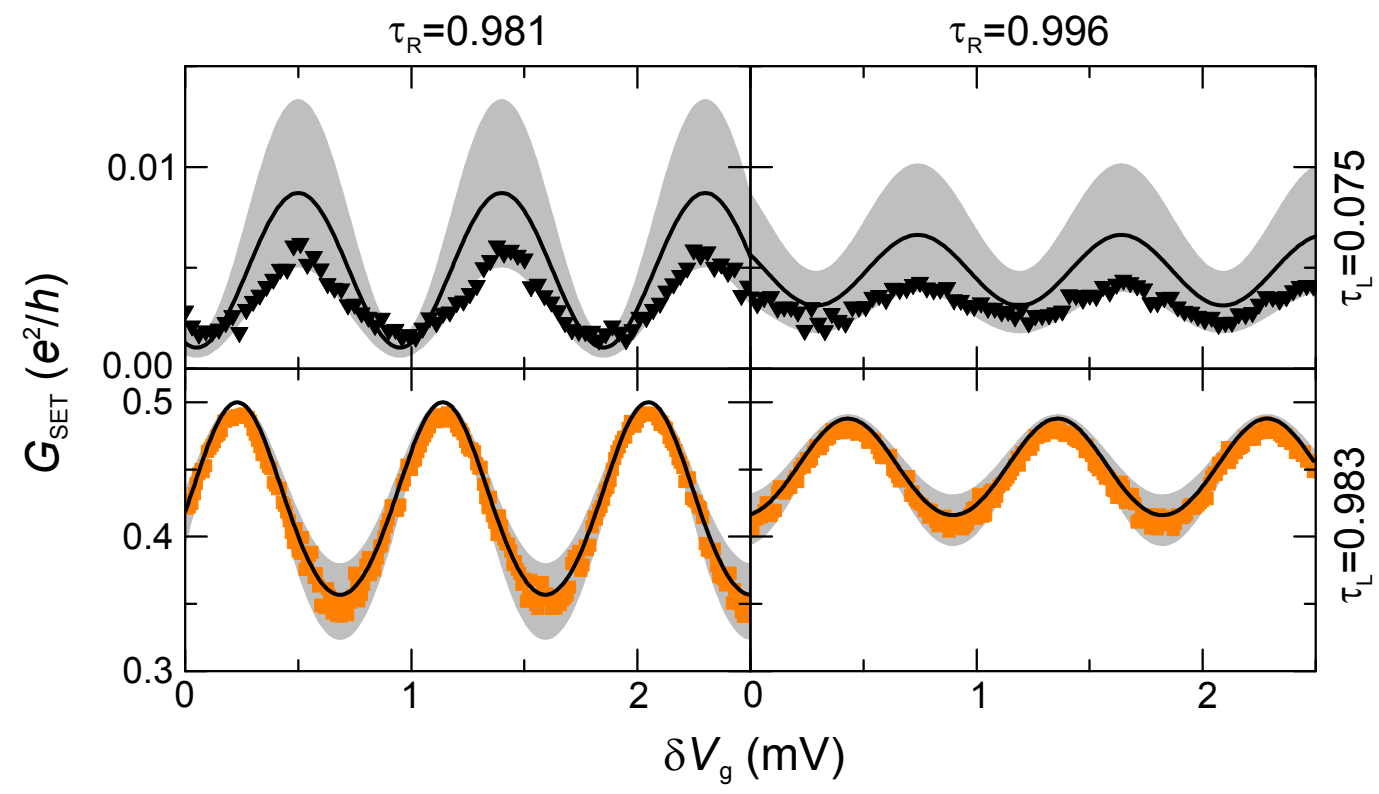

Figure 2.6: Conductance measurements versus quantitative predictions Direct $G_{\mathrm{SET}}\left(\delta V_{g}\right)$ comparison at $T=17 \mathrm{mK}$ between data (symbols) and predictions (solid lines, gray areas correspond to the temperature uncertainty of $\pm 4 \mathrm{mK}$ ) in the two limits addressed by theory (Eq. 2.5) for $\tau_{L} \approx 0$ (top panels), Eq. 2.9] for $\tau_{L} \approx 1$ (bottom panels)).

\subsection{Conclusion}

The use of a hybrid metal-semiconductor device allowed us to probe and control the degree of charge quantization in the metallic nodes of mesoscopic circuits with an unprecedented accuracy. We first verified that charge quantization is completely destroyed as soon as a ballistic channel connects the metallic island/node to the circuit.

At low temperature we compared our experimental data to the quantitative theory in the two known limits $\left(\tau_{L} \ll 1\right.$ and $1-\tau_{R} \ll 1$ called 'asymmetric'; $1-\tau_{L, R} \ll 1$ called 'symmetric') and we observed a quantitative agreement, within the error bars in the symmetric strong coupling limit. In these two known limits, the visibility on the Coulomb oscillations of the conductance was predicted to scale as $\sqrt{1-\tau_{R}}$, we actually observed such a scaling for all intermediate transmissions $0<$ $\tau_{L}<1$ in the low temperature quantum regime. This $\sqrt{1-\tau}$ scaling is expected by the theory at high temperatures $k_{B} T \gg E_{C} / \pi^{2}$ as well as the exponential suppression of quantization with temperature that we observed. Moreover, at high temperature, as expected by theory, the degree of charge quantization adopts a fully 
universal behavior whatever $0<\tau_{L, R}<1$, when rescaled with $\sqrt{1-\tau_{L}} \sqrt{1-\tau_{R}}$. 


\section{Chapter 3}

\section{Observation of the multi-channel 'charge' Kondo effect}

This chapter is divided in three sections. The first one gives an introduction to the multi-channel Kondo effect, which occurs when a local spin is antiferromagnetically coupled with multiple electron continua. It has become central to study non-Fermi liquid physics, but its experimental observations remained mostly elusive. A powerful implementation called 'charge' Kondo effect will be explained in the second section. The charge model involves 'charge' degrees of freedom instead of 'spin'. The community was doubtful about an experimental realization of this model since it requires apparently contradicting design specifications. This was resolved by implementing the 'charge' Kondo model with a hybrid metalsemiconductor device, and gave us access to the rich multi-channel Kondo physics that will be presented in the last section.

\subsection{The Kondo model: a testbed for correlated physics}

The simple Kondo model has attracted a lot of interest because of the associated rich correlated physics. In its original (one-channel) version, it involves a single bath of conduction electrons that tries to screen a magnetic impurity. This model has developed into a testbed for the many-body theoretical methods, in particular the renormalization group. In this picture, as the temperature is lowered, the parameters of the model effectively renormalize to eventually reach universal fixed points, which do not depend on microscopic details.

This renormalization flow takes place on a characteristic temperature called the Kondo temperature $T_{K}$. Although at $T \sim T_{K}$ the one-channel Kondo model involving conduction electrons interacting with a spin-half impurity constitute a many-body problem, at lower temperatures $T \ll T_{K}$, the system behave as a Fermi 
liquid because the impurity is screened.

In an attempt to describe real metals, Nozières and Blandin proposed a variant of the original model where the conduction electrons carry multiple 'flavors' that account for additional degrees of freedom. When the number of flavors is larger than twice the spin of the impurity, the impurity is overscreened. This situation results in an antiferromagnetically coupled residual spin on the impurity site; and hence, in a "residual" Kondo effect. As the Kondo impurity is never fully screened, whatever the temperature, a Fermi liquid behavior is never recovered. The multiple channel/flavor Kondo model therefore gives rise to non-Fermi liquid behaviors.

The first part of this section deals with the original Kondo model (where only a single channel is considered) whereas the second part will be about the multi-channel Kondo model.

\subsubsection{The original Kondo model}

This part starts with a description of the model. Then several solutions will be given, following the historical theoretical developments, in order to eventually get a good understanding of the model. We will finally present some experimental realizations in the realm of nano-devices.

\section{The Kondo model and its solution in the perturbative regime}

The original model was used by Kondo in 1964 [62] to explain a minimum in the 'resistivity versus temperature' curve of some dilute magnetic alloys (for instance AuFe: gold metal with small amounts of iron impurities). In normal metals, the main contribution to the resistivity is the electron-phonon scattering, a contribution that decreases with the temperature. At zero temperature, the phonon population is zero and the finite residual resistivity is explained by the scattering with the defects of the metal. In contrast, the Kondo model reproduces the actual experimental observations 11 of a logarithmic increase of the resistivity at low temperature due to the scattering by magnetic impurities.

The Hamiltonian of the model is [62]:

$$
H_{K}=\sum_{\vec{k}, \sigma} \varepsilon_{\vec{k}} c_{\vec{k}, \sigma}^{\dagger} c_{\vec{k}, \sigma}+J \vec{S} \cdot \sum_{\vec{k}, \overrightarrow{k^{\prime}, \sigma, \sigma^{\prime}}} c_{\vec{k}, \sigma}^{\dagger}(\overrightarrow{0}) \vec{s}_{\sigma \sigma^{\prime}} c_{\vec{k}^{\prime}, \sigma^{\prime}}(\overrightarrow{0})
$$

The first term corresponds to the kinetic energy of the conduction electrons while the second one describes the scattering with a magnetic impurity of spin $\vec{S}$ localized at the origin $(\overrightarrow{0})$. The strength of the interaction between the impurity $\vec{S}$

\footnotetext{
${ }^{1}$ The first observations were made in the 30 's, for a review see [118].
} 
and the conduction electrons is set by the exchange coupling $J$. The $c_{\vec{k}, \sigma}\left(c_{\vec{k}, \sigma}^{\dagger}\right)$ are operators that annihilate (create) a conduction electron with momentum $\vec{k}$ and spin $\sigma$, and $\vec{s}$ is a vector of the Pauli matrices.

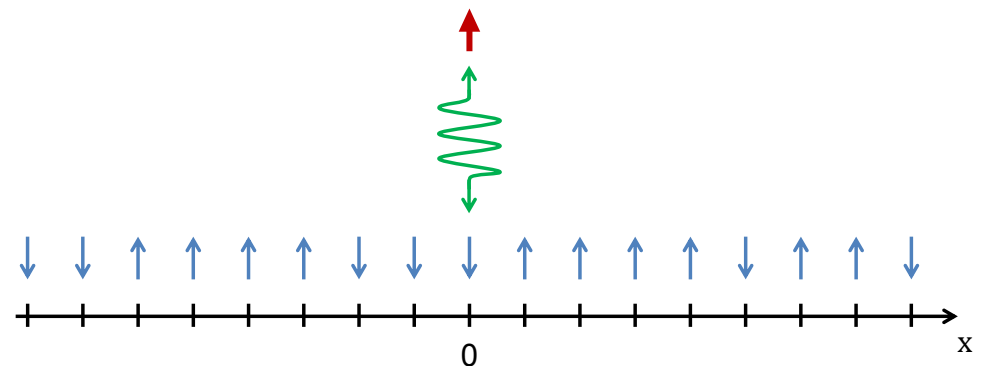

Figure 3.1: Diagram explaining the Kondo model. The impurity $S=1 / 2$ is represented by a red arrow. The conduction electrons (blue arrows) are distributed on a lattice. To simplify, the lattice is 1D. The antiferromagnetic interaction is drawn as a squiggly green arrow located on the impurity site.

In the Kondo Hamiltonian, the coupling term acts only on the site of the impurity (located by convention at the origin $\overrightarrow{0}$, see Fig. 3.1). This coupling is called 'ferromagnetic' when $J<0$, in which case the energy $H_{K}$ is lower when the spin of the electron on the impurity site is parallel to $\vec{S}$. We will rather be interested in the opposite case, $J>0$, of an 'antiferromagnetic' coupling. In this thesis we will only consider the case of a spin-half impurity, $S=1 / 2$. Moreover, while the Eq. (3.1) describes an 'isotropic' Kondo effect, we will also discuss the 'anisotropic' case where the coupling along an axis (say $J_{z}$ ) is different from the other ones $\left(J_{\perp} \triangleq J_{x}=J_{y}\right)$.

Note also that the impurity states $\vec{S}$ are degenerate; breaking the degeneracy by applying a magnetic field $h$ (say along the $z$-axis) will generate a term in $h S_{z}$ in $H_{K}$ because one of the two projections of $\vec{S}$ will be favored. However, having a degenerate impurity is essential to obtain a fully developed Kondo effect.

Assuming $J$ is small, Kondo used a perturbation theory to calculate the resistivity due to the scattering with the impurity $\vec{S}$ [62]. Extending the calculation to the third order in perturbation led him to the following expression:

$$
R(T)=R_{0}\left[1-2 J \rho \log \left(\frac{k_{B} T}{D}\right)\right]
$$

where $R_{0}$ is a constant, $\rho$ is the density of states and $D$ is a high energy cutoff. This expression was supposed to remain small (as it has been derived using a perturbation theory), but it diverges at $T \sim 0$. Moreover, such a divergence 
has not been observed experimentally. Actually, Kondo himself was aware that this expression is not valid at low temperature compared to the so-called Kondo temperature: $k_{B} T_{K} \sim D \exp (-1 / J \rho)$.

Although $T_{K}$ has been initially introduced to delimit the validity of the perturbation theory, it will play an important role in this chapter. Indeed it contains all the microscopic details of the model, which is fully determined by this unique parameter. Results expressed in $T / T_{K}$ will be universal in the sense that any observable from any experiment should behave identically when plotted with this rescaled temperature.

\section{Poor man's scaling}

Actually, Kondo succeeded to explain the intriguing feature of the 'resistivity vs temperature' curves observed in the 30 's... but that is just the beginning of the story! Indeed, the complete understanding of the model and, in particular, the problem of what happens around and below $T_{K}$ has fascinated theorists (even "more than is justified by its experimental significance" according to Wilson [131]).

The key idea is renormalization. If we incorporate the $\log$ term into $J$ in Eq. (3.2), we could say that the exchange coupling is effectively renormalized ${ }^{2}$ to bigger values as the temperature decreases. This concept is actually even more general. Anderson has proposed a poor man's scaling consisting in a renormalization of the Hamiltonian to take into account a reduction of the energy bandwidth of the problem [10]. This derivation also is not rigorous, but it allowed Anderson to claim that the coupling $J$ flows towards infinitely large values $J \longrightarrow \infty$ in the antiferromagnetic 3 case.

The fact that $J$ actually flows to infinity or converges to an intermediate fixed point is not obvious. Going to the next order in Anderson's derivation gives an intermediate fixed point, but this is an artifact [46]. The reliable proof that $J$ flows to infinity has been given by Wilson's numerical calculations [131].

\section{The complete solutions}

The log divergence in the expression of the resistivity computed by Kondo is a signature of a lack of characteristic energy (Kondo had to sum the contribution of the possible processes on the full range of energies with the same weight). This is typical of some problems of quantum field theory or (classical) critical phenomena where renormalization group theory is of great help.

\footnotetext{
${ }^{2}$ Everything happens as if the value of $J$ was changing; but note that the true value of the exchange coupling never changes, it is only effectively renormalized. We will then distinguish the 'bare' value $J_{\infty}$ from 'renormalized' value $J$ (both are equal before renormalization).

${ }^{3}$ It flows to zero in the ferromagnetic case, this case can be solved easily [10, 46].
} 
Wilson has implemented an efficient numerical version of the renormalization and solved the Kondo problem [131]. The key idea of his iterative algorithm is to use a logarithmic discretization to capture all the physics of the problem. Note that this not a perturbative method: the result will be exact provided the discretization step is fine enough and the algorithm has converged.

Using this numerical technique, Wilson was able to compute the impurity magnetic susceptibility and its specific heat. But his major result was the demonstration that there is a unique stable fixed point in the one-channel Kondo model: $J \longrightarrow \infty$.

Other exact solutions have been found for the Kondo model by the Betheansatz technique [12, 123]. A partial solution consists in a mapping of the Kondo model on the resonant-level model; at a particular value the anisotropic coupling $J_{z}$ known as the Toulouse point [115], the interaction term of the resonant-level model vanishes and the problem becomes trivial [9] (see also [46]).

\section{A "Fermi-liquid" description at low temperature}

Based on the fact that the antiferromagnetic coupling $J$ to the impurity $\vec{S}$ flows to infinity, Nozières proposed a basic explanation of the physics at low temperature [94]. At zero temperature, the ground state of $H_{K}$ is simply formed with an electron trapped by the impurity, because the coupling with the impurity is infinite. The remaining electrons are free to visit the other sites of the lattice (except the one of the impurity which remains occupied by a trapped electron).

The impurity and its trapped electron form a rigid singlet complex $S^{\prime}=0$. The second term of $H_{K}$ vanishes when one considers the effective spinless impurity. One eventually gets a Fermi liquid and can, for instance, determine the resistivity or the impurity magnetic susceptibility at low temperature [94].

We have a complete understanding of the one-channel Kondo problem on the theoretical point of view: at low temperatures it is described by the Fermi liquid theory and at high temperatures the perturbation theory holds. The crossover between these two regimes appears around the Kondo temperature $T \sim T_{K}$. At these intermediate temperatures, the situation is complicated; however, more sophisticated techniques exist (in particular numerical renormalization). Let us now briefly discuss experimental investigations of the Kondo effect at the nano/micrometer scale.

\section{Observation of Kondo effect due to a single localized magnetic impurity}

There has been a revival of Kondo effect studies [64] as a result of the development of new experimental techniques. In practice, we are now able to explore the Kondo 
effect due to a well-characterized single impurity, allowing for stringent tests of the theoretical many-body methods.

The most direct example is probably the measurement of a localized magnetic impurity with an STM [76, 74]. In reference [76], some cobalt atoms have been deposited on the surface of a clean crystalline gold (111). Since cobalt is magnetic and gold is not, CoAu alloys were known to exhibit Kondo effect. Here the magnetic impurities are at the surface. They are probed with the aid of the STM tip. The microscope is first used to get the topography of the sample and localize the cobalt atoms. When the tip is put close to an impurity, a peak 4 is observed in the differential conductance versus voltage bias. This signature of the Kondo effect appears only in the vicinity of the impurity and at temperature below $T_{K}$. In ref. [76], the Kondo temperature is deduced from a fit of the conductance peak to a model.

Madhavan and co-workers [76] have obtained a Kondo temperature $\left(T_{K} \approx\right.$ $70 \mathrm{~K})$ much lower than the one measured in bulk CoAu alloys $\left(T_{K}>300 \mathrm{~K}\right)$. Indeed, the coupling of the impurity to the conduction electrons is weaker in their sample with impurities on the surface. This experiment is a direct observation of the Kondo effect, but one might be frustrated because this is just 'a single picture' as the coupling is fixed. To further understand this effect, one wants 'a movie' of how the Kondo effect develops depending on the coupling strength. For such a program, one needs tunable devices.

\section{Kondo effect in tunable nano-devices}

Quantum dots can be fabricated by confining a 2DEG in a small region. Such a OD structure has discrete levels, and one can populate each level with electrons. A quantum dot can therefore be seen as an artificial atom. The idea in references [49, 33] is to use the two degenerate spin states of a quantum dot (when it contains an odd number of electron) to observe Kondo effect.

In these experiments, a quantum dot connects two leads (like in a Single electron transistor) and the conductance is measured through the dot (see Fig. 3.2). In contrast to the usual Kondo effect (where it is the resistivity which increases at low temperature), here, the conductance increases at lower temperature [64]. This inverted behavior for the quantum dot comes from the fact that, in general, conduction is not allowed through the dot at low temperature because of Coulomb blockade (when no level of the dot is tuned to the Fermi energy). However, in the presence of the Kondo effect, the electrons in the two leads jointly try to make a singlet with the spin of the dot, thus coupling the two leads and increasing the conductance across the quantum dot [49, 33].

\footnotetext{
${ }^{4}$ The shape of the peak is modified by a Coulomb interaction, but this is well understood [76].
} 


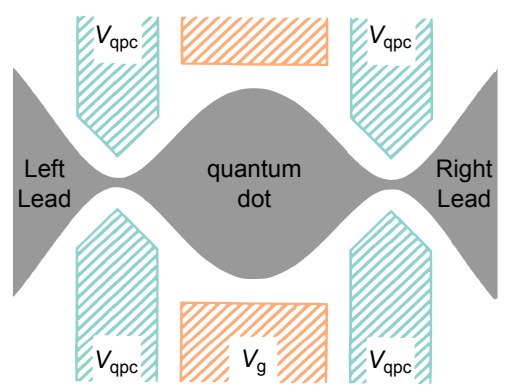

Figure 3.2: Diagram of a quantum dot connected to two leads. A quantum dot formed in a 2DEG (in gray) is connected to two leads through QPCs. The energy in the discrete levels of the dot can be tuned thanks to the gate $V_{g}$.

The demonstration that this increase in the conductance as the temperature is lowered is indeed due to a Kondo effect is that it happens only when the number of electrons in the dot is an odd number. When there is an even number of electrons on the dot, the total quantum dot spin of the ground state is zero, whereas in the odd case it is $S= \pm 1 / 2$.
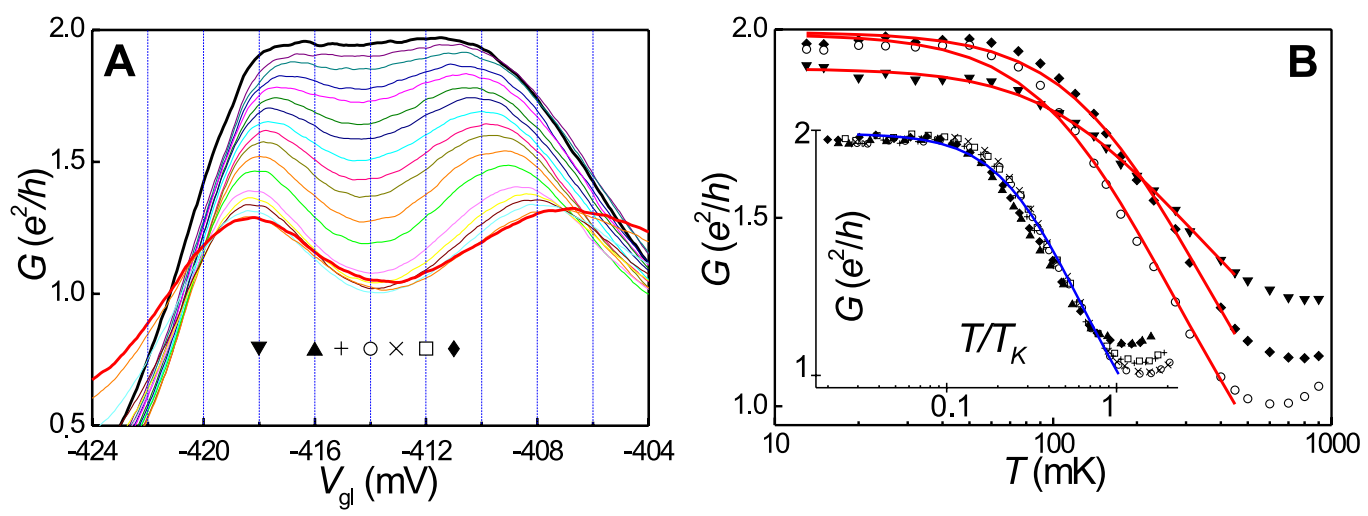

Figure 3.3: Scaling of the conductance $G$ in $T / T_{K}$ (reproduced from [120, 121]). A, Traces of the conductance $G$ versus the lateral gate voltage $V_{\mathrm{gl}}$ for temperatures ranging from $T=15 \mathrm{mK}$ (in black) to $900 \mathrm{mK}$ (in red). B, The conductance is plotted versus temperature for selected values of $V_{\mathrm{gl}}$ (the symbols correspond to $\mathbf{A}$ ). Red lines are fits to empirical law. In the inset, the temperature is rescaled and a universal behavior is observed.

Two years after these pioneer works, in 2000, van der Wiel and co-workers [121] reported an important result (see Fig. 3.3). They measured the conductance versus temperature $G_{\varepsilon_{0}}(T)$ for several settings of $\varepsilon_{0}$, the energy of the last occupied level in the quantum dot with respect to the Fermi energy ( $\varepsilon_{0}$ can be tuned thanks to the lateral gate $V_{\mathrm{gl}}$ ). They then fitted these data to an empirical expression (in red) involving a characteristic temperature called $T_{K}$. When plotting these curves using 
a rescaled temperature $T / T_{K}\left(\varepsilon_{0}\right)$, they observed that some of the curves collapsed onto a single curve. Thanks to a tunable device, van der Wiel and co-workers were therefore been able to check a major feature of the Kondo effect: its universal behavior.

\subsubsection{The multi-channel Kondo model}

The original Kondo model shows interesting features, such as renormalization and (consequently) universal behaviors. However, the impurity is perfectly screened at low temperature leading to a trivial situation. But if one considers more electronic channels (or flavors), the impurity may be overscreened giving rise to non-Fermi behaviors.

In this subsection, we will start with the definition of the multi-channel Kondo model. Then we will explain the existence of a fixed point at finite coupling $J$ when the number of channels $N$ is larger than twice the spin of the impurity: $N>2 S$. This fixed point exhibits fascinating non-Fermi liquid behaviors. This will be followed by a short discussion on strongly correlated materials. And we will finally present the first experimental observations of the multi-channel Kondo effect.

\section{The multi-channel model}

In order to described the Kondo effect in real metals, Nozières and Blandin have proposed a generalization of the original model called the 'multi-channel Kondo model' [93]. This model involves $N$ channels of conduction, whereas the original model was only considering a single channel. Each channel of conduction is independent, just as if the electrons were carrying a flavor (e.g. the orbital degree of freedom). An electron of each flavor can visit each site of the lattice. In particular the impurity site can be occupied by $N$ electrons. As we will soon see, this leads to a completely different qualitative description of the ground state at low temperature. Let me first introduce the Hamiltonian of the multi-channel Kondo model [93]:

$$
H_{N-\mathrm{CK}}=\sum_{a=1}^{N}\left\{\sum_{\vec{k}, \sigma} \varepsilon_{\vec{k}} c_{\vec{k} a \sigma}^{\dagger} c_{\vec{k} a \sigma}+J_{a} \vec{S} \cdot \sum_{\vec{k}, \overrightarrow{k^{\prime}, \sigma, \sigma^{\prime}}} c_{\vec{k} a \sigma}^{\dagger}(\overrightarrow{0}) \vec{s}_{\sigma \sigma^{\prime}} c_{\overrightarrow{k^{\prime}} a \sigma^{\prime}}(\overrightarrow{0})\right\}
$$

The two models are identical except for the channel index $a$, in this model. In particular, the original model is obtained if $N=1$. We can refer to it as ' $1 \mathrm{CK}$ ', while ' $N C K$ ' will refer to the $N$-channel Kondo. Notice that each channel is 
coupled to the impurity $S$ and the channels are not coupled one to another. The coupling of each channel $J_{a}$ may be different.

\section{Intermediate fixed point}

In the $1 \mathrm{CK}$ antiferromagnetic model, even the smallest bare value of $J_{\infty} \neq 0$ will eventually be renormalized to infinity. It means that $J \longrightarrow \infty$ is a stable fixed point while $J=0$ is unstable.

The position of the fixed points in the multi-channel Kondo model strongly depends on the number of channels. Nozières and Blandin [93] have predicted the existence of a fixed point at finite coupling when the number of channels is larger than twice the value of the spin: $N>2 S$. To illustrate their argument, let us consider our spin-half impurity $S=1 / 2$ in the two-channel Kondo model.

First, when $J_{1,2}$ are small, a perturbative approach holds since $T \gg T_{K}$. The $2 \mathrm{CK}$ effect essentially corresponds to twice the $1 \mathrm{CK}$ effect. Indeed, as each channel weakly screens the Kondo impurity, both $J_{1}$ and $J_{2}$ are renormalized independently to larger values.

Second, let us consider the situation where $J_{1}=J_{2} \triangleq J_{2 \mathrm{CK}}$ are infinitely large. In this limit, the impurity traps as many electrons as possible (i.e. the number of trapped electrons equals the number of channels $N=2$, see Fig. 3.4p. The total spin on the impurity site $S^{\prime}=S-N / 2$ is not a singlet in the $2 \mathrm{CK}$ case: the impurity is overscreened. This situation can be modeled with a new antiferromagnetic Kondo effect between $S^{\prime}$ and the conduction electrons with a weak effective coupling $J_{2 \mathrm{CK}}^{\prime}$. We know that such a fixed point is unstable 5 (just as $J=0$ was unstable in the $1 \mathrm{CK}$ model).

In the $2 \mathrm{CK}$ model, both $J_{2 \mathrm{CK}} \longrightarrow \infty$ and $J_{2 \mathrm{CK}}=0$ are therefore unstable. Hence, there should be an intermediate fixed point towards which the symmetric couplings $J_{1}=J_{2}$ are renormalized as the temperature is lowered. This is in contrast with the $1 \mathrm{CK}$ model, where the unique stable fixed point is $J_{1 \mathrm{CK}} \longrightarrow \infty$.

\section{Non-Fermi liquid ground state}

The ground state of the $2 \mathrm{CK}$ intermediate fixed point is qualitatively different from the $1 \mathrm{CK}$ fixed point. In the latter case the impurity is completely screened, this yields a non-magnetic impurity which interacts weakly with the conduction electron at $T \ll T_{K}$. On the other hand, for the $2 \mathrm{CK}$, the impurity is overscreened: The net spin on the impurity site never vanishes, and at low temperature, there

\footnotetext{
${ }^{5}$ In the underscreened case $N<2 S$, the residual effective weak coupling to $S^{\prime}$ is ferromagnetic, the fixed point $J_{\text {underscreened }} \longrightarrow \infty$ is therefore stable. This case has been realized in recent experiments [106, 98], however it is not supposed to lead to a non-Fermi liquid ground state (but rather to a 'singular' Fermi liquid one [83]).
} 


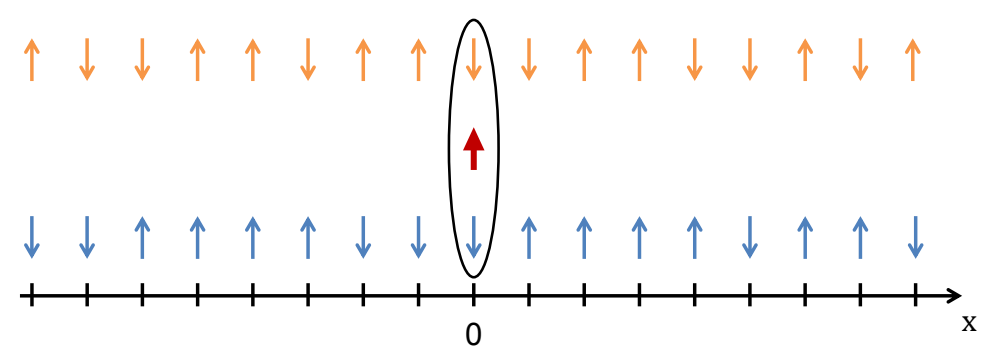

Figure 3.4: Overscreened impurity in the 2CK model. The impurity traps 2 electrons, one of each channel (blue and orange). This complex (encircled with a black line) has a residual spin, in contrast to the $1 \mathrm{CK}$ model. The impurity is overscreened, and this situation is unstable.

are still virtual hoppings of electrons of each channel that compete to screen the effective impurity. This nontrivial ground state has some exotic properties; for instance, the impurity has a finite entropy at zero temperature that is equal to $\ln (2) / 2$ ([39] and references therein).

The properties of this fixed point have been evaluated using several methods (renormalization group [93, 30] or more recently [86, 20], Bethe-ansatz [13, 117] and CFT [116, 3, 4]). As for the original Kondo model, the key quantities to determine are the resistivity, the magnetic susceptibility and the specific heat of the impurity. The exotic properties appear when the number of channel is bigger than twice the impurity spin: $N>2 S$. The $2 \mathrm{CK}$ is the simplest model that realizes an overscreened impurity, and hence, it has become a prototype to study non-Fermi liquid behaviors. Indeed both the spin susceptibility of the impurity $\chi$ and its specific heat coefficient $C_{\mathrm{imp}} / T$ diverge logarithmically (see Bethe-ansatz and conformal field theory techniques references above and also [39, 111]).

Affleck and Ludwig have used CFT techniques to compute the zero-energy amplitude of the single-particle scattering off the impurity [4]. Their expression takes the spin $S$ of the impurity and the number of channels $N$ as parameters. For $S=1 / 2$, this amplitude is equal to 1 for $N=1$, which means that the scattering of a single particle on the Kondo impurity in the $T \longrightarrow 0$ limit simply amounts to a phase shift [18]. In contrast, this amplitude is smaller than 1 for $N>1$, which means that many body collisions remain important even as $T \longrightarrow 0$ in stark contrast to a Fermi liquid description of free quasiparticles [4, 29, 18].

\section{Emergence of exotic quasiparticles}

Majorana fermions are hypothetical spin-half particles predicted by Ettore Majorana to be their own antiparticles. The investigations on Majorana fermions concern 
fundamental research in both particle physics and condensed matter physics [130]. The motivation in the latter field comes from the proposal [105, 61] that, in some particular geometries, the exchange of Majorana fermions involves topological non-commutative properties that could be used to build a fault-tolerant quantum computer [89, 1].

The low-energy collective excitations at the 2CK quantum critical point can be theoretically modeled with Majoranas. Indeed, using the same idea as Toulouse [115], Emery and Kivelson have shown that at the 2CK fixed point the real part of the electronic excitations decouples, leading to a free Majorana fermion [39, 46, 82] (see also [29,27, 26]). One can here mention that the 3CK fixed point might involve Fibonacci anyons rather than Majorana fermions 6 .

Let me emphasize that the conditions for these exotic quasiparticles to emerge in the present multi-channel Kondo effect practical implementations require finetuning (low temperature $T \ll T_{K}$, fully degenerate quantum impurity and no channel symmetry perturbation) in contrast to the robust topological properties required for fault-tolerant quantum computation. These emerging particles can probably not be used for quantum computation.

\section{Kondo effect in some strongly correlated materials}

The ongoing research on some strongly correlated materials and in particular high critical temperature $\left(T_{C}\right)$ superconductor is quite intense. The standard BCS theory sets an upper limit for $T_{C}$ around $30 \mathrm{~K}$. This limit has been exceeded first in 1986 in a material based on copper oxide [15]. To date, the record is $T_{C}=135 \mathrm{~K}$ in normal conditions. The experimental progress raises the hope of practical realization at room temperature. For a recent review on the high-temperature superconductivity in copper oxides, see [59].

Another member in the family of strongly correlated materials are the heavy fermion compounds. In these materials, localized magnetic moments are forming a 'Kondo-lattice'. Heavy fermion compounds can also show non-Fermi liquid physics and superconductivity (up to $T_{C} \approx 20 \mathrm{~K}$ ). The physics in the Kondolattices involves a subtle interplay between, on the one hand the Kondo interaction of the conduction electrons with the localized moments, and on the other hand an RKKY interaction between these moments (for a recent review on heavy fermion compounds, see [44]).

\section{Quantum phase transition}

What we have discussed so far when dealing with the $2 \mathrm{CK}$ overscreened model was the case of two symmetric couplings $J_{1}=J_{2}$. Let us now consider a channel

${ }^{6}$ Private discussion with A.K. Mitchell; the proposal is from L. Fritz. 
asymmetry.

When we introduce an asymmetry between the bare exchange couplings, say $J_{1 \infty}>J_{2 \infty}$, the strongest coupled channel (here channel \#1) will screen the impurity at low temperature [40] (see Fig. 3.5). The ground state will be similar to the 1CK model: a singlet is formed between an electron of channel \#1 and the impurity, and all the other conduction electrons are free. It means that a channel asymmetry in the $2 \mathrm{CK}$ model will lead to a Fermi liquid ground state, while it was non-Fermi liquid in the symmetric case.

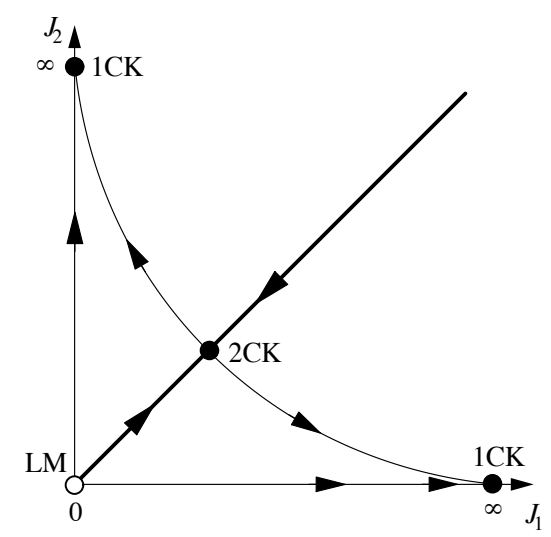

Figure 3.5: Flow diagram of the 2CK model (reproduced from [126]). The arrows are pointing towards low temperatures. This diagram shows the renormalization of the exchange coupling $J_{i}$ of each channel. One can see the finite coupling $2 \mathrm{CK}$ fixed point and the $1 \mathrm{CK}$ fixed points (at infinity). 'LM' is the local-moment fixed point of an uncoupled impurity $\left(J_{1}=J_{2}=0\right)$.

Any finite initial difference between the bare couplings will diverge under the renormalization process [2, 40]. This critical behavior is a quantum phase transition.

The importance of the $2 \mathrm{CK}$ model is partly due to the non-Fermi liquid behaviors and their link with strongly correlated materials and partly due to the richness of the physics of quantum phase transitions. The latter point will be the topic of Chapter 4

\section{First observation of the two-channel Kondo effect}

Despite the fact that the multi-channel Kondo model was originally elaborated to describe the Kondo effect in real metals (with orbital degrees of freedom), no clear evidence of non-Fermi liquid behaviors have been observed in dilute magnetic alloys [40]. The reason for this is probably that there is nothing in these basic systems that forbids a channel asymmetry. A practical application of the 
multi-channel Kondo model came to the fore in the mid-nineties, with a two-level tunneling system [103].

In this experiment the conductance of a point contact between two pieces of metal have been measured. The point contact acts as a two-level system. The tunneling of electrons through this kind of system have been predicted to show Kondo effect, indeed the spin of the impurity can be mapped onto the two-level system [124].

This implementation can succeed in preserving the channel symmetry, and a non-Fermi liquid power law has been observed for the conductance versus temperature or voltage bias, $G(T, V)$. Moreover, a rescaling of the data shows a universal behavior that was predicted by the theory.

However, the device is not tunable since one can neither change the size of the constriction of the point contact nor the symmetry between the channels. The latter point is important in order to observe a crossover from the non-Fermi liquid to the Fermi liquid physics (quantum phase transition). Moreover, the $2 \mathrm{CK}$ interpretation of these results is controversial since the authors of ref. [133] propose another interpretation [104]. For a recent clarification, based on a 2CK model, see [14].

\section{Multi-channel Kondo effect in tunable nano-devices}

We have seen that quantum dots are good systems with which to control the original Kondo effect. To implement the multichannel version, one needs independent baths of electron interacting with the quantum dot. This is very challenging, not on a nano-fabrication point of view but rather on the sample design.

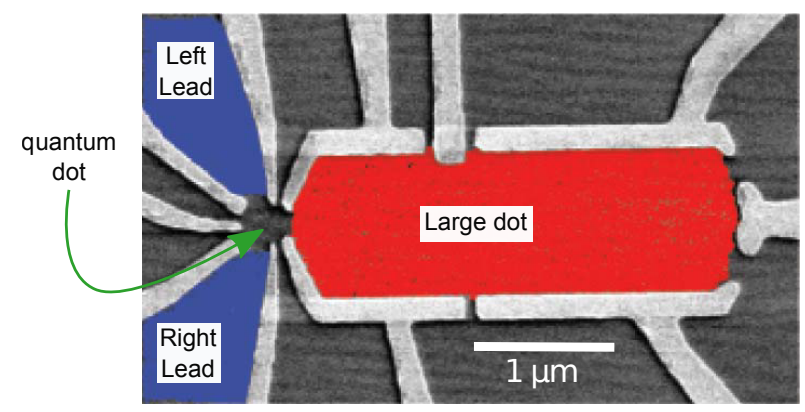

Figure 3.6: Micrograph of the sample used by Potok and co-workers [101]. The 'Left' and the 'Right' leads (in blue) constitute one channel. The large quantum dot (in red) is another independent channel. The quantum dot is at their meeting point (indicated with a green arrow).

In the previous design, two leads were connecting a quantum dot. Each lead is connected to a voltage source and therefore constitutes a bath of electrons. However, since electrons are passing quantum coherently through the dot, there 
is a connection between the two baths. This does not implement the $2 \mathrm{CK}$ but the 1CK model because the two baths of electrons are not independent. Oreg and Goldhaber-Gordon have proposed to add a large quantum dot in the original design [96]. The two first leads will constitute the first bath while the large dot will be the second one (see Fig. 3.6. Side gates are tuned in order to forbid charge transfer to the large dot thanks to Coulomb blockade. The two baths are thus independent and this system can be tuned to non-Fermi liquid.

This design has been implemented by the team of D. Goldhaber-Gordon to observe $2 \mathrm{CK}$ effect [101, 60]. In the first article, they have measured the non-Fermi liquid power law expected [96, 102]. In the second article they have studied the crossover from non-Fermi to Fermi liquid (when breaking the symmetry between the two channels). Their results are in good agreement with the joined NRG calculations. However, the non-metallic character of the large dot introduces a cutoff for the Kondo scaling physics because of the energy level spacing in this dot (which is finite in practice, but should be negligible in theory). The other main limitation of this observation is the absence of quantitative characterization of the Kondo parameters. In this chapter we demonstrate the two- and three-channel Kondo effect based on an idea proposed by Matveev and Glazman in the early nineties and which allows for the perfect knowledge of the Kondo parameters [48, 79].

\subsection{The 'charge' implementation of the Kondo model}

The Kondo model has different implementations. Indeed, one basically needs a two-level system degenerate in energy, in interaction with $N$ continua. But neither the two-level system need necessarily to be based on spin degrees of freedom, nor the continua need to be of fermionic nature! It then exists a plethora of Kondo effects: even a qubit in interaction with a dissipative environment is a Kondo-type system [125, 70].

In this section, we will consider the 'charge' Kondo model, where the degrees of freedom are the charge states of a metallic island weakly coupled to $N$ electrodes with single-mode junctions. Our sample is exactly described by this model which exactly maps onto the multi-channel Kondo model.

We will first explain the analogy between the 'spin' and the 'charge' Kondo implementation, and then we will give some theoretical predictions for the latter model. At the end of this section, we will discuss the practical implementation of the model. 


\subsubsection{Mapping of the Coulomb blockade Hamiltonian onto the Kondo model}

In 1991, Matveev [79] drew an analogy between the problem of a metallic island weakly connected to a massive electrode and the multi-channel Kondo model. An exact correspondence between the Hamiltonian of these two problems was established, provided the validity of a few underlying hypotheses.

In this subsection, we will present the ingredients needed to map the two problems by first considering a single channel. Then we will show that it can be naturally extended to the multi-channel model. We will draw the correspondence between the parameters of each model and give a simple picture that explains the analogy. We will finally show that this mapping goes beyond the tunnel limit condition that was used in its derivation.

\section{A piece of metal close to degenerate charge states}

Let us first consider a metallic island connected to a massive electrode through a single electronic channel only (see Fig. 3.7). The number of electrons on the island can be changed thanks to a lateral gate $V_{g}$ (like in a single electron transistor). Close to the degeneracy point $\delta V_{g}=0$ between two charge states of the island7 7 $Q=0$ and $Q=e$, the Hamiltonian takes the following form [79]:

$$
H=\sum_{k, \alpha} \varepsilon_{k} c_{k \alpha}^{\dagger} c_{k \alpha}\left(\hat{P}_{0}+\hat{P}_{1}\right)+e \delta V_{g} \hat{P}_{1}+t \sum_{k, k^{\prime}}\left(c_{k 1}^{\dagger} c_{k^{\prime} 0} \hat{P}_{0}+c_{k^{\prime} 0}^{\dagger} c_{k 1} \hat{P}_{1}\right)
$$

where $t$ is the tunneling probability, $\alpha$ gives the position of the created / annihilated electron: $\alpha=+1 / 2$ means on the island and $\alpha=-1 / 2$ means in the electrode. The operators $\hat{P}_{\beta}$ are projectors on the eigensubspace of the charge operator $\hat{Q}$, which can take two eigenvalues $\beta e$ with $\beta=0$ or 1 (see below).

In this Hamiltonian, the first term is the sum of the kinetic energies of the electrons on the island and on the electrode, but limited to the states with $Q=0$ or $Q=1$, because we have assumed that we are close to degeneracy $e \delta V_{g} \ll E_{C}$ (other charges states are thus not populated). The second term is the work done by the lateral gate. The last term stands for the tunneling processes: the first term of the parenthesis acts only if $Q=0$, in which case it annihilates an electron of the electrode and creates one in the island; similarly, the second term transfers a charge $e$ from the island to the electrode. One can easily generalize this Hamiltonian to a version with two electrodes, which will accurately describe a SET close to degeneracy.

\footnotetext{
${ }^{7}$ Matveev considers a metallic island, which therefore contains a macroscopic number of electrons. The charge $Q$ we are considering is the charge in excess.
} 


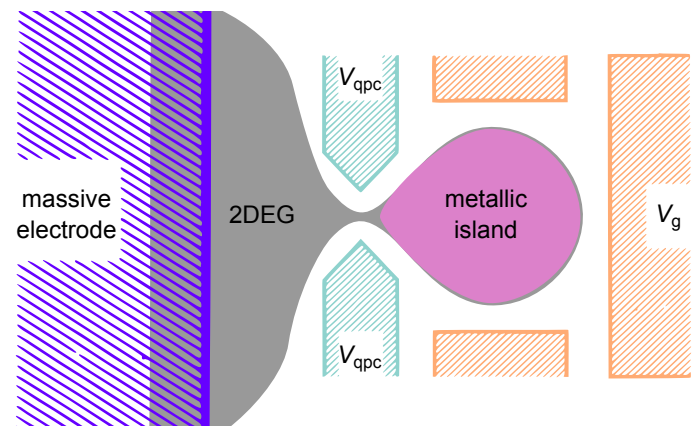

Figure 3.7: Quantum fluctuation of the charge of a metallic island. A massive electrode (in violet) can exchange single electrons with metallic island (in pink) through a single electronic channel made in a 2DEG with a QPC (in the IQHE regime). The plunger gate $V_{g}$ can be tuned to bring the state with $n$ or $n+1$ electrons on the island to the same energy (the island is then at 'charge degeneracy').

\section{Mapping onto the Kondo model}

One can note the similarity of the Coulomb blockade Hamiltonian and the one of the Kondo effect Eq. (3.1). Matveev transformed this Hamiltonian and used Pauli matrices to rewrite it in the form of the anisotropic $\left(J_{z} \neq J_{x}=J_{y} \triangleq J_{\perp}\right)$ Kondo model:

$$
H=\sum_{k, \alpha} \varepsilon_{k} c_{k \alpha}^{\dagger} c_{k \alpha}-2 h S^{z}+\sum_{k, k^{\prime}, \alpha, \alpha^{\prime}} J_{\perp}\left(\sigma^{x} S^{x}+\sigma^{y} S^{y}\right)_{\alpha \alpha^{\prime}} c_{k^{\prime} \alpha^{\prime}} c_{k \alpha}
$$

where $h$ is a magnetic field along the $z$-axis. Let us emphasize here that the 'spin' $\alpha$ involved in this mapping is not the true spin $\sigma$ discussed in the previous Kondo models (the original and the multi-channel). We call this a 'pseudo-spin': it can take only two values as it refers to the position of the electron (either on the island or outside). The impurity $\vec{S}$ and location $\vec{\alpha}$ pseudo-spins are thus necessarily equal to $\pm 1 / 2$.

A simple picture based on pseudo-spins explains this mapping. Indeed, an electron that enters the island flips both the location pseudo-spin $\alpha= \pm 1 / 2$ and the impurity pseudo-spin $S= \pm 1 / 2$. This implements the Kondo process quite naively, with an exchange coupling $J$ directly related to the tunneling amplitude $t$ of the Coulomb blockade model.

This implementation of the Kondo effect will be called the 'charge' Kondo model, in contrast to the original (which we be referred as the 'spin' Kondo model). The degrees of freedom involved in each model are of different nature (charge and spin), but both describe the same Kondo effect. The major advantage of this implementation is the natural access it gives to the multi-channel Kondo 
model as explained below.

\section{A natural implementation of the multi-channel Kondo model}

So far we have considered a single-mode junction between the electrode and the island. However, several electronic channels could participate. There are basically three ways to increase the number of channels: (i) to consider the true spin of the electron and say that two identical channels are connecting the island, (ii) to use a wider junction that allows more transverse modes, (iii) to add more electrodes with single-mode junctions.

All these options are modeled identically because electronic channels are assumed independent in the Landauer-Büttiker formalism. One has just to sum over the number $N$ of channels to get the Coulomb blockade or the 'charge' Kondo Hamiltonian:

$$
\begin{aligned}
H & =\sum_{a=1}^{N} \sum_{k, \alpha} \varepsilon_{k a} c_{k a \alpha}^{\dagger} c_{k a \alpha}\left(\hat{P}_{0}+\hat{P}_{1}\right)+e \delta V_{g} \hat{P}_{1}+t_{a} \sum_{k, k^{\prime}}\left(c_{k a 1}^{\dagger} c_{k^{\prime} a 0} \hat{P}_{0}+c_{k^{\prime} a 0}^{\dagger} c_{k a 1} \hat{P}_{1}\right) \\
H & =\sum_{a=1}^{N} \sum_{k, \alpha} \varepsilon_{k a} c_{k a \alpha}^{\dagger} c_{k a \alpha}-2 h S^{z}+\sum_{k, k^{\prime}, \alpha, \alpha^{\prime}} J_{a \perp}\left(\sigma^{x} S^{x}+\sigma^{y} S^{y}\right)_{\alpha \alpha^{\prime}} c_{k^{\prime} a \alpha^{\prime}} c_{k a \alpha}
\end{aligned}
$$

Thus, there is a correspondence between the parameters of each model. This is summarized in Table 3.1 .

\begin{tabular}{|c|c|c|c|}
\hline \multicolumn{2}{|c|}{ 'spin' Kondo model } & \multicolumn{2}{c|}{ 'charge' Kondo model } \\
\hline impurity spin & $S= \pm 1 / 2$ & charge state & $Q=0$ or $e$ \\
electrons spin & $\sigma=\{\uparrow, \downarrow\}$ & electron position & \{in, out of $\}$ the island \\
Kondo channel & $a=1, \ldots, N$ & electronic channel & $1, \ldots, N$ \\
exchange coupling & $J$ & tunneling probability & $t$ \\
magnetic field & $h$ & gate voltage & $\delta V_{g}$ \\
\hline
\end{tabular}

Table 3.1: Correspondence between the 'spin' and the 'charge' Kondo models

The channel symmetry is an important issue in the multi-channel Kondo model. One needs to master the connection of each individual channel to be able to observe the non-Fermi liquid behaviors associated with an overscreened impurity. The option (ii) is thus not suitable. In the option (i) the two (true) spins are identically connected to the island $\left(t_{\uparrow}=t_{\downarrow}\right)$, and the symmetry of the two Kondo channels is thus guaranteed by construction.

However, the option (iii) is more generic. If one can tune manually each electronic channel to get them symmetric, one will naturally access to the $N$ channel Kondo effect. Moreover, one can break the symmetry between the channels 
and may observe signatures of the expected quantum phase transition. This is actually the option we have used in our experiments.

\section{General validity of the 'charge' Kondo model}

One may have noticed two disadvantages of the 'charge' Kondo implementation compared to its original version: (i) the mapping has been made for low tunneling amplitudes $t$ only and (ii) the equivalent exchange coupling is anisotropic by construction $\left(J_{z}=0\right.$ in the 'charge' Kondo Hamiltonian of Eq. (3.5)).

Firstly, the validity of the mapping goes beyond the tunnel limit $8 \ll 1$, as shown in ref. [78]. In this article, Matveev studied the same problem of the charge quantum fluctuations on a piece of metal coupled to large electrode, but in the opposite limit of an almost perfectly transmitted channel (weak backscattering $1-\tau \ll 1)$. He found a logarithmic divergence of the differential capacitance of the island when approaching the degeneracy $\delta V_{g}=0$. Note that the differential capacitance, $C_{\text {diff }}=\partial Q / \partial V_{g}$, in the 'charge' Kondo model corresponds to the magnetic susceptibility, $\chi=\partial M / \partial h$, of the impurity in the 'spin' Kondo model. His calculations do not require the analogy with the Kondo model, they are rather based on the bosonization technique, and the same logarithmic divergence is found in the tunnel case. This result motivated Matveev [78] to claim that the mapping between the two models is not only valid in the tunnel limit but on the full transmission range $\tau \in[0,1]$.

This prediction has been demonstrated numerically by Lebanon and co-workers [72]. They have implemented the Coulomb blockade Hamiltonian with a true spin (that plays the role of two symmetric channels) and they have observed a $2 \mathrm{CK}$ effect on the capacitance at degeneracy for different values of $t \rho$ (where $\rho$ is a constant density of state) over the full transmission range.

Secondly, the anisotropy of the exchange coupling in the Kondo model is known to be 'irrelevant' for $S=1 / 2$ [2]. In the renormalization group language [58, 132, 131], an 'irrelevant' observable is an observable which flows to weaker values under the renormalization process. This means that the results found using an anisotropic model are not less general since this perturbation will reduce under renormalization.

\section{General requirements to implement the 'charge' Kondo model}

In the scope of a practical implementation, let me list the requirements to observe the 'charge' Kondo effect:

\footnotetext{
${ }^{8}$ With our practical implementation in the QHE regime, a single channel is transmitted through the junction below $\tau=1$. In the tunnel regime, one can identify the transmission with the tunneling probability in this problem $\tau=t^{2} \ll 1$.
} 
(i) continuous density of states in the island (negligible level spacing $\delta E \ll$ $k_{B} T$ ), which is necessary to map the electrons localization onto a pseudospin;

(ii) low temperature and voltage bias compared to the charging energy: $k_{B} T, e V \ll E_{C}$, necessary to reduce the charge state to a pseudo-spin;

(iii) negligible energy dependence of the transmission $\tau(E)$ on the range explored experimentally $|E|<E_{C}$, to avoid additional complications (this can effectively lower the energy bandwidth).

\subsubsection{Theoretical predictions for the 'charge' Kondo model}

We are interested in theoretical predictions to compare with our experimental observations. The main quantity we will deal with is the conductance $G$ per electronic channel connecting the metallic island 9 . As we have already discussed in Chapter 2, there is a quantitative prediction in the case of two channels almost perfectly connected. This case corresponds to the strong coupling limit of the 'charge' 2CK model.

We need predictions for the two- and three-channel 'charge' Kondo model. The conductance $G^{*}$ of each of the symmetric channels at the fixed point has been derived in the general $N$-CK 'charge' Kondo model, with $N \geqslant 2$. As far as I know, there is no prediction for the $1 \mathrm{CK}$, but we will propose a naive guess based on a relation between the exchange coupling $J$ and the transmission $\tau$.

Afterwards we will focus on the power law of the conductance versus temperature near the fixed point, $\left|G-G^{*}\right|(T) \propto\left(T / T_{K}\right)^{\gamma}$ for the 2- and 3-CK. This scaling depends on the transmission $\tau$ of the (symmetric) channels, with $T_{K} \sim E_{C} / \delta \tau^{1 / \gamma}$, where $\delta \tau=\left|\tau-\tau_{C}\right|$ and $\tau_{C}$ is the bare transmission that corresponds to the fixed point $G^{*}$.

\section{The channel conductance and fixed points in the 'charge' Kondo effect}

As explained when discussing the multi-channel Kondo model: knowing the position of the fixed point is a crucial and difficult issue. However, Yi and Kane found an expression that gives the conductance $G^{*}$ at the fixed point of the $N$ channel 'charge' Kondo model, for all $N \geqslant 2[135]$ !

$$
G_{N-\mathrm{CK}}^{*} / G_{K}=2 \sin ^{2} \frac{\pi}{2+N}
$$

\footnotetext{
${ }^{9}$ In this chapter, we will consider the individual conductance $G$ of each QPC and not the serial conductance $G_{\text {SET }}$ through the whole device as in Chapter 2. For instance, with two symmetric channels $G \triangleq G_{1}=G_{2}$, the serial conductance is $G_{\mathrm{SET}}=G_{1} G_{2} /\left(G_{1}+G_{2}\right)=G / 2$.
} 
This formula agrees with the cases $N=2$ and $N=4$ that had already been treated in ref. [43]. Table 3.2 summarizes the position of the fixed point for the first values of the 'charge' $N$-channel Kondo model.

\begin{tabular}{|c|c|c|}
\hline$N$ & $G_{N \mathrm{CK}}^{*} / G_{K}$ & Reference \\
\hline 1 & $0 ?$ & \\
2 & 1 & {$[43,135$} \\
3 & $2 \sin ^{2}(\pi / 5) \approx 0.691$ & {$[135$} \\
4 & $1 / 2$ & {$[43,135$} \\
\hline
\end{tabular}

Table 3.2: Fixed point of the $N$-channel 'charge' Kondo model

The prediction for the $1 \mathrm{CK}$ case is not certain, it is based on a argument explained just below.

\section{A naive reasoning to get $G_{1 \mathrm{CK}}^{*}$ using a $J(\tau)$ relation}

In the mapping of Matveev [79], the exchange coupling $J$ of the Kondo model is associated with the tunneling matrix element $t$ of the Coulomb blockade model by $J=t$. But the link between the tunneling element $t$ and the transmission $\tau$ of a single channe ${ }^{10}$ is not monotonous. Hence, the relation between the exchange coupling $J$ and the transmission $\tau$ of a channel is non-trivial.

In the absence of charging effect $\left(E_{C}=0\right)$, the transmission $\tau$ is a nonmonotonous function of $\rho t$ (where $\rho$ is the averaged density of charge in the island and in the lead) [72]:

$$
\tau=4 \frac{(\pi t \rho)^{2}}{\left[1+(\pi t \rho)^{2}\right]^{2}} \text { for } E_{C}=0
$$

A naive reasoning to obtain $G_{1 \mathrm{CK}}^{*}$ is to substitute $t$ by $J$ in this expression, and evaluate it at the 'spin' $1 \mathrm{CK}$ fixed point $J \longrightarrow \infty$. The fixed point for the 'charge' $1 \mathrm{CK}$ model derived from this naive reasoning is thus $G_{1 \mathrm{CK}}^{*}=0$.

\section{Prediction for the conductance in the tunnel limit $\tau_{1,2} \ll 1$ in the $2 \mathrm{CK}$ model}

Furusaki and Matveev have studied analytically the 2CK model. We focus on their prediction for symmetric coupling of the two channels (since the asymmetry between the channels is a relevant perturbation that will drive the system away from the 2CK fixed point [93, 2]).

The two limits treated analytically are those for $\tau_{1}=\tau_{2} \triangleq \tau \ll 1$ and $1-\tau \ll 1$. The first one is the tunnel case; the second is the strong coupling case.

\footnotetext{
${ }^{10}$ In practice realized with a QPC in the QHE regime.
} 


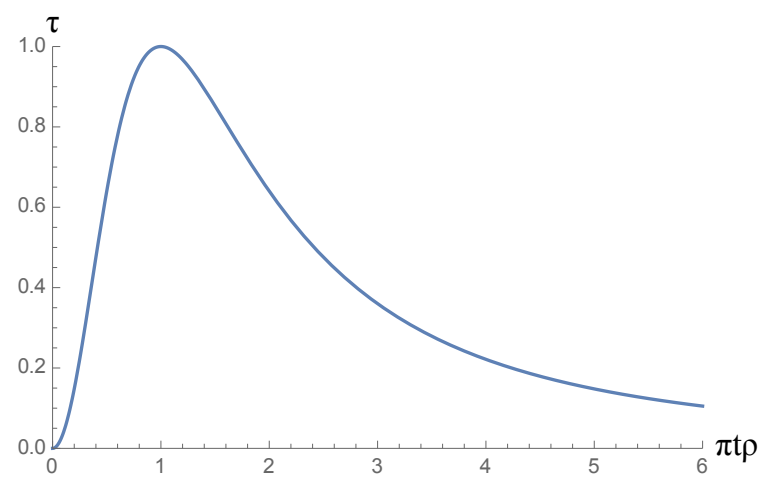

Figure 3.8: Plot of Eq. 3.7. The transmission $\tau$ tends to zero at large $t$. The maximum $\tau=1$ is reached at $t=1 /(\pi \rho)$.

In the tunnel case, both channels are first renormalized independently because the couplings are initially weak. The conductance of each channel thus initially increases logarithmically as in the $1 \mathrm{CK}$ [43]:

$$
G^{\tau \ll 1}=\frac{1}{2} \frac{\pi^{2}}{\ln ^{2}\left(T / T_{K}\right)} G_{K}
$$

with the scaling Kondo temperature:

$$
T_{K}^{\tau \ll 1} \simeq \frac{E_{C}}{k_{B}} \exp \left(\frac{-\pi^{2}}{2 \tau}\right)
$$

\section{Power law of the conductance versus temperature near the $2 \mathrm{CK}$ fixed point}

In the strong coupling regime, the quantitative expression of the conductance has been calculated [43]. We already used this expression for the charge quantization in Chapter 2. Here we just focus on the correction proportional to $T$ that vanishes at low temperature at degeneracy (when approaching the $2 \mathrm{CK}$ fixed point):

$$
G_{2 \mathrm{CK}}^{\delta \tau \ll 1}=G_{K} \times\left(1-\frac{T}{2 T_{K_{2 \mathrm{CK}}}^{\delta \tau \ll 1}(\tau)}\right)
$$

where we have put all the parameters and constants in a coefficient that defines a scaling Kondo temperature in the strong coupling regime $\delta \tau \triangleq|1-\tau| \ll 1$ :

$$
k_{B} T_{K_{2 \mathrm{CK}}}^{\delta \tau \ll 1}(\tau)=\frac{2 E_{C}}{\pi^{3} \gamma \delta \tau}
$$

All the expressions of scaling temperatures we have given for the $2 \mathrm{CK}$ model increase with $\tau$. Indeed, a higher $\tau$ set the initial value closer to the fixed point 
(which is reached at the strong coupling limit $\tau=1$ ), the effective temperature $T / T_{K}(\tau)$ should then be lower.

The strong coupling expression $T_{K_{2 \mathrm{CK}}}^{\delta \tau \ll 1}$ diverges at $\tau \sim 1$. Setting $\tau$ close to this critical value gives access to Kondo temperatures that are even higher than our cutoff energy $E_{C}$ ! Note that the Kondo model applies even if $T_{K}>E_{C}$, since the only practical requirement is $T \ll E_{C}$.

\section{Power law of the conductance versus temperature near the $3 \mathrm{CK}$ fixed point}

Using a perturbation theory and a dimensional analysis, Simon and Mora have evaluated the deviation from the fixed point $\Delta G \triangleq\left|G-G^{*}\right|$ for $N>2$ number of channels ${ }^{11}$ at degeneracy $\delta V_{g}=0$ with symmetric couplings $\tau$ and at low temperature [112]:

$$
\Delta G=c_{t}\left(\frac{T}{T_{K}^{\delta \tau \ll 1}}\right)^{\Delta}
$$

where $c_{t}$ is a constant of order of 1 and $\Delta \triangleq \frac{2}{2+N}$ is related to the dimension $d_{O_{1}}=1+\Delta$ of the leading irrelevant operator $O_{1}$.

Assuming $T_{K}^{\delta \tau \ll 1}$ diverges at a critical value $\tau_{C}$, our collaborators have been able to evaluate $T_{K}(\tau)$ near $\tau_{C}$. They have found that the conductance should follow a scaling law as [3, 88]: $G\left(\left(T / T_{K}\right)^{\Delta}\right)=G\left(A \delta \tau\left(k_{B} T / E_{C}\right)^{\Delta}\right)$ where $A$ is numerical factor of order 1 . Hence the scaling Kondo temperature near the fixed point (for all $N>2$ ) is:

$$
k_{B} T_{K}^{\delta \tau \ll 1} \simeq \frac{E_{C}}{\delta \tau^{1 / \Delta}}
$$

where $\delta \tau \triangleq\left|\tau-\tau_{C}\right|$. We summarize the theoretical laws that we will use in Table 3.3 .

\begin{tabular}{|c|c|c|c|}
\hline$N$ & $\Delta G$ & $k_{B} T_{K}^{\delta \tau \ll 1}$ & Reference \\
\hline 2 & $\propto T / T_{K_{2 C K} \delta \tau 1}^{\delta / 5}$ & $\sim E_{C} / \delta \tau$ & {$[43]$} \\
3 & $\propto\left(T / T_{K_{3 C K} \tau \ll 1}^{\delta \tau}\right)^{2 / 5}$ & $\sim E_{C} / \delta \tau^{5 / 2}$ & {$[112]$} \\
\hline
\end{tabular}

Table 3.3: Power laws for the conductance near the $N$-channel 'charge' Kondo fixed point

${ }^{11}$ The case $N=2$ is special because the fixed point is reached at an extremal value of $\tau(\rho J)=1$ (see Fig. 3.8. 


\section{Universal scaling}

One of the most important features of the original Kondo model is that a unique parameter $T_{K}$ contains all the microscopic details of the system (energy cutoff $D$, charging energy $E_{C}$, transmission $\tau$ ). It means that the temperature can be rescaled in $T / T_{K}$ for any observable to obtain a universal curve. Different experiments realized under different conditions should all collapse on this universal curve after rescaling (whatever the microscopic details).

However, a given observable should renormalize enough to escape a transitory non-universal regime before reaching the universal one $\left(T \ll E_{C}, D\right)$. Actually, if one wants to get the full universal curve, one should start with a very low $T_{K}$ (compared to the cutoff energy $T_{K} \ll D$ ) [92, 85]. Note that it does not mean that one cannot reach the universal limit starting with a large $T_{K}$. The universal limit is actually reached as soon as $T \ll D$ irrespectively of the value of $T_{K}$. The only disadvantage of starting with a large $T_{K}>D$ is that only a fraction of the universal curve ( $T \ll D<T_{K}$, close to the fixed point) will be accessible. For instance, if $T_{K} \sim D$ : the upper half of the curve $T / T_{K} \ll 1$ will be universal because $T \ll D$; whereas the the lower half $T / T_{K} \gg 1$ will be sensitive to the cutoff $D$ and thus non-universal.
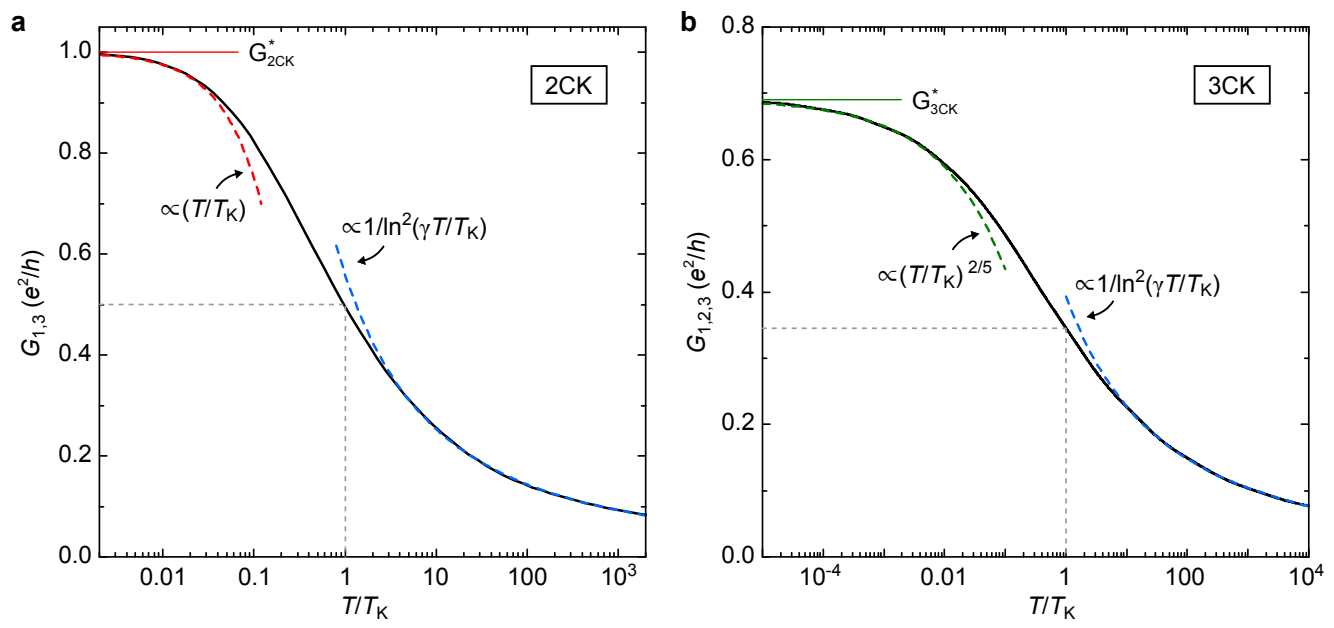

Figure 3.9: Universal curves of the conductance for the two- and threechannel 'charge' Kondo model $[\mathbf{8 5}, \mathbf{5 1}]$. The conductance is plotted versus $T / T_{K}$ in a log scale. The conductance of the fixed points at indicated with colored solid lines (red for $2 \mathrm{CK}$ and green for $3 \mathrm{CK}$ ). The Kondo temperature $T_{K}$ is defined such that $G\left(T=T_{K}\right)=G^{*} / 2$ (see grey dashed lines). The colored dashed lines are the analytical prediction in the tunnel regime (blue) and close to the $2 \mathrm{CK}$ (red dashed) and 3CK (green dashed) fixed point. These lines are horizontally shifted ( $\gamma$ is a numerical factor) to fit to the universal curve. 
Following our publication of a non-universal experimental curve of the conductance for the 'charge' 2CK model [52], Mitchell and co-workers have modified their powerful interleaved NRG code [86, 113] to implement the 'charge' Kondo model. They have been able to start with a small $T_{K}$ (that corresponds to the tunnel limit, far from the fixed point) and decrease the temperature over many orders of magnitude to get numerically the full universal curve of the conductance [85]. This curve is reproduced in Fig. 3.9 a. Mitchell has also calculated the universal conductance curve for the $3 \mathrm{CK}$ model, but only when approaching the intermediate fixed point from below, see Fig. 3.9p [51].

This universal conductance curve can be compared to the analytical predictions close to the fixed point $T \ll T_{K}$ (Table 3.3) and also in the tunnel regime $T \gg T_{K}$ Eq. (3.8) (where the channels are renormalized independently).

\subsubsection{Experimental implementation}

One may wonder: "But why such hopeful proposal established in the early 90's has never been implemented in practice?". In this subsection, we will see why it is not obvious to design a sample that realizes this model. Then we will explain how we managed to solve this problem.

\section{Contradicting requirements}

On page 55, we have listed three necessary hypotheses needed to implement the 'charge' Kondo model. In 2000, Zarárand and co-workers have used a renormalization group approach to evaluate the possibility to observe $2 \mathrm{CK}$ behaviors in a SET [136]. In their conclusion, the authors explore two scenarios of experimental implementations to observe $2 \mathrm{CK}$ in practice: (i) a metallic island (ii) a $2 \mathrm{D}$ island made in a semiconductor.

In the scenario (ii), a good control of the number of channels is possible. One should try to make the smallest possible island to increase $E_{C}$ because the Kondo mapping only applies at $k_{B} T \ll E_{C}$. However, the mean level spacing $\delta E$ in the 2D island will then not be negligible compared to $k_{B} T$. But having $\delta E \ll k_{B} T \ll E_{C}$ is mandatory to observe the $2 \mathrm{CK}$ effect: in the scenario (ii) $2 \mathrm{CK}$ will not develop because of the $2 \mathrm{D}$ geometry of the island and the small effective mass of the electrons in the 2DEG (for numbers, see Appendix B.2.1).

The scenario (i) does not have any problem concerning density of state requirement since the island is $3 \mathrm{D}$ and metallic. Moreover large $E_{C}$ are available, and one can easily fulfill $\delta E \ll k_{B} T \ll E_{C}$. The problem in this case is to connect the island with a few symmetric conduction channels. The authors of ref. [136] suggest to use an atomic contact between a metallic droplet and the tip of an 
STM. However this implementation might not be stable enough to observe $2 \mathrm{CK}$ behaviors with a good precision.

\section{The solution: a hybrid metal-semiconductor nanostructure}

We have been able to conciliate both the requirement of a negligible level spacing and the one of a good control of the number of channel thanks to a hybrid nanostructure. The island of our sample is metallic while the junctions are made in a semiconductor (with QPCs). This nanofabricated structure has all the advantages, and it is therefore the ideal sample to explore multi-channel Kondo physics. An important point when fabricating this hybrid nanostructure is to make a perfect connection between the metallic and the semiconducting part, since otherwise the description of the sample would have to take into account a residual reflection. How well this is achieved and other details about the metallic island are given in Appendix B.2.

Our sample is used in the QHE regime (that breaks the spin degeneracy). It contains three QPCs facing the metallic island; we can then access the two- and threechannel Kondo regimes and observe them through conductance measurements.

\subsection{Observation of the multi-channel 'charge' Kondo effect}

We will now present our experimental observation of the multi-channel Kondo effect using the 'charge' implementation. In particular the experimental data will be compared to the predictions given previously.

This section is divided in two parts, the first one shows the flow of the conductance of the device towards the predicted 2- and 3-CK fixed points (depending on the configuration of the QPCs). The second part focuses on scaling and universality.

The Appendix E explains the experimental procedures to get the data shown in this section while avoiding experimental artifacts. Its reading is not required to understand this section.

\subsubsection{Kondo fixed points}

We will start this subsection by showing Coulomb blockade peaks renormalized by Kondo effect. We will see that the value at the charge degeneracy $\delta V_{g}=0$ will tend to the predicted fixed point. These first data already demonstrate that our sample realizes the multi-channel 'charge' Kondo model. Then we will focus on 
$\delta V_{g}=0$ to observe the flow of the conductance towards the fixed point as we lower the temperature.

\section{Coulomb peak renormalization by Kondo effect}

The Coulomb blockade oscillations of the conductance observed in our sample are renormalized due the 'charge' Kondo effect. The gate voltage $V_{g}$ acts as an effective magnetic field that destroys the effect, which is thus maximal at charge degeneracy $\delta V_{g}=0$ (zero effective magnetic field). Figure 3.10 shows Coulomb peaks in the $2 \mathrm{CK}$ and $3 \mathrm{CK}$ regimes for two temperatures and two transmissions. As predicted by theory, we observe a renormalization towards the predicted fixed points as the temperature is lowered.
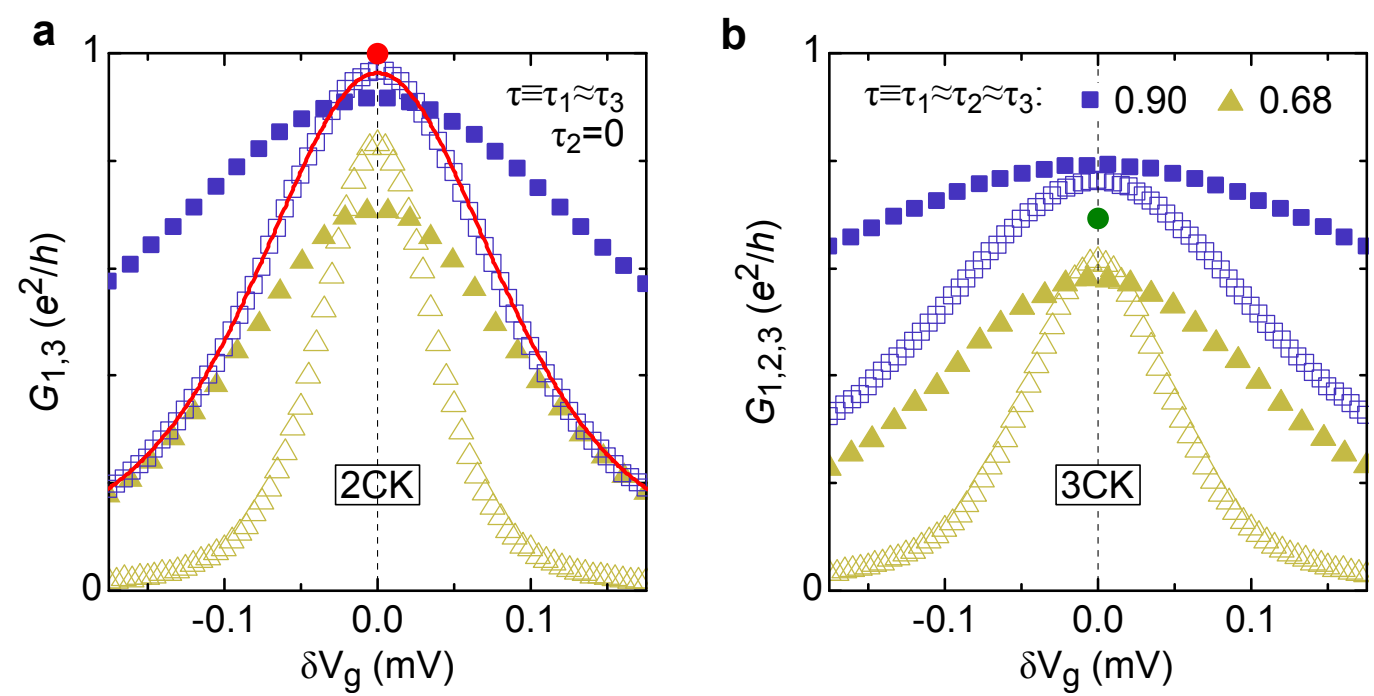

Figure 3.10: Renormalization of Coulomb blockade conductance peaks by the 2- and 3-channel 'charge' Kondo effect. Coulomb peaks of conductance are plotted as a function of the plunger gate voltage $V_{g}$ for different temperatures ( $T \approx 7.9 \mathrm{mK}$ for the open symbols and $29 \mathrm{mK}$ for the filled ones) and 2 different symmetric transmissions configurations ( $\tau \approx 0.68$ for the triangles and $\tau \approx 0.90$ for the squares) in the $2 \mathrm{CK}(\mathbf{a})$ and $3 \mathrm{CK}(\mathbf{b})$ regimes. a, The conductance $G_{1,3}\left(\delta V_{g}=\right.$ 0 ) flows towards the 'charge' $2 \mathrm{CK}$ fixed point $G_{2 \mathrm{CK}}^{*}=G_{K}$ when lowering the temperature. The red line is the quantitative theoretical prediction of the 'charge' $2 \mathrm{CK}$ model plotted for the independently measured parameters. b, In the $3 \mathrm{CK}$ case, the individual symmetric conductances at degeneracy $G_{1,2,3}\left(\delta V_{g}=0\right)$ flows to the 3CK fixed point $G_{3 \mathrm{CK}}^{*} \approx 0.691 G_{K}$.

In the $2 \mathrm{CK}$ case the conductance at degeneracy increases with the temperature for both transmissions. Away from degeneracy $\delta V_{g} \neq 0$, we observe however an opposite behavior. This decrease is simply explained by the fact that the Kondo 
effect is destroyed in this region. The conductance is then described by DCB which blocks the current at low energies (low temperature here). This explains that for a given transmission, the curves at two different temperatures are crossing each other.

The situation is different in the $3 \mathrm{CK}$ case where the fixed point is at an intermediate value between 0 and $G_{K}$. At $\delta V_{g}=0$, when we lower the temperature (passing from filled symbols to open ones), the conductance either increases (triangles) or decreases (squares) depending on the initial position with respect to $G_{3 \mathrm{CK}}^{*}$ (green disk). This signal is quite strong: Below this fixed point (triangle symbols) the traces at two different temperatures are crossing, but above the fixed point (square symbols) they are not.

From a very qualitative point of view, this figure provides a first indication of multi-channel Kondo effect. Moreover, the red line in the $2 \mathrm{CK}$ figure shows a plot of the theoretical prediction Eq. $[2.9]$ without any 12 fitting parameter. The transmissions $\tau_{1} \approx 0.891$ and $\tau_{3} \approx 0.901$ (see Fig. B.6, for the characterization of these values), the temperature $T=(7.90 \pm 0.06) \mathrm{mK}$ and the charging energy $E_{C}=$ $(25.8 \pm 0.5) \mu \mathrm{eV}$ have all been measured independently to get this quantitative comparison.

\section{Renormalization of the conductance in the $2 \mathrm{CK}$ tunnel regime $\tau \ll 1$}

Although in the tunnel regime $\tau \ll 1$, the Kondo temperature is very small $T \gg T_{K}$, the Coulomb peak is subject to a small renormalization that slowly (logarithmically) vanishes at large temperatures (see Eq. (3.8)). We have observed [52] that a tunnel peak can be well fitted with the 'orthodox' Coulomb blockade theory (Eq. (C.13) with a free amplitude which is in agreement with Eq. (3.8).

The full Kondo renormalization holds only close enough to the degeneracy $\Delta E \leqslant k_{B} T$ [43], where $\Delta E \triangleq 2 E_{C} \delta V_{g} / \Delta V_{g}$ ( $\Delta V_{g}$ is the period of the Coulomb blockade oscillations) measures the level splitting between the two charge states of the island (which are degenerate at $\Delta E=0$ ). Consequently, a Kondo renormalized tunnel Coulomb peak should be slightly narrower than a usual tunnel Coulomb peak.

This has been verified by the NRG calculation of Mitchell and co-workers. In their figure (reproduced in Fig. 3.11), they have compared their calculations (blue line) to our data (symbols). They have also plotted an 'orthodox' peak renormalized to have the same height (and which is thus broader). We see a slight difference comparable to experimental accuracy.

${ }^{12} \mathrm{We}$ have only adjusted the position of the maximum of the peak at $\delta V_{g}=0$. 


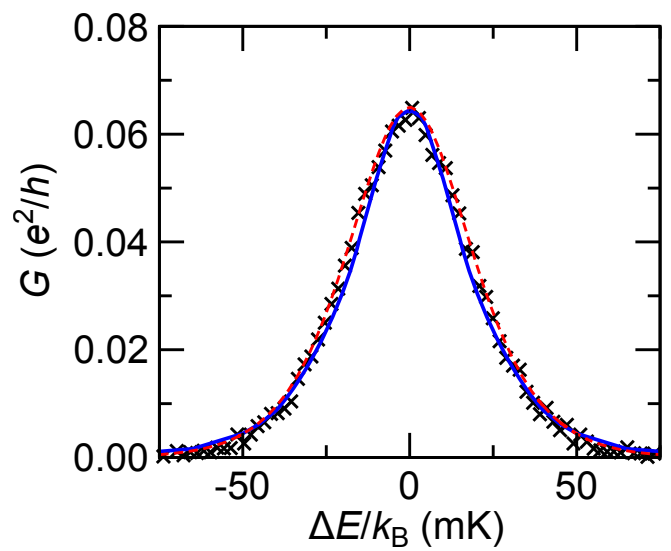

Figure 3.11: Renormalization of a 2CK tunnel Coulomb blockade conductance peak [85]. Our 2CK experimental data (symbols) [52] are compared to NRG calculations (blue solid line). The standard 'orthodox' theory (red dashed line) is vertically rescaled to have the right height.

\section{2- and 3-CK 'charge' Kondo fixed point}

Let us now focus on the points where the Kondo effect is not destroyed by energy level splitting: $\delta V_{g}=0$. To observe the renormalization towards the fixed points, we will look at the temperature dependence of the symmetric individual conductances. This convergence at low temperature is shown in Fig. 3.12 for both $2 \mathrm{CK}$ and $3 \mathrm{CK}$.

In the $3 \mathrm{CK}$ regime, the conductance flows to a non-trivial universal value $G_{3 \mathrm{CK}}^{*} / G_{K}=2 \sin ^{2}(\pi / 5)=(3-\varphi) / 2 \approx 0.691$, where $\varphi=(1+\sqrt{5}) / 2$ is the golden ratio. The accuracy of this observation is visible in the Fig. 3.13 discussed below. This is the first experimental demonstration of a Kondo coupling strength reaching a universal intermediate fixed point.

\subsubsection{Kondo scaling}

The experimental data shown in Fig. 3.12 can be compared to two theoretical predictions. The first part of this subsection focuses on the convergence towards the fixed points and checks a scaling to fit to the theoretically predicted power laws. The second one compares the experimental data to the full universal curve of conductance obtained by NRG.

\section{Power law and scaling in the vicinity of the fixed points}

In the vicinity of the Kondo fixed points, the conductance is predicted to scale as a power law $\Delta G \propto\left(T / T_{K}^{\delta \tau \ll 1}\right)^{\gamma}$, where $\Delta G$ is the distance between the conductance 

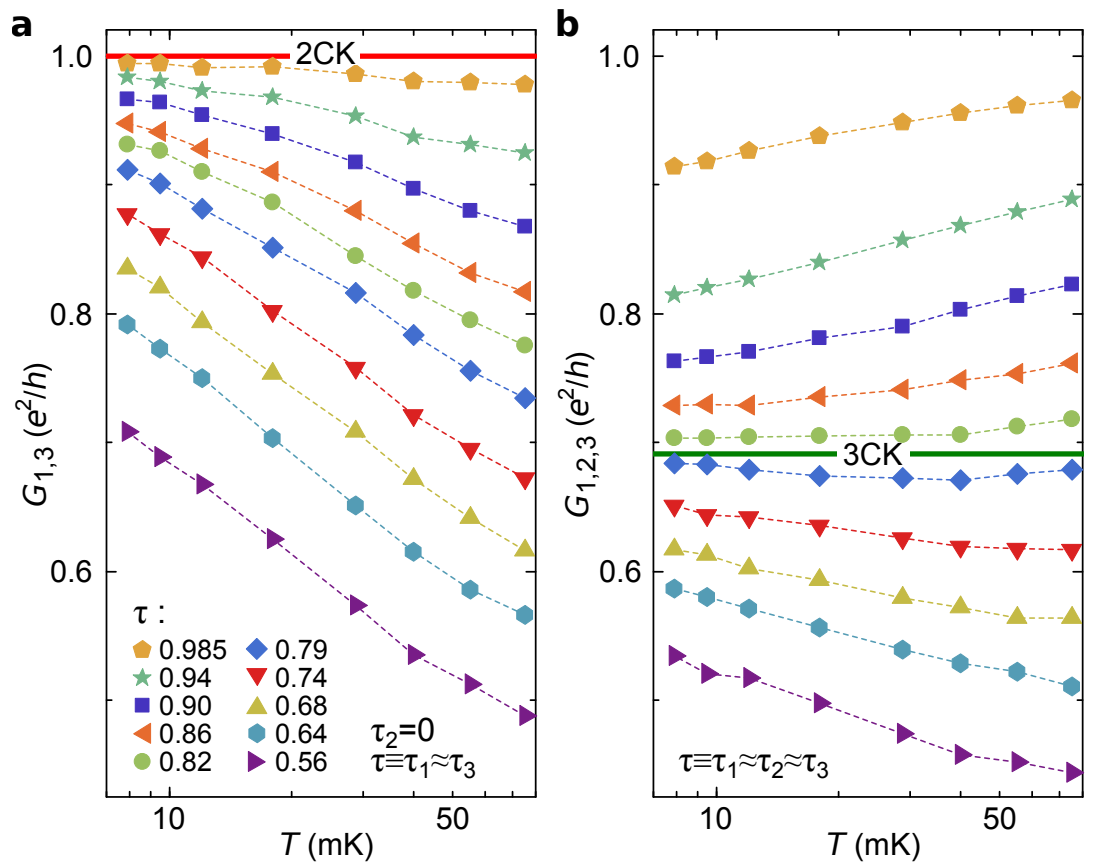

Figure 3.12: Renormalization towards the two- and three-channel 'charge' Kondo fixed point. For the same set of transmissions $\tau$, the individual conductances at degeneracy $\delta V_{g}=0$ are plotted versus the temperature $T \approx\{7.9,9.5$, $12,18,29,40,55,75\} \mathrm{mK}$ in log-scale. a, The conductance flows towards the $2 \mathrm{CK}$ fixed point (red thick line) for all the transmissions in the case of two symmetric channels $\left(\tau_{1} \approx \tau_{3}\right)$. b, With three symmetric channels, it flows to $G_{3 \mathrm{CK}}^{*}$ (green thick line) when lowering the temperature.

$G$ and the predicted fixed point $G^{*}$, and $\gamma$ is also given by the theory $\left(\gamma_{2 \mathrm{CK}}=1\right.$ and $\left.\gamma_{3 \mathrm{CK}}=2 / 5\right)$. Note that, the scaling Kondo temperature is defined up to a fixed factor (see Table 3.3).

In order to explore this scaling, we plot in Fig. 3.13 the expected power law (solid straight lines), and choose $T_{K}^{\delta \tau \ll 1}$ so that the experimental point at the lowest temperature ( $T \approx 7.9 \mathrm{mK}$ ) matches the theoretical power law. We observe consistency between experimental data and theoretical power laws. We also observe that the data closely approach the predicted fixed point $\left(\Delta G<0.01 e^{2} / h\right.$ for both $2 \mathrm{CK}$ and $3 \mathrm{CK})$.

The corresponding Kondo temperatures $T_{K}^{\delta \tau \ll 1}(\tau)$ are shown as symbols in the insets of Fig. 3.13 and compared with the appropriate theoretical predictions (which are vertically shifted since the $T_{K}^{\delta \tau \ll 1}$ are defined up to a prefactor). We observe that $T_{K}$ diverges near a critical value of transmission $\tau_{C}$. Indeed, one can get arbitrary large scaling Kondo temperatures $T_{K}$ by setting the transmission such that after an initial Coulomb renormalization, the conductances are closer to the 


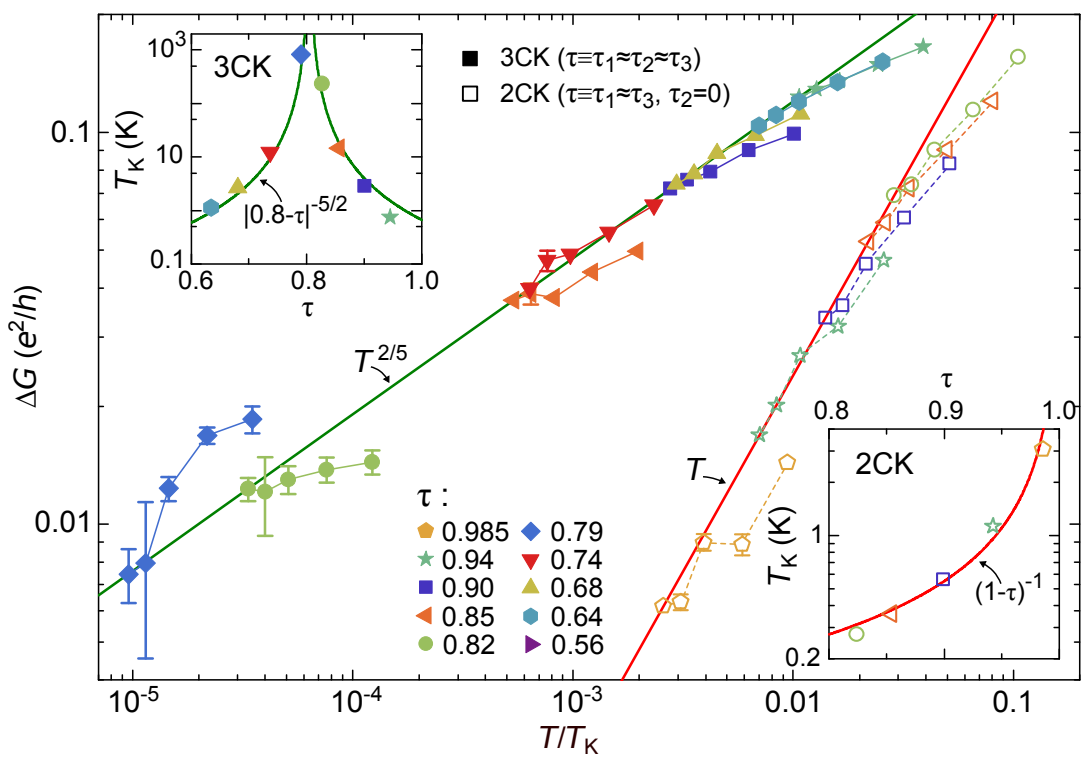

Figure 3.13: Scaling of the conductance near the 2- and 3-CK fixed points. The distance $\Delta G \triangleq\left|G-G^{*}\right|$ of the conductance from the fixed point is plotted versus a rescaled temperature. Each transmission corresponds to a symbol (solid for $3 \mathrm{CK}$ and open for $2 \mathrm{CK}$ ). By construction, the lowest temperature point of each set of symbol is on the theoretical law (solid lines). The $T_{K}^{\delta \tau \ll 1}(\tau)$ used for this scaling are given in the insets and compared with the theory (solid lines).

fixed point. For the $2 \mathrm{CK}$ case, the predicted critical transmission is $\tau_{C_{2 \mathrm{CK}}}=1$ (there is no Coulomb renormalization for this integer value). In the $3 \mathrm{CK}$ case, we find it close to $\tau_{C_{3 \mathrm{CK}}} \approx 0.8$ (which is different from $G_{3 \mathrm{CK}}^{*} / G_{K} \approx 0.691$ because of the Coulomb interaction ${ }^{13}$ ). We see that both $\Delta G$ and $T_{K}^{\delta \tau \ll 1}(\tau)$ are in a good agreement with the non-Fermi liquid power laws of the two- and three-channel 'charge' Kondo effect.

\section{Comparison with a universal scaling}

The power law of the conductance versus temperature and its scaling in the vicinity of the fixed point are actually contained in the full universal scaling curves displayed in Fig. 3.9. We can directly compare our last experimental data to these exact NRG calculations. In Fig. 3.14, we rescale the temperature in $T / T_{K}(\tau)$. Each set of data for a fixed $\tau$ is adjusted to match the point at the lowest temperature $T \approx 7.9 \mathrm{mK}$ with the theoretical universal curve. The first remark is that the remaining points $(T>7.9 \mathrm{mK})$ are following the universal prediction well. We notice deviations at large temperature, but this is due to the finite $E_{C}$ of our sample. We find that

\footnotetext{
${ }^{13}$ This initial renormalization occurs at energies of order of $E_{C}$.
} 
for $2 \mathrm{CK}$, the three lowest temperatures $(T<12.0 \mathrm{mK})$ are in the universal regime (for all $\tau$ ). The $3 \mathrm{CK}$ universal curve is smoother than the 2CK (compare both $T / T_{K}$ ranges), this may explain why the four lowest temperature data matches the universal 3CK scaling curve.
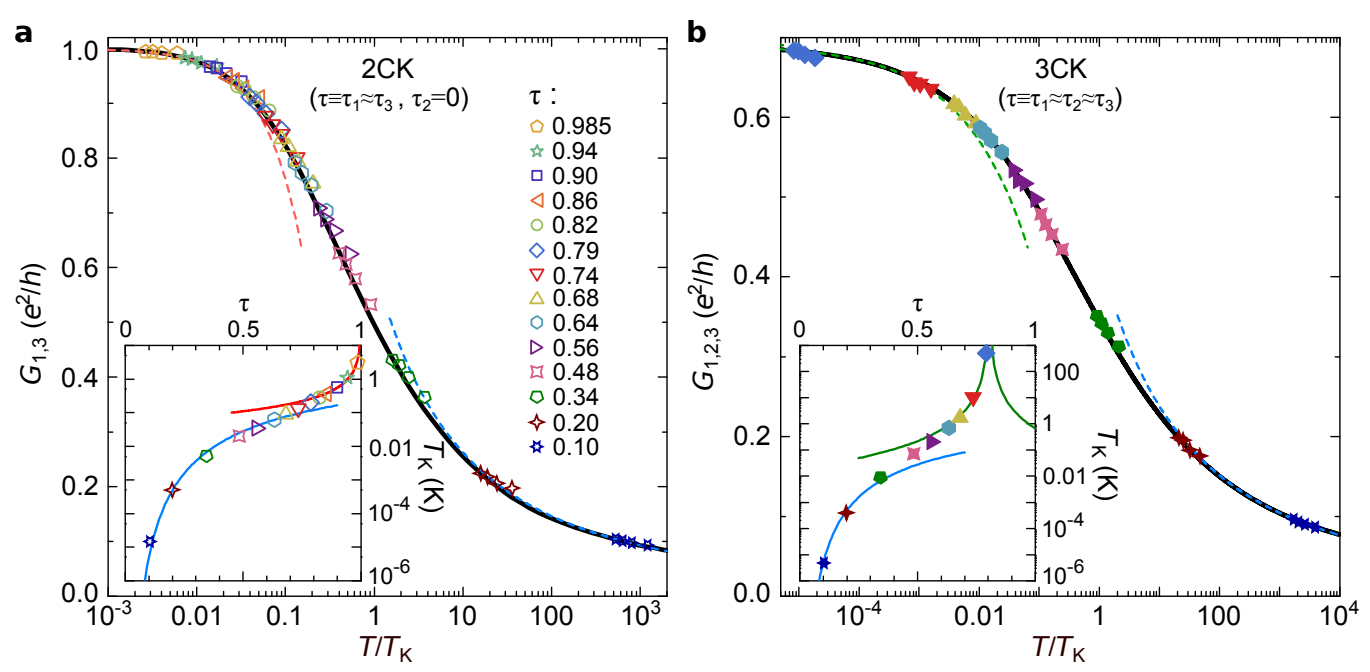

Figure 3.14: Comparison of the experimental data to the universal curves of the conductance for the 2- and 3-channel 'charge' Kondo model. Here, experimental data are displayed up to $T=29 \mathrm{mK}$ and some additional transmissions are shown compared to Fig. 3.12 Each set point at $\tau$ fixed is shifted in the semi-log representation so that the lowest temperature point matches the theoretical curve (solid black line). This defines a scaling temperatures $T_{K}(\tau)$ that are plotted in the insets for both the $2 \mathrm{CK}(\mathbf{a})$ and $3 \mathrm{CK}(\mathbf{b})$ configurations. The lines in each graph are the theoretical prediction shown in Fig. 3.9. In the insets: the blue lines correspond to the theoretical prediction in the tunnel regime; the red and green lines are the predictions for $T_{K}^{\delta \tau \ll 1}$ close to the $2 \mathrm{CK}$ and $3 \mathrm{CK}$ fixed points respectively.

We note that the deviations that develop at larger temperatures are all in the same direction. These are not experimental artifacts in the sense that our data are still modeled by the 'charge' Kondo Hamiltonian, but we are sensitive to the finite $E_{C}$. Mitchell and co-workers [85] have reproduced our experimental data with their numerical calculations by taking into account the effect of the cutoff energy. This shows that our device fully implement the 'charge' Kondo model, also beyond the universal regime where all the microscopic parameters $\left(\tau, E_{C}, D\right)$ can be encapsulated in $T_{K}$.

The scaling Kondo temperature we have used to match the first point on the universal curve also contains information: we can compare it to the theoretical expressions of $T_{K}(\tau)$. This is shown in the insets of the figure where one can see that the data near the critical transmission $\left(\tau_{C_{2 C K}}=1\right.$ and $\left.\tau_{C_{3 C K}} \approx 0.8\right)$ and the 
scaling behavior predicted for $T_{K}^{\delta \tau \ll 1}(\tau)$ (red line for the $3 \mathrm{CK}$ and green line for the $3 \mathrm{CK}$ ) are in good agreement. The theoretical lines have been vertically shifted to fit to the data at $\delta \tau \ll 1$ to account for the ill-defined prefactor of $T_{K}$. We have also plotted $T_{K}^{\tau \ll 1}$ (in blue solid), to compare with the data close to the tunnel limit $\tau \ll 1$.

\subsection{Conclusion}

The first unambiguous observation of the two-channel Kondo effect has been done in 2007 by Potok and co-workers [101]. Here we have reported an observation of both two- and three-channel Kondo effect using the 'charge' implementation proposed by Matveev in 1991 [79, 48].

In this implementation, the 'charge' degrees of freedom of a metallic island play the role of a pseudo-spin. We have conciliated the conflicting requirements on the size of the island and its density of state by using a hybrid metal-semiconductor design. Remarkably, the degenerate quantum impurity used in the present Kondo effect study is constituted of large number of electrons: it involves macroscopic quantum charge states!

This realization of the 'charge' Kondo effect accurately implements the model [85]. We have observed a quantitative agreement between the measured conductance and the theoretical prediction without any fitting parameter. We have also compared our experimental data to the universal curve of conductance for both the two- and three-channel Kondo effect. At least our three lowest temperature data, for $T \leq 12 \mathrm{mK}$, lie in the universal regime; whereas non-universal behaviors appear at larger $T$ due to the finite charging energy $E_{C}$. Hence, when lowering the temperature, we observe a crossover from a non-universal to the universal regime.

The observed non-Fermi liquid power laws for the conductance versus temperature are consistent with the predicted theoretical laws for both 2- and 3-CK. Note that it is believed that a localized Majorana fermion emerges at the $2 \mathrm{CK}$ fixed point. In this chapter, non-Fermi liquid scalings have been explored by tuning the sample right to the quantum critical points. However, a rich physics is contained in the crossover from criticality (either by breaking the channel symmetry $\Delta \tau \neq 0$ or detuning from the charge degeneracy $\delta V_{g} \neq 0$ ), and this will be the topic of the next chapter. 


\section{Chapter 4}

\section{Quantum phase transition in multi-channel Kondo systems}

Quantum criticality accounts for the unconventional physics that develops in the vicinity of the critical point of a second-order quantum phase transition [107]. It is characterized by the power law divergence of the correlation length $\xi$ as a non-thermal parameter $g$ approaches a critical value $g_{c}: \xi \propto\left|g-g_{c}\right|^{-v}$, where $v$ is called a critical exponent. The concept of quantum criticality provides a powerful universal framework to describe some of the most fascinating strongly correlated electrons phenomena, including heavy fermions [44] or high- $T_{C}$ superconductivity [59].

Although tunable nanostructures would provide ideal systems for the quantitative experimental exploration of quantum criticality, only few examples exhibit clear signatures of second-order QPTs [81, 82, 60]. In this chapter, we will show that the 'charge' implementation of the multi-channel Kondo model provides an outstanding testbed for the quantum critical physics.

We will begin with an introduction to quantum criticality. Then we present the theoretical predictions specific to the multi-channel Kondo effect. And finally we show our experimental observation of quantum criticality in our device using in turn the two relevant perturbations we have at our disposal (the gate voltage and the channel asymmetry).

\subsection{Quantum phase transition}

The physics associated with the transition between stable phases of matter has been extensively studied both theoretically and experimentally. The description of second-order phase transitions require powerful many-body techniques among which the renormalization group has a central place. Rather than being a pure 
mathematical trick, this technique has a profound physical consequence, namely universality. This field of research concerns systems as various as black holes and strongly correlated materials [107].

In this section, we will first explain what a quantum phase transition is. Then we will focus on the second-order quantum phase transition and address the notion of quantum criticality. We will discuss an observation of quantum criticality in a tunable device based on a carbon nanotube connected to dissipative leads [81, 82].

\subsubsection{What is a quantum phase transition}

\section{Definition and classification}

In contrast to classical phase transitions that occur at finite temperature, and where only thermal fluctuations have to be considered; quantum phase transitions are driven by a non-thermal parameter $g$ and they occur at zero-temperature $T=0$ where only quantum fluctuations exist.

A quantum 1 phase transition is characterized by the dependence on $g$ of various quantities, such as the correlation length $\xi(g)$, the magnetic susceptibility $\chi(g)$, the specific heat $C(g)$, or even dynamical quantities as the equilibration time $\tau(g)$.

For a first-order phase transition, some of these quantities are discontinuous. At finite temperature, there will be no critical phenomena, and the system will simply consist of a thermodynamical mixture of phases [126].

A second-order (continuous) quantum phase transition occurs at a quantum critical point $g=g_{c}$. In the vicinity of this point, the correlation length diverges as a power law: $\xi \propto\left|g-g_{c}\right|^{-v}$, where $v$ is called a critical exponent [125]. Note that for a special type of continuous transition called infinite-order transitions, the dependence on $g$ is exponential. This is the case for instance of Kosterlitz-Thouless transitions.

In this thesis, we will only discuss the quantum phase transition that present quantum criticality at finite temperature, and those are the second-order quantum phase transitions. Because of the divergence of the correlation length at the critical point, the system becomes scale-invariant. The quantum critical point is thus suitable for some theoretical approaches such as the renormalization group or the conformal field theory [24].

\section{Renormalization group theory, universality and relevant perturbations}

At the critical point, fluctuations of all wavelength have to be considered with the same weight [131]. The renormalization group theory provides a mathematical

\footnotetext{
${ }^{1}$ The classification that follows is the same for classical phase transitions, one just has to replace the non-thermal parameter $g$ by $T$.
} 
tool to treat the critical phenomena that occur in the vicinity of continuous phase transitions. This theory solves the problem iteratively. At each step, the couplings $\{K\}$ are rescaled through a transformation $\mathcal{R}[24]$ :

$$
\left\{K^{\prime}\right\}=\mathcal{R}(\{K\})
$$

In the space of all couplings' configurations, it may exist a fixed point $\left\{K^{*}\right\}$ such that $\left\{K^{*}\right\}=\mathcal{R}\left(\left\{K^{*}\right\}\right)$. This fixed point is not necessarily a quantum critical point ${ }^{2}$. Actually, the renormalization group tells nothing about the nature of the phases. Yet it describes accurately the physics near the fixed points of critical phenomena.

The same universal power laws in $t^{\gamma}$ (with $t \triangleq\left|T-T_{C}\right| / T_{C}$ for classical transition and $t \triangleq\left|g-g_{c}\right| / g_{c}$ for quantum phase transitions) can be observed for various systems [125]. Different observables (specific heat $C$, magnetic susceptibility $\chi$, relaxation time $\tau$ etc.) lead to different universal critical exponents $\alpha, \gamma, z$ etc. But few of these critical exponents are actually independent since they can be related using scaling relations that can be derived from the renormalization group [24, 125].

The fact that the same power law dependence were found for very different systems is actually well explained by the renormalization group theory. When approaching the critical point, the 'irrelevant' perturbations that depend on the microscopic details of the system vanish and the behavior becomes universal [131]. Hence many systems can be classified in a given class of universality that will be described with the same laws whatever their exact chemical composition. A class of universality can actually be defined as the basin of attraction of a fixed-point Hamiltonian [138].

\subsubsection{Quantum criticality}

As mentioned above, a second-order quantum phase transition occurs at zero temperature precisely at the quantum critical point. In practice it is unreal to reach $T=0 \mathrm{~K}$ and observe a true quantum phase transition. However, quantum criticality extends on a range of parameter that widens with temperature, up to a high temperature cutoff that depends on the underlying microscopic mechanisms.

In this subsection, we will illustrate in a phase diagram the typical parameter growing range of quantum criticality with temperature. Then we will compare this diagram with an experimental observation in a strongly correlated material. Afterwards, we will distinguish between bulk and impurity phase transitions. We will finally depict an observation of a continuous impurity quantum phase transition studied in a tunable device.

${ }^{2}$ For instance, the $J \longrightarrow \infty$ fixed point in the $1 \mathrm{CK}$ model is not a quantum critical point. 


\section{Typical phase diagram of a second-order quantum phase transition}

Let us call $g$ the non-thermal parameter that drives a continuous quantum phase transition. This transition occurs at the quantum critical point $g=g_{c}$, at zero temperature. In the vicinity of $g_{c}$ and at low temperature, the small thermal fluctuations blur the small difference between $g<g_{c}$ and $g>g_{c}$. The system then behaves as if $g=g_{c}$ and exhibits quantum criticality on a finite range of $g$ [107]. Moreover, the undetermined region widens as the temperature increases! This broadening is shown in Fig. 4.1 a. In general, as for usual quantum effects, the quantum critical behaviors vanish at high temperature ${ }^{3}$.

a

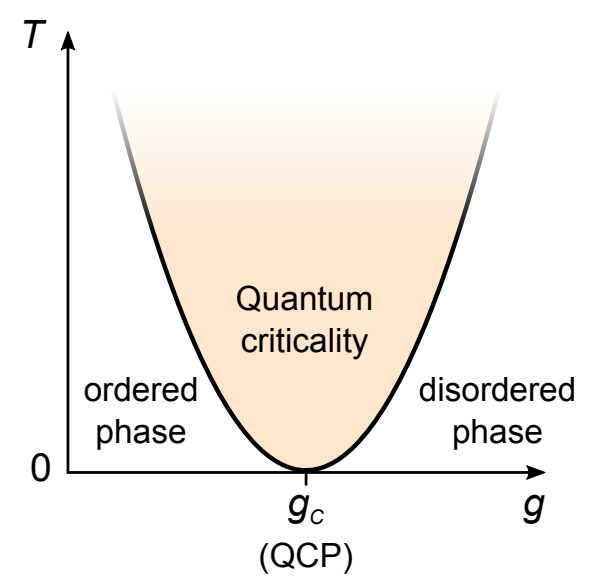

b

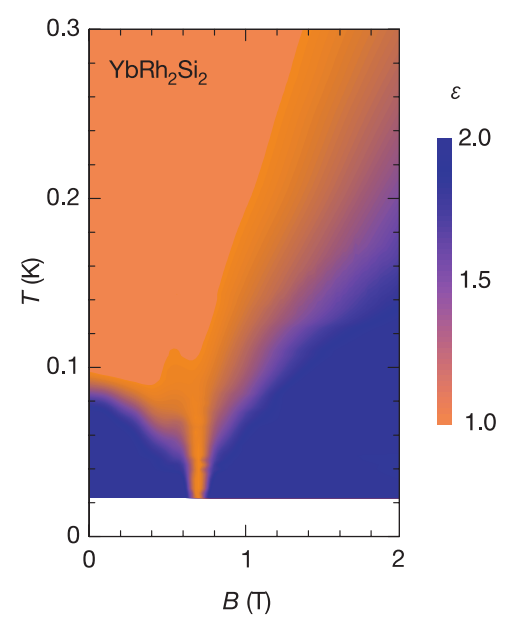

Figure 4.1: Quantum criticality a, Phase diagram near a quantum critical point $g=g_{c}$. The parameter space for quantum criticality (in orange) widens with temperature. b, Quantum critical behavior driven by a magnetic field $B$ observed in a heavy-fermion compound (reproduced from [36]). The critical exponent $\varepsilon$ of the resistivity versus temperature is non-Fermi liquid $(\varepsilon \neq 2)$ in the critical region (in orange).

\section{Quantum critical state}

The state of matter in the quantum critical region is very different from the two stable phases of the system. Above, we mentioned that the correlation length $\xi$ was diverging at the critical point of a classical phase transition, and the system was then subject to fluctuations of all wavelengths. In the quantum critical state, the system is subject to both quantum and thermal fluctuations. In electronic systems,

${ }^{3}$ Some strongly correlated materials have displayed a signature of quantum criticality up to $700 \mathrm{~K}[77]$. 
when these fluctuations diverge, they can give rise to a new type of electronic fluid of strongly correlated electrons with unconventional, non-Fermi liquid behaviors [125].

This singular state of matter is of great interest for the physicists of different fields. There is indeed a link between the conformal field theory used to describe some critical points and string theory ([59, 107] and references therein). For instance, the border of a critical region can be seen as the horizon of a black hole ([107, 28] and references therein). Quantum criticality is an active field of research, motivated in particular by the extensive work on strongly correlated materials.

\section{Quantum criticality in strongly correlated materials}

Perturbative approaches starting from free electrons fail to account for strongly correlated materials. Generally, strong correlations e.g. in the context of quantum criticality result in a breakdown of the standard Fermi liquid theory description of electronic systems.

This breakdown can be observed as non-Fermi liquid power laws for instance in the resistivity versus temperature. In standard metals, the Fermi liquid theory predicts a $T^{2}$ dependence on resistivity $\rho$. One can plot the critical exponent $\varepsilon=\mathrm{d} \ln \rho / \mathrm{d} \ln T$ versus the non-thermal parameter $g$ to observe a pattern that widens with temperature as shown in Fig. $4.1 \mathrm{p}$. Signatures of quantum criticality have been observed in heavy-fermion [75] and also in other strongly correlated materials (see [107] for examples).

In particular, a $T$-linear power law of the resistivity versus temperature is observed in the 'strange metal' phase of high- $T_{C}$ superconductors based on copper oxide [59]. Intriguingly, this critical behavior that originates from a putative quantum critical point located in the superconducting phase can span to the highest attainable temperatures [77]. To date, the theoretical description of this 'strange metal' phase is still essentially incomplete [59].

However, reaching a quantitative microscopic understanding of the superconductivity that emerges from this 'strange metal' phase at low temperature has great practical interest because of its possible technological applications. A first step is to look for systems that exhibit quantum criticality and that are easier to model and to study than the complex, real-world strongly correlated materials. A possibility is to use quantum simulators such as cold atoms in optical lattices [17] or superconducting qubits [50] to emulate bulk quantum phase transitions. An alternative route is to study 'impurity' quantum phase transitions. 


\section{'Impurity' quantum phase transition}

One can design nano-devices where some local quantum states (the quantum impurity), such as the electronic levels in quantum dots or the charge states of our metallic island, are coupled with thermodynamic baths. When a quantum phase transition occurs in such systems, the only observable that display quantum critical behaviors are those involving the quantum impurity [126].

These systems are easier to model and to treat than 'bulk' systems because of their lower dimensionality. In addition to possibly contributing to a deeper understanding of quantum criticality, 'impurity' problems can also be mapped onto higher dimensionality lattice problems. This technique is used in the dynamical mean-field theory which can treat exactly strongly correlated systems, but only in the infinite dimension limit [45].

\section{Crossover from criticality}

There are basically two ways to explore a quantum critical point. The first one has been the subject of Chapter 3, it to set $g=g_{c}$ and to lower the temperature $T$. The second one is to explore the crossover from the critical state to a stable phase (see Fig. 4.2). The power laws associated with these two experiments are sometimes related [125].

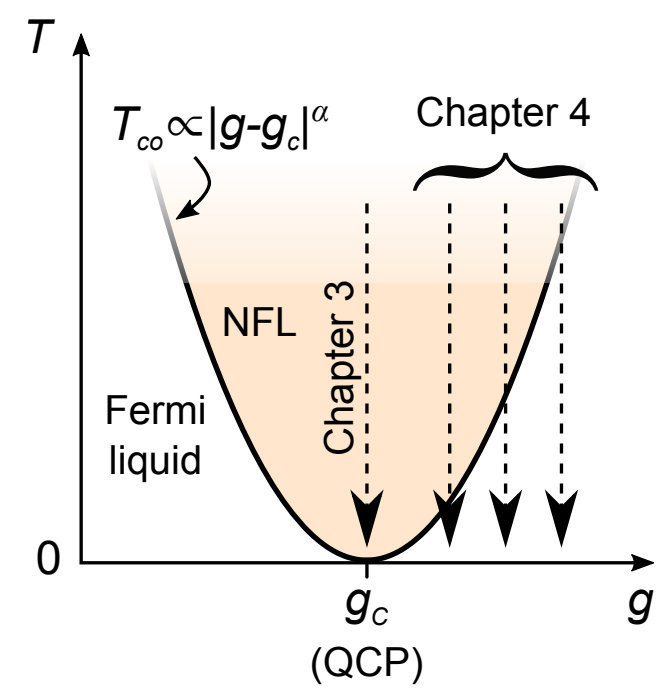

Figure 4.2: Crossover from quantum criticality. In Chapter 3, we have set the non-thermal perturbations $g=g_{c}$ and lowered the temperature to observe the power laws when flowing into quantum criticality. In the present chapter, we consider the crossover from the non-Fermi liquid (NFL) quantum critical region (in orange) to Fermi liquid. This crossover occurs on a temperature scale $T_{\mathrm{co}} \propto\left|g-g_{c}\right|^{\alpha}$. 


\section{Continuous quantum phase transitions in tunable devices}

As for the Kondo effect, tunable nano-devices could provide powerful tools for the experimental investigations of quantum criticality. However, observations of quantum criticality in tunable devices are rare. Apart from the implementation of the 2CK model using quantum dot that we have already discussed [60] in Chapter 3 , we can mention a realization by Mebrahtu and co-workers [81, 82].

They have studied the development of a second-order quantum phase transition using a carbon nanotube which acts as a quantum dot. The carbon nanotube was connected to two dissipative leads through tunnel junctions. At low temperature, the conductance drops because of dynamical Coulomb blockade, except at the QCP corresponding to symmetric contacts, where the conductance tends to the unitary limit $G_{K}=e^{2} / h$. With this device, a non-Fermi liquid power law has been observed on the conductance versus temperature when approaching the quantum critical point, and the broadening of the quantum critical area with temperature has also been studied (see Fig. 4.3).
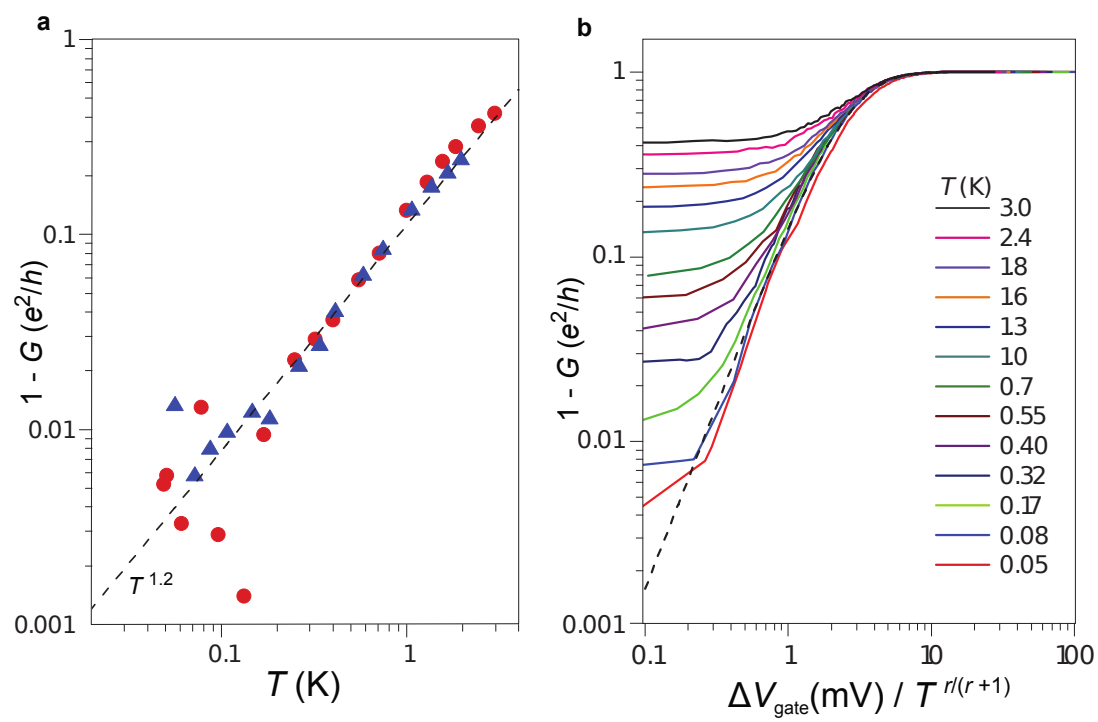

Figure 4.3: Quantum criticality observed by Mebrahtu and co-workers [82]. a, A power law $|1-G| \propto T^{1.2}$ is observed when approaching the quantum critical point. b, Crossover from criticality when breaking the symmetry using $\Delta V_{\text {gate }}$ : the larger the temperature, the wider the range where the conductance $G$ saturates close to the unitary limit. 


\subsection{Quantum phase transitions in the multi-channel Kondo model}

A flow towards a non-Fermi liquid fixed point is predicted in the overscreened multi-channel Kondo effect. However, any finite magnetic field or an asymmetry on the channels' bare couplings lead to a different fixed point at low temperature. As detailed below, this quantum phase transition is of second-order and displays non-Fermi liquid quantum critical physics.

In this section we will first consider the general multi-channel Kondo model, discuss quantum criticality and define the crossover temperature. We will also present the crossover from criticality observed by Keller and co-workers in their 'spin' $2 \mathrm{CK}$ implementation. Then we will focus on the 'charge' implementation and present the predictions for the two possible relevant perturbations (channel or pseudo-spin asymmetry).

\subsubsection{Description of the quantum phase transition}

The 2CK model has been extensively studied theoretically and exact results have been obtained. The ground state of this model involves non-Fermi liquid physics. However, the tiniest (non-zero) channel asymmetry will drive the system towards a Fermi liquid. The crossover from the strongly correlated physics to the free fermion description occurs below a characteristic crossover temperature $T_{\mathrm{co}}$ (provided the system was initially in the quantum critical regime $T_{\text {co }}<T \ll T_{K}$ ). At the end of this subsection, we will present the results of Keller and co-workers who observed this crossover when breaking the channel symmetry [60].

\section{Quantum critical point}

The non-Fermi liquid quantum critical physics of the overscreened Kondo impurity is well-established theoretically. Critical exponents of this quantum critical point have been first derived via the Bethe ansatz method [13, 117]. The CFT can be applied to the conformally invariant critical points of the multi-channel Kondo model to get exact results for the power law of the magnetic susceptibility $\chi(T)$, specific heat $C(T)$ or resistivity $\rho(T)$ versus temperature [3, 4]. Of course, numerical renormalization group can also be used to study the fixed points [20]. Because of its relative simplicity, the $2 \mathrm{CK}$ model has become a paradigmatic example for the non-Fermi liquid physics. 


\section{Crossover from criticality}

The asymmetry between the antiferromagnetic couplings (e.g. $J_{1} \neq J_{2}$ ) is a 'relevant' perturbation in the renormalization group sense (i.e. it grows under renormalization). This is illustrated in Fig. 4.4, which shows that the 2CK intermediate fixed point is unstable under a channel perturbation. This perturbation will drive the system towards a Fermi liquid at low temperature. The smaller the asymmetry $\Delta J$, the lower the crossover temperature to Fermi liquid will be.

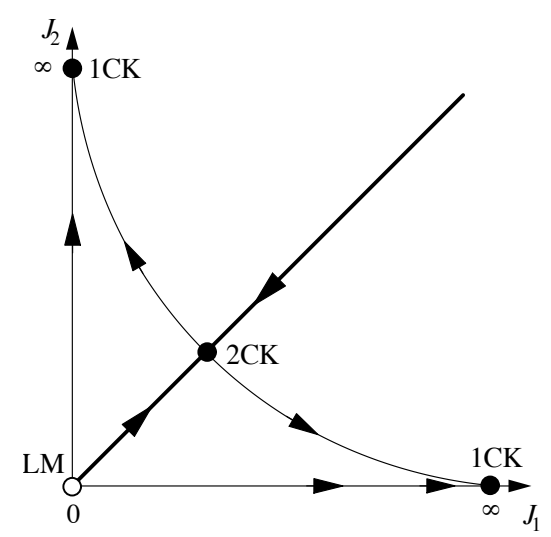

Figure 4.4: Renormalization flow diagram of the 2CK model (reproduced from [126]). The arrows are pointing towards low temperatures. This diagram shows the renormalization of the exchange coupling $J_{i}$ of each channel. One can see the finite coupling 2CK fixed point and the 1CK fixed points (at infinity). 'LM' is the local-moment fixed point of an uncoupled impurity $\left(J_{1}=J_{2}=0\right)$.

Moreover, one can destroy the Kondo effect by applying a magnetic field that will favor one of the two position of the spin $\vec{S}$ of the impurity. This will drive the system towards a Fermi liquid phase (even for symmetric channels). In the 'charge' implementation, a detuning of the voltage gate $\delta V_{g}$ corresponds to an effective magnetic field. In this chapter, we will use the energy level splitting $\Delta E \triangleq 2 E_{C} \delta V_{g} / \Delta V_{g}$ rather than $\delta V_{g}$.

Note that in the $3 \mathrm{CK}$ model, a channel anisotropy $J_{1}=J_{2}>J_{3}$ drives the system from the $3 \mathrm{CK}$ NFL fixed point to the $2 \mathrm{CK}$ NFL fixed point. Therefore, a channel anisotropy does not necessarily generate a crossover towards a FL ground state. This is in contrast with an energy level splitting $\Delta E$. We will experimentally explore both of these type of crossovers (using $\Delta J$ and $\Delta E$ separately).

\section{Crossover temperature $T_{\mathrm{co}}$}

As shown in Fig. 4.2, a power law $T_{\text {co }} \propto\left|g-g_{c}\right|^{\alpha}$ delimits the quantum critical region from the Fermi liquid phase. In the multi-channel Kondo effect, the non- 
thermal parameter $g$ can be either the channel asymmetry $\Delta J$ or the effective magnetic field $\Delta E$ (or a combination).

Cox and Zawadowski give the general expression of $T_{\text {co }}$ for a channel or magnetic field perturbation (see Section 5.1.4. in their extensive review [29]). We will only consider their result on the effective magnetic field $\Delta E$, for the $N$-channel Kondo model (with $N>1$ ):

$$
T_{\text {co }} \propto \Delta E^{\beta} \text { with } \beta=\frac{2+N}{N}
$$

For a channel symmetry perturbation, the crossover temperature is given by

$$
T_{\text {co }} \propto \Delta J^{\eta} \text { with } \eta=\frac{2+N}{2},
$$

Note that the two exponents $\beta$ and $\eta$ are equal only for $N=2$.

\section{Crossover from criticality in tunable devices}

In 2015, Keller and co-workers have observed the $\Delta J$ driven crossover from quantum criticality in a $2 \mathrm{CK}$ experiment [60]. In their 'spin' implementation, a true magnetic field, a channel asymmetry or coherent charge transfers between the reservoirs are relevant perturbations. They have used a lateral gate voltage (named $V_{\mathrm{BWT}}$ ) to break the symmetry of the channel couplings to their quantum dot. In Fig. 4.5 a, traces of the conductance versus voltage bias $\left(V_{\mathrm{SD}}\right)$ are plotted. The $V_{\mathrm{BWT}}$ value used (vertical dashed line in Fig. $4.5 \mathrm{p}$ ) is slightly away from the QCP. Therefore, $T_{\mathrm{co}}>0$ and the system is experiencing quantum criticality only at high temperatures $T>T_{\mathrm{co}}$. The deviation between the low temperature traces and the universal $2 \mathrm{CK}$ predicted behavior (solid black line) signal $T \lesssim T_{\mathrm{co}}$.

A crossover temperature scale $T_{\mathrm{co}}$ can be extracted from the fit of the conductance traces with a $2 \mathrm{CK}$ prediction. This temperature scale is shown in Fig. $4.5 \mathrm{~b}$ with respect to $V_{\mathrm{BWT}}$. In the $2 \mathrm{CK}$ model, we know from the previous paragraph that $T_{\text {co }} \propto \Delta J^{2}$. The red lines of this figure are thus fits to piecewise 4 parabolas that delimit the quantum criticality area (above the parabolas).

One may notice that the power law used in Fig. 4.5 to rescale the experimental data is $\propto \sqrt{T}$ rather than the $T$-linear power law we have observed in our 'charge' $2 \mathrm{CK}$ measurement. This predicted difference can be intuitively understood from the fact that the conductance $G$ of the $2 \mathrm{CK}$ fixed point in the 'charge' implementation is reached at the extremal value $G\left(J_{2 \mathrm{CK}}^{*}\right) \triangleq G_{2 \mathrm{CK}}^{*}=e^{2} / h$. Consequently, from a second-order development $\frac{\mathrm{d} G}{\mathrm{~d} J}\left(J=J_{2 \mathrm{CK}}^{*}\right)=0$ and one naively

\footnotetext{
${ }^{4}$ It is argued in [60] that in general, the prefactor of a critical power law as $T_{\text {co }} \propto \Delta J^{2}$ can be different on each side of a quantum critical point. The reason for such an anisotropy is unclear to us.
} 

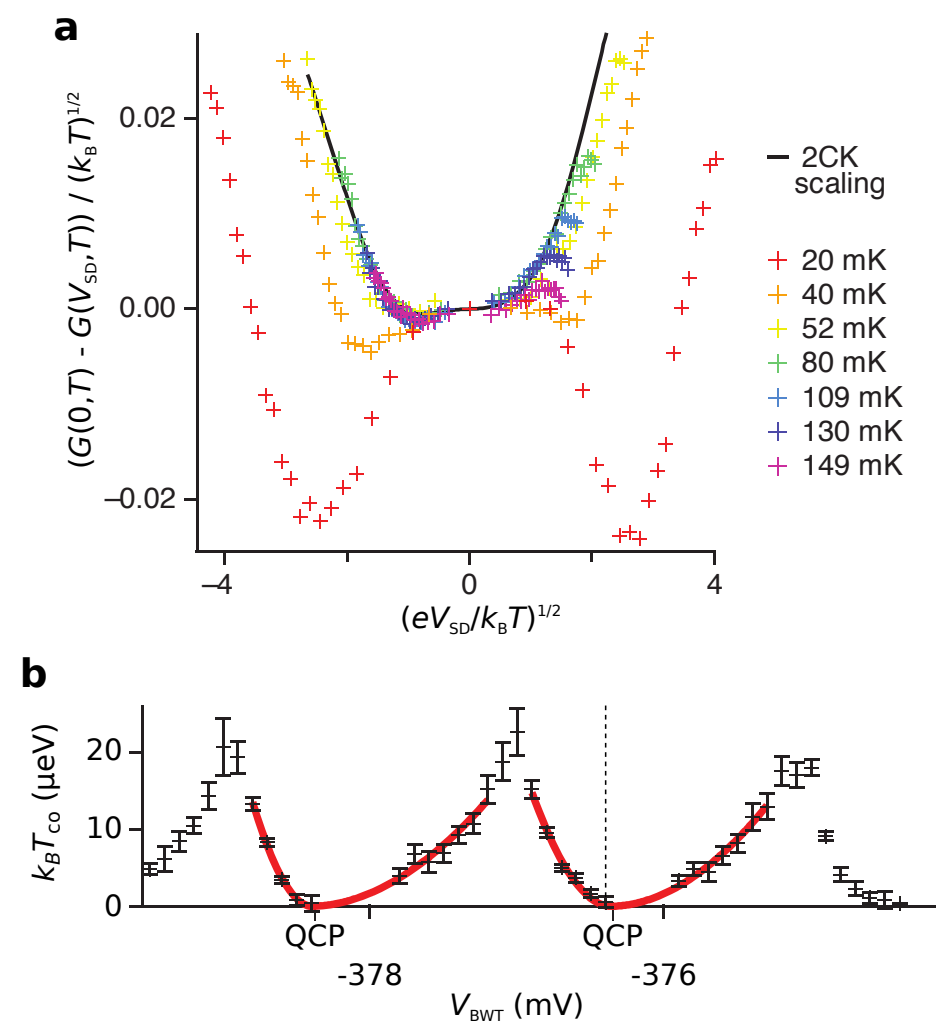

Figure 4.5: Crossover from quantum criticality observed in the 2CK experiment of Keller and co-workers [60]. a, The rescaled conductance is plotted versus the rescaled voltage bias. The high temperatures traces follow the universal behavior (black line) whereas at low temperature deviations are observed. b, A crossover temperature $T_{\text {co }}$ can be extracted from a fit of the conductance shown in a to a $2 \mathrm{CK}$ prediction. This $T_{\mathrm{co}}$ is then fitted with half parabolas (that are periodic just as the Coulomb oscillations). The vertical dashed line indicates the voltage gate $V_{\text {BWT }}$ used in $\mathbf{a}$.

expects $G\left(J\left(T \ll T_{K}\right)\right) \approx G\left(J_{2 \mathrm{CK}}^{*}+\alpha \sqrt{T}\right) \approx G_{2 \mathrm{CK}}^{*}+1 / 2 \frac{\mathrm{d}^{2} G}{\mathrm{~d} J^{2}}\left(J_{2 \mathrm{CK}}^{*}\right)(\alpha \sqrt{T})^{2}$. Thus $\left|G\left(T \ll T_{K}\right)-G_{2 \mathrm{CK}}^{*}\right| \propto T$. Note that for $N \geqslant 3$, the same standard power law versus the temperature is expected for the conductance in both the 'spin' and the 'charge' implementations.

\subsubsection{Theoretical predictions for the 'charge' Kondo model}

\section{Universality in the vicinity of the $2 \mathrm{CK}$ fixed point}

Considering the $2 \mathrm{CK}$ model is very informative since the full quantitative prediction of the $2 \mathrm{CK}$ conductance has been computed by Matveev and Furusaki near the 
2CK fixed point. They have found the following expression for the conductance along the crossover from a fully developed quantum criticality $\left(T \ll T_{K}\right)$ :

$$
\tilde{G}_{2 \mathrm{CK}}^{T \ll T_{K}}(T, \Delta \tau, \Delta E)=G_{K}\left[1-\frac{T_{\mathrm{co}}(\Delta \tau, \Delta E)}{2 \pi T} \psi^{\prime}\left(1 / 2+\frac{T_{\mathrm{co}}(\Delta \tau, \Delta E)}{2 \pi T}\right)\right]
$$

where $\psi$ is the digamma function. Quite generally, in the vicinity of a Kondo quantum critical point (i.e. once $T \ll T_{K}$ ), the only energy scale to consider is $T_{\text {co }}$ and observables can be expressed as universal functions of $T / T_{\text {co }}$ [102, 87, 85]. This expression has been derived with another analytical method by Mitchell and co-workers, starting from the opposite limit $\tau \ll 1$, and for both magnetic field and channel asymmetry perturbations [85]. For $N=2$ channels, the critical exponents associated to the two relevant perturbations are the same: $T_{\mathrm{co}} \propto \Delta \tau^{2}$ and $T_{\mathrm{co}} \propto \Delta E^{2}$; and they have the following expression for the crossover temperature [85]:

$$
T_{\mathrm{co}}=c_{1} T_{K} \Delta \tau^{2}+c_{2} \Delta E^{2} / T_{K}
$$

where $c_{1}$ and $c_{2}$ are non-universal parameters ( $T_{\mathrm{co}}$ itself is defined up to an arbitrary prefactor). Furusaki and Matveev have derived the quantitative prediction for $T_{\mathrm{co}}(\Delta \tau, \Delta E)$ from the Hamiltonian parameters $\tau, \Delta E, E_{C}$. Expending their expression to second order in $\Delta \tau \ll 1-\tau \ll 1$ and $\Delta E \ll E_{C}$, one finds [43]:

$$
T_{\mathrm{co}} \sim \frac{\pi \gamma^{2}}{4} T_{K} \Delta \tau^{2}+\frac{4}{\pi^{3}}\left(\Delta E / k_{B}\right)^{2} / T_{K}
$$

where $k_{B} T_{K} \triangleq 2 E_{C} /\left(\pi^{3} \gamma(1-\tau)\right)$ is defined as in Eq. 3.11. In the next paragraph, we set $\Delta E=0$ and explore $\Delta \tau$ on the full range $\tau \in[0,1]$. The crossover temperature and the universal behaviors in $T / T_{\text {co }}$ will be discussed afterwards.

\section{Channel asymmetry perturbation $\Delta \tau \neq 0$}

Furusaki and Matveev have proposed a renormalization flow diagram for the conductances in the 'charge' $2 \mathrm{CK}$ model and the $2 \mathrm{CK}$ model with true spin (a particular case of 4CK model) [43]: the channel with the lowest initial bare transmission will end to zero conductance whereas the best initially coupled channel will flow to the (N/2)-CK fixed point (see Fig. 4.6).

Note that the lines of the diagrams are hand sketches between the fixed points (they are not exact calculations). Note also that the 1CK fixed point has not been calculated in [43], and its position at $G_{K}$ is only postulated by the authors of Fig. 4.6. According to our naive reasoning, the 1CK fixed would rather be at $G_{1 \mathrm{CK}}^{*}=0$ (see Fig. 3.8). 

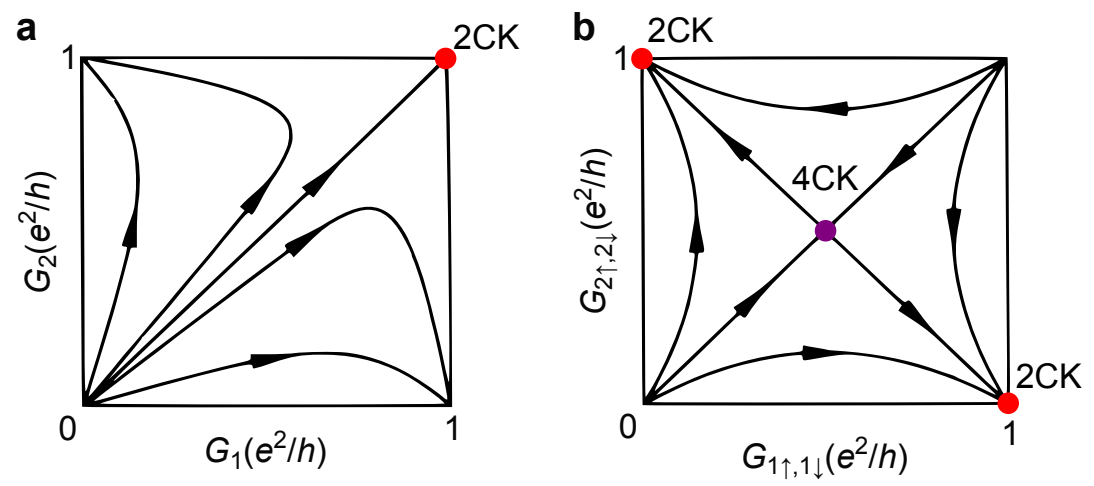

Figure 4.6: Renormalization flow diagram for the 2- and 4-channel 'charge' Kondo effect (reproduced from [43]). The renormalization flow towards the fixed points when lowering the temperature is indicated by the black arrows. The 4CK model (b) corresponds to a $2 \mathrm{CK}$ model (a) with electrons carrying a true spin. The red and purple dots locate the $2 \mathrm{CK}, G_{2 \mathrm{CK}}^{*}=G_{K}$, and the $4 \mathrm{CK}, G_{4 \mathrm{CK}}^{*}=1 / 2 G_{K}$, fixed points respectively. In the $2 \mathrm{CK}$ diagram (a), the position of the $1 \mathrm{CK}$ fixed point $G_{1 \mathrm{CK}}^{*}$ is not certain (see text).

\section{Theoretical prediction for an effective magnetic field perturbation $\Delta E \neq 0$}

Finite $\Delta E$ are observed when measuring Coulomb blockade peaks (conductance versus the gate voltage $V_{g}$ ). The shape and the height of the peak depend on the temperature $T$ and the transmissions $\tau_{i}$ of each channel. In Chapter 3 we focused on the height only (the conductance at charge degeneracy $\delta V_{g}=0$ ); here we will consider the full peak, with symmetric transmissions $\tau \triangleq \tau_{1}=\tau_{2}$.

As explained above, the conductance near the Kondo fixed point $T \ll T_{K}$ is a universal function of $T / T_{\mathrm{co}}$ and its expression $G_{2 \mathrm{CK}}^{T \ll T_{K}}$ is given by Eq. 4.3 . The full expression of $T_{\text {co }}$ (beyond the limit $\Delta E \ll E_{C}$ given in Eq. (4.5)), has been computed by Furusaki and Matveev for $1-\tau \ll 1$ [43]:

$$
k_{B} T_{\mathrm{co}}=\frac{8 \gamma E_{C}}{\pi^{2}}(1-\tau) \sin \left(\pi \Delta N_{g}\right)
$$

where we use $\Delta N_{g} \triangleq \delta V_{g} / \Delta V_{g}=\Delta E / 2 E_{C}$ to simplify. The conductance $G_{2 \mathrm{CK}}^{T \ll T_{K}}$ is plotted in Fig. $4.7 \mathrm{a}$ at $\tau=0.9$ and for different temperatures. Figure $4.7 \mathrm{c}$ includes the predicted $T$-linear deviation from a fully developed quantum criticality (see Eq. (2.9)).

In general, the larger the temperature, the broader the conductance peak. However, in contrast with a standard thermal broadening, here the range of $\Delta N_{g}$ where the conductance is close to $G_{2 \mathrm{CK}}^{*}$ widens with temperature according to a well defined critical exponent. A dashed line is plotted at the crossover conductance $G_{\mathrm{co}} \triangleq 0.5 G_{K}$ used to characterize this effect. This broadening of the quantum crit- 

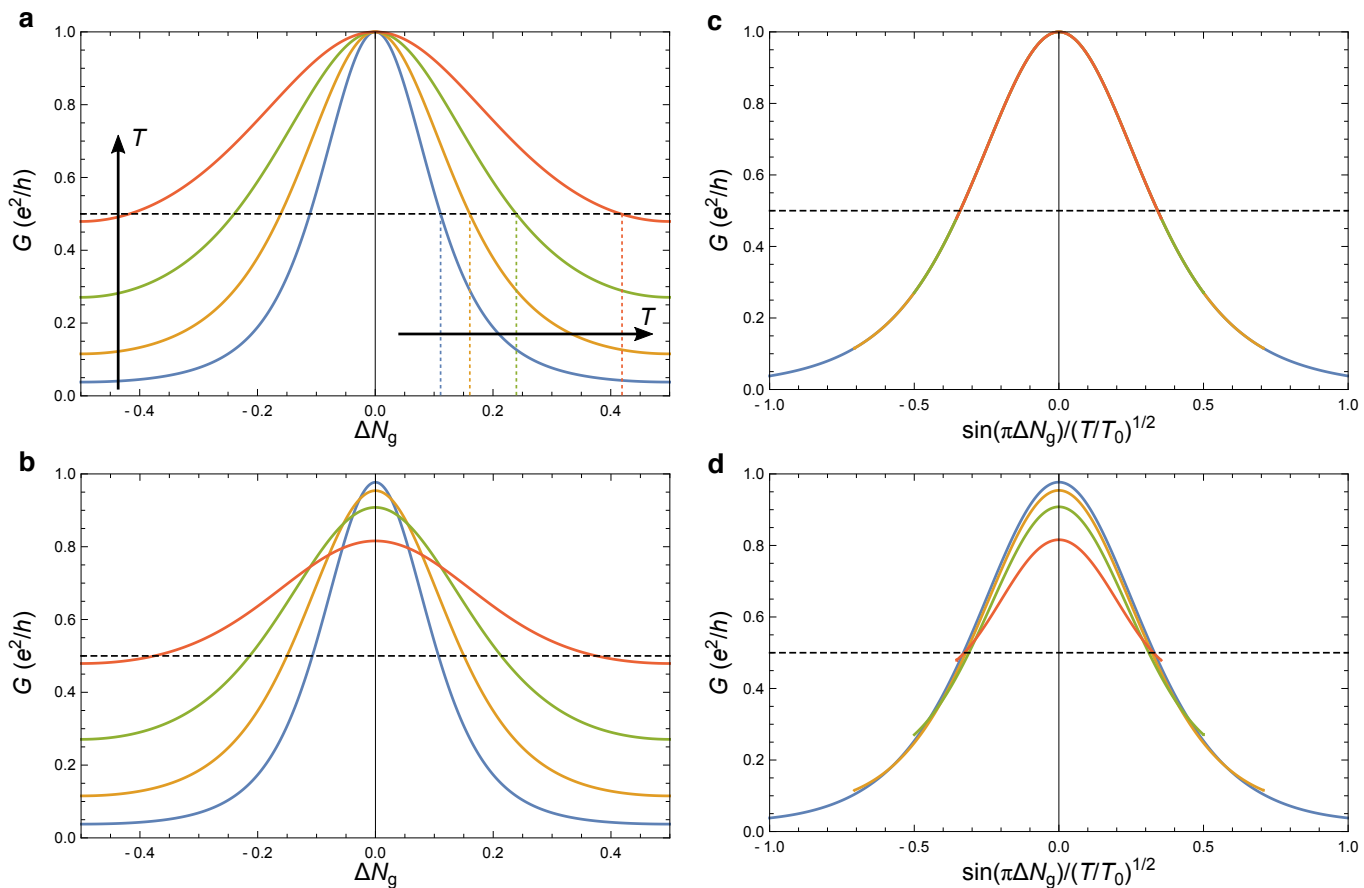

Figure 4.7: Rescaling of a $2 \mathrm{CK}$ Coulomb peak. a, Plot of the conductance $\widetilde{G}_{2 \mathrm{CK}}^{T \ll T_{K}}$ over one period in $\Delta N_{g}$ for $T=\{5,10,20,40\} \mathrm{mK}$ (from bottom to top) with $E_{C} / k_{B}=300 \mathrm{mK}$. The black dashed line indicates $G_{\mathrm{co}}=0.5 G_{K}$. The typical width of the quantum critical area is given by the intersection of the conductance $G$ with $G_{\mathrm{co}}$, it is shown by the vertical colored short dashed lines. b, Same as a, but the $T$-linear term is taken into account. c, The conductance traces shown in a are rescaled (see the $x$-axis). d, Same as c, but the $T$-linear term is taken into account.

ical area with the temperature should agree with the general expression Eq. (4.2) given by Cox and Zawadowski. For $N=2$ channels, the crossover should occur on a temperature scale $T_{\text {co }} \propto \Delta N_{g}^{2}$ for $\Delta N_{g} \ll 1$.

Let us go further and look for a method to check whether the conductance measured experimentally follows a universal function $G\left(T / T_{\mathrm{co}}\right)$. For $\Delta N_{g} \ll 1$, $T_{\text {co }} \propto \Delta N_{g}^{2}$ is a power law; therefore, a method would be to plot $G\left(T / \Delta N_{g}^{2}\right)$, or equivalently $G\left(\Delta N_{g} / T^{1 / 2}\right)$. As we know the general expression of $T_{\text {co }}$, we can use a rescaling that is valid even beyond the limit $\Delta N_{g} \ll 1$ where $T_{\text {co }}$ was a simple power law. We therefore rescale the axis $\Delta N_{g} \mapsto \sin \left(\pi N_{g}\right) / \sqrt{T / T_{0}}$, with $T_{0}$ a temperature of reference. This rescaling has been applied in Fig. 4.7, where one can observe that the curves of Fig. 4.7 a for different temperatures exactly collapse on a single rescaled curve in Fig. $4.7 \mathrm{c}$ on the full range of $\Delta N_{g}$.

The same rescaling is tested in Fig. $4.7 \mathrm{~d}$ on the full expression $G_{2 \mathrm{CK}}^{T \ll T_{K}}$ including the $T$-linear term describing the deviation from a fully developed quantum 
criticality. This term vanishes at $\Delta N_{g}=1 / 2$, hence this rescaling works well on the 'tails' of the conductance peaks. A naive generalization of the scaling to the $N$-channel 'charge' Kondo is:

$$
\Delta N_{g} \mapsto \frac{\sin \left(\pi \Delta N_{g}\right)}{\left(T / T_{0}\right)^{1 / \beta}} \text { with } \beta=\frac{2+N}{N}
$$

where $T_{0}$ is just a temperature scale (in practice we will use the base temperature).

\subsection{Experimental observation of the multi-channel quantum phase transitions}

We will now present our experimental results when we introduce a relevant perturbation in the two- and three-channel 'charge' Kondo effect. This section is divided as follows: (i) first the channel asymmetry $\Delta \tau$ perturbation is discussed, (ii) then a subsection is dedicated to the unexpected phenomena that appear when exploring the channel asymmetry (iii) and finally we consider an effective magnetic field $\delta V_{g}$ perturbation.

\subsubsection{Development of the Kondo QPT versus channel asymme- try $\Delta \tau$}

We have observed a flow towards the predicted fixed points of the two- and threechannel 'charge' Kondo model in Fig. 3.12 of Chapter 3. In that chapter, the transmission had been adjusted to get symmetric channels $\left(\tau_{1}=\tau_{3}\right.$ and $\tau_{2}=0$ to reach the $2 \mathrm{CK}$ fixed point and $\tau_{1}=\tau_{2}=\tau_{3}$ for the $3 \mathrm{CK}$ ). Here we purposely set an asymmetry $\Delta \tau$ between the channel to observe the full renormalization flow of the in-situ conductances of each Kondo channel.

\section{Kondo renormalization flow diagrams}

For the 2CK diagram shown in Fig. 4.8, one needs to open three QPCs to extract the individual conductances of each channel, as explained in Appendix E.1. Indeed, with only two non-zero transmissions $\left(\tau_{1,2}>0\right)$, one has only access to the serial conductance through the two junctions $\left(G_{1} G_{2} /\left(G_{1}+G_{2}\right)\right)$. In this case, we have weakly coupled a third QPC to be able to probe the individual conductances $G_{1}$ and $G_{2}$. In order to minimize the perturbations on the $2 \mathrm{CK}$ physics, we set $\tau_{3} \ll \tau_{1,2}$. Note that the axes do not start at zero because the tunnel configurations $\tau_{3} \ll \min \left(\tau_{1}, \tau_{2}\right) \ll 1$ are hard to access experimentally. In this entire diagram, the 
in situ conductance of the probe channel QPC ${ }_{3}$ obeys $1 / 150<G_{3} / \min \left(G_{1}, G_{2}\right)<$ $1 / 6$. The error bars are based on the statistics from successive Coulomb peaks.

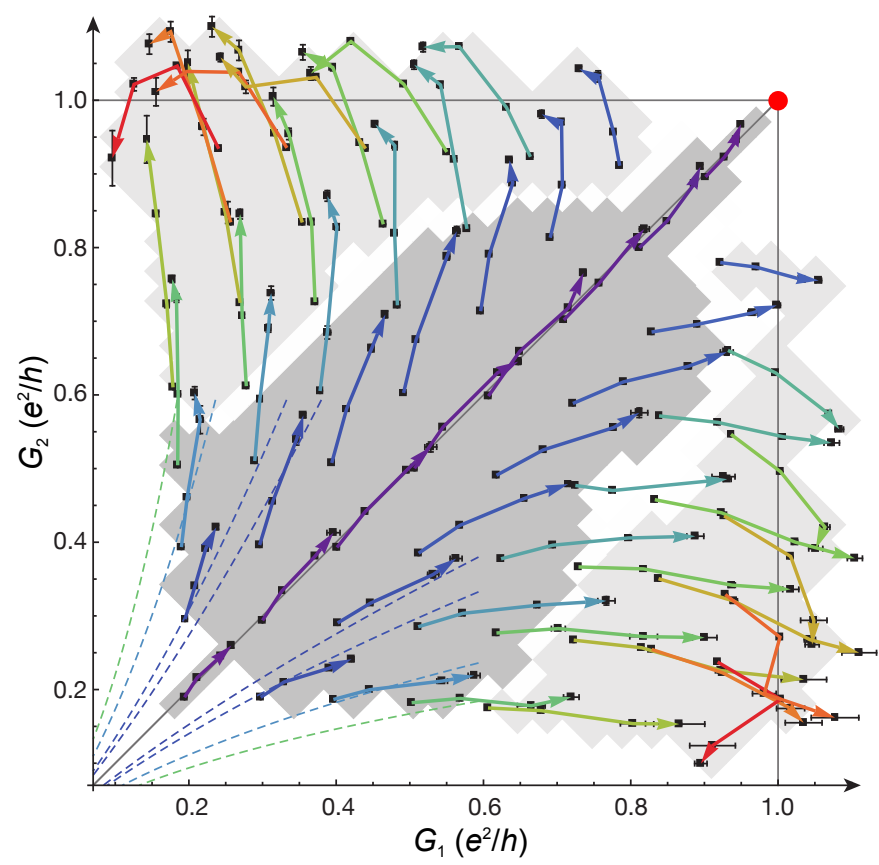

Figure 4.8: Observation of the $2 \mathrm{CK}$ renormalization flow diagram. The insitu conductances $G_{1}$ and $G_{2}$ are measured for temperatures $T \approx\{14,22,38,80\} \mathrm{mK}$ (using $G_{3} \ll G_{1,2}$ as a probe). Each arrow corresponds to a fixed configuration $\left(\tau_{1}, \tau_{2}, \tau_{3}\right)$. The arrows point towards the lowest temperature; their color shows the asymmetry $(\Delta \tau \approx 0$ purple, $\Delta \tau \approx 0.57$ red). Errors bars come from statistics on successive Coulomb peaks. The dashed lines represent a poor man's scaling. The light gray area delimits the region where the weakest transmitted channel decouples (its conductance decrease). The red disk indicates the location of the $2 \mathrm{CK}$ fixed point.

For the $3 \mathrm{CK}$ diagram shown in Fig. 4.9, no probe is required since three channels are involved. In principle, a $3 \mathrm{CK}$ flow diagram should be represented in a $3 \mathrm{D}$ plot. But this will be too long to acquire, very redundant and hardly readable. Hence we decide to set two channels symmetric $\tau_{1}=\tau_{3}$ and plot the data in a $2 \mathrm{D}$ graph $G_{2}$ versus $G_{1,3}$ (where we have averaged ${ }^{5} G_{1}$ and $G_{3}$ ). Most of the data in this graph have an uncertainty on both $G_{2}$ and $G_{1,3}$ smaller than $0.05 G_{K}$, except the data points connected from high temperatures with a dashed

${ }^{5}$ This is possible since they remain approximately equal. The typical difference between $G_{1}$ and $G_{3}$ can be observed on the diagonal of the diagram, from the similar difference between $G_{2}$ and $G_{1,3}$. 
line (whose uncertainty is between $0.05 G_{K}$ and $0.1 G_{K}$, these points are harder to acquire because $G_{1,3}$ is low whereas $G_{2}$ is high).

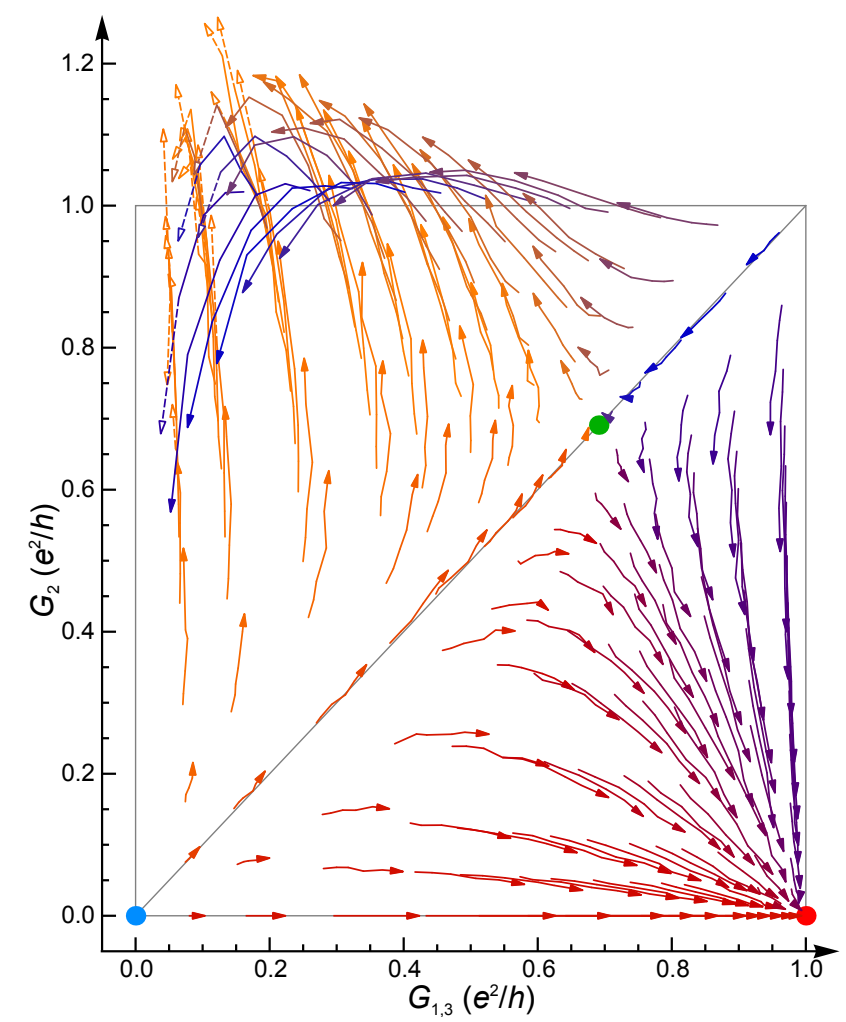

Figure 4.9: Observation of the $3 \mathrm{CK}$ renormalization flow diagram. The averaged conductance of $G_{1}$ and $G_{3}$ is plotted versus $G_{2}$ at temperatures $T \approx\{7.9$, $9.5,12,18,29,40,55\} \mathrm{mK}$. Here, the color of the arrows maps their orientation. The uncertainty on the open symbols and dashed lines is smaller than $0.1 G_{K}$ whereas it is smaller than $0.05 G_{K}$ for the solid symbols and lines. The fixed points are indicated with colored dots (1CK in cyan, $2 \mathrm{CK}$ in red and $3 \mathrm{CK}$ in green).

\section{Tunnel case $\tau \ll 1$}

In the multi-channel Kondo model, channels weakly coupled to the impurity (when $T \gg T_{K}$ ) are renormalized independently. This is shown in the 2CK diagram where the dashed lines correspond to the poor man's scaling defined ${ }^{6}$ by Eq. (3.8). Each line is associated to the arrow it is touching (and which is of the same color). One

${ }^{6}$ To be precise, we plot $G_{2}^{T \gg T_{K}}\left(T / T_{K}\left(\tau_{2}\right)\right)$ versus $G_{1}^{T \gg T_{K}}\left(T / T_{K}\left(\tau_{1}\right)\right)$, where $G^{T \gg T_{K}}\left(T / T_{K}\right)=$ $19.24 G_{K} \ln ^{-2}\left(T / 0.0037 T_{K}\right)$ is the tunnel regime of the universal curve (see Fig. 3.14 a), and where $T_{K}\left(\tau_{1}\right)$ and $T_{K}\left(\tau_{2}\right)$ correspond to the $T_{K}(\tau)$ in the inset of the same figure. 
can see that this approximate scaling works quite well although the transmission are not really tunnel-like.

\section{Symmetric coupling $\Delta \tau=0$}

This case was the topic of Chapter 3 where the flow towards the two- and threechannel Kondo fixed point was studied quantitatively. Here we directly show that symmetric channels remain symmetric under the renormalization flow. Note that the symmetry was fine tuned to symmetric couplings at low temperature $T \approx 18 \mathrm{mK}$. The procedure is explained in Appendix E.2.

\section{Development of a quantum phase transition when $\Delta \tau \neq 0$}

At zero temperature, the conductances should reach one of the three fixed points depending on the initial setting on the transmissions. Figures 4.8 and 4.9 show the development of a quantum phase transition near the $\Delta \tau=0$ line as the temperature is reduced. In the $2 \mathrm{CK}$ diagram, the dark gray area contains the points which show an increase of both $G_{1}$ and $G_{2}$, whereas in the light gray area one of the conductances is decreasing.

In the 3CK diagram, the influence of the different fixed point (marked with colored dots) is even more obvious. Moreover it displays two quantum critical points (the $2 \mathrm{CK}$ and the $3 \mathrm{CK}$ fixed points). One can then observe a remarkable crossover from a NFL fixed point (the $3 \mathrm{CK}$ ) to another NFL fixed point (the $2 \mathrm{CK}$ ).

\subsubsection{Unanticipated features of the flow diagrams}

The renormalization flow diagrams shown in Figs. 4.8 and 4.9 display some previously unanticipated features: Whereas the flow to the two- and three-channel Kondo fixed points appears regular, the flow towards the one-channel Kondo state shows an overshoot of the in situ conductance above the free electron quantum limit and an unanticipated conductance at the $1 \mathrm{CK}$ fixed point $G_{1 C K}^{*}=0$. We right away point out that these striking features are now corroborated by novel NRG calculations.

\section{The 'charge' $1 \mathrm{CK}$ fixed point $G_{1 \mathrm{CK}}^{*}=0$}

In the previous chapter, we discussed a naive reasoning to get $G_{1 \mathrm{CK}}^{*}=0$ from a non-monotonous relation between $J$ and $\tau$ (see Fig. 3.8 on page 57). This position of the 1CK fixed point contradicts the diagram sketched by Furusaki and Matveev (see Fig. 4.6). 
The $1 \mathrm{CK}$ limit is not visible in the $2 \mathrm{CK}$ diagram because we need $G_{3} \ll$ $\min \left(G_{1}, G_{2}\right)$ (in order to consider $\mathrm{QPC}_{3}$ as non-invading probe). However, the signal-to-noise ratio is good enough in the 3CK diagram in the limit $\tau_{1,3} \ll \tau_{2}$. We observe that the arrows in this limit (the ones starting from the top of the diagram) are pointing towards zero-conductance at low temperature. Moreover, some arrows in the region $G_{1,3} \approx 0.2$ show a non-monotonous behavior of $G_{2}$ (at large $G_{2}$ ).

These two observations are in agreement with the naive picture we propose. They are now also supported by NRG calculations 7 .

\section{Renormalization flow diagram with $\tau_{2}=1$}

The limit $\tau_{2}=1$ can be reached by setting the $\mathrm{QPC}_{2}$ in the middle of the first IQHE plateau. The transmission in this case is perfectly equal to one within our measurement accuracy (see the discussion on the reflectivity at the 2DEG-metallic island interface in Appendix B.2). In this case, the transmission of the channel is said ballistic, and one naively expects a conductance $G_{2}$ equal to the quantum of conductance $G_{K}$ whatever the environment of $\mathrm{QPC}_{2}$ (i.e. whatever $\tau_{1}, \tau_{3}$ and $E_{C}$ ) and whatever the temperature 8 (below a reasonable limit).

In Fig. 4.10 we present the flow diagram for $\tau_{2}=1$ (with $\tau_{1}=\tau_{3}$ ). As we have demonstrated in Chapter 2, there is no more charge quantization at $\tau_{2}=1$. Hence the charge degeneracy $\delta V_{g}=0$ has no more meaning. What is plotted in this diagram is the conductance averaged while sweeping $V_{g}$ (to increase the signal-tonoise ratio). We observe a clear drop of the conductance $G_{2}$ when lowering the temperature!

In the same Figure 4.10, the circle and square symbols correspond to the 'reciprocal' situation where $\tau_{1,3}=1$ (with $0<\tau_{2}<1$ ) at the base temperature only. In this case we observe that both $G_{1}$ and $G_{3}$ remain close to $G_{K}$ (at our experimental accuracy).

The drop in the in-situ conductance $G_{2}$ is quite surprising since one would naively expect that a quantum conductor at unitary transmission $\tau=1$ behaves as a classical resistor with a resistance $R=R_{K}$. In particular, the quantum shot noise is known to vanish at this value of the transmission as for a classical resistance. We see that this picture matches the data when two channels are set to $\tau=1$ but not with a single channel.

We have tried to substitute the role of $\mathrm{QPC}_{2}$ by $\mathrm{QPC}_{1}$ or $\mathrm{QPC}_{3}$ (see the 'cross', 'plus' and 'star' symbols in Fig. 4.10). We made the measurement only at base

\footnotetext{
${ }^{7}$ A.K. Mitchell, private communication.

${ }^{8}$ For example, see the Figure 3 of [99], where the conductance of a QPC in series with an ohmic resistor of resistance $R \approx 26 \mathrm{k} \Omega \approx R_{K}$ does not drop at low temperature in the limit of ballistic transmission $\tau_{\infty} \longrightarrow 1$
} 


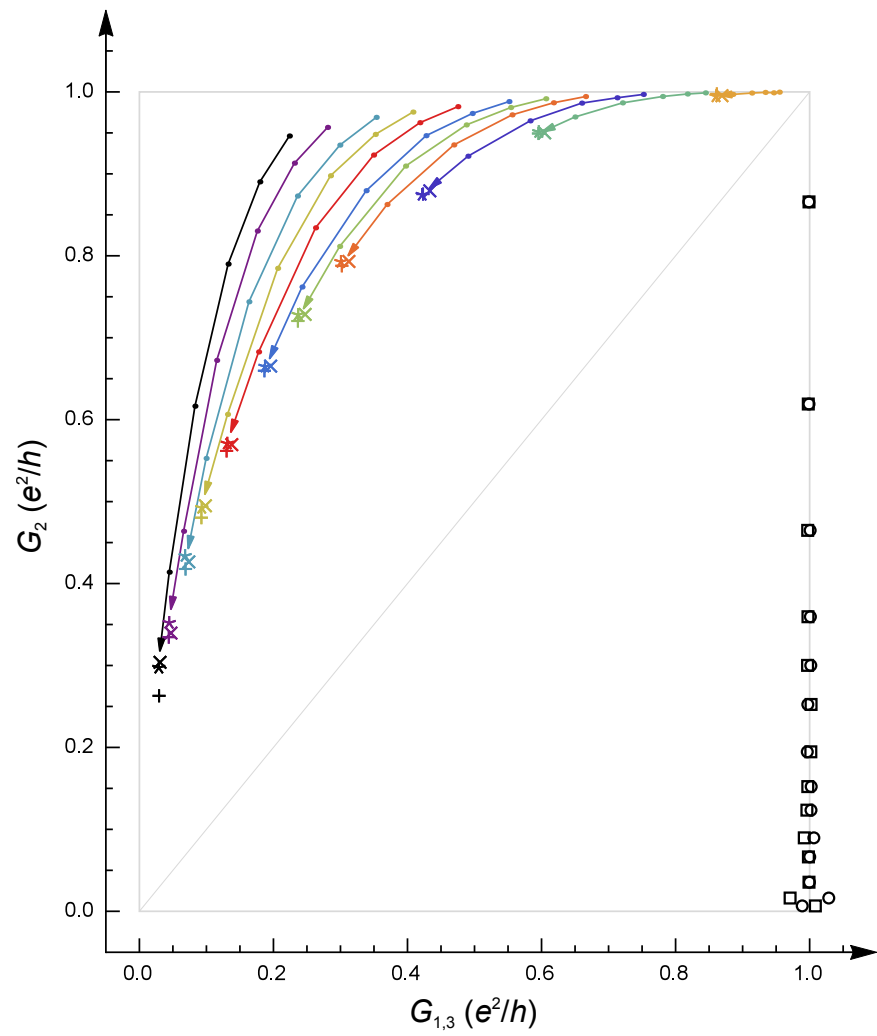

Figure 4.10: Renormalization flow of the in-situ conductances when some transmissions are set at $\tau=1$. Each point is an average over $\delta V_{g}$ (see text). The arrows show the in-situ conductances $G_{2}$ versus $G_{1,3}$ at $\tau_{2}=1$ for $T \approx\{8.9,9.5$, $12,18,29,40\} \mathrm{mK}$. For comparison, the role of $\mathrm{QPC}_{2}$ has been replaced by $\mathrm{QPC}_{1}$ (cross), $\mathrm{QPC}_{2}$ (plus) and $\mathrm{QPC}_{3}$ (star) at $T \approx 8.0 \mathrm{mK}$. The squares and the circles are measured at $T \approx 8.0 \mathrm{mK}$ only, at $\tau_{1}=\tau_{3}=1$ for different values of $\tau_{2}$. The squares correspond to $G_{1}$ and the circles to $G_{3}$.

temperature and we have observed that the conductance of the channel which is set to $\tau=1$ does not depend on the QPC. This observation shows that the conductance drop cannot be explained with a residual reflection due to a $\tau$ strictly lower that one. Indeed, the residual reflection would not be exactly the same for the three QPCs.

\section{Arrow crossings and universality}

In the $3 \mathrm{CK}$ renormalization flow diagram, we observe many arrow crossings (in the upper part $\left.\tau_{2}>\tau_{1,3}\right)$. These crossings also exist in the 2CK diagram, but are less spectacular, barely above noise. They are in an apparent contradiction with the universal behavior expected at low temperature $k_{B} T \ll E_{C}$. Indeed, if 
$\left(G_{2} ; G_{1,3}\right)$ fully parametrize the universal flow, the trajectories cannot cross.

However, these crossings do not necessarily imply a non-universal behavior if $G$ do not completely characterize the renormalization flow. We expect it to be the case as the relation between $J$ and $G$ is non-monotonous in absence of interaction (see Fig. 3.8 on 57). Therefore, a universal flow diagram with the parameter $J$ would contain crossings when plotted versus $G$.

In the lower part $\left(\tau_{2}<\tau_{1,3}\right)$ of the diagram, the arrows do not cross each other, at the measurement accuracy (in agreement with the monotonous relation between $G$ and $J$ expected along the flow to the $2 \mathrm{CK}$ fixed point, which is located at the maximum of the function $G(J))$.

\section{Overstepping the quantum of conductance}

The observation of an in situ conductance of a single channel larger than the quantum of conductance $e^{2} / h$ is a striking property of both the 2- and 3-CK flow diagrams. It is especially clear in the $3 \mathrm{CK}$ measurement where the signal-to-noise ratio is better. This observation relies on fine calibrations of the offset and the gain of the conductance measurement (see Appendix E.1.2). This allowed us to plot data with accuracy better than $0.05 G_{K}$ (plain arrows) and avoid any doubt about the actual overstepping of $G_{K}$.

One should also notice that the overstepping of $G_{2}$ above $G_{K}$ increases as $\tau_{2}$ is lowered. Note that there is no overstep in the opposite limit $\tau_{2}=1$ (see Fig. 4.10).

From a general point of view, it is certain that no more than a single quantum Hall edge channel is involved at each QPC since the voltages applied on these split gates completely reflect the other channels. The way we extract the in situ conductances from the classical conductance composition laws is explained in Appendix E.1. Note that the measured conductance (per channel) through the whole device does not overstep the quantum limit $G_{K}$ because of the low conductance of $\mathrm{QPC}_{1}$ and $\mathrm{QPC}_{3}$.

Some preliminary NRG calculations of A.K. Mitchell corroborate these observations. It means that this effect is included in the Kondo model. However, to date, we have no simple picture to explain this property.

\subsubsection{Crossover from criticality versus detuning from charge degeneracy $\delta V_{g}$}

We now set $\Delta \tau=0$ and explore the perturbation due to an effective magnetic field $\delta V_{g}$. In practice, this consists in measuring the conductance per channel $G$ versus the plunger gate $V_{g}$ over one period $\Delta V_{g} \approx 0.70 \mathrm{mV}$. 


\section{Quantum criticality in the Kondo renormalized conductance peaks}

Let us start with checking that the parameter space of quantum criticality grows with the temperature, according to the predicted power law. A conductance peak in the 2CK regime at $\tau \approx 0.90$ is plotted for different temperatures in Fig. 4.11 . This figure has to be compared to Fig. $4.7 \mathrm{a}$ and $\mathrm{b}$.

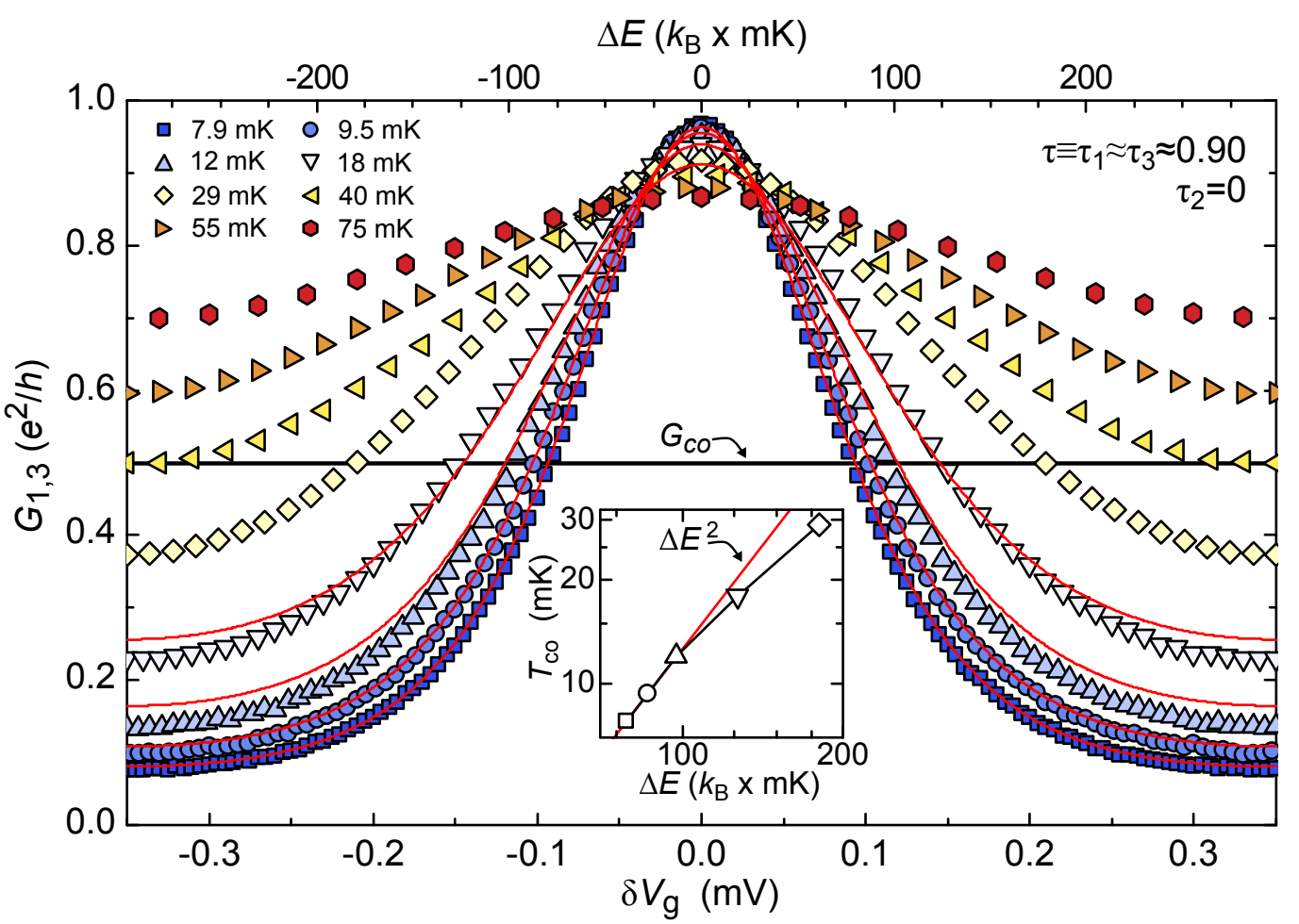

Figure 4.11: Quantum criticality in a $2 \mathrm{CK}$ conductance peak. Coulomb peaks are displayed on a full period $\Delta V_{g}=0.70 \mathrm{mV}$ for different temperatures (each symbol corresponds here to a temperature, see the legend) in the $2 \mathrm{CK}$ regime. The red lines are the prediction Eq. (4.3) plotted for the independently measured parameters. The horizontal black line is placed at $G_{\mathrm{co}} \triangleq 0.5 G_{K}$. The intersection of $G_{1,3}$ with this line gives the $T_{\mathrm{co}}(\Delta E)$ shown in the inset as symbols. The red straight line corresponds to the $2 \mathrm{CK}$ power law $T_{\mathrm{co}} \propto \Delta E^{2}$, it is adjusted to match the lowest temperature (square) symbol.

Fig. 4.11 contains a double $x$-axis: one can read both (i) the $\delta V_{g}$ applied in practice (bottom) and (ii) the corresponding splitting in energy level when breaking the charge degeneracy $\Delta E \triangleq 2 E_{C} \delta V_{g} / \Delta V_{g}$ (top).

This peak broadening reflects the widening range of $\Delta E$ for quantum criticality as the temperature is increased. It can be characterized by the intersection of the conductance with $G_{\mathrm{co}}$ (black horizontal line): this crossing occurs by 'definition' at 
$T=T_{\mathrm{co}}(\Delta E)$. In the inset of Fig. 4.11, we have plotted this crossover temperature $T_{\text {co }}$ (up to $29 \mathrm{mK}$ ) versus $\Delta E$ and compared it to the power law $T_{\text {co }} \propto \Delta E^{2}$ expected for the $2 \mathrm{CK}$ effect.

\section{Observation of a power law on the crossover temperature $T_{\mathrm{co}}$}

In Fig. 4.12 we have generalized the procedure shown in Fig. 4.11 to other transmissions and also to the $3 \mathrm{CK}$ measurement. We observe deviations from the predicted power laws at high temperatures. We know from Chapter 3 that nonuniversal behaviors occur at temperatures higher than $T \approx 12 \mathrm{mK}$ (see Fig. 3.14). Therefore, we have distinguished the three lowest temperatures in this figure (solid versus open symbols). The inset shows an extraction of the critical exponent $\beta$ based on a fit of the data at $T \leq 12 \mathrm{mK}$ to a power law $\Delta E^{\beta}$. When we average over all the transmissions, we find $\beta=1.94 \pm 0.04$ in the $2 \mathrm{CK}$ regime (the red line is at the value $\beta=2$ predicted for $2 \mathrm{CK}$ ) and $\beta=1.70 \pm 0.07$ in the $3 \mathrm{CK}$ regime (the green line is at $\beta=5 / 3 \approx 1.667$, as predicted for $3 \mathrm{CK}$ ). Although these two power laws are relatively close, we are clearly able to discriminate between the $2 \mathrm{CK}$ and $3 \mathrm{CK}$ predictions.

\section{Universality of the conductance expressed in $T / T_{\mathrm{co}}$}

We can go further and verify that once the temperature has been lowered enough to reach the non-Fermi liquid regime $\left(T \ll T_{K}\right)$, the only energy scale to consider is the crossover temperature $T_{\text {co }}$ (provided $k_{B} T \ll E_{C}$ ).

As it has been explained in Section 4.2.2, we have a method to check whether the conductance follows a universal function of $T / T_{\text {co }}$ on a full range of $\Delta E$. The $a d$ hoc function to use is $\Delta E \mapsto \sin \left(\pi \Delta E /\left(2 E_{C}\right)\right) /\left(T / T_{\text {base }}\right)^{1 / \beta}$ where $T_{\text {base }}=7.9 \mathrm{mK}$ is the base temperature of the experiment (see Eq. (4.7)). Using this rescaling, we observe in Fig. 4.13 that the conductance peaks at all temperatures collapse on a single curve (which is given by Eq. (4.3) for the 2CK, see Fig. 4.7).

The conductance peak displayed for the $3 \mathrm{CK}$ rescaling is the closest to the $3 \mathrm{CK}$ fixed point ( $\tau \approx 0.83$ ). Therefore, its height does not depend on the temperature (except for the red and the orange traces that deviate from the universal regime defined by $T \ll E_{C}$ ).

With the scalings showed in Fig. 4.13, we verified that in the vicinity of the two- and three-channel Kondo quantum critical points, the conductance follows a universal function of $T / T_{\mathrm{co}}$ on the full range of $\Delta E$. 


\subsection{Conclusion}

In this chapter, we have studied the finite temperature signatures of the secondorder quantum phase transition that occurs at the overscreened multi-channel Kondo fixed points. We have used in turn a channel asymmetry and an effective magnetic field as a relevant perturbation.

On the one hand, breaking the channel symmetry allowed us to observe the full renormalization flow diagrams of the conductance. These diagrams illustrate the development of a quantum phase transition as the temperature is lowered. The convergence towards the $1 \mathrm{CK}$ fixed point displays a remarkable overstepping of the quantum of conductance $e^{2} / h$ by the in situ conductance of a single electronic channel (now corroborated by preliminary NRG calculations). We have also observed that the in situ conductance of an electronic channel accurately set at transmission $\tau=1$ can be reduced towards zero under the renormalization process.

On the other hand, considering the voltage gate $V_{g}$ used to measure conductance peaks as an effective magnetic field, a crossover from quantum criticality ( $\delta V_{g}=0$ at $T \ll T_{K}$ ) can be studied. The broadening of these conductance peaks is in agreement with a temperature scale that follows a universal prediction $T_{\mathrm{co}} \propto$ $\delta V_{g}^{(2+N) / N}$ valid whatever the nature of the degrees of freedom used to implement the $N$-channel Kondo model. More generally, beyond the limit of small $\delta V_{g}$, we have observed that both $2 \mathrm{CK}$ and $3 \mathrm{CK}$ Coulomb peaks for different temperatures $T$ collapse onto a single curve when plotted versus a rescaled expression of $\delta V_{g} \mapsto$ $\sin \left(\pi \delta V_{g} / \Delta V_{g}\right) /\left(T / T_{\text {base }}\right)^{1 / \beta(N)}$. The ad hoc sine term used in this expression generalizes Furusaki and Matveev's derivation of the zero temperature conductance in the strong coupling limit of the 'charge' $2 \mathrm{CK}$ model. 

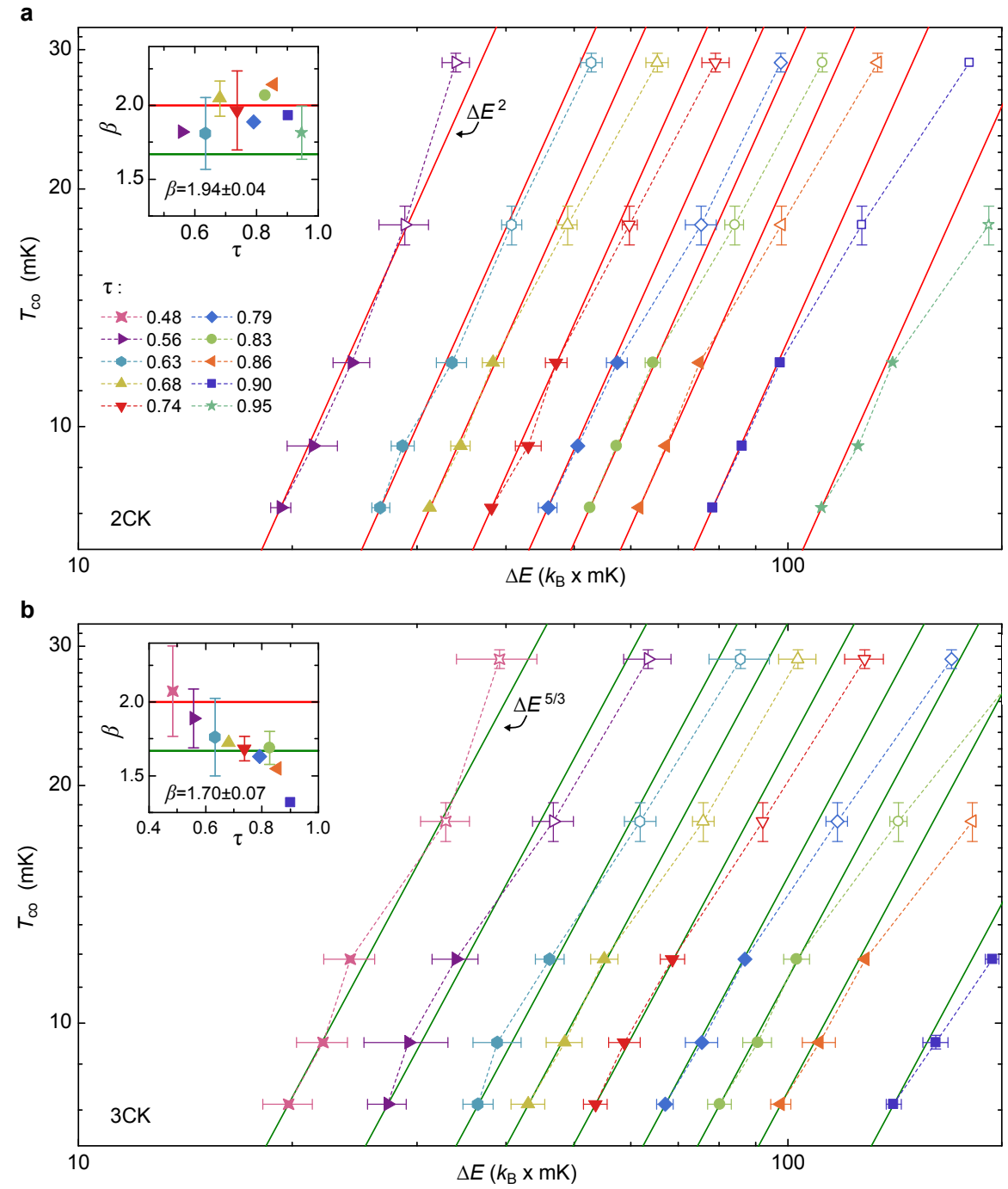

Figure 4.12: Power law on the crossover temperature $T_{\text {co }}$ versus $\Delta E$. $T_{\text {co }}$ is plotted versus $\Delta E$ for different transmissions (see legend in a) in the $2 \mathrm{CK}$ (a) and $3 \mathrm{CK}$ (b) regime. The predicted power law (red for $2 \mathrm{CK}$, green for $3 \mathrm{CK}$ ) is adjusted to match the lowest temperature $T_{\text {base }}=7.9 \mathrm{mK}$. In the inset of each graph, we show a fit of the critical exponent $\beta$ for each transmission $\tau$ for the three lowest temperatures (shown as solid symbols in the main graphs). The predicted exponents ( $\beta=2$ in red for the $2 \mathrm{CK}$ and $\beta=5 / 3$ in green for the $3 \mathrm{CK}$ ) are plotted for comparison with the mean value obtained over all the transmissions $\tau$. 

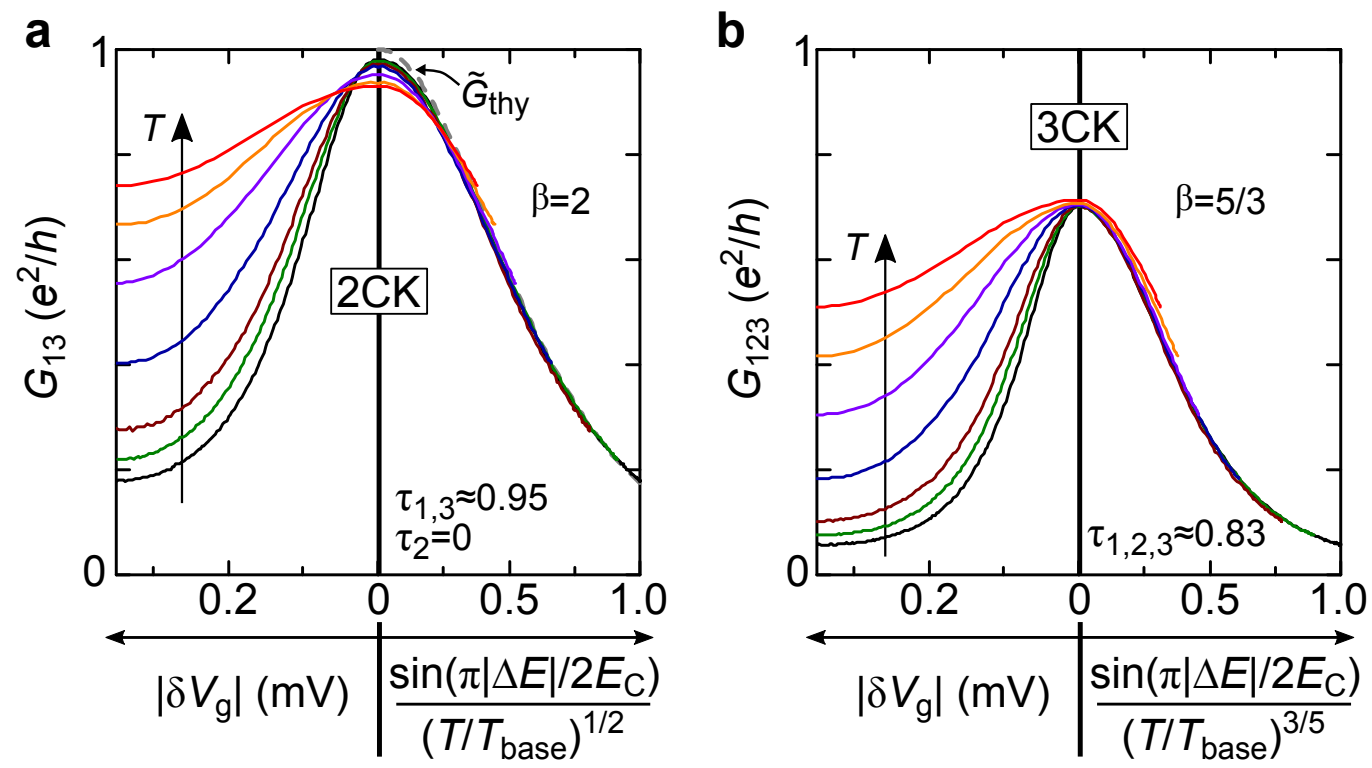

Figure 4.13: Rescaling of a conductance peak. A conductance peak is shown at $T \approx\{7.9,9.5,12,18,29,40,55\} \mathrm{mK}$ for a selected transmission $\tau$ close to the $2 \mathrm{CK}$ (in a) and the $3 \mathrm{CK}$ (in $\mathbf{b}$ ) fixed points. The solid lines are experimental data. For each graph $\mathbf{a}$ and $\mathbf{b}$, two scales are used: the raw plunger gate voltage $\delta V_{g}$ (left) and a rescaled axis $\sin \left(\pi \Delta E /\left(2 E_{C}\right)\right) /\left(T / T_{\text {base }}\right)^{1 / \beta}$ (right). The conductance adopts a universal behavior in the rescaled axis (except for the highest two temperatures). The gray dashed line in a shows the zero temperature prediction for $\tau=0.95$. 


\section{Chapter 5}

\section{Outlook}

This concluding chapter is written as a list of possible further research directions.

\section{Charge quantization and multi-channel Kondo effect in the frac- tional quantum Hall effect regime}

As shown in Fig. A.4, the 2DEG we use displays a quantum Hall plateau at the fractional filling factor $v=2 / 3$. The behaviors of the QPCs and the connection of the electronic channels to the central micron-size ohmic contact are probably different at this filling factor. However, it would be very interesting to measure the destruction of the charge quantization and the renormalization flow of the conductance towards the multi-channel Kondo fixed point in the FQHE regime.

\section{Noise measurement near the quantum critical Kondo fixed points}

Measuring the noise on the current can give access to the statistics of the charge carriers [108]. The non-Fermi liquid physics near the quantum critical points of the overscreened Kondo fixed point could lead to a fractionalization of the charge. Probing this exotic effect should be possible effect with shot noise measurements. This noise vanishes in principle near the $2 \mathrm{CK}$ fixed point (which is located at ballistic transmission). However the $3 \mathrm{CK}$ fixed point should be accessible.

\section{Two-impurity sample}

The sample studied in this thesis counts a single pseudo-spin Kondo impurity. Nanofabricating a new sample with two metallic islands separated by a QPC may implement the two-impurity and the multi-channel two-impurity 'charge' Kondo model. The two-impurity model is important as it includes both an RKKY interaction between the impurity spins and the usual Kondo interaction between 
each impurity spin and the conduction electrons [54]. This is a simplified version of the Kondo lattice which is central for heavy fermions [44]. 


\section{Appendix A}

\section{Pre-requisites on the quantum transport of electricity}

This chapter will introduce the reader to some basic ideas on the quantum transport of electricity. At first, it would appear as an impossible task to describe the fundamental properties of the transport of electrons in condensed matter. Indeed, since electrons are charged particles, they can interact with the medium and with the other electrons. Moreover, if we consider the 'quantum' problem then it is hopeless to look for a solution by solving the Schrödinger equation of this many body problem [69].

Despite the apparent complexity of the problem, it appears that the solution is sometimes very simple. For instance, the conductance of a $2 \mathrm{D}$ electronic system measured at low temperature and under a strong magnetic field is $v e^{2} / h$ where $v$ is an integer called filling factor, $e$ is the elementary charge and $h$ is the Planck's constant. This phenomenon called 'integer quantum Hall effect' was discovered in 1980 [127]. The fact that this conductance only depends on an integer $v$ and some fundamental constants is absolutely remarkable! This is the kind of experiments discussed in this thesis: the experimental observation of simple $\rrbracket^{1}$ results emerging from a problem which is a priori non-trivial.

This appendix is organized in two sections the first one aims to understand the notions of electronic channel and its maximal conductance (for free electrons): the quantum of conductance $G_{K} \triangleq e^{2} / h$. The second part is dedicated to the study of some important quantum transport phenomena (quantum Hall effect, dynamical Coulomb blockade and the quantum shot noise).

${ }^{1}$ A more physical word would be 'universal', meaning independent of the microscopic details of the sample we consider. 


\section{A.1 Quantum conductors}

Quantum mechanics gives to the electrons a wave-particle duality. In clean samples and at low temperatures, inelastic scattering with impurities and vibrational phonons are rare, the electron phase is thus conserved over long distances. Transport properties will change due to this phase coherence giving rise to observable quantum phenomena.

In this section we focus on quantum conductors and QPC in particular. Indeed, this is the basic building block of our circuits. We are able to put such a conductor inside a circuit just as a usual electrical dipole. However, its behavior is quite singular. First, its conductance (the invert of the electrical resistance) shows steps quantized in units of the quantum of conductance $G_{K} \triangleq e^{2} / h$, when plotted versus a voltage gate that control the size of the QPC. Second, its conductance between two steps depends on the other elements of the circuit (e.g. classical resistors or other quantum conductors).

The first part introduces the quantum of conductance and the notion of electronic channels. The second one presents our experimental realization of quantum conductors.

\section{A.1.1 Quantum of conductance}

A practical way to describe the transport of electrons in a sample with well localized scatterers has been first proposed by Landauer [67]. It has been refined [23, 21], in particular after experimental observations [128, 122, 129] to eventually give the Landauer-Büttiker formalism.

In the first paragraph, we consider an ideal one-dimensional conductor connecting two electronic reservoirs at thermodynamic equilibrium. In the second paragraph, we consider a more practical situation and discuss the Landauer-Büttiker formalism.

\section{Dimensionality}

Let us consider two reservoirs (the left and the right) connected by a pure ideal 1D conductor. The electrical current through the conductor is given by the elementary charge $e$ of an electron times the number of electrons that crosses a section of this conductor per unit of time: $I=e \times \int \frac{\mathrm{d} k}{2 \pi} v(k) f(k)$, where $k$ is the (scalar) wave vector associated to an electron, $v(k)$ is the velocity of an electron carrying a momentum $k$ and $f(k)$ counts the number electrons that carry a momentum $k$ [91]. Assuming free propagation, the energy $E$ reduces to the kinetic energy, thus $\partial E / \partial k=\hbar v$; then the velocity simplifies and it comes: 


$$
I_{L}=\frac{e}{h} \int \mathrm{d} E f_{L}(E)
$$

where $I_{L}$ is the current injected by the left reservoir. For an electronic reservoir at equilibrium (at a temperature $T$ and a chemical potential $\left.\mu_{L}\right), f_{L}(E) \triangleq\{\exp ((E-$ $\left.\left.\left.\mu_{L}\right) / k_{B} T\right)+1\right\}^{-1}$ is given by the Fermi-Dirac distribution. A net current $I$ will flow when one sets a voltage bias $V$ between the left and the right reservoirs $\left(e V=\mu_{L}-\mu_{R}\right)$. Whatever the temperature, the conductance $G=I / V$ in the limit $V \longrightarrow 0$ takes the value of the quantum of conductance:

$$
G_{K}=\frac{e^{2}}{h}
$$

This is the electric conductance of an ideal single-mode conductor. It is limited to the universal value $G_{K} \approx 1 / 25.8 \mathrm{k} \Omega$ by the fermionic nature of the charge carriers. In practice, one may consider non-ideal conductors (embedding scatterers or with a finite width that allow several electronic modes to pass). These situations are discussed in the next paragraph.

\section{Landauer-Büttiker formalism}

When quantum conductor contains defects or irregularities, an incoming electron wavefunction can be either transmitted through the conductor or reflected back to the emitting reservoir. This scattering process is elastic since it is associated to a time-independent potential.

We consider a quantum conductor with a finite width that allows several transverse modes (also called electronic channels). One can build the scattering ${ }^{2}$ matrix $S$ that describes the scattering of the incoming waves (from the left and from the right) into the outgoing waves (to the left and to the right reservoirs) [23]. The conductance is then given by the two-terminal Landauer-Büttiker formula at zero temperature:

$$
G=\frac{e^{2}}{h} \sum_{n} \tau_{n}
$$

where the energy dependence of the transmission probabilities $\tau_{n}$ of the electronic channels is assumed to be negligible. Each channel contribute at most to one quantum of conductance $e^{2} / h$.

Many channels can be transmitted simultaneously and give rise to a larger conductance. However, as it will be explained later, in our case, the opening of the channels occurs one-by-one (for an illustration, see Fig. A.3. In the experiments

${ }^{2}$ In our experiments, we consider a scattering by a smooth potential (a saddle-point constriction). 
reported in this thesis, we have generally $\tau_{1} \leqslant 1$, therefore the $\tau_{n>1} \lll 1$ are negligible.

According to Eq. (A.3), the average current across a quantum conductors is fully characterized by the transmissions $\tau_{n}$ of its electronic channels. Let us see how to build a quantum conductor in practice, and how to control the transmission probabilities $\tau_{n}$.

\section{A.1.2 Quantum coherent conductors}

Quantum coherent conductors can be fabricated using various methods. Our way is to take a $2 \mathrm{D}$ conductor and then to make a constriction of this $2 \mathrm{D}$ conductor into a local 1D conductor. That kind of small $1 \mathrm{D}$ conductor made in a semiconductor is called a quantum point contact (QPC), we will describe its principle in this subsection.

\section{D electron gas (2DEG)}

The most direct example of 2D electron gas (2DEG) is probably a mono-layer of carbon (graphene). However, the graphene technology is not yet mature to implement QPCs (despite promising solutions [137]).

The 2DEG we have used is made in GaAs using MBE techniques at C2N/LPN by Ulf Gennser, Antonella Cavanna and Abdelkarim Ouerghi. This technique consists in evaporating gallium, arsenic and some amount of aluminum on an initial substrate of GaAs. The crystalline growth is controlled with an accuracy which is better than a single layer deposition. This process is done in an ultra high vacuum chamber to reduce pollution.

One can engineer the conduction band of a stack of GaAs/AlGaAs called hetero-structure by modulating the aluminum proportion [11, 114]. The silicon impurities introduced in the AlGaAs region (see Fig. A.1) ionize. The generated free electrons find the minimum of energy $E\left(z_{\min }\right)$ and eventually stay there and form the 2DEG.

Since they are trapped in $z$ direction, their motion will be quantized along this axis. When the temperature is lower than the energy spacing between the modes, only the fundamental level is populated (in black in Fig. A.1 a). They are free to move in the other directions $(x, y)$, but their position in $z$ is locked 3

\footnotetext{
${ }^{3}$ The ground state wavefunction shown in Fig. A.1 gives the average position, $\bar{z}$, of the electron in the quantum well. This position can fluctuate, according to Quantum Mechanics, but the motion remains fundamentally two-dimensional, as the average position cannot change.
} 
a

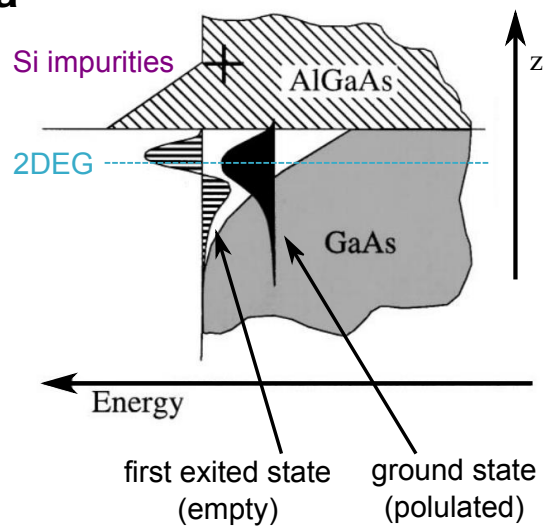

b

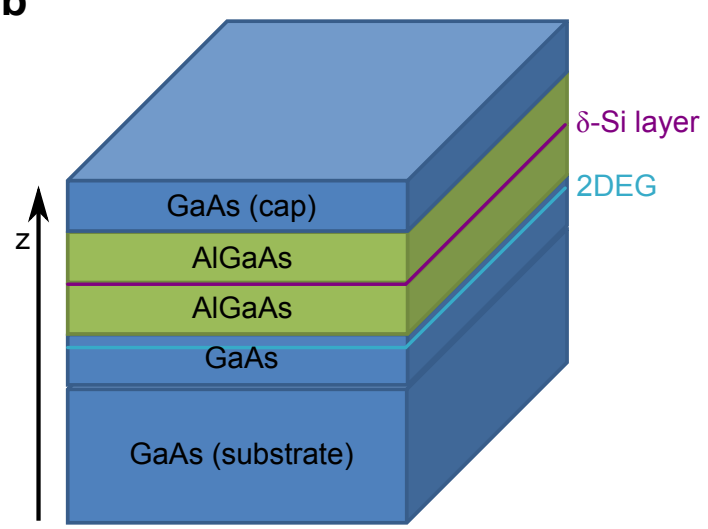

Figure A.1: 2DEG formed in a GaAs/AlGaAs heterostructure. The $z$ axis shows the direction of the MBE growth. a, (reproduced from [114]) The electrons given by the silicon impurities (+) fall in the triangular well. The ground and first excited states wavefunctions are represented. At low temperature, only the quantum ground state is populated. The position of these electrons is locked in an $(x, y)$ plane close to the interface GaAs/AlGaAs. b, Schematic view of our heterostructure. A GaAs cap is used to avoid oxidation. The 2DEG is typically $100 \mathrm{~nm}$ below the surface.

\section{Quantum point contact (QPC)}

What we actually need is a $1 \mathrm{D}$ conductor. The idea is then to polarize split gates deposited on the top of the $(\mathrm{Al}) \mathrm{GaAs}$ heterostructure to build a constriction of the 2DEG and eventually get a 1D conductor. This 1D component is called a QPC. The typical gap between the split gates is given by the Fermi wavelength $\lambda_{F}=\sqrt{2 \pi / n} \approx 50 \mathrm{~nm}$, where $n \approx 2.5 \times 10^{11} \mathrm{~cm}^{-2}$ is the density of our 2DEG.

The quantum point contact [122, 129] is the basic building block of our mesoscopic circuits. We have three QPCs in our device (see Fig. 1.1). Their working principle is shown in Fig. A.2. The conductance of a QPC depends on the voltage applied on its two gates. The negatively higher the gate voltage, the narrower the constriction (and thus, the lower the conductance of the QPC). A measurement of the conductance of a QPC with respect to its two gates is shown in Fig. A.3.

This figure shows pronounced steps at multiples of $G_{K} \triangleq e^{2} / h$. At each step, one more electronic channel is transmitted through the QPC. The large plateaus at each step are actually due to the strong perpendicular magnetic field $(B \approx 2.7 \mathrm{~T})$ [22]. In this regime, the channels pass one-by-one. Hence, one can generalize the transmission $\tau$ to values larger than a unitary transmission when considering several channels (e.g. $\tau=3.14$ means that three channels are fully transmitted and a fourth has the intermediate transmission probability of 0.14 .) 


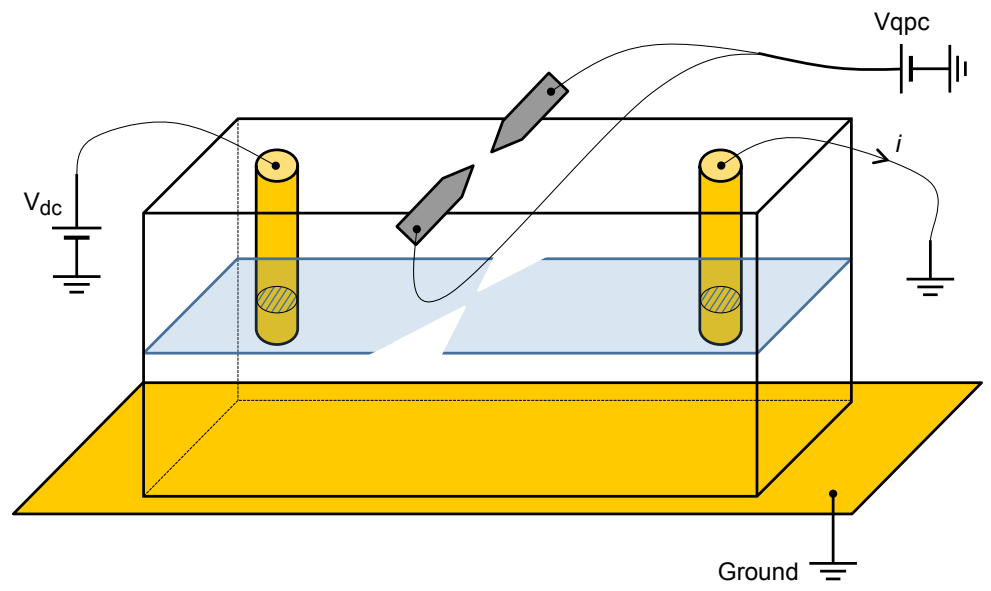

Figure A.2: Diagram of a Quantum point contact. The transparent cuboid represents the $(\mathrm{Al}) \mathrm{GaAs}$ heterostructure. A voltage $V_{\mathrm{qpc}}$ applied on the QPC gates generates an electric field between the metallic gates (in grey) and the ground (in gold). This field depletes the 2DEG under the gates. The constriction in the 2DEG is the QPC. The conductance $G=i / V_{\mathrm{dc}}$ of the $\mathrm{QPC}$ is measured using electrodes called 'ohmic contacts' (gold cylinders) that connect the 2DEG.

This conductance has been measured when the QPC was directly connected to a voltage source as drawn in Figs. A.2 and A.3. We will call this the intrinsic conductance ${ }^{4}$ of the QPC.

Note that the quantum of conductance $G_{K}=e^{2} / h \approx 1 / 25.8 \mathrm{k} \Omega$ is a universal value. The practical way to build the quantum conductor does not matter: its chemical composition, the density of the 2DEG, the geometry of the split gates, the voltage applied on the QPC gates, etc. Other examples of quantum coherent conductors include e.g. atomic-size contacts [109] or carbon nanotubes [41].

\section{A.2 Quantum transport of electricity}

In this section, we introduce some useful concepts on the quantum transport of electricity. We will discuss successively quantum Hall effect, dynamical Coulomb blockade and quantum shot noise. The theory will be described briefly and will be illustrated with measurements. The experimental data shown in the two last sections have been used to establish primary thermometers at $T \approx 6 \mathrm{mK}$ (see Appendix D, which reproduces an article where we have measured the lowest

\footnotetext{
${ }^{4}$ In presence of other components, the conductance of a coherent conductor is modified by the interactions with the environment.
} 

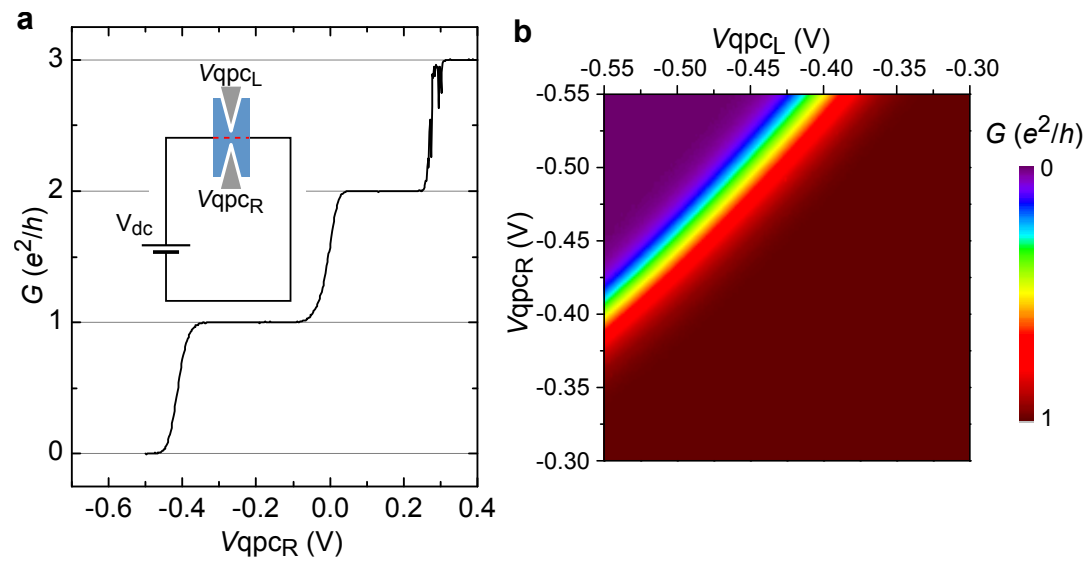

Figure A.3: Measurement of the conductance of a Quantum point contact. The intrinsic conductance of a QPC is measured with respect to the voltage applied on its two gates (left and right), in presence of a strong magnetic field (see text). a, The conductance of a QPC is measured as a function of the right gate, the left gate is fixed (to $V_{q p c_{\mathrm{L}}}=-0.55 \mathrm{~V}$ for this trace). b, Both gates are swept separately. A smooth step from 0 (in violet) to $G_{K}$ (in red) is observed.

electronic temperature in the world for a mesoscopic device of micrometer or nanometer scale with three different techniques).

\section{A.2.1 Quantum Hall effect}

The quantum Hall effect occurs when a 2DEG is subject to a strong perpendicular magnetic field [127]. The conductance of such a system acquires a universal value given by quantum of conductance $G_{K} \triangleq e^{2} / h$ times a filling factor $v$ that is either an integer or a fraction: $G=v G_{K}$. As explained by Laughlin in [68], this effect is an emergent phenomenon which cannot be deduced from first principles. In particular, despite a clean sample is required, the defects play a crucial since a sample which is invariant by translation cannot display quantum Hall effect [68]. The ground state of a fractional quantum Hall effect cannot be derived from a non-interacting electrons approach. This can lead to very complicated physics, for instance, the origin of the even-denominator filling factor $v=5 / 2$ remains an open question ([97] and references therein). In this thesis we consider only integer filling factors.

\section{Edge states and filling factor}

For the integer quantum Hall effect (IQHE), a semi-classical approach is relevant (for a derivation, see [47]). The energy of the electrons is quantized in Landau 
levels $E_{L}=(n+1 / 2) \hbar \omega_{C}$, where $\omega_{C}=e B / m$ is the cyclotron frequency (with $m$ the mass of the electron and $B$ the magnetic field). At a given Fermi energy $E_{F}$, only the levels with $E_{L}(n, B) \leq E_{F}$ will be filled and will participate to the electrical transport. This yields the quantum Hall plateaus of conductance versus the magnetic field shown in Fig. A.4a. Each mode will carry a quantum of conductance $G_{K}$ and will be located at the edge of the sample (see Fig. A.4p).

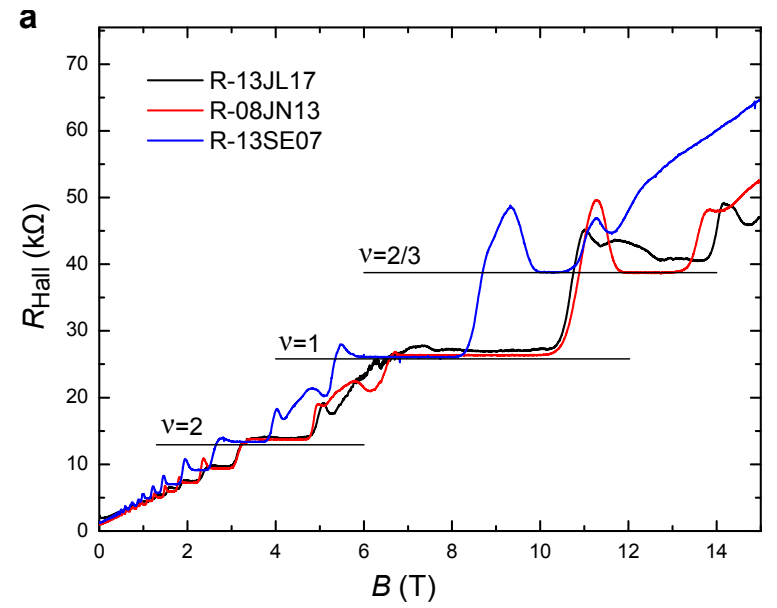

b

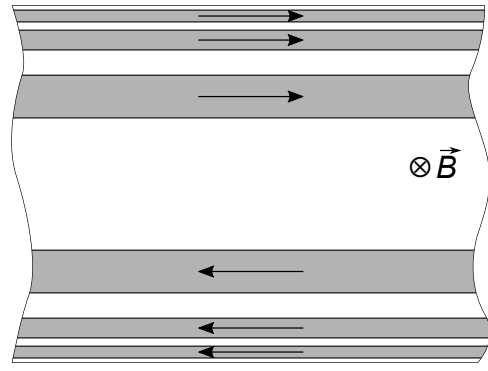

Figure A.4: Quantum Hall effect. a, The Hall resistivity is plotted versus the perpendicular magnetic field for several 2DEGs (three colors). The horizontal straight lines indicate the resistivity $R_{\text {Hall }}=v e^{2} / h$ expected for some filling factors. The mismatch comes from the resistance of the probes (a four-point probe method would be more accurate). The 2DEG used in this thesis is the '08JN13' (red trace). b, Representation of the chiral edge states for a $v=3$ integer filling factor.

\section{Percolation}

The "bulk" of the 2DEG is insulating, the current flows only along the chiral edge states. The width and the distance to edge of these edge states depend on the magnetic field [25]. When passing from a filling factor to the lower one, the innermost channel percolates as illustrated in Fig. A.5. This figure also shows that the screening of defects is better at higher filling factor, and, for a given filling factor, at the beginning of the quantum Hall plateau (low magnetic field). This can be observed when plotting the conductance of a QPC versus the voltage applied on its gates (as in Fig. A.3) for different magnetic fields. In practice, we determine the best operating conditions to perform our measurements with canonical QPCs. 
a
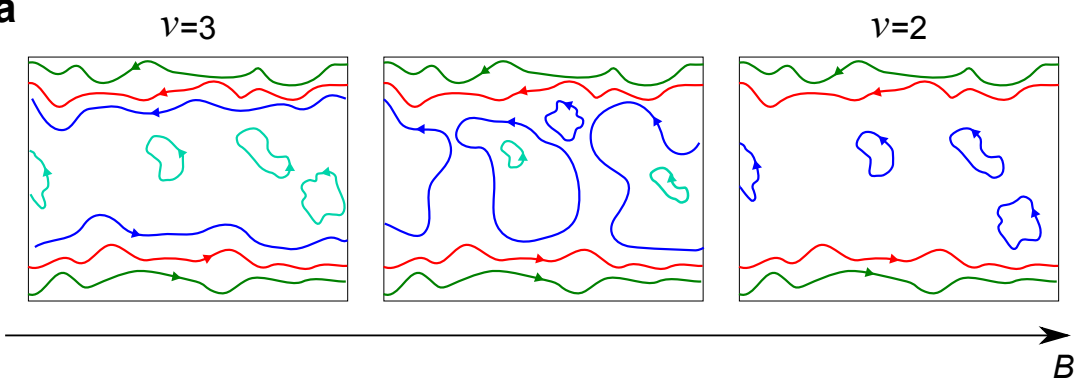

b
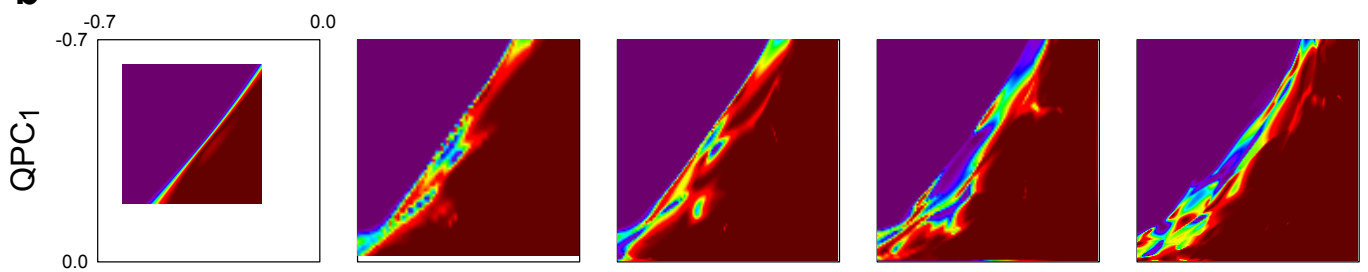

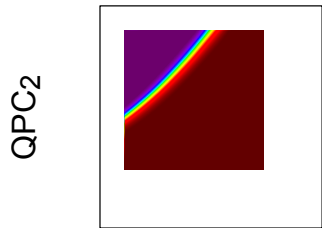

$B=2.7 \top$

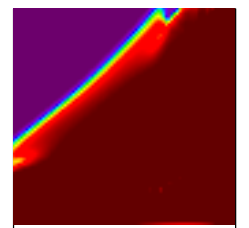

$B=3.8 T$

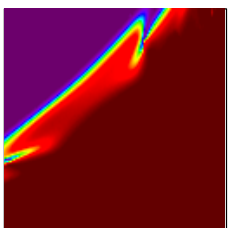

$B=4.0 \mathrm{~T}$

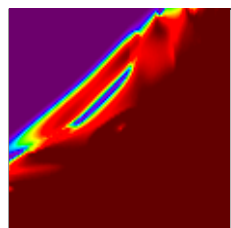

$B=4.4 \mathrm{~T}$

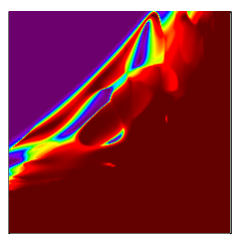

$B=4.8 \mathrm{~T}$

Figure A.5: Quantum Hall plateau. a, (adapted from [7]) The filling factor can be changed by tuning the magnetic field $B$. The edge states percolate in the intermediate region. b, The intrinsic conductance of $\mathrm{QPC}_{1}$ (first row) and $\mathrm{QPC}_{2}$ (second row) is plotted versus their two gate voltage $V_{\mathrm{qp} \mathrm{R}_{\mathrm{R}, \mathrm{L}}}$ for several magnetic field (see bottom line) but at the constant filling factor $v=3$. We use the same color code as in Fig. A.3b and the same range $V_{\mathrm{qpc}_{R, \mathrm{~L}}} \in[-0.7 \mathrm{~V}, 0 \mathrm{~V}]$ for all the graphs. The QPCs present a much more canonical shape in the beginning of the quantum Hall plateau (probably because the nearby defects are better screened by innermost channel).

\section{A.2.2 Dynamical coulomb blockade (DCB)}

Dynamical coulomb blockade modifies the conductance of a quantum conductor at low energy when it is embedded in a true circuit (and not directly connected to a voltage source, as in Fig. A.3). In general, the in situ conductance $G$ of a quantum conductor will be reduced compared to its intrinsic value $G_{\infty}=\tau G_{K} \geqslant G$ because the electrons that go through the conductor will dissipate some energy into the circuit [53]. This phenomenon is closely linked to the granularity of the charge that tunnels through the quantum conductor, and it will disappear at integer transmissions $\tau=1,2 \ldots$ [6]. 

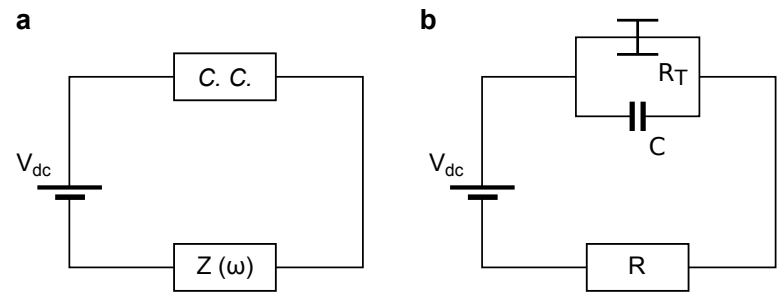

Figure A.6: A quantum coherent conductor in a circuit. a, The general problem of a coherent conductor (C.C.) in series with a impedance $Z(\omega)$. b, The theory is well-established in the case of low transmissions $\tau \ll 1$. Indeed the conductor can be separated in a pure tunnel element (of resistance $R_{T}$ ) in parallel with a capacitor $C$. We consider the case of a purely resistive impedance $Z(\omega)=R$.

The problem can be solved in the case of a short tunnel junction in series with any impedance $Z(\omega)$ (see the same review [53]). In practice, a short tunnel junction is always a parallel combination of a capacitor and pure tunnel element, as sketched in Fig. A.6. The geometry of the sample will set the capacitance $C$ and thus the charging energy $E_{C}=e^{2} /(2 C)$.

When dealing with resistive environments $Z(\omega)=R$, a charge that crosses the coherent conductor interacts with the electromagnetic modes of the environment. This interaction costs an energy that will change the transport properties of the coherent conductor at small voltage bias $V_{\mathrm{dc}}$ and low temperature. Indeed, in these conditions, the charge cannot pay the energy required to pass, it is then 'Coulomb blocked' and the differential conductance of the coherent conductor shows a dip when plotted versus the voltage bias $V_{\mathrm{dc}}$ (see Fig. A.7k).

\section{Theoretical predictions for a tunnel junction in series with a resistor}

At zero temperature, a perturbative theory gives the flowing expression for the conductance of a small and opaque tunnel junction in series with an arbitrary resistor $R$ at zero-temperature and with $e|V| \ll E_{C}$ (equation 113 in [53]):

$$
G(V)=\frac{G_{\infty}(\pi / \gamma)^{2 R / R_{K}}\left(1+2 R / R_{K}\right)}{\Gamma\left(2+2 R / R_{K}\right)}\left(R / R_{K} \frac{e|V|}{E_{C}}\right)^{2 R / R_{K}}
$$

where $G_{\infty} \triangleq 1 / R_{T}$ is the intrinsic conductance (as expected, we obtain $G=G_{\infty}$ for $R=0), \Gamma$ is the gamma function, $\gamma \approx \exp (0.5772)$ is the exponential of the Euler's constant.

There is also an expression of the conductance of the junction at zero bias and low temperature $k_{B} T \ll E_{C}[\overline{95]}$ : 


$$
G(T)=\frac{G_{\infty} \pi^{\left(1 / 2+3 R / R_{K}\right)} \Gamma\left(1+R / R_{K}\right)}{2 \Gamma\left(3 / 2+R / R_{K}\right)}\left(R / R_{K} \frac{k_{B} T}{E_{C}}\right)^{2 R / R_{K}}
$$

The resistance $R$ always appears in a ratio $R / R_{K}$. The strength of DCB depends on this ratio: (i) when $R \ll R_{K}, G(V) \sim G_{\infty}$, there is no Coulomb blockade (ii) when $R \gg R_{K}$, we are in the static Coulomb blockade regime 5 , the current is completely blocked unless the electrons have the charging energy $e|V|>E_{C}$ (iii) the ideal case to observe a fully developed DCB is when $R \sim R_{K}$.

From the two previous expressions, we can extract the experimental (absolute) temperature of a DCB measurement in the tunnel regime in the case (iii) with $R=R_{K} / 2$. This measurement can be used to perform a primary 6 thermometry.

\section{Experimental DCB measurement}

We repeat the measurement twice, once with $\mathrm{QPC}_{1}$ and once with $\mathrm{QPC}_{2}$. To implement the circuit shown in Fig. A.6b, we use $\mathrm{QPC}_{i}$ as a tunnel junction, and we set the opposite characterization gate to $\tau_{s w_{j}}=2$. This switch will behave as a classical resistor 7 with a resistance $R=R_{K} / 2$.

At low temperature, we measure the conductance versus the voltage bias $V_{\mathrm{dc}}$. The conductance shows a dip at zero bias (see Fig. A.7). The deeper the dip, the lower the temperature. At $R=R_{K} / 2$, the expressions Eqs. A.4) and (A.5) of the conductance are linear both in $V$ (see the red dashed lines in Fig. A.7k) and $T$ :

$$
\left\{\begin{array}{l}
G(V)=\alpha \frac{G_{\infty}}{E_{C} / k_{B}} \times V \\
G(T)=\beta \frac{G_{\infty}}{E_{C} / k_{B}} \times T
\end{array}\right.
$$

where $\alpha=\frac{\pi e}{2 \gamma} / k_{B} \approx 10.23 \times 10^{3}$ and $\beta=\frac{\pi^{5 / 2}}{8} \approx 2.18$ are constants. To be more accurate 8 , we can extract $E_{C} \approx 25 \mu \mathrm{eV} \approx 290 \mathrm{mK}$ from the height of the Coulomb diamonds shown in Fig. A.7. The explanations on Coulomb diamonds will be given in Appendix C.2. From this numerical value of $E_{C}$, we can confirm that these DCB experiments were actually done in the tunnel regime: $G_{1 \infty} \approx 0.12 G_{K}$ and $G_{2 \infty} \approx 0.13 G_{K} \ll G_{K}$. Then we use a finite temperature theory of the tunnel regime proposed by [57]. Their calculations have been plotted (as red solid lines) in Fig. A.7 $\mathrm{abc}$, where we have used $E_{C}=25 \mu \mathrm{eV}$ and

${ }^{5}$ An implementation of this limit will be discussed in Appendix C, where we consider the single electron transistor (SET).

${ }^{6}$ Such a thermometer does not require any calibration with a reference thermometer.

${ }^{7}$ An experiment reported in this thesis (in Fig. 4.10 shows that this is not true on the first plateau $R=R_{K}$.

${ }^{8}$ The temperature extracted will remain a primary thermometry. 

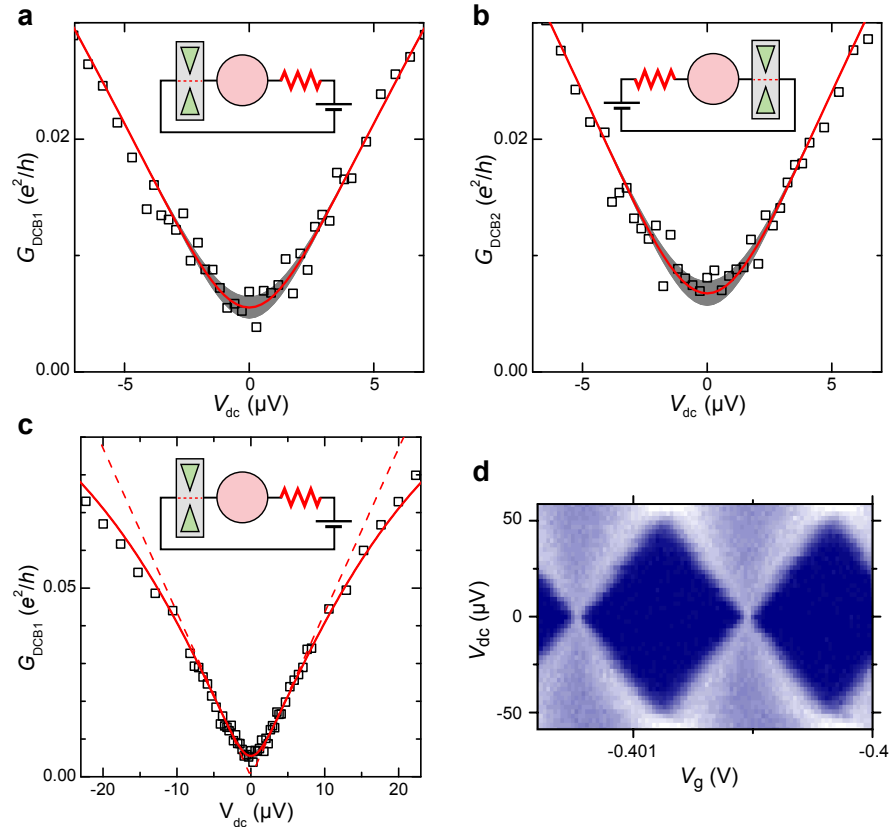

Figure A.7: Dynamical coulomb blockade measurement. This measurement is done at $T \approx 6 \mathrm{mK}$ in the tunnel regime, with a resistive environment $R=R_{K} / 2$. a and $\mathbf{b}$, With a resistor in series, the conductance $G_{\mathrm{DCB}}$ of a QPC is reduced at zero bias $V_{\mathrm{dc}}$. The red line corresponds to the finite temperature theory for a tunnel junction at $T_{\mathrm{DCB} 1}=(6.0 \pm 1.0) \mathrm{mK}$ for $\mathrm{QPC}_{1}$ and $T_{\mathrm{DCB} 2}=(6.5 \pm 1.0) \mathrm{mK}$ for $\mathrm{QPC}_{2}$ (the grey shade shows the uncertainty $\pm 1 \mathrm{mK}$ ) c, Same as a, but displayed on a wider range in $V_{\mathrm{dc}}$. d, Coulomb diamond, its height gives the charging energy $E_{C} \approx 25 \mu \mathrm{V}$ (explanations given later, in Appendix C.2).

$T_{\mathrm{DCB} 1}=(6.0 \pm 1.0) \mathrm{mK}$ for $\mathrm{QPC}_{1}$ or $T_{\mathrm{DCB} 2}=(6.5 \pm 1.0) \mathrm{mK}$ for $\mathrm{QPC}_{2}$ to fit the data using $G_{\infty}$ as a fitting parameter, the gray shade shows the uncertainty $\pm 1 \mathrm{mK}$.

We have seen that we are able to measure the temperature of a dynamical Coulomb blockade measurement in the tunnel regime with an environment $R=$ $R_{K} / 2$. This phenomenon is due to the granularity of the charge that flows through the tunnel junction made by a QPC. This granularity can be measured in the fluctuations of the current across the QPC. We will discuss this point in the next section.

Here we have discussed DCB in the tunnel regime using QPCs. This effect can also be explored in the strong coupling regime [99] or using atomic junction has shown by the Quantronics group (see [31], or the thesis of R. Cron [32]). 


\section{A.2.3 Quantum shot noise}

So far, we have only discussed the average current. But, additional informations are contained in the fluctuations of current. We are going to measure these fluctuations to get a temperature measurement. In practice, we are able to measure the power spectrum of the current fluctuations $S_{I}$ at zero frequency. This quantity can be computed using a scattering approach for a short coherent conductor connecting two terminals with a transmission $\tau$ (equation 62 in [16]):

$$
S_{I}=\frac{e^{2}}{h}\left[4 k_{B} T \tau^{2}+2 e V_{\mathrm{dc}} \tau(1-\tau) \times \operatorname{coth}\left(\frac{e V_{\mathrm{dc}}}{2 k_{B} T}\right)\right]
$$

in this expression, the transmission $\tau(E) \approx \tau$ is assumed independent of the energy for $E \lesssim e\left|V_{\mathrm{dc}}\right|, k_{B} T$. The noise is maximal when $\tau=1 / 2$. At small voltage bias $e V_{\mathrm{dc}} \ll k_{B} T$, the noise corresponds to the thermal (Nyquist-Johnson) noise for a classical resistor $S_{I}=4 k_{B} T G_{\infty}$ where $G_{\infty} \triangleq \tau e^{2} / h$ is the intrinsid 9 conductance of the quantum conductor. This is also the case when $\tau=1$, since the second term vanishes and $G_{\infty}=e^{2} / h$.
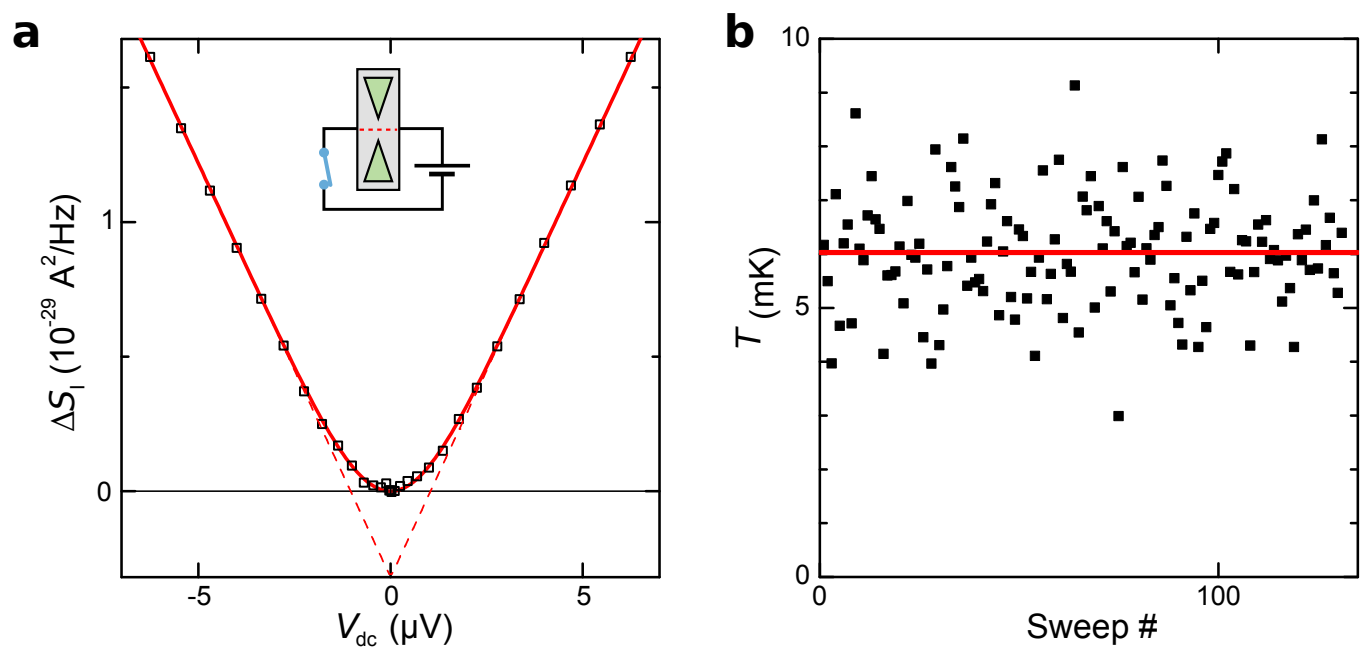

Figure A.8: Primary thermometry using quantum shot noise. a, Power spectral density of current fluctuations in excess $\Delta S_{I} \triangleq S_{I}-S_{I}\left(V_{\mathrm{dc}}=0\right)$ versus voltage bias $V_{\mathrm{dc}}$, for a quantum conductor at $\tau \approx 0.55$ directly connected to a voltage source (see the inset), and averaged on 131 sweeps. Solid red line shows a fit of the averaged data. We obtain $T=(6.0 \pm 0.1) \mathrm{mK}$. The dashed lines correspond to zero temperature. $\mathbf{b}$, Temperature obtained to fit the data of each successive sweep of $V_{\mathrm{dc}}$.

\footnotetext{
${ }^{9}$ In this experiment, the quantum conductor is directly connected to a voltage source (see the inset of Fig. A.8a).
} 
In Fig. A.8, we set $\tau \approx 0.5$ and we use Eq. A.7) to extract the temperature $T$. This is a primary thermometry as we are able to characterize the other parameters $\left(V_{\mathrm{dc}}, \tau\right)$. The temperature $T=(6.0 \pm 0.1) \mathrm{mK}$ is essentially given by the curvature of the excess noise $\Delta S_{I}$ at low voltage bias. The accuracy on the measurement can be significantly enhanced by statistically averaging the measure on many sweeps (or, in principle equivalently, by increasing the integration time). The precision is then limited by the stability of the experimental setup on long time scales (the Fig. A.8 requires $\sim 45 \mathrm{~h}$ of acquisition). 


\section{Appendix B}

\section{Sample description and characterization}

Figure B.1 shows a colored micrograph of the sample with the name of each element. The elements outside the image are of macroscopic size. There are two types of connections to the outside world: (i) the white circles (ii) the black circle. The black circle is a usual connection: a conductive wire touches the element on the surface. The white circles are ohmic contacts: an alloy of Au-Ni-Ge is diffused to connect the underlying 2DEG by a thermal annealing. The white circles are thus used for injecting or measuring current while the black circles are used to shape the circuit.

The sample is subjected to a strong magnetic field giving rise to IQHE. In this regime, the current is flowing along chiral edge states. In Fig. B.1, only the outermost channel is shown, and not everywhere in order to lighten the figure. Due to the chiral edge states of the quantum Hall effect, the injection and the measurement points are not shot-circuited and one can for instance measure the signal reflected back from a QPC.

This chapter is divided into a section about the gates on the surface (QPCs and SWs gates) and a section dealing with the central micron-sized ohmic contact. The first section explains how we can implement the different circuits studied in this thesis and it shows our mastery of the quantum conductor embedded in these circuits. The second section shows the good quality of the central ohmic contact which ensures the circuit we implement in practice to be a 'perfect quantum simulation' [85] of the explored phenomena. 


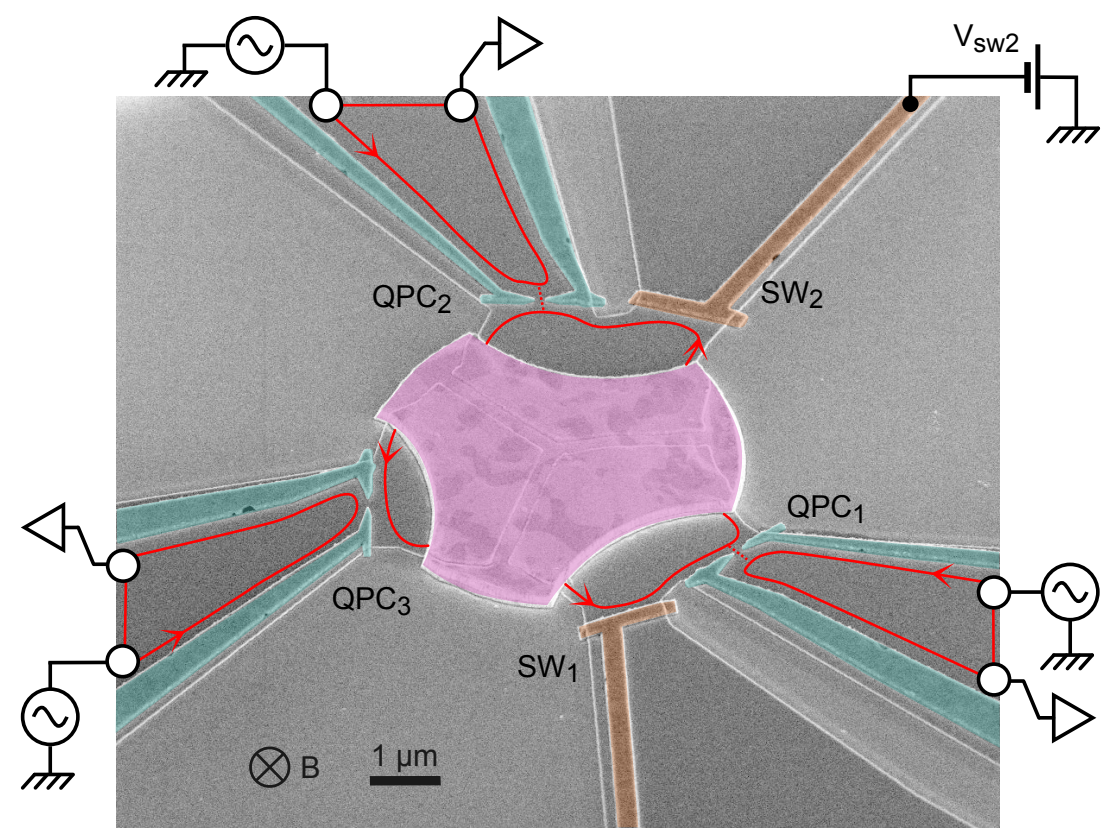

Figure B.1: Colored micrograph of the sample with measurement schematics. This figures shows the central ohmic contact (in purple) that redistributes the current injected by the a.c. voltage sources (out of the image) into the chiral edge states of the IQHE (red lines) through larger ohmic contacts (white circles) not shown in the picture. The low frequency signal is measured using Lock-in amplifiers (triangles). This sample includes three QPCs (in cyan) and two switch gates (in orange) used for characterization. The value of the transmission of the QPC and the state of the switch is controlled by the voltage sources that connect (black circles) theses gates placed on the surface.

\section{B.1 Surface gates}

The principle of field effects to change the conductance of the constriction of the 2DEG below surface gates has been explained in Fig. A.2 for a QPC. This principle works as well for the switch gates (SWs) used for characterization.

\section{B.1.1 Switch gates and environment}

The switch gates are not used at intermediate transmission because the region right under the gate is generally not clean (one can see the sharp resonances in the gray shaded areas of Fig. B.2). Their geometry is optimized to change the transmission (integer) values quickly, without applying large voltages $V_{\mathrm{sw}}$.

The main purpose of these switch gates is the characterization of the intrinsic 


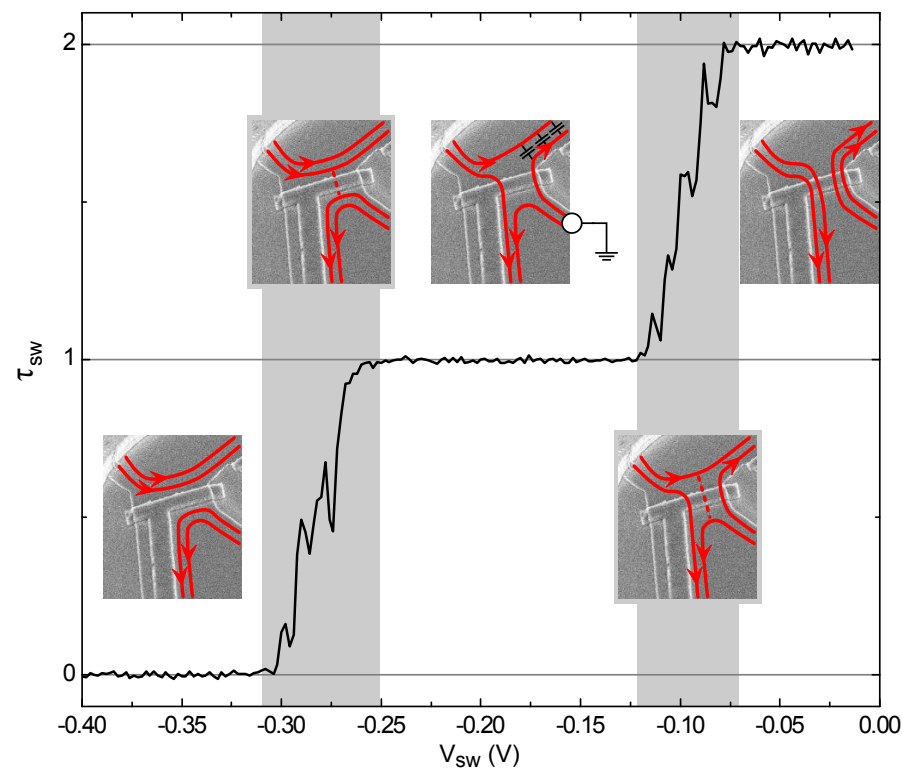

Figure B.2: A switch gate at work. This figure shows the successive opening of electronic channels by a switch when we sweep the voltage $V_{s w}$. The insets show the back-scattering of the channels in (the gray shaded areas) and the ballistically transmitted channels (areas without background). The central inset shows two additional information: the switch 'injects' grounded channels and there is a (negligible) capacitive coupling between the edge states.

conductance of the QPCs 1 . The switches have an injection port, but it is not used to inject current, the edge states lines comes from the ground. When the switch let the current pass, it short-circuits its QPC to the ground. The equivalent circuit is given in Fig. B.3a.

Being in the IQHE regime is not mandatory for the switch gates to work. Indeed, the environment of the QPC will be short-circuited once its lateral switch gate is fully transmitting all the electronic channels. In the IQHE regime, one will measure the intrinsic transmission $2^{2} \tau \leqslant n_{\text {channels }}$ of a QPC (where $n_{\text {channels }}$ is the maximum number of channels opened by the QPC for a given characterization) if its lateral switch let pass at least $n_{\text {channels }} \leqslant v$.

There might be an interaction (sketched with distributed capacitors in the central inset of Fig. B.2) between the edge states that put some signal in the strict case $n_{\text {channels }}<v$, but this is completely negligible on the small distance between a switch and its QPC. In practice all our experiments are done with $\tau \leqslant 1$, and we open at least the switches on $n_{\text {channels }}=2$.

${ }^{1}$ There is no switch gate for the third QPC.

${ }^{2}$ As explained when discussing Fig. A.3 in the IQHE regime, we can define transmissions larger than $\tau=1$ when considering the transmission of more than a single edge channel. 


\section{B.1.2 Characterization and measurement circuitry}

We have seen that we are able to change the environment of a QPC by changing the state of the switches. Changing the voltage on the switches gate also have an electrostatic influence on the size of the constrictions of the QPCs. This point will be discussed in the next subsection. Here we discuss different configurations of the switches and the QPCs.
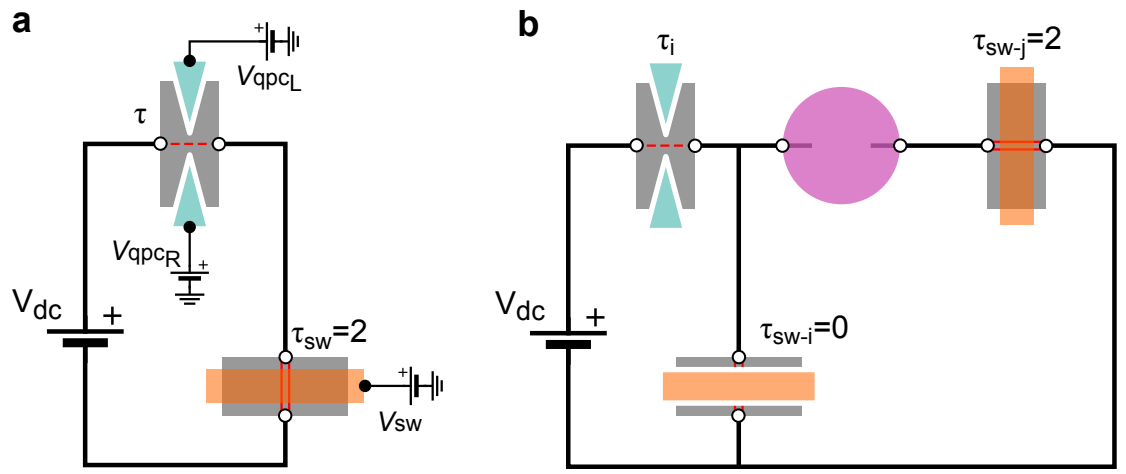

Figure B.3: Circuits used for characterization. The shape of the 2DEG (in gray) can be modified thanks to the surface gates (in cyan and orange). Theses gates are connected (black circles) to voltage sources. The connection of the 2DEG with the circuit is achieved with ohmic contacts (white circles). a, Circuit for the measurement of the intrinsic conductance $G_{\infty}=\tau G_{K}$. The switch let pass two channels and $\tau \leqslant 1$. The switch is in its closed state, it can be replaced by black classical wire (as in the inset of Fig. A.3 $)$ ). b, In series with an element, the conductance of the $\mathrm{QPC}_{i}$ will be modified $G_{i} \neq \tau_{i} G_{K}$. Here the environment of $\mathrm{QPC}_{i}$ is a resistor $R=R_{K} / 2$ (made with the switch $\mathrm{SW}_{j}$ of transmission $\tau_{s w_{j}}=2$ ). The branch containing $\mathrm{SW}_{i}$ can be erased as it is placed in its open state. This is a typical circuit for Dynamical coulomb blockade.

The switch gates are used to implement two characterization circuits. We can use them to short-circuit the environment (Fig. B.3 a) or to make a wellcontrolled resistive environment (Fig. B.3p). The first circuit measures the intrinsic transmission $\tau$ of a QPC while the second one probes the conductance in presence of DCB.

The circuit actually studied to get the main results reported in this thesis is given in Fig. B.4. The switches were used for characterization only, they are set to $\tau_{s w_{1}}=\tau_{s w_{2}}=0$ during the experiments. However we use $V_{s w_{2}}$ as a plunger gate. This gate is swept on a very small range (generally in $[-0.395 \mathrm{~V},-0.405 \mathrm{~V}]$ ), and with a good margin to keep $\tau_{s w_{2}}=0$ (see Fig. B.2). 


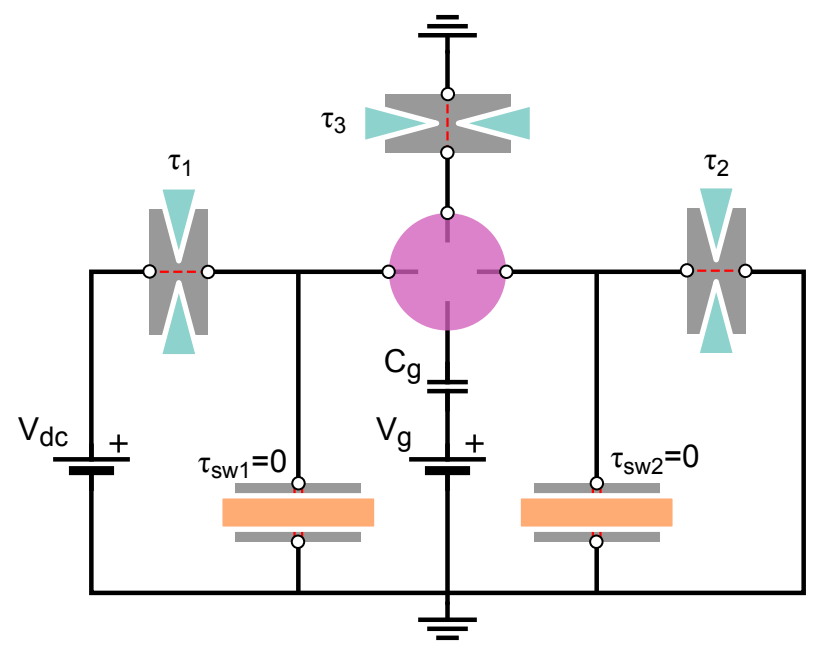

Figure B.4: Circuit that produced the results reported in this thesis. The branches containing the switches can be erased as both are in opened position. In the charge quantization experiment $\tau_{3}=0$, its branch can be suppressed too. We always have $\tau_{1}, \tau_{2}, \tau_{3} \leqslant 1$. When $\tau_{1}, \tau_{2}, \tau_{3} \ll 1$, the charge on the island (in purple) is quantized because it is almost isolated from the circuit. This charge state can be tuned using the voltage $V_{g}$ applied on a surface gate (e.g. $V_{s w_{2}}$ ) of an element at $\tau=0$ (not to modify its transmission).

\section{B.1.3 Quantum point contacts transmission and characteriza- tion}

Let us discuss how we implement a QPC with a well characterized transmission $\tau$. First, this transmission has an energy dependence and its variation $\Delta \tau$ on the range of explored temperatures has to be minimized to guaranty that we were dealing with the same object at the different temperatures. Second, we will play with several QPCs; changing the voltages on the gate of a QPC influences the size of the constriction of the other QPCs and therefore their transmission: these crosstalks should be fully characterized. Third, we use the switches to measure the intrinsic transmissions, but we switch their state to perform the real experiment, the crosstalk between a QPC and its lateral switch gate should also be characterized. These two corrections will be discussed in the next subsection.

Quantum point contacts (QPCs) give us access to the full range of transmission $\tau \in[0,1]$. For the purpose of our experiments, we will pick up a dozen of values in this range. We can check the energy dependence of these selected values of transmission by applying a voltage bias. Figure B.5 shows the transmission of a QPC and the values picked up for the two-channel Kondo experiment (published [52], but not shown in this thesis). The inset shows the energy dependence $\Delta \tau / \tau$ for three of these values versus the voltage bias (the highest temperature 
in this experiment was $T_{\max } \approx 151 \mathrm{mK} \approx 13 \mu \mathrm{eV} / k_{B}$ and the voltage range is $[-50,+50] \mu \mathrm{V})$.

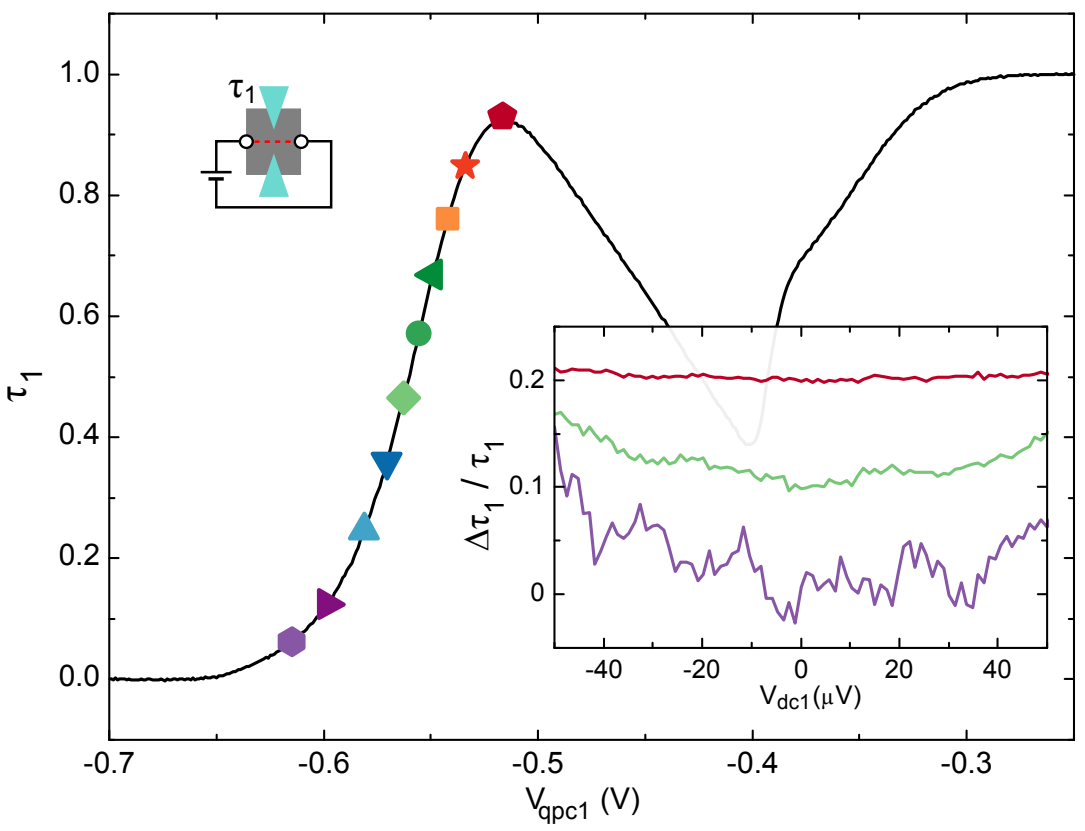

Figure B.5: Energy dependence of the intrinsic transmission $\tau$. The transmission $\tau_{1}$ of $\mathrm{QPC}_{1}$ is measured as a function of the voltage $V_{\mathrm{qpc}_{1}}$. The step from $\tau_{1}=0$ to 1 is marked by a broad resonance around $V_{\mathrm{qpc}_{1}}=-0.4 \mathrm{~V}$, but we pick up our points (symbols) away from it. The energy dependence is shown in the inset for three selected transmissions, where the difference relatively to $V_{\mathrm{dc}}=0$ is plotted (with an offset for clarity).

Let us verify that our QPCs are generic and interchangeable. A single-channel quantum conductor (as our QPCs in the IQHE regime) is fully characterized by its transmission $\tau$. In Fig. B.6, we have set the three QPCs in turn in the same DCB configuration ( $T=18 \mathrm{mK}$ and a resistive environment of $R_{K} / 2$ as shown in Fig. B.6 a). We have tuned each QPC to a given conductance at zero voltage bias, and we observe in Fig. B.6p that all the DCB curves develop equally (up to some energy dependence) for all the QPCs.

The value of the transmission we have used in Chapters 3 and 4 are obtained from the conductance in this DCB configuration, at large voltage bias (in the gray shaded areas of Fig. B.6p). The conductance is not the same for positive and negative large bias because of the energy dependence of the QPCs. This limits the accuracy for the determination of a single transmission. The $\Delta \tau$ associated to each transmission $\tau$ in the table of Fig. B.6 takes into account both the positive/negative bias and $\mathrm{QPC}_{1,2,3}$ scatter (six values for each $\tau$ ), to get an estimation of the accuracy 
on $\tau$.
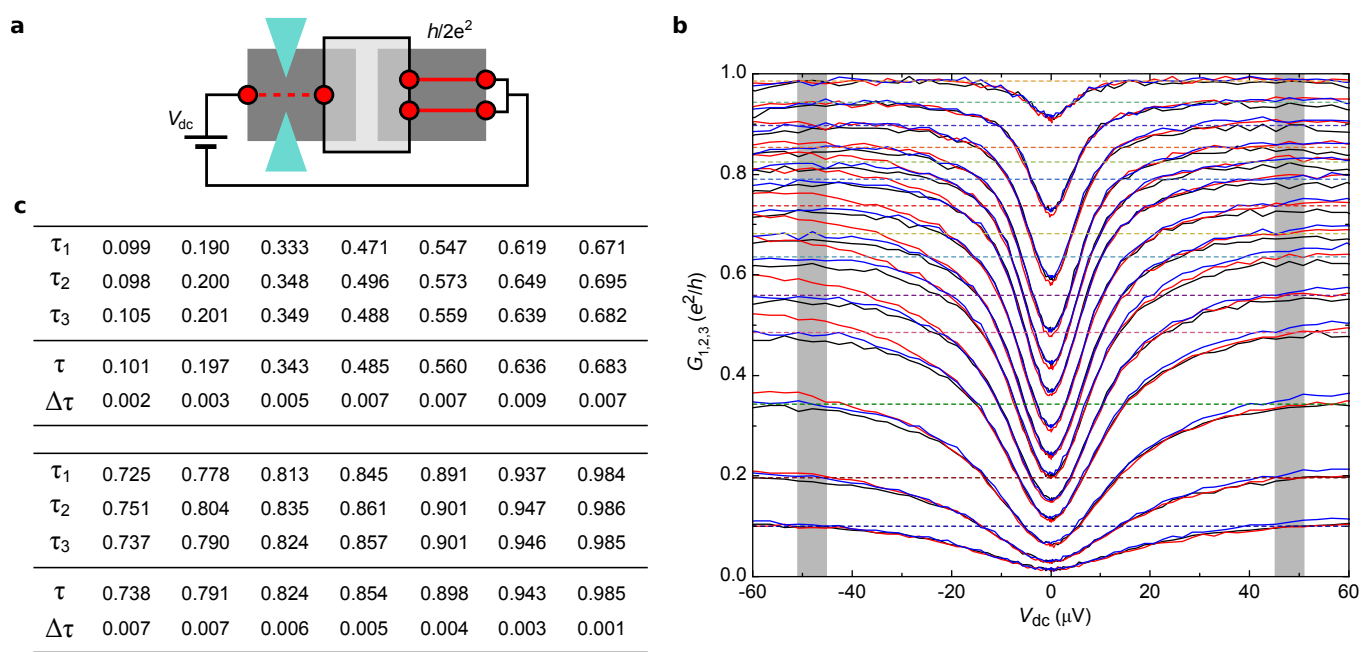

Figure B.6: Equivalence of QPC in presence of DCB and accuracy on the transmission $\tau$. a, Schematic circuit used to characterize the transmission probabilities of the QPCs. A lateral characterization gate is used to implement a serial resistance of $R=R_{K} / 2$. b, The conductance $G_{1,2,3}^{\mathrm{DCB}}$ of $\mathrm{QPC}_{1}, \mathrm{QPC}_{2}$ and $\mathrm{QPC}_{3}$ is measured (one after another) in presence of DCB at $T=18 \mathrm{mK}$ with the same electromagnetic environment $\left(R=R_{K} / 2\right.$ and $\left.E_{C} \approx 25 \mu \mathrm{eV}\right)$. We see that the three QPCs are interchangeable up to $e\left|V_{\mathrm{dc}}\right| \approx E_{C}$. The intrinsic conductance can be extracted from the conductance at large d.c. voltage bias (in the $\pm[45,51] \mu \mathrm{V}$ gray shaded areas) to obtain the transmission $\tau_{1,2,3}=G_{1,2,3}^{\mathrm{DCB}}\left(e\left|V_{\mathrm{dc}}\right| \gg E_{C}\right) / G_{K}$. c, These individual transmission probabilities $\tau_{1,2,3}$ are averaged (from six values corresponding to positive/negative bias and $\mathrm{QPC}_{1,2,3}$ ) to give $\tau$ with a standard error on the mean value $\Delta \tau$.

\section{B.1.4 Capacitive crosstalk corrections}

Changing the voltage on a surface gate has an electrostatic capacitive influence on all the constrictions (QPCs) of the sample. This electrostatic effect depends basically on the distance between the two objects we consider. It can be characterized and corrected by applying a correction to each QPCs gates to compensate the change on a given surface gate.

These corrections are relatively small but they can have a certain impact on the sensitive measurement reported in this thesis. We first discuss the case of distant gates and then treat the crosstalk of a switch gate on its lateral QPC. 


\section{Influence on a distant gate}

The influence on the change on the voltage gates of a $\mathrm{QPC}_{i}$ onto the transmission of a $\mathrm{QPC}_{j}$ is maximal when $\tau_{j} \sim 0.5$ because the slope $\partial \tau / \partial V_{\mathrm{qpc}}$ is maximal around this value. To be more accurate, we characterize the crosstalk $\mathrm{QPC}_{i} \rightsquigarrow \mathrm{QPC}_{j}$ in two steps. First, we try to adjust the coefficient $\alpha$ such that the variation, $\delta \tau_{j}$, induced on the transmission of $\mathrm{QPC}_{j}$ by a step $\delta V_{\mathrm{qpc}_{i}}$ is compensated by $\delta V_{\mathrm{qpc}_{j}}=\alpha \delta V_{\mathrm{qpc}_{i}}$ so that we can sweep $V_{\mathrm{qpc}_{i}}$ (on the range $\tau_{i}: 0 \rightarrow 1$ ) and keep $\tau_{j}$ constant. Second, we determine the absolute shift $\Delta V_{\mathrm{qpc}_{j}}$ measured on $\tau_{j}$ when we pass from $\tau_{i}=0$ to $\tau_{i}=\tau_{i}^{\text {ref }}$ (where $\tau^{\text {ref }}$ is a picked up value close to half transmission for the QPCs and $\tau_{s w}^{\mathrm{ref}}=2$ when calibrating the influence of a SW gate onto a QPC).

These calibrations of the crosstalks between the surface gates are done using different configuration of the switch gates. A full set of calibration with their corresponding configuration of all the surface gates is given in Table B.1. There are several remarks to do here: (i) the crosstalks $\mathrm{QPC}_{i} \rightsquigarrow \mathrm{QPC}_{j}$ and $\mathrm{QPC}_{i}$ in $\mathrm{QPC}_{j}$ are not equal (despite the electrostatic origin of this effect) in particular because the compensation $\delta V_{\mathrm{qpc}_{j}}=\alpha \delta V_{\mathrm{qpc}_{i}}$ depends on $\partial \tau_{j} / \partial V_{\mathrm{qpc}_{j}}$, (ii) $\mathrm{QPC}_{3}$ has no switch and (iii) these effects are small and only the first order is considered (crosstalks of crosstalks are neglected). The point (ii) is not a problem in principle since calibrating a crosstalk $\mathrm{QPC}_{i} \rightsquigarrow \mathrm{QPC}_{3}$ is possible even if the $\mathrm{QPC}_{3}$ is not in an 'intrinsic configuration'. Indeed, its environment will be fixed to $R_{K} / 2$ thanks to $\tau_{s w_{i}}=2$ (whatever $\tau_{i} \leqslant 2$ ). In practice we have noticed an effect of $\tau_{i}: 0 \rightarrow 1$ when we try to adjust this correction, but we can compare with $\mathrm{QPC}_{1}$ and $\mathrm{QPC}_{2}$ (which show the similar slight change of environment when they are in 'DCB configuration').

\section{Influence of a switch gate on its lateral QPC}

This characterization has been used to extract the transmission $\tau_{i}$ of a $\mathrm{QPC}_{i}$ (with $i=L$ or $R$ ) in the charge quantization experiment. It consists in taking a trace of $\mathrm{QPC}_{i}$ for the two configurations of $\mathrm{SW}_{i}$ : 'open' and 'closed' (see Fig. B.7b). In the open position, the $\mathrm{QPC}_{i}$ is sensitive to its electronic environment and one should apply a large d.c. voltage bias $\left(e V \gg E_{C}\right.$, to avoid any renormalization of the conductance due to dynamical Coulomb blockade). Note that the voltage drop across the $\mathrm{QPC}_{i}$ in the red inset of Fig. B.7p depends on its transmission as $\tau_{L} /\left(\tau_{L}+\tau_{R}\right) V$, one should then focus on $\tau_{R} \ll 1$ (to be in the same conditions as in the blue inset where the environment is short-circuited).

The spacing $\Delta V_{R}^{\mathrm{ct}}$ between the two traces in Fig. B.7p is directly the correction to apply on the right QPC voltage gate $V_{\mathrm{qpc}_{R}}$ when switching the state of its lateral switch. This spacing $\Delta V_{R}^{\mathrm{ct}}$ is evaluated by focusing on small $G_{\mathrm{qpc}_{R}}$ in order to 


\begin{tabular}{|c|c|c|c|c|c|c|c|c|c|}
\hline \multirow{6}{*}{ 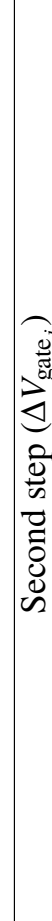 } & $\Leftrightarrow$ & 0 & $\mid \begin{array}{l}\overrightarrow{2} \\
2 \\
\infty \\
0\end{array}$ & 0 & $\begin{array}{l}\overrightarrow{2} \\
-m \\
\infty \\
0\end{array}$ & $\begin{array}{l}\vec{\uparrow} \\
0\end{array}$ & $\begin{array}{l}\vec{\uparrow} \\
0\end{array}$ & 0 & 0 \\
\hline & $N$ & $\begin{array}{l}\vec{\uparrow} \\
0\end{array}$ & $\begin{array}{l}- \\
\uparrow \\
0\end{array}$ & 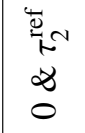 & 0 & 0 & 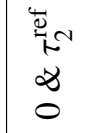 & $\vec{\uparrow}$ & \\
\hline & $F$ & 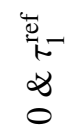 & 0 & $\begin{array}{l}\vec{\uparrow} \\
0\end{array}$ & $\begin{array}{l}\vec{\uparrow} \\
0\end{array}$ & 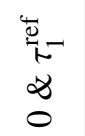 & 0 & & $\vec{\uparrow}$ \\
\hline & 产 & $\underbrace{N O}$ & $N$ & $\underbrace{O N}$ & 0 & $\underbrace{N O}$ & $\underbrace{N \sim}$ & & $\begin{array}{lll}N & 2 \\
\infty & 2 \\
0 & 0\end{array}$ \\
\hline & 恿 & $\underbrace{O N}$ & 0 & $\underbrace{N O}$ & $N$ & $\underbrace{\mathrm{NN}}$ & $\underbrace{N O}$ & $\begin{array}{l}N \\
\infty \\
0\end{array}$ & 0 \\
\hline & 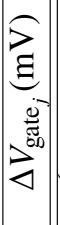 & $\begin{array}{rl}-3 & - \\
0 & 0 \\
+1 & +1 \\
m & 0 \\
0 & 0 \\
0\end{array}$ & $\begin{array}{c}- \\
0 \\
+1 \\
0 \\
-\end{array}$ & 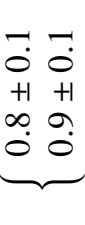 & $\begin{array}{c}- \\
0 \\
0 \\
+1 \\
n \\
-1 \\
-\end{array}$ & 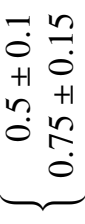 & $\underbrace{n} \begin{array}{ll}n & n \\
0 & 0 \\
+1 & 0 \\
+1 & +1 \\
0 & 0 \\
0 & 0 \\
n & n \\
n\end{array}$ & 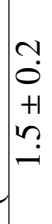 & 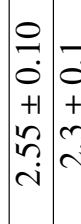 \\
\hline \multirow{7}{*}{ 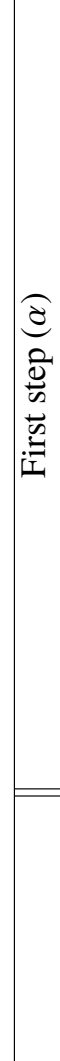 } & $\Leftrightarrow$ & 0 & 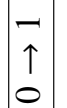 & 0 & $\begin{array}{l}-\vec{\uparrow} \\
0\end{array}$ & $\mathrm{~g}$ & $\stackrel{m}{r}$ & & \\
\hline & $\mathbb{N}$ & "ָّ & 㱐 & $\begin{array}{l}\vec{\uparrow} \\
0\end{array}$ & 0 & 0 & $\begin{array}{l}\widehat{\uparrow} \\
0\end{array}$ & & \\
\hline & $F$ & $\begin{array}{c}\vec{\uparrow} \\
0\end{array}$ & 0 & 党 & 莺- & $\begin{array}{l}\vec{\uparrow} \\
0\end{array}$ & 0 & & \\
\hline & 恿 & $N$ & $\sim$ & $N$ & 0 & 0 & $N$ & & \\
\hline & $\mid$\begin{tabular}{|c||}
$\overrightarrow{3}$ \\
咅
\end{tabular} & $N$ & 0 & $N$ & $N$ & $N$ & 0 & & \\
\hline & $\mid$ & 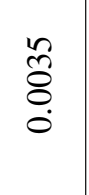 & $\overrightarrow{\bar{\sigma}}$ & $\begin{array}{l}0 \\
8 \\
0 \\
0\end{array}$ & $\underset{0}{1}$ & $\begin{array}{l}n \\
\delta \\
0 \\
0\end{array}$ & $\stackrel{\text { â }}{0}$ & & \\
\hline & 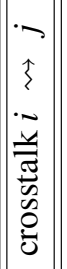 & $\begin{array}{c}U^{N} \\
\tilde{\sigma} \\
\hat{z} \\
\dot{\sigma} \\
\bar{\sigma}\end{array}$ & 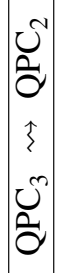 & $\begin{array}{l}v \\
\vdots \\
o \\
\hat{\sigma} \\
v \\
\vdots \\
\vdots\end{array}$ & 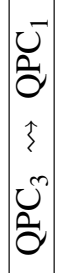 & $\begin{array}{l}\dot{v} \\
\tilde{\sigma} \\
\hat{\xi} \\
\dot{\sigma} \\
\dot{\sigma}\end{array}$ & $\begin{array}{l}\hat{\theta} \\
\hat{\sigma} \\
\hat{z} \\
\tilde{\sigma} \\
\dot{\sigma}\end{array}$ & & 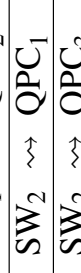 \\
\hline
\end{tabular}

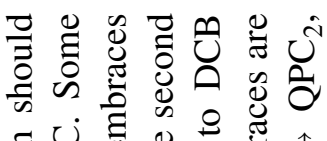
ธี

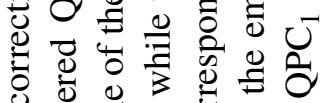

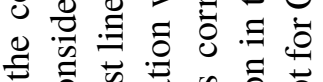

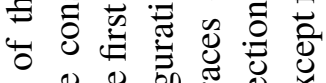
БÐ 巳

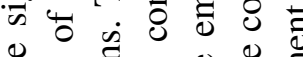
E 00.

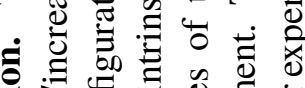

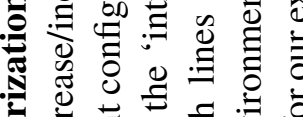

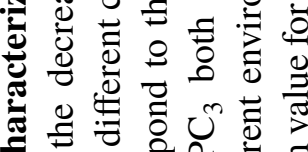

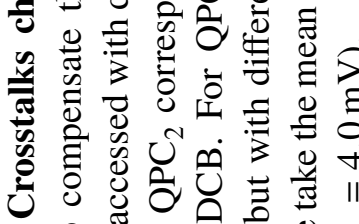

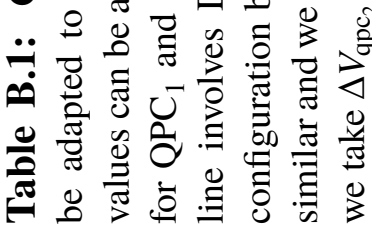



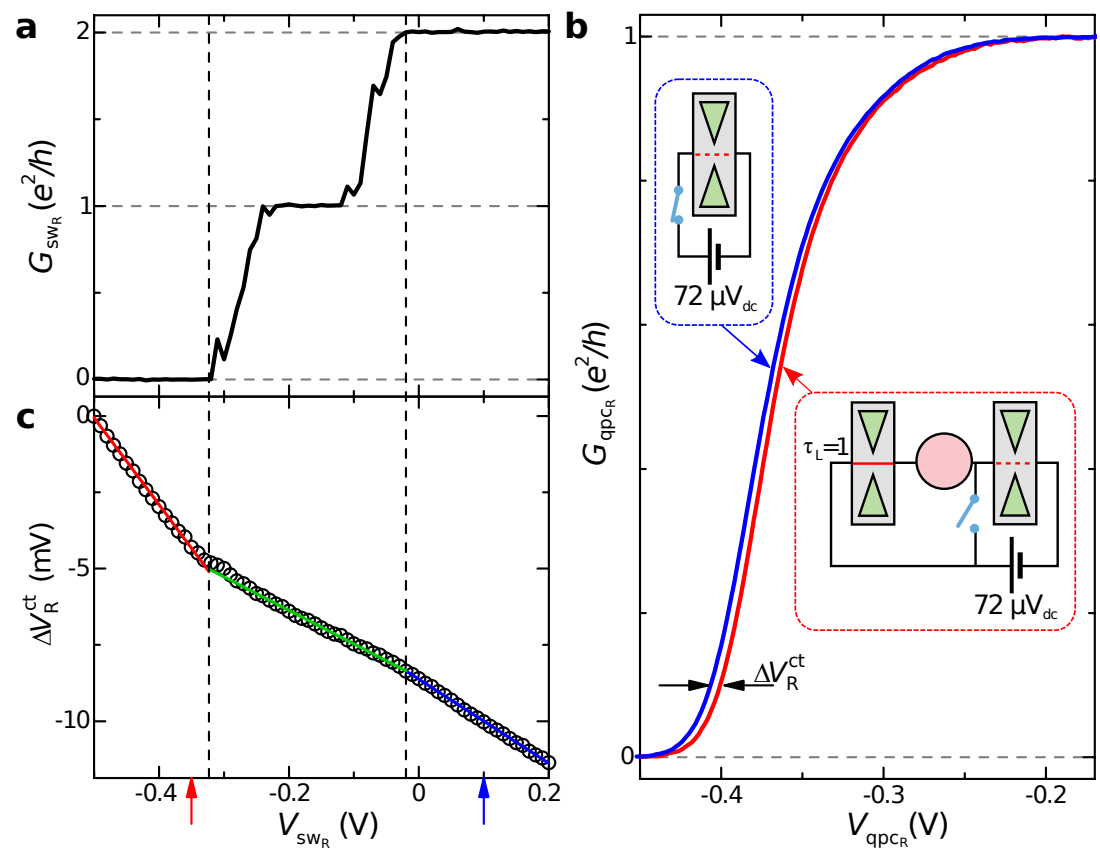

Figure B.7: Crosstalk characterization. a, Intrinsic conductance $G_{\mathrm{sw}_{R}}$ across the right switch gate versus the voltage applied on this gate $V_{\mathrm{sw}_{R}}$. The red and blue arrows on the $x$-axis indicates the voltages that define respectively the 'open' and 'closed' positions of the switch, see the inset of $\mathbf{b}$ (a zero-conductance in red is an open circuit). b, Intrinsic conductance $G_{\mathrm{qpc}_{R}}$ across the $\mathrm{QPC}_{R}$ versus its voltage gate $V_{\mathrm{qpc}_{R}}$. The red and blue traces correspond respectively to the 'open' and 'closed' positions of the right switch. The capacitive influence is measured by the spacing $\Delta V_{R}^{\text {ct }}$ between the two traces (focussing on small conductances, see text). c, The spacing $\Delta V_{R}^{\text {ct }}$ between a reference trace of $G_{\mathrm{qpc}_{R}}$ measured for a $V_{\mathrm{sw}_{R}}=-0.5 \mathrm{~V}$ and another one is displayed versus $V_{\mathrm{sw}_{R}}$. The vertical dashed lines delimit the $G_{\mathrm{sw}_{R}}=0$ and $2 G_{K}$ quantum Hall plateaus.

minimize the difference between the voltage bias of $\mathrm{QPC}_{R}$ in both configurations.

We have measured the dependence of this spacing for other positions of the switch than the two we have chosen for its 'open' and 'closed' configurations $\left(V_{\mathrm{sw}_{R}}=-0.35 \mathrm{~V}\right.$ and $0.1 \mathrm{~V}$ respectively). We see in Fig. B.7k that this dependence is relatively simple (as we might expect for an electrostatic effect in this geometry); it is piecewise linear.

\section{B.2 Ohmic contact}

The presence of the central ohmic contact (in purple in Fig. B.1) and its good quality are essential for the experiments reported in this thesis. It has two important 
features: (i) the coherence of electron is lost when they cross it because the energy level spacing in this metal is completely negligible and (ii) its connection to the $2 \mathrm{DEG}$ is perfect at our accuracy.

\section{B.2.1 Mean energy level spacing in the island}

The energy level spacing depends on the dimension of the conductor. It is given by the following formulas (first equations in [65]):

$$
\begin{aligned}
\delta E_{2 D} & =(1 / \pi) \frac{\hbar^{2} \pi^{2}}{m L^{2}} \\
\delta E_{3 D} & =1 /\left(3 \pi^{2} N_{\mathrm{at}}\right)^{1 / 3} \frac{\hbar^{2} \pi^{2}}{m L^{2}}
\end{aligned}
$$

where $N_{\text {at }}$ is the number of atoms, $m=m_{e}=9.1 \times 10^{-31} \mathrm{~kg}$ is the mass of the electrons and $L \sim 1.5 \mu \mathrm{m}$ is the typical length of the material. We estimate $N_{\text {at }} \sim$ $(L / a)^{3}$ with $a \sim 0.1$ or $1 \mathrm{~nm}$ as a typical interatomic distance. These numbers give $\delta E_{3 D} / k_{B} \approx 0.1$ or $1 \mu \mathrm{K}$. This is far below our lowest base temperature of $6 \mathrm{mK}$. At this temperature, too many discrete energy levels will be activated to still consider the density of state as discrete.

The presence of this metallic ohmic contact is crucial, because $\delta E_{2 D} \approx 18 \mathrm{mK}$ (here we should consider the effective mass $m^{*}=0.067 m_{e}$ of the electrons in GaAs). This level spacing (for the 2DEG without ohmic contact) would be larger than our base temperature.

We distinguish our metallic island (that has a continuous density of state) from the quantum dots (that have a discrete one) used in other similar experiments as [66, 8]. Having discrete levels would have dramatically changed the physics involved in our device where coherent transport through the island connected by few electronic channels is fully negligible.

\section{B.2.2 Connection of the micron-sized ohmic contact to the 2DEG}

At our experimental accuracy, we have not measured any reflection at the interface between the ohmic contact and the 2DEG for the first electronic channel transmitted through each QPC. The measurement protocol is explained here, it is similar to the way we calibrate the gain of the measurement lines in Appendix E.1.2. However, this measurement is independent of any gain calibration. Measurement artifacts could only come from offsets (which are smaller than experimental noise for this characterization). 
We measure the signal reflected back $V_{i, i}$ on each QPC when all the QPCs are at $\tau=0$ and then when they all are at $\tau=1$. The ratio of the two measurements will be linked to the transmissions by:

$$
v\left(1-\frac{V_{i, i}(\tau=1)}{V_{i, i}(\tau=0)}\right)=\left(\frac{1}{\tau_{i}}+\frac{1}{\tau_{j}+\tau_{k}}\right)^{-1}
$$

where $v$ is the filling factor of the IQHE. Let us define the normalized signals $v_{i, i} \triangleq v\left(1-\frac{V_{i, i}(\tau=1)}{V_{i, i}(\tau=0)}\right)$. We have 3 equations and 3 unknowns, solving the system of equations yields the expression of $\tau_{i}$ :

$$
\tau_{i}=\frac{v_{i, i}^{2}+\left(v_{j, j}-v_{k, k}\right)^{2}-2 v_{i, i}\left(v_{j, j}+v_{k, k}\right)}{2\left(v_{i, i}-v_{j, j}-v_{k, k}\right)}
$$

The measurements and the deduced transmissions are given in Table B.2.

\begin{tabular}{|c|c|c||c|}
\hline $\mathrm{QPC}_{i}$ & $V_{i, i}(\tau=0)(\mu \mathrm{V})$ & $V_{i, i}(\tau=1)(\mu \mathrm{V})$ & $\tau_{i}$ \\
\hline \hline $\mathrm{QPC}_{1}$ & $3.82606 \pm 0.00002$ & $2.97598 \pm 0.00002$ & $0.99967 \pm 0.00006$ \\
\hline $\mathrm{QPC}_{2}$ & $3.79625 \pm 0.00002$ & $2.95269 \pm 0.00002$ & $0.99991 \pm 0.00006$ \\
\hline $\mathrm{QPC}_{3}$ & $3.80858 \pm 0.00002$ & $2.96212 \pm 0.00002$ & $1.00031 \pm 0.00006$ \\
\hline
\end{tabular}

Table B.2: Transmission of the outermost channel to the micron-sized central ohmic contact. These data are taken at the filling factor $v=3$. Each configuration ( $\tau=0$ or $\tau=1$ ) have been acquired during $\sim 20$ minutes. The errors bars are standard error on the mean value.

Let us recall that this measurement is free of any gain calibration as we divide two measurements made with the same amplification line. The offsets in the voltage measurement are typically of $-7 \times 10^{-11} \mathrm{~V}$. Such an offset will change the transmission measured of $\Delta \tau=2 \times 10^{-5}$, which is smaller than the measurement error bars. That is why we can claim that the connection between the micron-sized ohmic contact and the 2DEG is perfect within our accuracy.

This connection is weaker for inner channels. It remains good at $10^{-3}$ for the second channel at $v=3$. The last channel is not transmitted to the central ohmic contact on the sides of $\mathrm{QPC}_{1}$ and $\mathrm{QPC}_{3}$, the transmission remains at 2 while it reaches $\tau_{2}=3.00221$ for $\mathrm{QPC}_{2}$. Despite the imperfection of the connection of the ohmic contact to the 2DEG for these inner channels (probably because of its small size), we are satisfied since we are not using these inner channels in our experiments. 


\section{Appendix C}

\section{Single electron transistor}

This appendix is a pedestrian derivation of the conductance of the SET based on the perturbative approach for the tunneling through the junctions explained in [91, 53]. This theory (usually called the 'orthodox theory') is well established. Here we aim to present the origin of quantities we will use all along this thesis (the charging energy $E_{C}$, the conductance of the device, etc.). Note that this theory is semi-classical (the only 'quantum' ingredient is the amplitude of the tunneling probability).

\section{C.1 Charging Energy}

Let us consider the electrical circuit sketched in Fig. C.1. The central node is surrounded by capacitors. The border of the so-called 'island' is delimited by a dashed pink line. The total charge $Q$ in the island is an integer multiple of the elementary charge $Q=N e$.

The electrostatic energy of the island is given by the sum of the energy stored in the capacitors $C_{1}, C_{2}, C_{g}$ and the potential electrostatic energy given by the voltage sources $V_{1}, V_{2}, V_{g}$ to bring by the charges $q_{1}, q_{2}, q_{g}$ onto the island:

$$
E_{e l}=\frac{1}{2}\left(\frac{q_{1}^{2}}{C_{1}}+\frac{q_{2}^{2}}{C_{2}}+\frac{q_{g}^{2}}{C_{g}}\right)+q_{1} V_{1}+q_{2} V_{2}+q_{g} V_{g}
$$

We can simplify this expression using Kirchoff laws and the definition of the charge state $N \triangleq\left(q_{1}+q_{2}+q_{g}\right) / e$ :

$$
\left\{\begin{aligned}
U_{1}-U_{g} & =V_{g}-V_{1} \\
U_{2}-U_{g} & =V_{g}-V_{2} \\
q_{1}+q_{2}+q_{g} & =e N
\end{aligned}\right.
$$




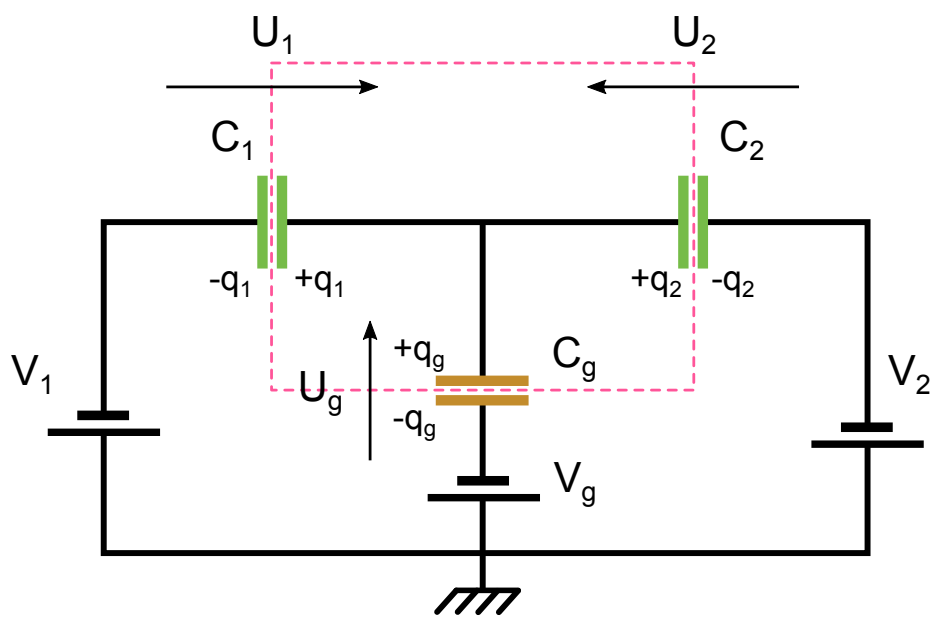

Figure C.1: Single electron transistor (SET) circuit. Each branch surrounding the central node is labeled with an index $i=1,2, g$. $U_{i}$ indicates the voltage drop across the capacitor $i$, and $\pm q_{i}$ is the charge accumulated on each side of the capacitor $i$.

where $U_{i}=q_{i} / C_{i}$ is the voltage drop across the capacitor $i=\{1,2, g\}$. If we substitute the solution of this system of equation in the Eq. (C.1), we get

$$
\begin{aligned}
E_{e l}= & \frac{1}{2\left(C_{1}+C_{2}+C_{g}\right)}\left[e^{2} N^{2}+\right. \\
& -C_{1}\left(C_{2}\left(V_{1}-V_{2}\right)^{2}+C_{g}\left(V_{1}-V_{g}\right)^{2}\right)-C_{2} C_{g}\left(V_{2}-V_{g}\right)^{2} \\
& \left.+2 e N\left(C_{1} V_{1}+C_{2} V_{2}+C_{g} V_{g}\right)\right]
\end{aligned}
$$

We are interested in knowing which $N$ will minimize the electrostatic energy $E_{e l}$ for a given configuration of voltages $V_{i}$. This is why we can add or remove terms that does not depend on $N$. Let us define

$$
q \triangleq-\left(C_{1} V_{1}+C_{2} V_{2}+C_{g} V_{g}\right)
$$

By removing the terms independent of $N$, and adding $q^{2} /\left(2\left(C_{1}+C_{2}+C_{g}\right)\right)$, it comes:

$$
E_{e l}=E_{C}(N-q / e)^{2}
$$

where we have used the charging energy $E_{C}$ defined by: $E_{C} \triangleq e^{2} / 2 C_{\Sigma}$, with $C_{\Sigma} \triangleq C_{1}+C_{2}+C_{g}$.

Unlike $q_{1}, q_{2}$ or $q_{g}$, the variable $q$ has the unit of a charge, but it is continuous. The electrostatic energy only depends on this variable, it is then easy to minimize: when $-1 / 2 \leqslant q / e \leqslant 1 / 2, N=0$, when $1 / 2 \leqslant q / e \leqslant 3 / 2, N=1$, and so on. 
The minimal electrostatic energy is $e$-periodic, and at each half-integer values of $q / e$ there are two adjacent values of $N$ that minimize $E_{e l}$. It means one can tune $q$ to put the system in a degenerate state. Dealing with this degenerate state would be central in this thesis.

\section{C.2 Coulomb diamonds}

So far we have studied a static situation. Let us now define the processes that could change the number of electrons $N$ on the island. We distinguish between the capacitors $C_{1}$ and $C_{2}$ that could leak electrons by a tunnel process (that will be discussed in the Appendix C.3 and the capacitor $C_{g}$ that will remain a perfect capacitor.

There are four possible processes: an increment of $N$ by a charge (i) coming from the reservoir of the voltage source $V_{1}$ or (ii) coming from $V_{2}$, or a decrement of $N$ by a charge (iii) going to the voltage source $V_{1}$ or (iv) going to the voltage source $V_{2}$. The electrostatic energy before and after these process is not the same.

For instance, let us consider the process (i). Replacing $q_{1} \longmapsto\left(q_{1}+1\right)$ and $N \longmapsto(N+1)$ in the same calculations as in the previous section leads to $E_{e l}^{\text {after }}=$ $E_{C}((N+1)-q / e)^{2}$. Here we also have to take into account the work done by the voltage source $V_{1}$ to bring a new charge $e$ onto the island. For this process (i), the difference of energy after (i) and before (i) is given by $\Delta E_{1}^{+}=E_{e l}(N+$ 1) $-E_{e l}(N)+e V_{1}$, where the superscript ' + ' symbolize the incrementation of the number of electrons on the island and the subscript ' 1 ' indicates the voltage source that moves the electron. this in:

In general, $\Delta E_{i}^{\eta}=E_{e l}(N+\eta)-E_{e l}(N)+\eta e V_{i}$ with $\eta= \pm 1$, we can simplify

$$
\Delta E_{i}^{\eta}=2 E_{C}(\eta N+1 / 2-\eta q / e)+\eta e V_{i}
$$

Let us consider the case where $N=0$, with an anti-symmetric biasing $V / 2 \triangleq$ $V_{1}=-V_{2} \triangleq \varepsilon_{i} V_{i}$. And let us also define $N_{g} \triangleq C_{g} V_{g} / e$, so that $q=-\left(\left(C_{1}-\right.\right.$ $\left.C_{2}\right) V / 2+e N_{g}$ ). The processes (i-iv) can occur only if $\Delta E_{i}^{\eta}>0$ :

$$
2 E_{C}\left[1 / 2+\eta\left(\frac{\left(C_{1}-C_{2}\right) V}{2 e}+N_{g}\right)\right]+\eta \varepsilon_{i} e V / 2>0
$$

As one can see in Fig. C.2 that the region of the $\left(N_{g}, e V\right)$ plane where the four processes (i-iv) are forbidden is an horizontally stretched diamond delimited 1 by the following equations:

\footnotetext{
${ }^{1}$ One can neglect the term $2 E_{C} \frac{C_{1}-C_{2}}{e^{2}}=\frac{C_{1}-C_{2}}{C_{\Sigma}}$, and the sign of the inequalities are given by the sign of $\eta \varepsilon_{i}$.
} 


$$
e V=\frac{4 \eta E_{C}\left(1 / 2+2 \eta N_{g}\right)}{2 E_{C} \frac{C_{1}-C_{2}}{e^{2}}+\varepsilon_{i}}
$$

If $C_{1}=C_{2}$, the diamond is symmetric for $N_{g}$ positive or negative. Otherwise, the slopes of the diamond give access to the asymmetry between the capacitances $C_{1}$ and $C_{2}$. But whatever the asymmetry, the total height of the diamond is given by $4 E_{C}$.

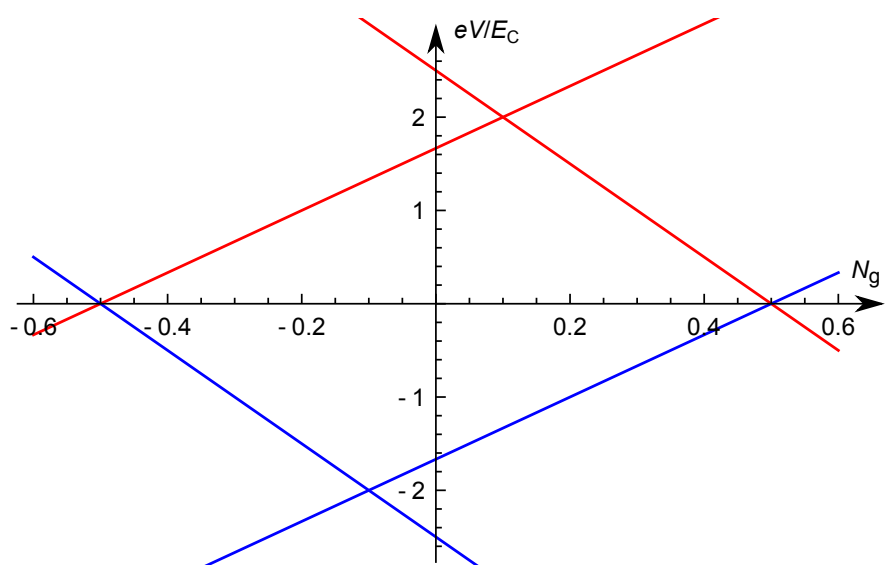

Figure C.2: Coulomb diamond. The four lines of the diamond are defined by Eq. (C.8). No tunneling process can occur above (below) the red (blue) lines. The conductance of the SET is zero inside the diamond. The total height of the diamond is $4 E_{C}$. In this plot: $e=1, C_{1}=1.0$ and $C_{2}=0.9$.

Experimentally, the fact that all the processes are forbidden means that the charge state $N$ of the island is frozen. No current can flow through it, this phenomenon is called '(static) Coulomb blockade'. This diamond pattern repeats itself at each integer value of $N_{g}$, just as $E_{e l}$ is $e$-periodic. On the $y$-axis, we see that when $e V \gtrsim E_{C}$, one leaves the diamond, the blockade regime.

In practice, the experiment will be run at finite temperature. This will smear the diamonds and allow a current to flow in the vicinity of the lines defined by Eq. (C.8). To be more quantitative, we should introduce the master equation that rules the statistic thermal population of each charge state.

\section{C.3 Master equation}

The processes that change the charge state of the island are due to tunneling of electrons. These events happen randomly when the energy is available ${ }^{2}$, Given

\footnotetext{
${ }^{2}$ We do not consider co-tunneling: the tunneling of an electron to a virtual state (which is higher in energy) and then, from this virtual state to a real state.
} 
$N_{\text {states }}$ possible charge states, we want to know which charge state is populated in given operating conditions.

The evolution of the probability $p_{N}(t)$ for the island to have $N$ electrons at time $t$ is ruled by the following master equation:

$$
\begin{aligned}
\frac{\mathrm{d}}{\mathrm{d} t} p_{N}(t)= & -\left(\Gamma^{+}(N)+\Gamma^{-}(N)\right) p_{N}(t) \\
& +\Gamma^{-}(N+1) p_{N+1}(t)+\Gamma^{+}(N-1) p_{N-1}(t)
\end{aligned}
$$

where the $\Gamma$ are tunneling rates. These tunneling rates depend on the working conditions, for instance, if the island is in charge state $N$ and it is easy to go to charge state $N+1$, the rate $\Gamma^{+}(N)$ will be high. $1 / \Gamma$ is the typical time for a process to occur.

In the Eq. (C.9), the two first terms with sign '-' stands for the processes that depopulates the charge state $N$, while the two last terms stands for the processes that populates this state. Each rate $\Gamma$ of Eq. (C.9) contains both the contributions for tunneling process from/to the reservoir of the voltage sources $V_{1}$ and $V_{2}$ : $\Gamma^{\eta}=\Gamma_{1}^{\eta}+\Gamma_{2}^{\eta}$.

The current leaking through the capacitor $C_{1}$, or in more accurate words, the current flowing through the tunnel junction \#1 is $e$ times the net number of charges that go through the junction per unit of time:

$$
I_{1}=e \sum_{N_{\text {states }}}\left(\Gamma_{1}^{+}(N)-\Gamma_{1}^{-}(N)\right) p_{N}
$$

We assume the stationary hypothesis: $\frac{\mathrm{d}}{\mathrm{d} t} p_{N}(t)=0$. In this case, there is no charge temporary stored in the island, and the current $I \triangleq I_{1}=I_{2}$ through the two junctions is the same. This hypothesis is valid since the tunneling rates $\Gamma$ are very fast compared to any change in the working conditions. Indeed, the tunneling rates are given by:

$$
\Gamma=\frac{G_{\infty}}{e^{2}} \frac{\Delta E}{\exp \left(\Delta E / k_{B} T\right)-1}
$$

where $G_{\infty}=\tau e^{2} / h$ and $\tau$ is the intrinsic transmission of the junction. At small bias $V$, and close to the charge degeneracy $N_{g} \sim 1 / 2, \Gamma \sim \tau k_{B} T / h$. With a typical value of $\tau=0.10$ and a temperature of $T=6 \mathrm{mK}, 1 / \Gamma \sim 10^{-7} \mathrm{sec}$. This time is much smaller than the frequencies we use in our measurement which are in the $\mathrm{kHz}$ range or even lower. 


\section{C.4 Coulomb blockade oscillations}

Now we have all the ingredients to trace the electrical conductance of the device as a function of $N_{g}$ (which is proportional to the gate voltage $V_{g}, N_{g} \triangleq C_{g} V_{g} / e$ ). The conductance will show $e$-periodic oscillations with maxima placed at the charge degeneracy points.

Let us consider only two charge states $N=0$ or 1 . One can solve the master equation Eq. (C.9) and use $p_{0}+p_{1}=1$ to get $p_{0}$ and $p_{1}$. Using Eq. (C.10), it comes:

$$
I / e=\frac{\Gamma_{1}^{+}(0) \Gamma_{2}^{-}(1)-\Gamma_{1}^{-}(1) \Gamma_{2}^{+}(0)}{\Gamma^{+}(0)+\Gamma^{-}(1)}
$$

And using Eqs. (C.6) and (C.11), we get the explicit expression of $I\left(G_{1 \infty}, G_{2 \infty}\right.$, $\left.T, V_{1}, V_{2}, q\left(V_{1}, V_{2}, V_{g}\right)\right)$. If we assume a symmetric small biasing $V / 2 \triangleq V_{1}=-V_{2}$, the first term in the series development of the conductance $G=I / V$ is:

$$
G=\frac{G_{\infty}}{2} \frac{\Delta E /\left(k_{B} T\right)}{\sinh \Delta E /\left(k_{B} T\right)}
$$

where $G_{\infty} \triangleq \frac{G_{1 \infty} G_{2 \infty}}{G_{1 \infty}+G_{2 \infty}}$ is the intrinsic serial conductance and $\Delta E \triangleq$ $2 E_{C}\left(1 / 2-N_{g}\right)$ is related to the gate voltage $V_{g}$ (and which will be called the 'splitting in energy' in Chapter 4 , because it favors either the $N=0$ or the $N=1$ state, while at $w=0$ these two states are degenerate in energy).

Actually, we should consider more charge states to obtain $e$-periodic peaks. Figure C.3 shows the normalized conductance $G / G_{\infty}$ as a function of $q / e \propto N_{g}$ for a small biasing and for two temperatures. In this figure, we see that at higher temperatures, one has to consider many charge states in the calculations, because the thermal distribution populates several charge states. At very high temperatures, the oscillations vanishes: the charging effects are no longer visible, as the electrons are too energetic to be Coulomb blocked.

These Coulomb oscillations are a clear signature of the quantization of the charge on the island. Indeed, the $e$-periodic dependence of the conductance of the device on the gate voltage $V_{g}$ shows a precise control of the number of electrons localized on the island. 


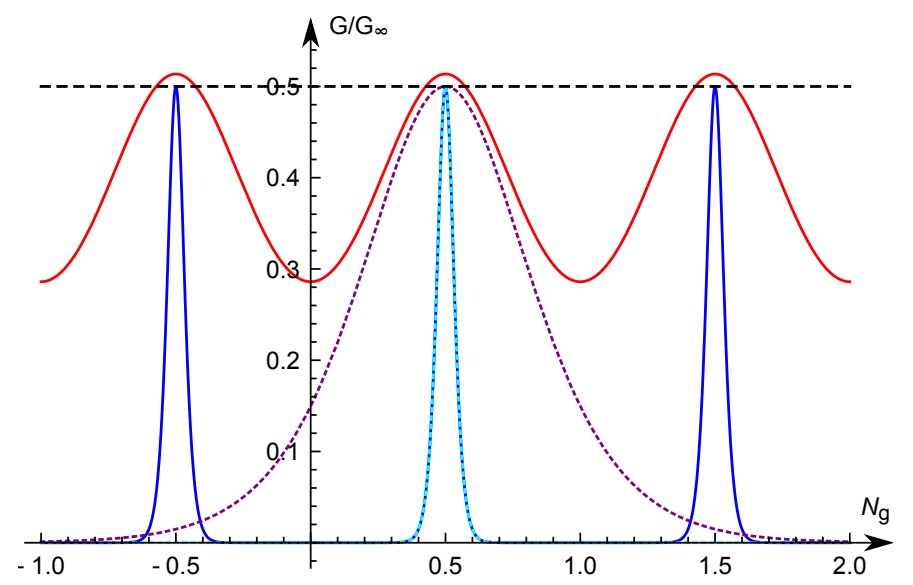

Figure C.3: Coulomb blockade oscillations of conductance. The conductance of the SET as a function of the plunger gate $N_{g}$ is plotted for two models (solid and dotted lines) and for two temperatures (blue-cyan at $10 \mathrm{mK}$ and red-violet at $100 \mathrm{mK}$ ) with $E_{C}=300 \mathrm{mK}$. The dotted lines are plotted taking into account only two charge states, while the solid ones take $N_{\text {states }}=7$. The solid lines model is not given by an accumulation of independent dotted lines. However, the higher the temperature, the higher the conductance. 
Appendix D

Primary thermometry triad at $6 \mathrm{mK}$ 


\section{ARTICLE}

\section{Primary thermometry triad at $6 \mathrm{mK}$ in mesoscopic circuits}

Z. Iftikhar ${ }^{1}$, A. Anthore', S. Jezouin ${ }^{1}$, F.D. Parmentier ${ }^{1}$, Y. Jin ${ }^{1}$, A. Cavanna ${ }^{1}$, A. Ouerghi', U. Gennser ${ }^{1}$ \& F. Pierre ${ }^{1}$

Quantum physics emerge and develop as temperature is reduced. Although mesoscopic electrical circuits constitute an outstanding platform to explore quantum behaviour, the challenge in cooling the electrons impedes their potential. The strong coupling of such micrometre-scale devices with the measurement lines, combined with the weak coupling to the substrate, makes them extremely difficult to thermalize below $10 \mathrm{mK}$ and imposes in situ thermometers. Here we demonstrate electronic quantum transport at $6 \mathrm{mK}$ in micrometre-scale mesoscopic circuits. The thermometry methods are established by the comparison of three in situ primary thermometers, each involving a different underlying physics. The employed combination of quantum shot noise, quantum back action of a resistive circuit and conductance oscillations of a single-electron transistor covers a remarkably broad spectrum of mesoscopic phenomena. The experiment, performed in vacuum using a standard cryogen-free dilution refrigerator, paves the way towards the sub-millikelvin range with additional thermalization and refrigeration techniques.

\footnotetext{
${ }^{1}$ Centre de Nanosciences et de Nanotechnologies, CNRS, Univ Paris Sud-Université Paris-Saclay, Université Paris Diderot-Sorbonne Paris Cité,
} 91120 Palaiseau, France. Correspondence and requests for materials should be addressed to F.P. (email: frederic.pierre@u-psud.fr). 
A dvances towards lower temperatures are instrumental in the fundamental exploration of quantum phenomena. In the context of quantum electronics, typical examples are the exploration of the correlated fractional quantum Hall physics $^{1-5}$, of the quantum criticality, for example, with multichannel Kondo nanostructures ${ }^{6-8}$, or of the quantum aspects of heat ${ }^{9-12}$. Although commercial dilution refrigerators readily achieve temperatures in the $5-10 \mathrm{mK}$ range at the mixing chamber, the pertinent value is the temperature of the electrons within the cooled quantum circuits. Owing to microwave heating, insufficient thermal contacts and electrical noise transmitted through the measurement lines, this electronic temperature is usually well above the refrigerator base temperature. Consequently, only rare examples demonstrate electronic temperatures significantly below $10 \mathrm{mK}$ in quantum circuits. Moreover, the concept of temperature pervades the laws of physics, and its accurate knowledge is generally imperative whenever comparing experimental measurements with theoretical predictions; however, establishing the validity of the thermometry is particularly challenging already below $50 \mathrm{mK}$. Because of the thermal decoupling between electrons and substrate, it requires a comparison of the electronic temperature determined in situ, in the same device, by different methods.

The lowest electronic temperatures in solid-state quantum circuits were obtained in large, millimetre-scale, devices that are thereby weakly sensitive to heating through the measurement lines. The lowest reported value of $3.7 \mathrm{mK}$, to our knowledge, was obtained in a large array of 600 metallic islands, each $\sim 100 \mu \mathrm{m}$ wide and interconnected by tunnel junctions ${ }^{13}$. Comparably low temperatures, of possibly $\sim 4 \mathrm{mK}$, were inferred in twodimensional (2D) electron gas (2DEG) chips in the quantum Hall regime by two different teams $s^{2,14,15}$. For the more broadly pertinent micrometre-scale mesoscopic circuits, the reported electronic temperatures are significantly higher. We note the remarkably low value of $9 \mathrm{mK}$ determined with current fluctuation measurements across a quantum point contact (QPC) in a $2 \mathrm{DEG}^{16}$. Although single-electron devices are particularly challenging, because of their high charge sensitivity, comparably low electronic temperatures, down to $\sim 10 \mathrm{mK}$, were recently demonstrated in 2 DEG quantum dots $6,8,17$.

Here we investigate three primary electronic thermometers, and demonstrate quantum electronic transport at $6 \mathrm{mK}$ in micrometre-scale mesoscopic circuits. For this purpose, the experiment is performed on a highly tunable 2DEG nanostructure, that can be set by field effect to different circuit configurations. The complementary underlying physics of the thermometry methods give us access to different facets of the electronic temperature, and cover a broad spectrum of mesoscopic quantum phenomena. Whereas quantum shot noise thermometry measures the temperature of the electronic Fermi quasiparticles, through their energy distribution ${ }^{18}$, the quantum back action of a resistive circuit also probes the temperature of the electromagnetic environment ${ }^{19}$. In contrast, the temperature inferred from the conductance oscillations of a single-electron transistor (SET) is very sensitive to charge fluctuations induced by non-thermal high-energy photons ${ }^{20}$. At the applied magnetic field $B=1.4 \mathrm{~T}$, we find with the quantum shot noise measured across a voltage-biased QPC $T_{\mathrm{N}} \simeq 6.0 \pm 0.1 \mathrm{mK}$. From the conductance peaks across the device set to a SET configuration, we obtain $T_{\mathrm{CB}} \simeq 6.3 \pm 0.3 \mathrm{mK}$. From the dynamical Coulomb blockade conductance dip across two separate realizations of a QPC in series with a resistance, we find $T_{\mathrm{DCBL}} \simeq 6 \pm 1 \mathrm{mK}$ and $T_{\mathrm{DCBR}} \simeq 6.5 \pm 1 \mathrm{mK}$. The observed agreement between the three primary thermometers establishes their validity on an extended temperature range.

\section{Results}

Cooled tunable mesoscopic circuit. A colourized electron micrograph of the measured device is shown in Fig. 1a, with the corresponding circuit schematic displayed Fig. 1b. A high-mobility 2DEG is located $105 \mathrm{~nm}$ below the surface of a $\mathrm{Ga}(\mathrm{Al}) \mathrm{As}$ heterojunction. It is confined by etching within the darker grey areas delimited by bright lines, and can be tuned in situ, by field effect, with the bias voltages applied to metallic gates deposited at the surface and capacitively coupled to the 2DEG (colourized green, yellow and blue in Fig. 1a). The metallic split gates at the top-left $\left(\mathrm{QPC}_{\mathrm{L}}\right)$ and bottom-right $\left(\mathrm{QPC}_{\mathrm{R}}\right)$ of Fig. 1a (colourized green) are used to form QPCs in the 2DEG. Note that the split gates at the top-right of Fig. 1a (colourized yellow) is here set to fully deplete the 2DEG underneath, thereby closing the gate, and can be ignored. The buried 2DEG is galvanically connected, with a negligible interface resistance, to the central micrometre-sized metallic island (colourized red). For this purpose, the metallic island was diffused into the $\mathrm{Ga}(\mathrm{Al}) \mathrm{As}$ heterojunction by thermal annealing. The lateral continuous gates at the surface (colourized blue) implement the equivalent of short-circuit switches in parallel with the island (blue switches in Fig. 1b). The experiments are performed with a magnetic field
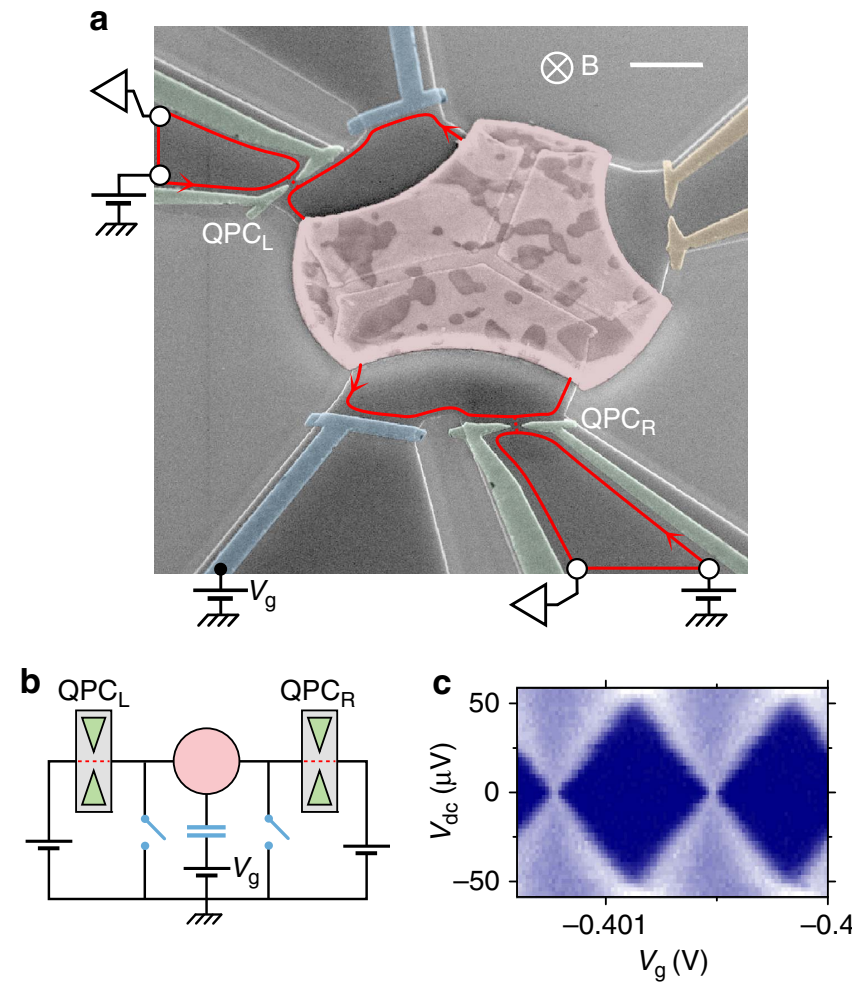

Figure 1 | Cooled electrical nanostructure. (a) Coloured micrograph of the measured device. Top-right scale bar length, $1 \mu \mathrm{m}$. The micrometre-scale metallic island (red) is connected to $200 \mu \mathrm{m}$ wide electrodes (represented as white circles) through two QPCs (green split gates) formed in a buried 2DEG(darker grey). The lateral gates (blue) implement the switches shown in $\mathbf{b}$ by field effect. The sample is immersed in a magnetic field $B$ corresponding to the integer quantum Hall regime, with the current propagating along the edge (red lines) in the direction indicated by arrows. (b) Schematic electrical circuit. Using the switches, the same device can be tuned in situ into a voltage-biased QPC, a SET or a QPC embedded into a resistive circuit. (c) Charging energy characterization, for Coulomb blockade phenomena. With the device tuned into a SET, $E_{C}=25 \pm 1 \mu \mathrm{eV}$ is obtained from the height of the diamond patterns in the SET conductance (larger values shown brighter) measured versus gate $\left(V_{g}\right)$ and bias $\left(V_{\mathrm{dc}}\right)$ voltages. 
$B$ applied perpendicular to the $2 \mathrm{DEG}$, which corresponds to the quantum Hall regime at integer filling factors $v=6,3$ and 2 for $B=1.4, \quad 2.7$ and $3.8 \mathrm{~T}$, respectively. In this regime the current flows along $v$ chiral edge channels, represented as a single red line with the propagation direction indicated by arrows in Fig. 1a. Note that the quantum Hall effect is not necessary for the investigated primary thermometers (although it allows eliminating possible heating artefacts in the quantum shot noise thermometry, see Discussion). An important device parameter is the single-electron charging energy $E_{C} \equiv e^{2} / 2 C$ of the central metallic island, with $C$ its overall geometrical capacitance and $e$ the elementary electron charge. In particular, $E_{C}$ sets the temperature scale extracted from Coulomb blockade thermometry. The charging energy is most straightforwardly determined by setting the device in the SET configuration, with the shortcircuit switches open (as shown in Fig. 1a,b) and $\mathrm{QPC}_{\mathrm{L}, \mathrm{R}}$ tuned to tunnel contacts. The SET conductance is plotted in Fig. 1c (higher values shown brighter) versus the capacitively coupled gate voltage $V_{\mathrm{g}}$ and the applied drain-source $\mathrm{dc}$ voltage $V_{\mathrm{dc}}$. The charging energy is directly related to the periodic 'Coulomb diamond' patterns in Fig. 1c: $E_{C}=\left|e V_{\mathrm{dc}}^{\max }\right| / 2 \simeq 25 \pm 1 \mu \mathrm{eV}$, with $V_{\mathrm{dc}}^{\max }$ the diamonds' maximum dc voltage.

Electronic current fluctuations. The current across a voltage-biased quantum coherent conductor fluctuates because of the thermal agitation (the Johnson-Nyquist noise) and the granularity of charge transfers (the shot noise) ${ }^{18}$. These fluctuations give information on the charge of the carriers, for example, in the fractional quantum Hall regimes $3,16,21,22$, as well as on the statistics of the charge transfers ${ }^{23-25}$, and also provide a very robust primary thermometer for the electronic temperature ${ }^{26}$.

We have measured the current fluctuations across the device tuned into a voltage-biased QPC (schematic shown in top panel of Fig. 2a, see Supplementary Note 2 for details on the current fluctuations measurement set-up). For this purpose, the right short-circuit switch in Fig. 1b was effectively closed, by applying $V_{\mathrm{g}}=0$ to the continuous gate adjacent to $\mathrm{QPC}_{R}$. Thereby, the $2 \mathrm{DEG}$ is not depleted and the edge current flows underneath the gate without back-scattering, implementing an ideal closed switch (see Supplementary Fig. 1).

The dependence with bias voltage $V_{\mathrm{dc}}$ of the current fluctuations' spectral density, $S_{\mathrm{I}}\left(V_{\mathrm{dc}}\right)$, is directly related to the electrons' energy distribution (including in out-of-equilibrium situations $\left.s^{27,28}\right)$. For a short quantum conductor, the excess spectral density $\Delta S_{\mathrm{I}}\left(V_{\mathrm{dc}}\right) \equiv S_{\mathrm{I}}\left(V_{\mathrm{dc}}\right)-S_{\mathrm{I}}(0)$ can be calculated in the standard framework of the scattering approach ${ }^{29-31}$. It reads ${ }^{18}$ :

$$
\Delta S_{\mathrm{I}}=\frac{2 e^{2}}{h} \sum_{n} \tau_{n}\left(1-\tau_{n}\right) \times\left[e V_{\mathrm{dc}} \operatorname{coth}\left(\frac{e V_{\mathrm{dc}}}{2 k_{\mathrm{B}} T}\right)-2 k_{\mathrm{B}} T\right]
$$

where the quantum conductor is described as a set of independent conduction channels, indexed by the label $n$, each characterized by a transmission probability $\tau_{n}$, and with $k_{\mathrm{B}}(h)$ the Boltzmann (Planck) constant. Note that the noise added by the amplification chain is cancelled out by considering the excess spectral density $\Delta S_{\mathrm{I}}$. Importantly, the product between the gain of the amplification chain and $\sum \tau_{n}\left(1-\tau_{n}\right)$ is given by the temperature-independent linear slope predicted at $\left|e V_{\mathrm{dc}}\right| \gg k_{\mathrm{B}} T$. Fitting the raw spectral density data based on equation 1 therefore allows a self-calibrated determination of the electronic temperature, without requiring the knowledge of $\left\{\tau_{n}\right\}$ or of the amplification gain (see Supplementary Note 2 for further details).

The symbols in the top panel of Fig. 2 a display the excess current spectral density measured at $B=1.4 \mathrm{~T}$ versus the dc bias voltage applied across the QPC, which is tuned into the advantageous configuration of a single half-transmitted conduction channel $(\tau \simeq 0.55)$. Note that in order to display the current fluctuations data in $\mathrm{A}^{2} / \mathrm{Hz}$, and, although it is not necessary for extracting the electronic temperature, the effective amplification chain gain is calibrated by matching the linear bias voltage increase in the raw spectral density at large $\left|e V_{\mathrm{dc}}\right| \gg k_{\mathrm{B}} T$ with the prediction of equation 1 for the measured $\tau=0.55$. The continuous (dashed) line shows $\Delta S_{\text {I }}$ calculated using equation 1 with $\tau=0.55$ and $T=6.0 \mathrm{mK}(T=0$, with a negative vertical offset to match the $T=6.0 \mathrm{mK}$ calculation at $\left|e V_{\mathrm{dc}}\right| \gg k_{\mathrm{B}} T$ ). Experimentally, the main difficulty is to reach a sufficient resolution to accurately extract the electronic temperature. To this aim, we developed a fully homemade cryogenic noise amplification scheme, based on high electron mobility transistors grown and nanostructured in the laboratory 32,33 . Despite the unfavourable current-voltage conversion at $v=6$ because of the low quantum Hall resistance $h / 6 e^{2} \simeq 4.3 \mathrm{k} \Omega$, we resolve $\Delta S_{\text {I }}$ with an extremely high statistical precision of $\pm 9 \times 10^{-32} \mathrm{~A}^{2} \mathrm{~Hz}^{-1}$, slightly smaller than the symbols' size.

Most directly, we have determined the electronic temperature and experimental uncertainty $T_{\mathrm{N}}=6.0 \pm 0.1 \mathrm{mK}$ from the mean value (red horizontal line in bottom panel of Fig. 2a) and statistical uncertainty of an ensemble of 131 values (symbols in bottom panel of Fig. 2a) independently obtained by separately fitting successive noise measurement sweeps $\Delta S_{\mathrm{I}}\left(V_{\mathrm{dc}}\right)$. Note that the $\Delta S_{\mathrm{I}}$ data shown in the top panel of Fig. 2a was obtained by averaging these successive sweeps (each resolved with an individual noise precision of $\left.\pm 10^{-30} \mathrm{~A}^{2} \mathrm{~Hz}^{-1}\right)$.

Coulomb blockade oscillations. At low temperatures, $T \ll E_{C} / k_{\mathrm{B}}$ the charge of a mesoscopic island connected through tunnel contacts is quantized in units of the elementary electron charge $e$. This allows for the manipulation of single electrons in circuits, which has led to the field of 'single electronics' ${ }^{19}$. Setting the device in the SET configuration (see schematic in bottom panel of Fig. 2b), charge quantization results in periodic peaks of the SET conductance $G_{\text {SET }}$ when sweeping the capacitively coupled gate voltage $V_{\mathrm{g}}$. In the presence of $\mathrm{dc}$ bias voltage, the peaks develop into periodic 'Coulomb diamond' patterns as shown in Fig. 1c. The width of these conductance peaks at zero dc bias voltage constitutes a well-known primary thermometer, frequently used in the context of mesoscopic physics. For a metallic island, with a continuous density of states and connected through tunnel contact, the SET conductance reads ${ }^{34}$ :

$$
G_{\mathrm{SET}}=\frac{G_{\infty}}{2} \frac{2 E_{C}\left(\delta V_{\mathrm{g}} / \Delta\right) / k_{\mathrm{B}} T}{\sinh \left(2 E_{C}\left(\delta V_{\mathrm{g}} / \Delta\right) / k_{\mathrm{B}} T\right)},
$$

with $G_{\infty}$ the classical (high temperature) conductance of the SET, $\Delta \simeq 712 \pm 2 \mu \mathrm{V}$ the gate voltage period and $\delta V_{\mathrm{g}}$ the gate voltage difference to charge degeneracy. Note that the Coulomb blockade thermometry is possible only with tunnel contacts. In the presence of connected conduction channels with large transmission probabilities, the quantum fluctuations of the island's charge would average out Coulomb oscillations and thereby impede the Coulomb blockade thermometry (see ref. 35 for a characterization of charge quantization versus transmission probability on the same device).

The symbols in the top panel of Fig. $2 \mathrm{~b}$ represent $G_{\text {SET }}$ measured at $B=1.4 \mathrm{~T}$ versus $\delta V_{\mathrm{g}}$. The continuous line shows the SET conductance calculated using equation 2 with $T=6.3 \mathrm{mK}$ $G_{\infty}=0.088 e^{2} / h, \Delta=711 \mu \mathrm{V}$ and $E_{C}=25 \mu \mathrm{eV}$.

Similarly to quantum shot noise thermometry, we determined the electronic temperature and statistical precision $T_{\mathrm{CB}}=6.3 \pm 0.05 \mathrm{mK}$ from an ensemble of 222 values (symbols in the bottom panel of Fig. 2b) obtained by separately fitting 
a
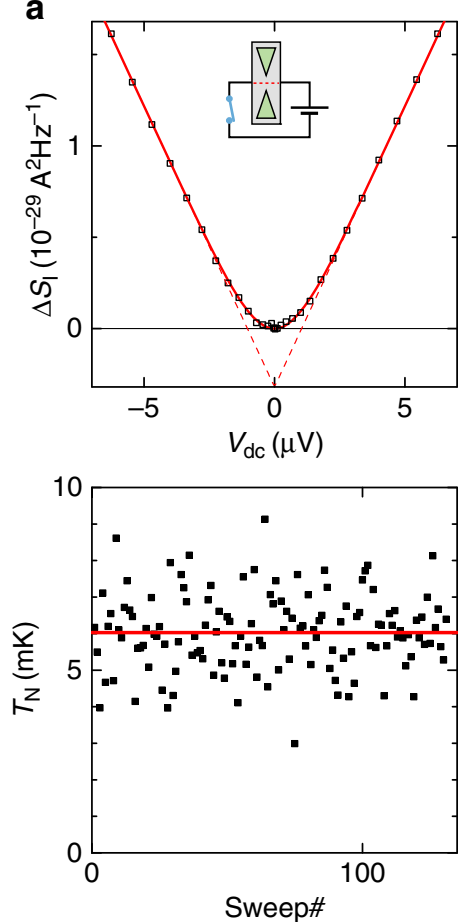

b
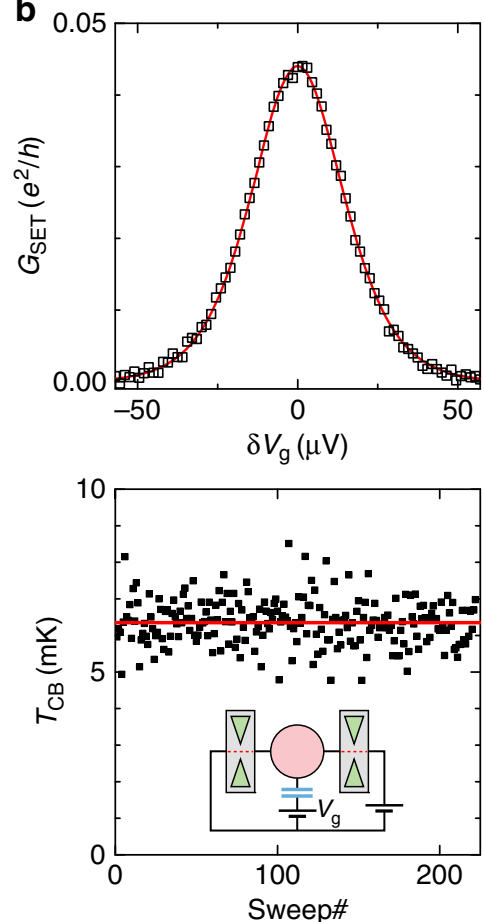

C
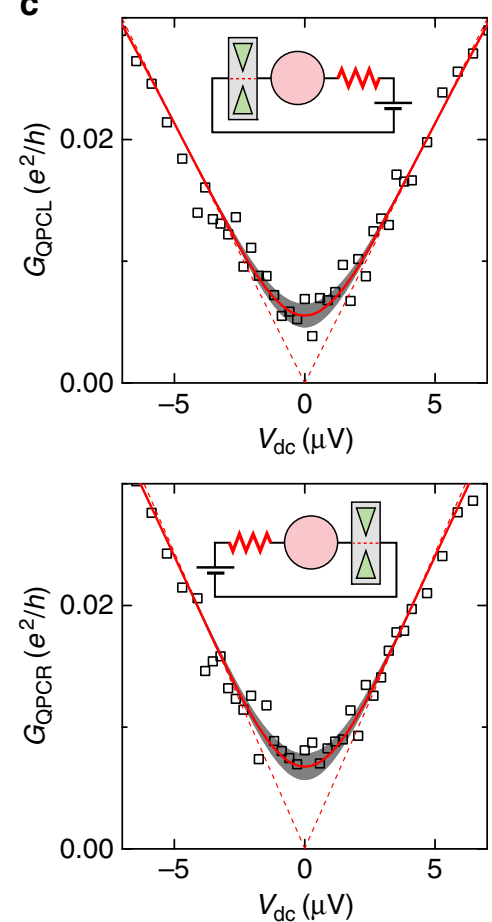

Figure 2 | Primary electronic thermometry. (a) Quantum shot noise. Symbols in the top panel represent the measured excess spectral density of the current fluctuations across a QPC biased with the dc voltage $V_{\mathrm{dc}}$ (see configuration schematic). The red continuous (dashed) line is the calculated excess current fluctuations for $T_{N}=6.0 \mathrm{mK}$ ( $T_{N}=0$, with a matching negative offset). In the bottom panel, the different electronic temperatures $T_{N}$ shown as symbols are each obtained by fitting a different (successive) voltage bias sweep of the quantum shot noise. From the statistical averaging of 131 values, we find $T_{\mathrm{N}} \simeq 6.0 \pm 0.1 \mathrm{mK}$ (horizontal red line) with an accuracy comparable to the provisional low-temperature-scale standard (PLTS-2000). (b) Coulomb blockade. Symbols in the top panel represent the measured conductance $G_{\mathrm{SET}}$ across the device tuned into a SET (see schematic in bottom panel) versus the gate voltage difference $\delta V_{\mathrm{g}}$. The continuous line is the calculated conductance for $T_{\mathrm{CB}}=6.3 \mathrm{mK}$. The different electronic temperatures $T_{\mathrm{CB}}$ represented by symbols in the bottom panel are each obtained from a different gate voltage sweep $G_{\mathrm{SET}}\left(\delta V_{\mathrm{g}}\right)$. From the averaging of 222 values, we find $T_{\mathrm{CB}} \simeq 6.3 \pm 0.05 \mathrm{mK}$ (horizontal red line). Note that the accuracy on $T_{C B}$ is limited to $\pm 0.3 \mathrm{mK}$ by our uncertainty on the charging energy, $E_{C}=25 \pm 1 \mu \mathrm{eV}$. (c) $\mathrm{Dynamical} C \mathrm{Coulomb}$ blockade. The electronic temperature $T_{\mathrm{DCB}}$ is obtained by fitting the conductance $\mathrm{G}_{\mathrm{QPCL}, \mathrm{R}}$ of $\mathrm{QPC}_{L, R}$ (symbols) versus voltage bias with the dynamical Coulomb blockade theory in the presence of a known series resistance $R=h / 2 e^{2}$ (see configuration schematic). The dashed lines display the predicted suppression of the conductance at $T=0$ and $e V_{\mathrm{dc}}<<E_{C}$, here linear in $V_{\mathrm{dc}}$. We find $T_{\mathrm{DCB}} \simeq 6 \pm 1 \mathrm{mK}(6.5 \pm 1 \mathrm{mK})$ for $\mathrm{QPC}_{\mathrm{L}}\left(\mathrm{QPC} C_{R}\right)$ from the fit shown as a continuous line in the top (bottom) panel. The estimated uncertainty of $\pm 1 \mathrm{mK}$ is displayed as a grey background.

individual sweeps of $G_{\mathrm{SET}}\left(\delta V_{\mathrm{g}}\right)$. The 222 sweeps are distributed among 14 adjacent Coulomb peaks, spreading over $10 \mathrm{mV}$ in gate voltage. We find the same electronic temperature, at experimental accuracy, for the different Coulomb peaks and also for the 15 or 16 measurements of each peak. Note that our experimental accuracy $T_{\mathrm{CB}} \simeq 6.3 \pm 0.3 \mathrm{mK}$ is limited by our resolution of the charging energy, $E_{\mathrm{C}} \simeq 25 \pm 1 \mu \mathrm{eV}$. The uncertainty is consequently much larger than the statistical precision. Note also that, despite a relatively low ac voltage of $0.35 \mu \mathrm{V}_{\text {rms }}$ applied to probe $G_{\mathrm{SET}}$, we estimate (using the master equation generalizing equation 2 to finite voltages ${ }^{19}$ ) that it is responsible for an effective increase of $0.1 \mathrm{mK}$ in $T_{\mathrm{CB}}$ (we have not corrected for this small effect). Finally, we point out that the $G_{\text {SET }}$ data shown in the top panel of Fig. $2 b$ were obtained by averaging the 222 individual sweeps.

Dynamical Coulomb blockade conductance renormalization. The conductance of a quantum coherent conductor is progressively reduced upon cooling by the quantum back action of the circuit in which it is embedded ${ }^{19}$. This phenomenon, called dynamical Coulomb blockade, results from the granularity of charge transfers combined with Coulomb interactions. It has been extensively studied, and the theory is now well established in the simplest limit of a small tunnel conductor inserted into a linear circuit (see ref. 19 and references therein; for recent developments beyond the tunnel limit see refs 36-39).

We consider here the case of a tunnel contact in series with a linear resistance $R$, as shown in the schematics of Fig. 2c. In this configuration, the conductance at zero-bias voltage (zero temperature) vanishes with temperature $T$ (bias voltage $V_{\mathrm{dc}}$ ) as $T^{2 R e^{2} / h}$ (as $\left.V_{\mathrm{dc}}^{2 R e^{2}} / h\right)$. Similarly to quantum shot noise thermometry, the equilibrium $\left(V_{\mathrm{dc}} \ll k_{\mathrm{B}} T / e\right)$ to non-equilibrium $\left(V_{\mathrm{dc}} \gg k_{\mathrm{B}} T / e\right)$ crossover provides a primary electron thermometer. In general, the electronic temperature can be extracted by fitting the conductance versus dc voltage with the full quantitative numerical prediction of the dynamical Coulomb blockade theory (see ref. 40 for a formulation involving a single numerical integration). Note that the extracted electronic temperature reflects equally the thermal energy distributions of the Fermi electron quasiparticules, and of the bosonic electromagnetic modes of the quantum circuit. The dynamical Coulomb blockade was previously used to probe the non-Fermi energy distribution of electrons driven out-of-equilibrium in the presence of a thermalized $R C$ circuit $^{41}$.

In the low-temperature and low-bias voltage regime $\left(k_{\mathrm{B}} T, e\left|V_{\mathrm{dc}}\right| \ll E_{\mathrm{C}}\right)$, the primary dynamical Coulomb blockade thermometry reduces to the simple procedure described below. The QPC conductance at low temperature $\left(T \ll E_{C} / k_{\mathrm{B}}\right)$ and at 
zero-bias voltage $V_{\mathrm{dc}}=0$ reads $^{42}$ :

$$
G_{\mathrm{QPC}}(T)=\frac{G_{\infty} \pi^{\frac{3 \mathrm{Re}^{2}}{h}}+\frac{1}{2} \Gamma\left(1+\frac{R e^{2}}{h}\right)}{2 \Gamma\left(1.5+\frac{R e^{2}}{h}\right)}\left(\frac{R e^{2}}{h} \frac{k_{\mathrm{B}} T}{E_{C}}\right)^{\frac{2 \mathrm{Re}^{2}}{h}},
$$

where $G_{\infty}$ is the tunnel conductance in the absence of dynamical Coulomb blockade renormalization and $\Gamma(x)$ is the gamma function. Extracting the temperature from the zero-bias conductance apparently requires a precise knowledge of both $G_{\infty}$ and the circuit parameters $(R, C)$. However, the necessary information is provided by the bias voltage dependence. In the non-equilibrium regime $k_{\mathrm{B}} T<<e\left|V_{\mathrm{dc}}\right|$ and at low energy compared with the single-electron charging energy $e\left|V_{\mathrm{dc}}\right|<<E_{C}$, the QPC conductance reads ${ }^{19}$ :

$$
G_{\mathrm{QPC}}\left(V_{\mathrm{dc}}\right)=\frac{G_{\infty}\left(\frac{\pi}{\gamma}\right)^{\frac{2 R e^{2}}{h}}\left(\frac{2 R e^{2}}{h}+1\right)}{\Gamma\left(2+\frac{2 R e^{2}}{h}\right)}\left(\frac{R e^{2}}{h} \frac{e\left|V_{\mathrm{dc}}\right|}{E_{C}}\right)^{\frac{2 R e^{2}}{h}},
$$

with $\gamma \simeq \exp (0.5772)$. Consequently, the bias voltage exponent gives the series resistance $R$, and one can rewrite the zero-bias voltage conductance as:

$$
G_{\mathrm{QPC}}(T)=\frac{A(R)}{B\left(G_{\infty}, R, E_{C}\right)}\left(k_{\mathrm{B}} T\right)^{\frac{2 R e^{2}}{h}}
$$

with $B\left(G_{\infty}, R, E_{C}\right) \equiv G_{\mathrm{QPC}}\left(V_{\mathrm{dc}}\right) /\left|e V_{\mathrm{dc}}\right|^{2 R e^{2} / h}$ calibrated from the conductance measured in the low-energy non-equilibrium regime, where equation 4 applies, and $A(R)$ a known function, straightforwardly obtained from equations 3 and 4 .

Here we determined the electronic temperature by setting one QPC in the tunnel regime $\left(G_{\infty} \sim 0.1 e^{2} / h\right)$, while the other QPC was tuned to fully transmit two electronic channels, thereby implementing a linear series resistance $R=h / 2 e^{2}$ (which is not renormalized by dynamical Coulomb blockade ${ }^{37-39,43}$; obtained from a very broad and flat conductance plateau owing to the quantum Hall effect $\left.{ }^{11,39}\right)$. Symbols in the top (bottom) panels of Fig. $2 c$ represent the conductance measured with the left (right) QPC in the tunnel regime, versus dc bias voltage, at $B=1.4 \mathrm{~T}$. The continuous lines display the quantitative numerical calculations of the dynamical Coulomb blockade prediction using the separately characterized $E_{C}=25 \mu \mathrm{eV}$ and $R=h / 2 e^{2} \quad$ (also corresponding to the linear bias voltage dependence), and with $G_{\infty}=0.123 e^{2} / h, T=6 \mathrm{mK}$ for the top panel $\left(G_{\infty}=0.139 e^{2} / h, T=6.5 \mathrm{mK}\right.$ for the bottom panel). The grey areas represent a temperature uncertainty of $\pm 1 \mathrm{mK}$. The dashed lines are the $T=0$ predictions of equation 4 for the same device parameters. Note that for the present circuit implementation $A\left(R=h / 2 e^{2}\right) \simeq 0.40$ and the non-equilibrium conductance increases linearly with bias voltage, as can be directly verified on the conductance data. See Supplementary Fig. 2 for a comparison between the numerically calculated dynamical Coulomb blockade predictions and the data up to larger bias voltages.

Electronic temperature versus experimental conditions Information on the limiting factors towards lower electronic temperatures $T$ in our cryogen-free dilution refrigerator is obtained by measuring $T$ for different magnetic fields $B$ and for different additional Joule powers $P_{\mathrm{J}}$ dissipated directly on the mixing chamber plate. Note that $T$ is here obtained from quantum shot noise thermometry up to $35 \mathrm{mK}$, and from the identical but faster readings of our standard $\mathrm{RuO}_{2}$ thermometer at higher temperatures. Each set of symbols in Fig. 3 corresponds to a different applied $B \in\{1.41,2.74,3.76\}$ T. For $P_{\mathrm{J}} \gtrsim 5 \mu \mathrm{W}$, we observe the usual quadratic dependence with temperature $\left(T^{2} \propto P_{\mathrm{J}}\right)$, independently of the applied $B$. However, we find that the electronic temperature at zero Joule power is higher for larger

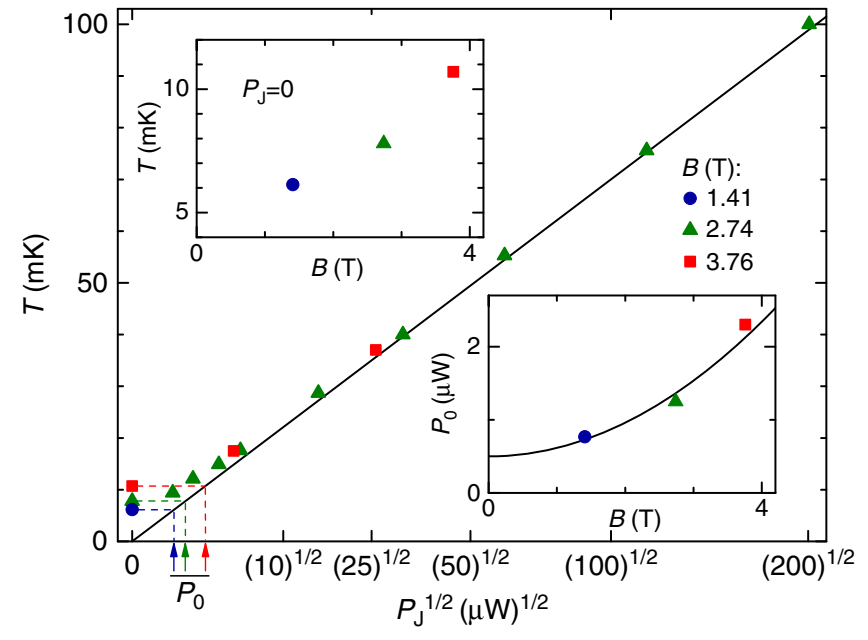

Figure 3 | Temperature versus magnetic field and Joule heating. The measured electronic temperature (symbols) is represented versus the square root of the Joule power $P_{\text {J }}$ dissipated on the mixing chamber of the dilution refrigerator, for three different values of the perpendicular magnetic field $B$. The continuous line displays $T=7 \sqrt{P_{\mathrm{J}} / 1 \mathrm{~W}} \mathrm{~K}$. Top left inset: the temperature at $P_{\mathrm{J}}=0$ (symbols) increases with the applied magnetic field $B$. Bottom right inset: the intrinsic dissipated power $P_{0}$, estimated assuming $T \propto \sqrt{P_{0}+P_{\mathrm{J}}}$ (arrows in main panel), is plotted as symbols versus magnetic field. The continuous line displays $P_{0}=0.5+0.1(B / 1 \mathrm{~T})^{2} \mu \mathrm{W}$.

magnetic fields (top left inset). Assuming that the observed relationship $T=7 \sqrt{P_{\mathrm{J}} / 1 \mathrm{~W}} \mathrm{~K}$ (straight black line in the main panel) holds at all temperatures when substituting the additional Joule power by the full dissipated power $P=P_{0}+P_{\mathrm{J}}$, we extract the refrigerator-dissipated power $P_{0}$ versus magnetic field. The corresponding $P_{0}$ values are shown as symbols in the bottom right inset. We find that the increase of $P_{0}$ with $B$ is compatible with a quadratic magnetic field dependence (continuous black line: $\left.P_{0}=0.5+0.1(B / 1 \mathrm{~T})^{2} \mu \mathrm{W}\right)$, which is a typical signature of eddy current dissipation.

\section{Discussion}

A $6 \mathrm{mK}$ electronic temperature was obtained in micrometre-scale quantum circuits using a medium-sized cryogen-free dilution refrigerator (Oxford instruments Triton, with $200 \mu \mathrm{W}$ of cooling power at $100 \mathrm{mK}$ ), with the sample in vacuum and in the presence of a $1.4 \mathrm{~T}$ magnetic field. At larger magnetic fields $B$, we observe a temperature increase that corresponds to an additional dissipated power quadratic in $B$, as typically expected for eddy currents. In our cryogen-free refrigerator, the underlying vibrations originate from the pulse tube.

The sample environment and wiring shown Fig. 4 offers a proven guideline to ultra-low electronic temperatures with an all-purpose set-up, including 35 measurement lines and a top-loaded sample holder. Although additional details are provided in the Supplementary Note 1, we here briefly point out several key ingredients. The sample is strongly protected from spurious high-energy photons, by two shields at base temperature. The most important thermal anchoring of the measurement lines at base temperature is performed by dipping insulated copper wires into silver epoxy very close to the sample, inside the inner stainless steel shield. The measurement lines are all individually shielded in a coaxial cable geometry (except for the above-mentioned copper wires and for a short distance inside the shielded sample holder, between the input connector and the RC filters). The high-frequency filtering and 

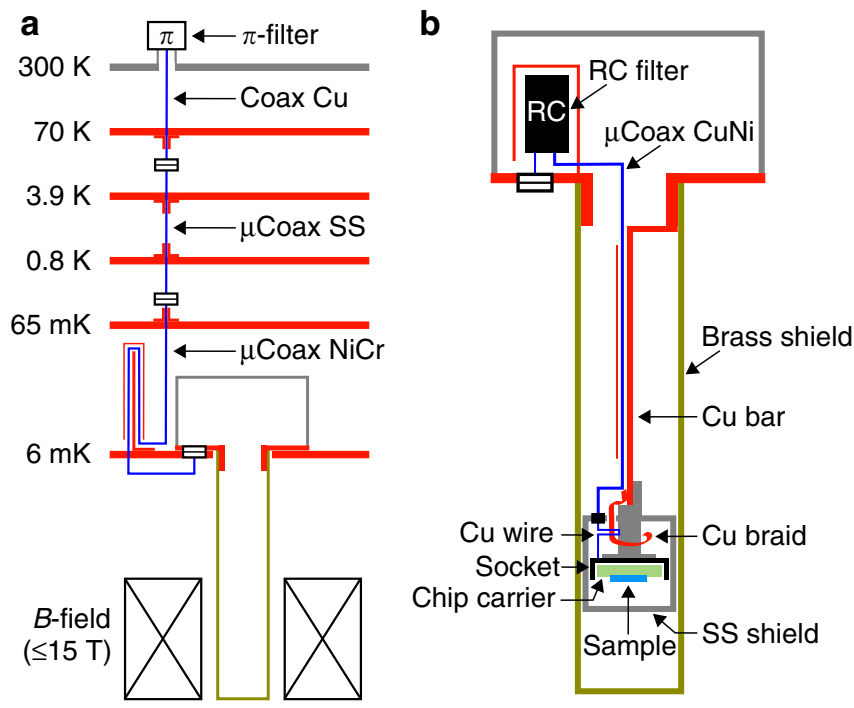

Figure 4 | Experimental set-up. (a) Diagrammatic representation of the electrical lines between room temperature $(\sim 300 \mathrm{~K})$ and the top-loaded sample holder at base temperature. (b) Schematic representation of the top-loaded sample holder.

initial thermalization to the mixing chamber plate of the electrical lines are performed with homemade resistive microcoaxes $(\mu \mathrm{Coax} \mathrm{NiCr} \text { in Fig. } 4 \mathrm{a})^{20}$. Because the electrical noise integrated over the full bandwidth needs to be smaller than a fraction of $\mu \mathrm{V}$, we only keep the bandwidth used for the measurements with personalized RC filters directly located inside the sample holder. This is most particularly important with a cryogen-free dilution refrigerator in the presence of a magnetic field because of the electrical noise induced by vibrations. We now compare the three investigated primary electronic thermometers.

Quantum shot noise thermometry stands out as the most robust and straightforward approach. It is based on simple physics, directly probes the temperature of the electrons through their energy distribution ${ }^{18}$ and does not require a separate calibration of the noise measurement set-up. The main possible artefact is local heating induced by the dissipated Joule power at finite dc bias. Such a heating typically scales linearly with $V_{\mathrm{dc}}$ (refs 44,45). It is therefore difficult to distinguish from a slight increase in the shot noise ${ }^{24,25}$. The present implementation in the quantum Hall regime, however, provides a strong protection against heating artefacts, owing to the spatial separation between incoming and outgoing currents. Although it is not necessary to determine the factor $\sum \tau_{n}\left(1-\tau_{n}\right)$ for the voltage-biased quantum conductor, it is important to make sure that it does not depend on $V_{\mathrm{dc}}$. For a single-channel quantum conductor, the dependence of $\tau(1-\tau)$ with voltage bias is minimized at $\tau \sim 0.5$, and $\tau\left(V_{\mathrm{dc}}\right)$ can be monitored simultaneously with the noise measurements. The main challenge with quantum shot noise thermometry is in the sensitivity of the noise measurement set-up; however, the associated temperature uncertainty can be statistically quantified. Note that the achieved resolution of $6.0 \pm 0.1 \mathrm{mK}$ is comparable to the accuracy of the provisional low-temperature-scale standard $(\text { PLTS-2000) })^{46}$

Coulomb blockade thermometry is also very straightforward and has the advantage of being less demanding on the measurement sensitivity. It is consequently widespread in the field of mesoscopic physics. However, the extracted temperature is easily/often artificially increased by charge fluctuations in the device vicinity, or by the electrical noise on the capacitively coupled gates. Such an artefact could be detected as a gate voltage-dependent increase in the noise level, proportional to $\partial G_{\mathrm{SET}} / \partial V_{\mathrm{g}}$, provided that a significant part of the charge fluctuations is within the measurement bandwidth. In general, the corresponding temperature increase is difficult to establish, except by comparing with another electronic thermometer. Here the agreement obtained with both the quantum shot noise and dynamical Coulomb blockade thermometers demonstrates a negligible artificial increase in the electronic temperature.

Dynamical Coulomb blockade thermometry can be difficult to use in general, if the surrounding circuit is not known a priori at relevant $\mathrm{GHz}$ frequencies. As in the case of quantum shot noise, a possible artefact is heating at a finite dc bias. This can be minimized by using a tunnel contact of very large impedance compared with the circuit. In contrast to quantum shot noise, the quantum Hall regime does not provide a protection against heating (in the central metallic island, for the dynamical Coulomb blockade experimental configurations). However, the very large renormalized tunnel resistance, 100 larger than the series resistance, ascertains negligible heating effects. Moreover, the dynamical Coulomb blockade thermometry is here particularly straightforward to implement because of the precise knowledge of the circuit.

With the consistent temperatures obtained by three primary thermometers, each relying on different physical mechanisms, we firmly established electronic thermometry standards in the regime of ultra-low temperatures. The achievement of $6 \mathrm{mK}$ electronic temperature, with the mesoscopic circuit in vacuum and using a medium-sized dilution refrigerator, provides a platform for further reduction of the temperature, using additional thermalization and cooling techniques ${ }^{15,47,48}$ towards the sub-millikelvin range.

\section{Methods}

Sample. The sample was nanostructured by standard e-beam lithography in a $\mathrm{Ga}(\mathrm{Al})$ As $2 \mathrm{DEG}$ of density $2.5 \times 10^{11} \mathrm{~cm}^{-2}$ and mobility $10^{6} \mathrm{~cm}^{2} \mathrm{~V}^{-1} \mathrm{~s}^{-1}$. The AuGeNi metallic island was diffused by thermal annealing into the semiconductor heterojunction to make an electrical contact of negligible resistance with the 2DEG (see Methods in ref. 8 for the electrical characterization of the contact in the same sample).

Measurement techniques. The differential conductance measurements were performed using standard lock-in techniques at frequencies below $200 \mathrm{~Hz}$ and using rms ac excitation voltages smaller than $k_{\mathrm{B}} T / e$. The sample was current-biased by a voltage source in series with a $100 \mathrm{M} \Omega$ polarization resistance at room temperature. The applied current was converted on-chip into a voltage independent of the device configuration by taking advantage of the well-defined quantum Hall resistance to an adjacent grounded electrode $\left(h / v e^{2}\right.$ at filling factor $v$ ). Similarly, the current transmitted across (reflected from) the device was converted into a voltage with the $h / v e^{2}$ quantum Hall resistance. The noise measurement set-up includes a homemade cryogenic pre-amplifier and an $L-C$ tank circuit of resonant frequency $0.84 \mathrm{MHz}$; see the online Supplementary Information and also the Supplementary Material of ref. 11 for a more detailed description.

Data availability. The data that support the findings of this study are available from the corresponding author upon request.

\section{References}

1. Willett, R. et al. Observation of an even-denominator quantum number in the fractional quantum hall effect. Phys. Rev. Lett. 59, 1776-1779 (1987).

2. Pan, W. et al. Exact quantization of the even-denominator fractional quantum hall state at $v=5 / 2$ landau level filling factor. Phys. Rev. Lett. 83, 3530-3533 (1999).

3. Dolev, M., Heiblum, M., Umansky, V., Stern, A. \& Mahalu, D. Observation of a quarter of an electron charge at the $v=5 / 2$ quantum hall state. Nature 452, 829-834 (2008)

4. Radu, I. P. et al. Quasi-particle properties from tunneling in the $v=5 / 2$ fractional quantum hall state. Science 320, 899-902 (2008).

5. Deng, N. et al. $v=5 / 2$ fractional quantum hall state in the presence of alloy disorder. Phys. Rev. Lett. 112, 116804 (2014). 
6. Potok, R. M., Rau, I. G., Shtrikman, H., Oreg, Y. \& Goldhaber-Gordon, D. Observation of the two-channel kondo effect. Nature 446, 167-171 (2007).

7. Keller, A. J. et al. Universal fermi liquid crossover and quantum criticality in a mesoscopic system. Nature 526, 237-240 (2015).

8. Iftikhar, Z. et al. Two-channel kondo effect and renormalization flow with macroscopic quantum charge states. Nature 526, 233-236 (2015).

9. Giazotto, F., Heikkilä, T., Luukanen, A., Savin, A. \& Pekola, J. Opportunities for mesoscopics in thermometry and refrigeration: physics and applications. Rev. Mod. Phys. 78, 217-274 (2006).

10. Meschke, M., Guichard, W. \& Pekola, J. P. Single-mode heat conduction by photons. Nature 444, 187-190 (2006).

11. Jezouin, S. et al. Quantum limit of heat flow across a single electronic channel. Science 342, 601-604 (2013).

12. Pekola, J. P. Towards quantum thermodynamics in electronic circuits. Nat. Phys. 11, 118-123 (2015).

13. Bradley, D. et al. Nanoelectronic primary thermometer below $4 \mathrm{mK}$. Nat. Commun. 7, 10455 (2016).

14. Xia, J. et al. Ultra-low-temperature cooling of two-dimensional electron gas. Phys. B 280, 491-492 (2000).

15. Samkharadze, N. et al. Integrated electronic transport and thermometry at millikelvin temperatures and in strong magnetic fields. Rev. Sci. Instrum. 82, 053902 (2011)

16. Chung, Y. C., Heiblum, M. \& Umansky, V. Scattering of bunched fractionally charged quasiparticles. Phys. Rev. Lett. 91, 216804 (2003).

17. Maradan, D. et al. GaAs quantum dot thermometry using direct transport and charge sensing. J. Low Temp. Phys. 175, 784-798 (2014).

18. Blanter, Y. M. \& Büttiker, M. Shot noise in mesoscopic conductors. Phys. Rep. 336, 1-166 (2000).

19. Grabert, H. \& Devoret, M. H. (eds) Single Charge Tunneling (Plenum, 1992)

20. Glattli, D. C., Jacques, P., Kumar, A., Pari, P. \& Saminadayar, L. A noise detection scheme with $10 \mathrm{mK}$ noise temperature resolution for semiconductor single electron tunneling devices. J. Appl. Phys. 81, 7350-7356 (1997).

21. Saminadayar, L., Glattli, D. C., Jin, Y. \& Etienne, B. Observation of the $e / 3$ fractionally charged laughlin quasiparticle. Phys. Rev. Lett. 79, 2526-2529 (1997).

22. dePicciotto, R. et al. Direct observation of a fractional charge. Nature 389, 162-164 (1997).

23. Reznikov, M., Heiblum, M., Shtrikman, H. \& Mahalu, D. Temporal correlation of electrons: suppression of shot noise in a ballistic quantum point contact. Phys. Rev. Lett. 75, 3340-3343 (1995).

24. Kumar, A., Saminadayar, L., Glattli, D. C., Jin, Y. \& Etienne, B. Experimental test of the quantum shot noise reduction theory. Phys. Rev. Lett. 76, 2778-2781 (1996).

25. Henny, M., Oberholzer, S., Strunk, C. \& Schönenberger, C. 1/3-shot-noise suppression in diffusive nanowires. Phys. Rev. B 59, 2871-2880 (1999)

26. Spietz, L., Lehnert, K. W., Siddiqi, I. \& Schoelkopf, R. J. Primary electronic thermometry using the shot noise of a tunnel junction. Science 300, 1929-1932 (2003).

27. Pistolesi, F., Bignon, G. \& Hekking, F. W. J. Subgap noise of a superconductornormal-metal tunnel interface. Phys. Rev. B 69, 214518 (2004).

28. Gabelli, J. \& Reulet, B. Shaping a time-dependent excitation to minimize the shot noise in a tunnel junction. Phys. Rev. B 87, 075403 (2013).

29. Landauer, R. Residual resistivity dipoles. Z. Phys. B 21, 247-254 (1975).

30. Anderson, P. W., Thouless, D. J., Abrahams, E. \& Fisher, D. S. New method for a scaling theory of localization. Phys. Rev. B 22, 3519-3526 (1980).

31. Büttiker, M. Four-terminal phase-coherent conductance. Phys. Rev. Lett. 57, 1761-1764 (1986).

32. Liang, Y. X., Dong, Q., Gennser, U., Cavanna, A. \& Jin, Y. Input noise voltage below $1 \mathrm{nV} / \mathrm{Hz}^{1 / 2}$ at $1 \mathrm{kHz}$ in the hemts at $4.2 \mathrm{~K}$. J. Low Temp. Phys. 167, 632-637 (2012)

33. Dong, Q. et al. Ultra-low noise high electron mobility transistors for high-impedance and low-frequency deep cryogenic readout electronics. Appl. Phys. Lett. 105, 013504 (2014).

34. Beenakker, C. W. J. Theory of coulomb-blockade oscillations in the conductance of a quantum dot. Phys. Rev. B 44, 1646-1656 (1991).

35. Jezouin, S. et al. Controlling charge quantization with quantum fluctuations. Nature 536, 58-62 (2016).
36. Altimiras, C., Gennser, U., Cavanna, A., Mailly, D. \& Pierre, F. Experimental test of the dynamical coulomb blockade theory for short coherent conductors. Phys. Rev. Lett. 99, 256805 (2007)

37. Parmentier, F. D. et al. Strong back-action of a linear circuit on a single electronic quantum channel. Nat. Phys. 7, 935-938 (2011).

38. Mebrahtu, H. T. et al. Quantum phase transition in a resonant level coupled to interacting leads. Nature 488, 61-64 (2012).

39. Jezouin, S. et al. Tomonaga-luttinger physics in electronic quantum circuits. Nat. Commun. 4, 1802 (2013).

40. Joyez, P. \& Esteve, D. Single-electron tunneling at high temperature. Phys. Rev. B 56, 1848-1853 (1997).

41. Anthore, A., Pierre, F., Pothier, H. \& Esteve, D. Magnetic-field-dependent quasiparticle energy relaxation in mesoscopic wires. Phys. Rev. Lett. 90, 076806 (2003).

42. Odintsov, A. A., Falci, G. \& Schön, G. Single-electron tunneling in systems of small junctions coupled to an electromagnetic environment. Phys. Rev. B 44, 13089-13092 (1991)

43. Mebrahtu, H. T. et al. Observation of majorana quantum critical behaviour in a resonant level coupled to a dissipative environment. Nat. Phys. 9, 732-737 (2013).

44. Smith, C. G. \& Wybourne, M. N. Electric field heating of supported and free-standing AuPd fine wires. Solid State Commun. 57, 411-416 (1986).

45. Wellstood, F. C., Urbina, C. \& Clarke, J. Hot-electron effects in metals. Phys. Rev. B 49, 5942-5955 (1994).

46. Rusby, R. L. et al. The provisional low temperature scale from $0.9 \mathrm{mK}$ to $1 \mathrm{~K}$, plts-2000. J. Low Temp. Phys. 126, 633-642 (2002).

47. Clark, A. C., Schwarzwälder, K. K., Bandi, T., Maradan, D. \& Zumbühl, D. M. Method for cooling nanostructures to microkelvin temperatures. Rev. Sci. Instrum. 81, 103904 (2010).

48. Casparis, L. et al. Metallic coulomb blockade thermometry down to $10 \mathrm{mK}$ and below. Rev. Sci. Instrum. 83, 083903 (2012).

\section{Acknowledgements}

This work was supported by the European Research Council (ERC-2010-StG-20091028, no. 259033), the French RENATECH network, the national French programme 'Investissements d'Avenir' (Labex NanoSaclay, ANR-10-LABX-0035) and the European Seventh Framework Program (EU FP7, no. 263455).

\section{Author contributions}

Measurements and analysis: F.P. with inputs from Z.I.; low temperature set-up: F.P. with inputs from A.A. and S.J.; noise measurement set-up: F.D.P., S.J., A.A. and F.P. HEMT nanofabrication: Y.J.; heterojunction growth: A.C., A.O. and U.G.; sample nanofabrication: F.D.P. and A.A; manuscript preparation: F.P. with inputs from A.A. and U.G.; project planning and supervision: F.P.

\section{Additional information}

Supplementary Information accompanies this paper at http://www.nature.com/ naturecommunications

Competing financial interests: The authors declare no competing financial interests.

Reprints and permission information is available online at http://npg.nature.com/ reprintsandpermissions/

How to cite this article: Iftikhar, Z. et al. Primary thermometry triad at $6 \mathrm{mK}$ in mesoscopic circuits. Nat. Commun. 7: 12908 doi: 10.1038/ncomms12908 (2016). International License. The images or other third party material in this article are included in the article's Creative Commons license, unless indicated otherwise in the credit line; if the material is not included under the Creative Commons license, users will need to obtain permission from the license holder to reproduce the material. To view a copy of this license, visit http://creativecommons.org/licenses/by/4.0/

(C) The Author(s) 2016 


\section{Appendix E}

\section{Experimental procedures}

In the first section of this appendix, we explain the method we used to extract the conductance of the whole device from voltage measurements. The in situ conductance of a single QPC can also be extracted when none of the three QPCs is completely pinched off.

The second section discusses the tuning of the device to observe 'charge' Kondo effect and the methods used to extract the conductance at energy level degeneracy. In the last section we explain the systematic treatment of large sets of measurements and we discuss some experimental artifacts to handle.

\section{E.1 Extraction of in situ conductances}

Irrespectively of the configuration of the QPCs and other surface gates, the resistance of the sample 1 is fixed by IQHE to $1 /\left(v G_{K}\right)$. We use lock-in amplifiers to measure the voltages defined in Fig. E.1. At first, we will assume that the voltage are measured without any calibration offset and that all the measurement lines are equivalent (same gain). In the second subsection, we will explain how to calibrate the gain and the offsets.

\section{E.1.1 Conductance formulae}

We assume that the electronic channels are completely reflected on the characterization gates $\left(\tau_{\mathrm{sw}_{1}}=\tau_{\mathrm{sw}_{2}}=0\right.$, uncolored in Fig. E.1) and that only the outermost channels have partial transmission $\tau_{i} \leq 1$ (otherwise the formulae would be differ-

\footnotetext{
${ }^{1}$ One should distinguish the conductance of the sample, which means 'between a given input port of the circuit and the ground', from the conductance of the device, which means 'between an input port and an output port'. The latter depends on the configuration of the surface gates.
} 


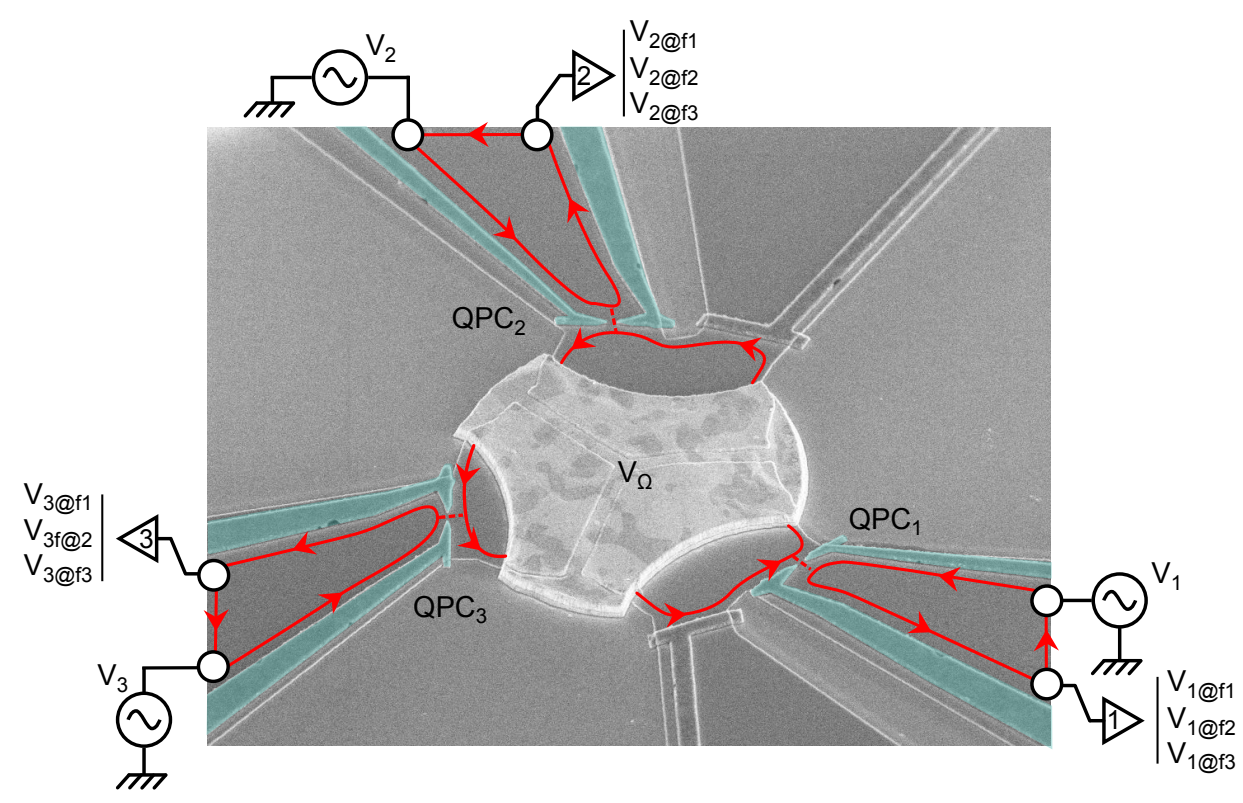

Figure E.1: Measurement schematics. We use three low frequency a.c. voltage sources (labeled $V_{j}$ ) to inject the signal in the IQHE edge channels (in red). Lockin amplifiers are used to measure simultaneously nine voltages a.c. signals. The voltage measured at the point $i$ in response to the voltage $V_{j}$ injected "at frequency $j$ " is labeled $V_{i @ f j}$.

ent). As the current is conserved, the injected voltage $V_{j}$ is the sum of the voltages measured "at frequency $j$ ":

$$
V_{j}=\sum_{i=1}^{3} V_{i @ f j}
$$

There are basically two useful formulas. Either one considers the current transmitted through the island (voltages as $V_{i @ f j}$, with $i \neq j$ ) or the current reflected at $\mathrm{QPC}_{i}$ (the voltage $\left.V_{i @ f i}\right)$.

Applying the Kirchoff's current law successively on contact $i$ and on the central ohmic contact $\Omega$ yields:

$$
\nu G_{K} V_{i @ f j}=G_{i} V_{\Omega @ f j}=\frac{G_{i} G_{j}}{G_{1}+G_{2}+G_{3}} V_{j}
$$

where $V_{\Omega @ f j}$ is the voltage on the central ohmic contact measured at the frequency of the source $V_{j}$. To get the formula for the current in reflection, we just add the contribution of the inner quantum Hall channels: 


$$
v G_{K} V_{i @ f i}=G_{i} V_{\Omega @ f i}+\left(v G_{K}-G_{i}\right) V_{i}=\left(v G_{K}-G_{i}\left(1-\frac{G_{i}}{G_{1}+G_{2}+G_{3}}\right)\right) V_{i}
$$

We can therefore extract the in situ conductances $G_{i}$ of each QPC from the voltage measurements. Measurements of reflected and transmitted signals are redundant, because of current conservation. Thus we define the following "rescaled voltages" $G_{i i}$ (that have the dimension of a conductance) and that allow for a direct averaging of the reflected and transmitted signals:

$$
G_{i i} \triangleq v G_{K}\left(1-\frac{V_{i @ f i}}{V_{i}}\right)=v G_{K} \frac{\sum_{j \neq i} V_{j @ f i}}{V_{i}}
$$

This measured conductance $G_{i i}$ depends on the in situ conductances as:

$$
G_{i i}=\frac{\sum_{j \neq i} G_{j} G_{i}}{G_{1}+G_{2}+G_{3}}
$$

Once we have measured the $G_{i i}$ for all $i=\{1,2,3\}$, we can invert the system and find the in situ conductances $G_{1}, G_{2}$ and $G_{3}$. The single assumption required to extract the in situ conductances is the validity of the Kirchoff's law on each contact of the nanofabricated sample.

Note that if one of the three QPCs is completely pinched off (say $G_{k}=0$ ), we can only access to the serial conductance $G_{i} G_{j} /\left(G_{i}+G_{j}\right)$ and cannot extract the individual conductances $G_{i}$ and $G_{j}$.

\section{E.1.2 Calibration of the gain and the offset}

We use the same kind of voltage sources, pre-amplifiers and lock-in amplifiers in all the measurement setup. However, in practice, the gain on each measurement line may slightly differ and a systematic offset can exist.

\section{Offset}

In order to calibrate the offset, one just needs to measure all the voltages $V_{i @ f j}$ for a plugged and unplugged voltage source. This is done with all the QPCs completely pinched off, therefore, by this most accurate procedure, we cannot calibrate the offset on the $V_{i @ f i}$ measurements (in reflection). We found the same typical offset of $\sim-7 \times 10^{-11} \mathrm{~V}$ on all the voltages $V_{i @ f j}$ (with $i \neq j$ ).

For comparison, the voltage applied on the sample at base temperature $T \approx$ $7.9 \mathrm{mK}$ is $V_{\text {a.c. }} \approx 4.8 \times 10^{-7} \mathrm{~V}$ (which is of the order of the thermal energy that corresponds to a voltage of $k_{B} T / e \approx 6.8 \times 10^{-7} \mathrm{~V}$ ). This offset becomes important 
when measuring weak signals, when one of the conductances is very small (see Fig. 4.10 on page 88 .

\section{Gain}

In Table B.2 we have shown that, on the first quantum Hall plateau, the transmission of the outermost channel to the central ohmic contact is perfect within our accuracy. That measurement was independent of any gain calibration because we used ratio of voltages from the same measurement line (in reflection $V_{i @ f i}$ ).

In order to calibrate the gain of the measurement lines, we set all the QPCs to transmission $\tau_{1}=\tau_{2}=\tau_{3}=1$. We assume these transmissions to be exactly equal to one as previously checked. Note that for this calibration, although the calibrated offsets can be taken into account, they are negligible since the signals are relatively large. In this configuration, the ratio of measured voltages (e.g. $V_{1 @ f 3} / V_{2} @ f 3$ ) directly give ratio of gains (e.g. $\gamma_{1} / \gamma_{2}$ at "frequency 3 ", where $\gamma_{i}$ is the gain on the $i$-th measurement line).

We noticed that, the gains $\gamma_{1} / \gamma_{2}=1.0018$ and $\gamma_{3} / \gamma_{2}=1.0095$ (with $\gamma_{2}=1$ taken as reference) yield the same $G_{i i}$ for both the measurements in reflection and in transmission: $\left(1-V_{i @ f j} / V_{i}\right) /\left(\left(V_{j @ f i}+V_{k} @ f i\right) / V_{i}\right)=1 \pm 5 \times 10^{-5}$. When considering our three frequencies $f_{1}=145 \mathrm{~Hz}, f_{1}=163 \mathrm{~Hz}$ and $f_{1}=185 \mathrm{~Hz}$, the typical variation of a gain ratio with frequency $\left(\sim 4 \times 10^{-3}\right)$ is within the uncertainty bar of the gain calibration.

\section{E.2 Device tuning to measure the multi-channel 'charge' Kondo effect}

In order to observe the flow of the conductance towards the $N$-channel 'charge' Kondo fixed point, one needs to set symmetric transmission $\tau_{1}=\tau_{2}=\ldots=\tau_{N}$ of $N$ electronic channels and also to tune the voltage gate $V_{g}$ at charge degeneracy $\delta V_{g}=0$.

\section{E.2.1 Symmetric coupling}

Our goal is to know the voltage to apply on each QPC to have the same transmission $\tau \triangleq \tau_{1}=\tau_{2}=\tau_{3}$, and we want a dozen of values picked in the range $\tau \in[0,1]$.

\section{Approximate tuning}

The first step is to use the characterization gates to measure the intrinsic transmission (in DCB regime at large voltage bias). Then we get the voltage $V_{\mathrm{qpc}_{i, k}}$ to apply 
on $\mathrm{QPC}_{i}$ to get $\tau_{i, k}$ (where $\tau_{i, k}$ refers to the $k$-th picked up value on $\mathrm{QPC}_{i}$ ).

We know the correction to apply on $V_{\mathrm{qpc}_{i}}$ to go from the DCB regime to the configuration where all the switches are at $\tau_{\mathrm{sw}}=0$ (see Appendix B.1.4). We have determined each $V_{\mathrm{qpc}_{i, k}}$ while the other QPCs were in a reference configuration $\left(\tau_{j \neq i}=0\right)$, but we also know the correction to apply on $V_{\mathrm{qpc}_{i}}$ when we change the voltage on the other QPCs.

It means that we are able to measure Coulomb oscillations with all the QPCs having the same transmission: $\tau_{1}=\tau_{2}=\tau_{3}$.

\section{Fine tuning}

When none of the three QPCs is completely pinched off $\left(\tau_{1,2,3} \neq 0\right)$, we are able to find the three individual conductances $G_{i}$. We want to verify whether the in situ conductances are symmetric $G_{1}=G_{2}=G_{3}$ for the multi-channel Kondo experiment. In this case, there are Coulomb oscillations and we focus on maxima of the conductances $G_{i}\left(\delta V_{g} \approx 0\right)$ where the signal is maximal.

The procedure explained in the previous subsection relied on several capacitive crosstalk calibrations which limits our accuracy. We therefore perform an additional fine adjustment of the QPC symmetry.

For this fine symmetry tuning, we use $\mathrm{QPC}_{2}$ as a reference at the temperature $T \approx 18 \mathrm{mK}$, and we adjusted the two others to have all the in-situ conductances exactly symmetric. After this fine tuning we have noticed that the values of $V_{\mathrm{qpc}_{i, k}}$ have been shifted by $\sim 0.2 \mathrm{mV}$ for $\mathrm{QPC}_{1}$ and by $\sim 0.4 \mathrm{mV}$ for $\mathrm{QPC}_{3}$. These shifts are compatible with the uncertainty intervals on the crosstalk calibrations.

\section{Approximate transmission symmetry requirement}

The effect of an asymmetry between the transmissions is visible on the renormalization flow diagram shown in Fig. 4.9 where we used the transmissions $\tau$ characterized in Fig. B.6. The difference between two successive transmissions ranges from 0.1 down to 0.03 in the vicinity of the $3 \mathrm{CK}$ fixed point where the step is finer. Even such a small difference has a dramatic effect on the conductance renormalization flow (see the criticality of the symmetry on the first off-diagonal arrows of Fig. 4.9. The requirement on the transmission symmetry to flow towards the right fixed point is therefore more restrictive than $\delta \tau<0.03$.

\section{E.2.2 Transmissions in practice}

\section{Dependence in energy $\tau(E)$}

We tried to minimize this dependence by choosing the best operating points for the QPCs (e.g. see Fig. A.5). The relative deviation $\Delta \tau / \tau$ between the transmission 
at equilibrium and the transmission at large voltage bias is typically of $\approx 10 \%$, it is maximal in the tunnel case $\tau \ll 1$ (see Fig. B.5).

\section{Stability of the QPCs}

The data acquisition of the multi-channel Kondo effect measurement took several months (because of many tests, calibrations, temperature measurements and other time consuming activities). We are able to correct for rare and small shifts of the QPCs. Before all our Kondo effect measurements (that take typically a night or a weekend), we perform a fine measurement of the intrinsic transmission $\tau\left(V_{\mathrm{qpc}}\right)$ of each QPC and adjust for a possible small $V_{\mathrm{qpc}}$ shift with respect to a reference measurement. And after the measurement, we redo a fine measurement of the transmissions to check if the QPCs were stable during the acquisition (if they were not, we redo the measurement).

\section{E.2.3 Tuning to charge degeneracy $\delta V_{g}=0$}

In order to increase our precision on the conductance at charge degeneracy $G\left(\delta V_{g}=\right.$ 0 ), we fit the conductance peak to a model. However, there is no prediction for the general shape of the conductance peak at arbitrary transmissions $\tau_{i}$ and temperature $T$.

For instance, in the two-channel case, theory predict a shape as $f\left(V_{g}\right) \propto$ $\left(\alpha V_{g} / k_{B} T\right) / \sinh \left(\alpha V_{g} / k_{B} T\right)$ in the tunnel regime $\tau \ll 1$ and a sinusoidal shape in the strong coupling regime $1-\tau \ll 1$. Therefore, we use a general model and extract $G\left(\delta V_{g}=0\right)$ in several steps to avoid any systematic error due to the specificity of the fitting function. Here follows the procedure used for the $3 \mathrm{CK}$ measurements.

\section{Raw data and approximate tuning}

In the 3CK measurement, we have three conductances to extract (whereas in 2CK we have only one). We apply no voltage bias $V_{\mathrm{dc}}$, the maximum of the conductance appears therefore at the same gate voltage $\delta V_{g}=0$ for all the signals. Thus we start with averaging the three signals $G_{\text {avg }} \triangleq\left(G_{11}+G_{22}+G_{33}\right) / 3$, an example is shown in Fig. E.2. The peaks are periodic and we can extract the highest value of each period. We use these maxima as a starting position for the fits.

\section{Large, intermediate and fine fit of the averaged data}

In this subsection, we are only interested on the position of the maxima of the conductance. This procedure is applied to each peak of $G_{\text {avg individually. We }}$ 


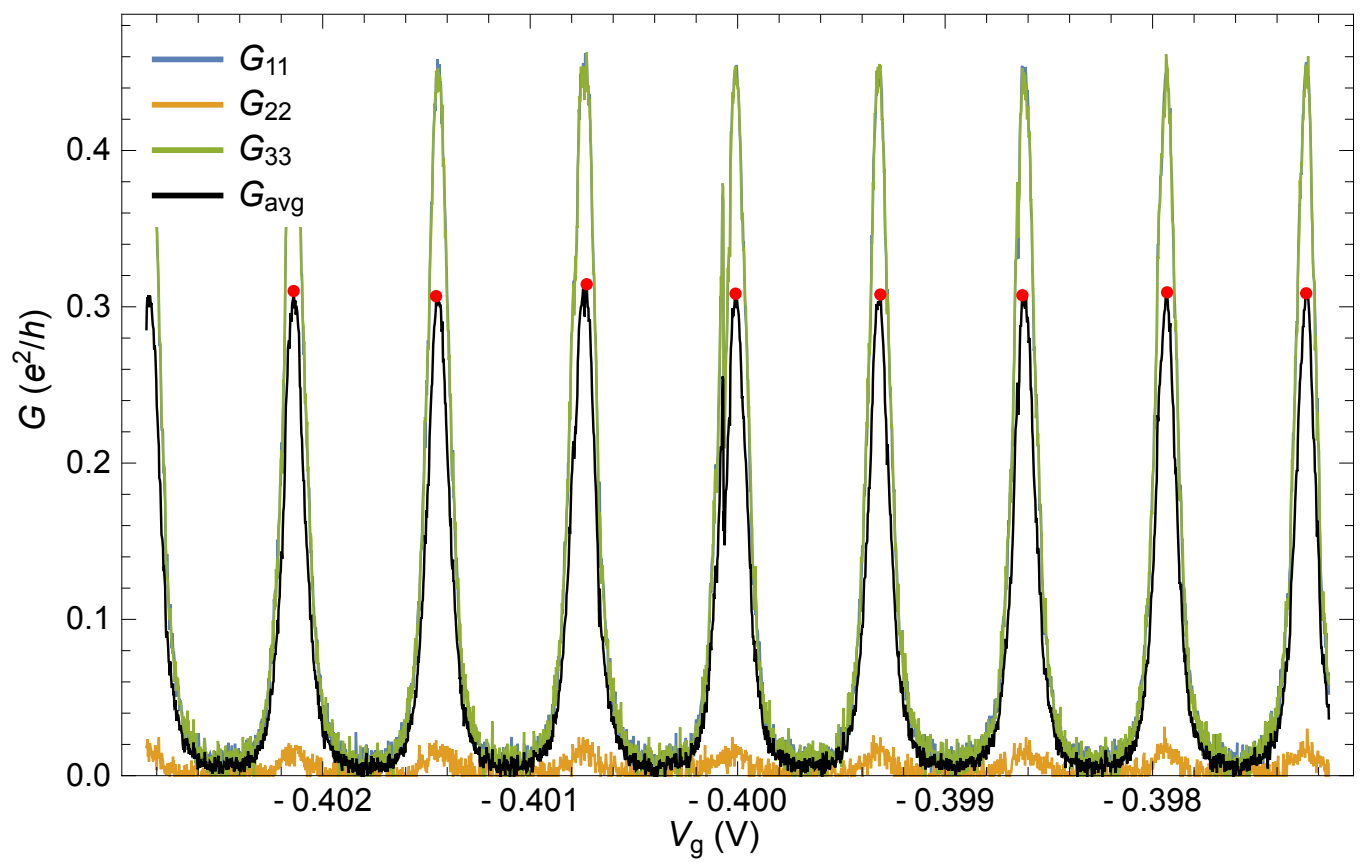

Figure E.2: Raw experimental data. The conductance $G_{i i}$ are plotted versus the gate voltage $V_{g}$ at $T \approx 7.9 \mathrm{mK}$ for $\tau_{1} \approx \tau_{3} \approx 0.1$ and $\tau_{2} \approx 0.79$. Because of the symmetry $\tau_{1} \approx \tau_{3}$, the signals $G_{11}$ and $G_{33}$ are superimposed. The black trace $G_{\text {avg }}$ correspond to the average of the three signals. The red dots show the local maxima of $G_{\text {avg }}$.

will determine the value of the conductance maxima $G_{i i}\left(\delta V_{g}=0\right)$ in the next subsection.

We use the following model based on the Airy function and that have four parameters (an offset $m$, an amplitude $\Delta$, a finesse $F$ and an origin $x_{0}$ ):

$$
m^{2}+\Delta^{2}\left(1-\frac{\left(1+F^{2}\right) \sin ^{2}\left(k\left(x-x_{0}\right)\right)}{1+F^{2} \sin ^{2}\left(k\left(x-x_{0}\right)\right)}\right)
$$

where $x$ is the variable and $k$ is replaced by $\pi / \overline{\Delta x}$, where $\overline{\Delta x}$ is the average distance between the peaks (which is proportional to $1 / C_{g}$ the capacitance of the plunger gate, in practice $\Delta V_{g}=0.70 \mathrm{mV}$ ). The parameter $F$ gives the finesse of the peak (the model is sinusoidal when $F=0$ and sharp when $F$ is large).

We will fit the experimental data in three steps. A 'large fit' is used to get an estimation of the parameters. A 'intermediate fit' will be performed on a smaller interval near the maximum to find its position $\left(\delta V_{g}=0\right)$. And finally, we use a 'fine fit' on a tiny region with only $\Delta$ as parameter. This last fit gives the value of the conductance at degeneracy independently of the model (since it has been done on a tiny region near the maximum, see Fig. E.3. 


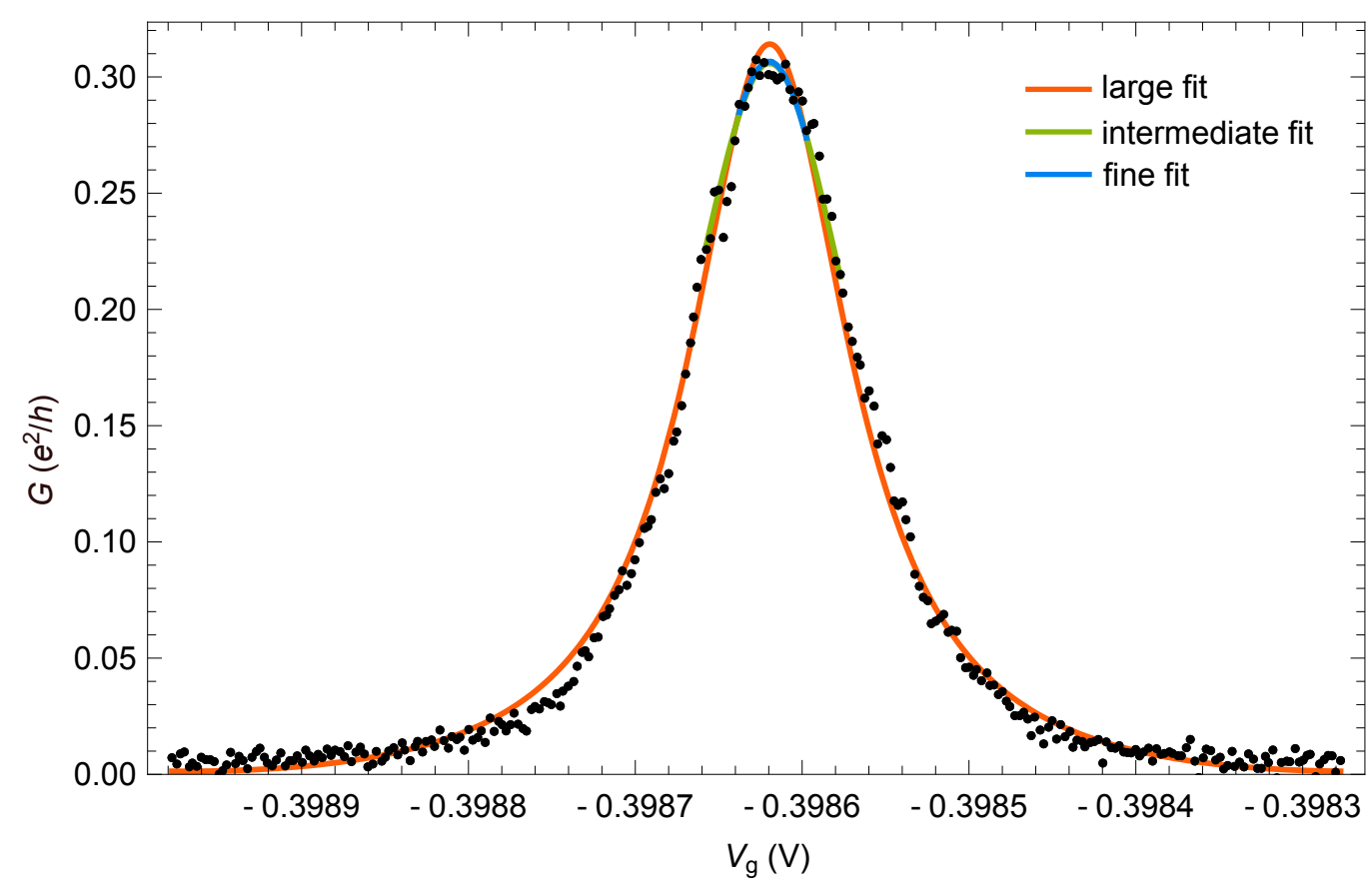

Figure E.3: Fit of experimental data in three steps. We focus on the third peak (from the right) of Fig. E.2. The three successive fits are performed on different ranges (colored traces).

The offset $m$ is no longer a parameter in the second fit (called 'intermediate'). Indeed, as the fit is done on a narrower range, the minimum of the oscillations could not be fitted. We set it to the mean value of the set of $m$ found at first fit.

Similarly, the finesse $F$ is not fitted in the 'fine fit' because letting $F$ free when fitting on a tiny region is not a good idea since the global curvature of the peak is not obvious. In this case, we use the median value rather than the mean to avoid wrong values that may happen sometimes because of experimental artifacts (see Fig. E.6 in Appendix E.3.

\section{Conductances at $\delta V_{g}=0$}

The last fit (the 'fine' one) yields the value of the conductance at degeneracy. We are not interested in this value for $G_{\text {avg }}$. However, we know the position of the degeneracy point $\delta V_{g}=0$ (which is given by the parameter $x_{0}$ ) for each peak.

To get the individual conductances, we fit again each individual signal $G_{i i}$ using only the 'large' and the 'fine' fits because we just need $m$ and $\Delta$ (see Fig. E.4). Indeed, $F$ is essentially the same for the three signals and the 'fine' fit is made on a narrow $\delta V_{g}$ interval such that the result does not depend on this fitting parameter. Finally we deduce the in situ conductances $G_{i}$ at degeneracy from the value of $G_{i i}$ 


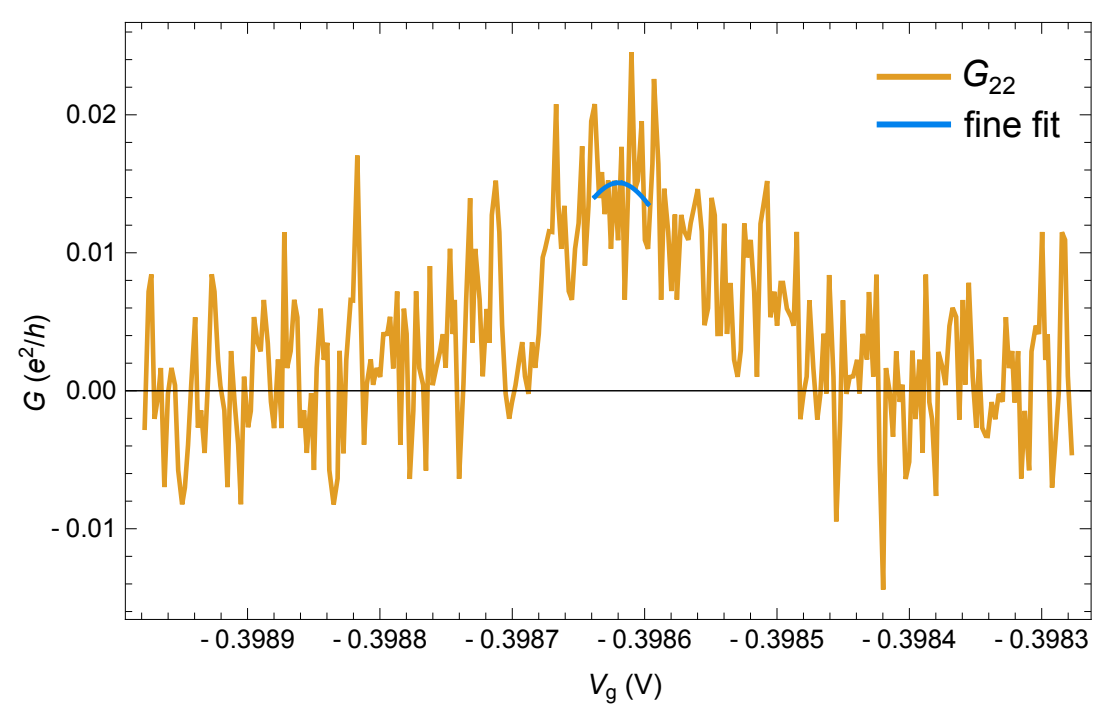

Figure E.4: Fine fit of a weak signal. The 'fine fit' of the weakest signal of Fig. E.2 $\left(G_{22}\right.$, in orange $)$ is shown with the corresponding experimental data.

using Eq. (E.3).

\section{Precision and accuracy}

The precision on the determination of $G_{i i}\left(\delta V_{g}=0\right)$ depends on the range over which the fit is performed. This precision increases as $\sqrt{N_{\text {points }}}$, where $N_{\text {points }}$ is the number of points in the 'fine fit' range. We choose a range that is a function of the finesse $F$ (the wider the peak, the larger the fit range). This has been adjusted to have 'fine fit' ranges small enough, not to be sensitive to the fitting function Eq. (E.4).

In order to increase the accuracy on $G_{i i}\left(\delta V_{g}=0\right)$, one has to acquire more peaks of a given configuration of transmissions and temperature (e.g. by averaging on all the peaks measured in Fig. E.3). However, one should be careful with respect to some experimental artifacts, as we will see in the next section.

\section{E.3 Experimental artifacts}

\section{E.3.1 Systematic peak selection}

\section{A typical example}

Our SET-like device is sensitive to the charge of the environment. We have noticed that some irregular pattern can appear on the Coulomb oscillations. When 
the transmissions and the temperature are fixed, sweeping the plunger gate $V_{g}$ should give periodic oscillations with a constant amplitude. In Fig. E.5, we show a typical artifact: a dip in the amplitude of the conductance peak around a specific gate voltage $V_{g} \approx-0.398 \mathrm{~V}$.

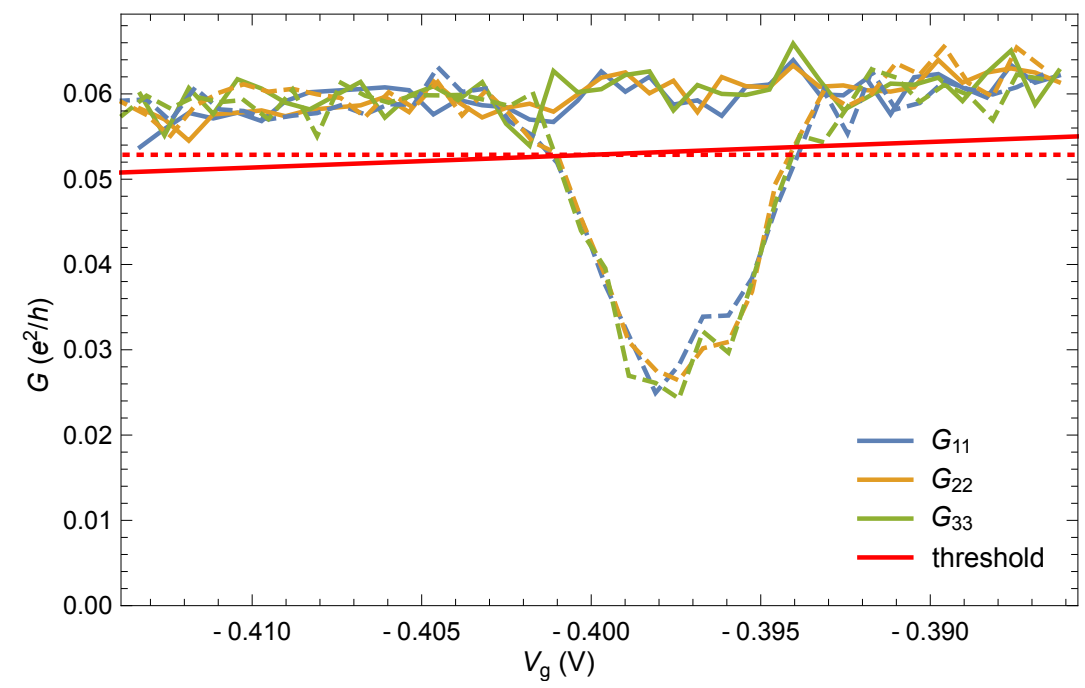

Figure E.5: Experiment artifact. The conductance at degeneracy $G_{i i}\left(\delta V_{g}=0\right)$ is plotted as a function of $V_{g}$ at $T \approx 12 \mathrm{mK}$ and for a symmetric tunnel configuration $\tau_{1}=\tau_{2}=\tau_{3} \approx 0.1$. Note that each sweep contains about 40 peak, only the conductance at degeneracy is plotted. The conditions were the same for all the curves, indeed the measurement program has just looped back to the first configuration between the evening (solid lines) and the morning (dashed lines). A systematic method excludes the points below the threshold given by the red line (see text in Appendix E.3.1 for the dotted line, and Appendix E.3.2 for the solid line).

The known mechanisms (e.g. charge fluctuations) for such artifact can only reduce the conductance. Therefore, we performed a statistical study and exclude the peaks that are anomalously low (this is also true for the $3 \mathrm{CK}$ measurements above the 3CK fixed point).

\section{Systematic exclusion}

The procedure to exclude the pathological peaks has been automatized because of the large quantity of data (and also to avoid a bias due to human intervention).

At a given temperature, the injected a.c. signal to measure the conductances is fixed, thus the noise $\sigma_{G}$ on the normalized measured voltage $G_{i i}$ is also fixed (whatever the configuration of the QPCs or the plunger gate $V_{g}$ )

For each sweep of $V_{g}$ (at $\tau$ fixed) we have a set of conductances at degeneracy that has a typical noise of $\sigma_{G}$. In each set, we exclude the points that are $n \times \sigma_{G}$ 
below the maximal value (see the red dotted line in Fig. E.5, which is plotted with $n=6$ ). Assuming a gaussian distribution of the points, this procedure with $n=6$ excludes less than $1 \%$ of normally distributed points (i.e. it essentially excludes only anomalous data). Note that the threshold $n \times \sigma_{G}$ should be weighted with $\sqrt{N_{\text {points }}}$, the number of points used in the fit of a peak.

About 6000 peaks have been analyzed to display the data shown in Chapter 3 (symmetric couplings $\Delta \tau=0$ ), which means 27 peaks in average for each configuration (of transmission and temperature).

\section{E.3.2 Crosstalk of the plunger gate}

In the $2 \mathrm{CK}$ and $3 \mathrm{CK}$ measurements, we have used $V_{\mathrm{sw} 2}$ as plunger gate. This voltage was swept around a value where we are sure that $\tau_{\mathrm{sw} 2}=0$ on a typical span of $V_{\mathrm{sw} 2} \in[-0.405 \mathrm{~V},-0.395 \mathrm{~V}]$.

Although this span is very small, we can correct the influence of this sweep on the transmission $\tau_{i}$ of the QPCs during oscillation measurement. If $V_{\mathrm{sw} 2}$ is crescent (going to positive values), the transmissions will be slightly higher at the end of sweep than at its beginning. This is visible on the conductances shown in Fig. E.5.

We have observed and calibrated this effect at high temperatures (at low temperature, as in Fig. E.5, the signal-to-noise ratio is not high enough to precisely calibrate such a small influence). We have averaged the slopes measured at $T \approx 55,80$ and $100 \mathrm{mK}$ and used the averaged slope to correct the data measured at all the temperature. One can compare the red solid line threshold in Fig. E.5 that takes into account the crosstalk correction (which is calibrated at $V_{g_{0}}=-0.4 \mathrm{~V}$ ) with the red dotted line which is plotted without correction.

\section{E.3.3 Averaging the selected peaks}

In Chapter 4, we study the conductance out of degeneracy $\delta V_{g} \neq 0$. The peaks we presented are averaged only on the data selected by the systematic procedure described above, in Appendix E.3.1.

Sometimes, when sweeping the plunger gate $V_{g}$, there is a jump in the conductance Coulomb oscillations (see figure Fig. E.6). We eliminate manually the rare affected peaks. 


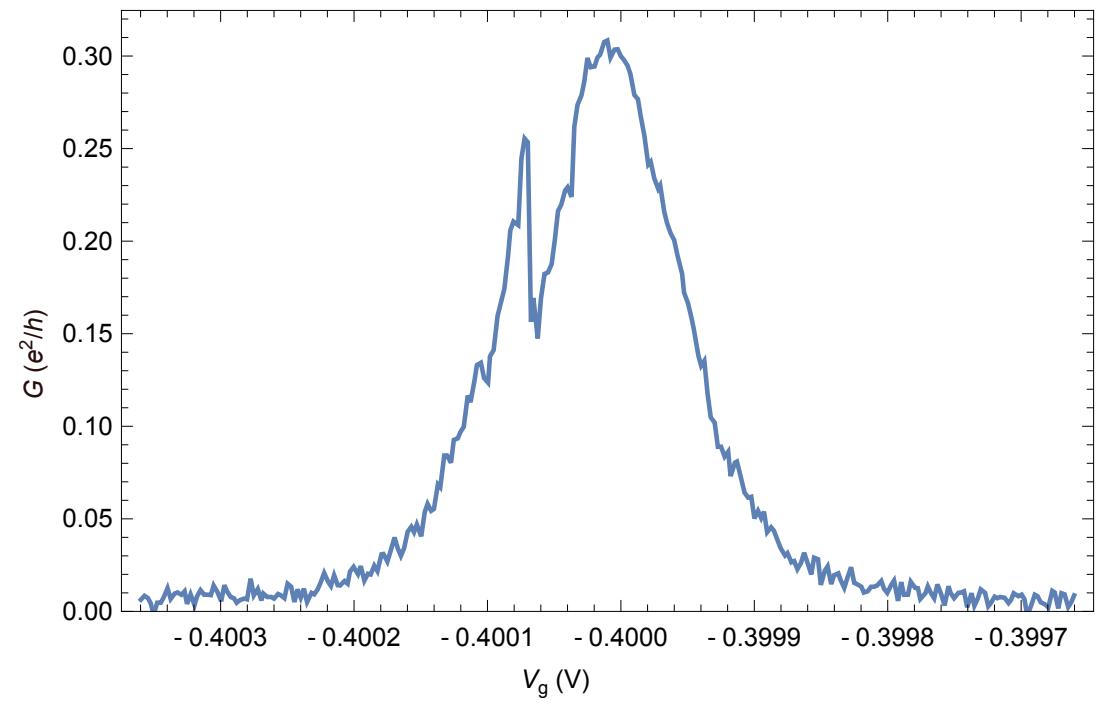

Figure E.6: Instability of the charge on the island. This instability has been measured on the fourth peak (from the left) displayed in Fig. E.2 


\section{Appendix F}

\section{Résumé en français}

Cette thèse traite de mesures de transport électrique effectuées sur un nanodispositif caractérisable et modifiable in situ (montré en Fig. F.1). Grâce à sa structure hybride métal-semiconducteur, ce dispositif fournit un banc d'essai quantitatif pour la physique des électrons fortement corrélés et celle des phénomènes critiques. Il peut être placé dans un régime où peu de canaux électroniques indépendants sont en forte interaction avec les degrés de liberté quantiques macroscopiques de la 'charge' du nœud métallique (en violet sur la Fig. F.1) du circuit. Nous utilisons cet échantillon pour sonder et contrôler le degré de quantification d'un nœud métallique en fonction de sa connexion à d'autres conducteurs. Ce dispositif peut aussi être ajusté pour implémenter le modèle Kondo multi-canaux et réaliser des simulations quantiques fiables de ce modèle quantique à N corps. Ainsi, il fournit un banc d'essai à certaines des plus puissantes méthodes de physique quantique à $\mathrm{N}$ corps (le group de renormalisation numérique [131, 20, 113], l'ansatz de Bethe [117, 13], la théorie des champs conformes [116, 3, 4] ou la bosonisation [39, 78, 43]).

Nous avons observé de bons accords entre nos données expérimentales et les lois de puissance universelles prédites à la fois proche de la limite de couplage fort dans le problème de la quantification de la charge et au voisinage des points critiques quantiques de l'effet Kondo multi-canaux. La transition depuis ces points critiques révèle une physique intrigante, en particulier à température intermédiaire. L'approche que nous développons dans cette thèse ouvre la voie à des études plus approfondies de la fascinante physique non-Fermi liquide qui est sous-jacente au domaine des matériaux fortement corrélés.

Le reste de ce chapitre donne un aperçu rapide des résultats obtenus au cours de cette thèse, chaque section correspondant à un chapitre du manuscrit. Nous commençons par une description de notre dispositif "à un électron" et nous répondons à une question relativement ancienne concernant le critère de destruction de la quantification de la charge par les fluctuations quantiques dans ce genre de disposi- 


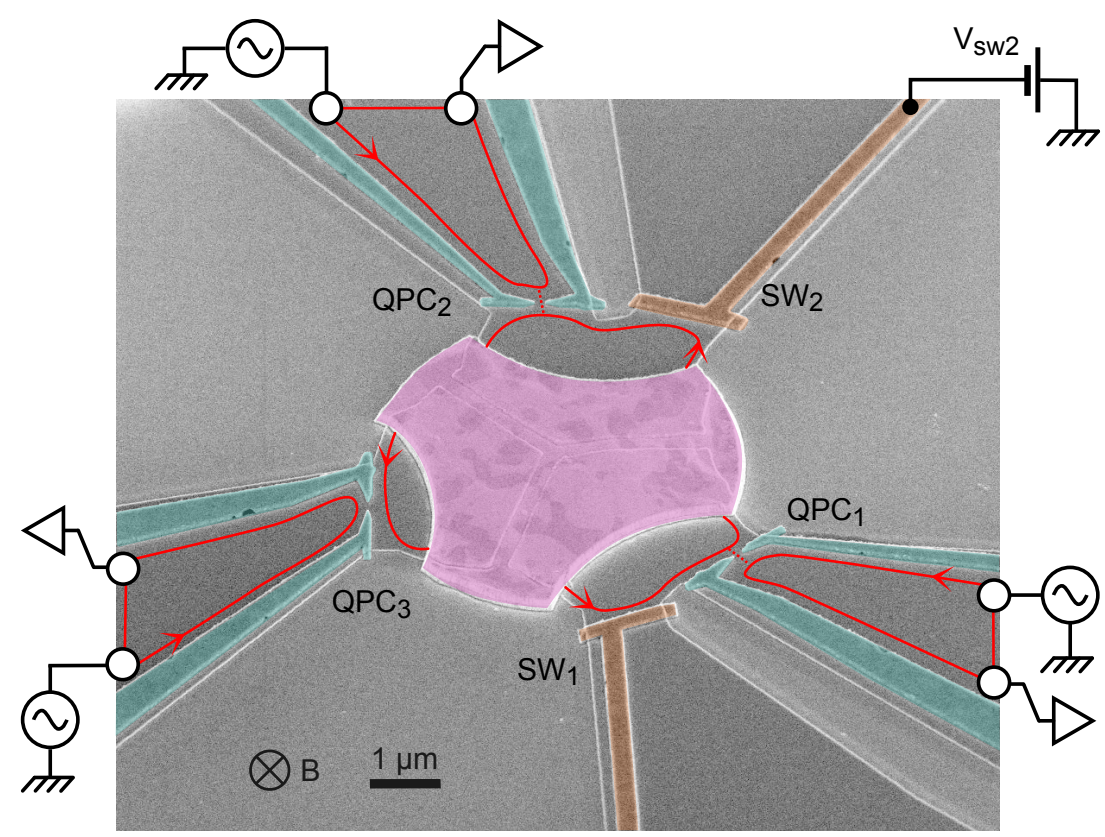

Figure F.1: Image de l'échantillon en fausses couleurs prise au microscope électronique à balayage. Cette figure montre le contact ohmic central (en violet) qui redistribue le courant $\mathrm{AC}$ injecté par des sources de tension (en dehors de l'image) dans les canaux chiraux de l'effet Hall quantique entier (lignes rouges) par l'intermédiaire de larges contacts ohmiques (cercles blancs) qui ne sont pas montrés dans l'image. Le signal basse fréquence est mesuré par des Lock-in (triangles). Cet échantillon compte trois contacts ponctuels quantiques ( QPCs, en cyan) et deux interrupteurs (en orange) utilisés pour la caractérisation. La valeur de transmission des QPCs et l'état des interrupteurs sont contrôlés par les sources de tension qui connectent (disque noir) ces grilles de surface.

tif. Dans la section suivante, nous verrons qu'une correspondance peut être établie entre ce système et le modèle Kondo en utilisant les degrés de liberté de 'charge' du circuit. Sous le processus de renormalisation, la conductance va converger, s'écouler 1 vers des points fixes Kondo non-triviaux à basse température. Dans la dernière section, nous nous concentrons sur la physique critique quantique et les comportements d'échelle qui apparaissent lorsqu'on introduit intentionnellement des perturbations dite 'pertinentes' au sens du groupe de renormalisation.

${ }^{1}$ De l'anglais to flow, terme utilisé dans le contexte de la renormalisation. 


\section{F.1 Contrôle de la quantification de la charge}

\section{Transistor "à un électron" proche de la limite de couplage fort}

La pièce métallique coloriée en violet dans la Fig. F.1 joue un rôle principal dans cette thèse. Elle sera appelée l'îlot, puisqu'elle est connectée au circuit par peu de canaux électroniques. La géométrie de l'échantillon règle l'énergie de charge $E_{C} \triangleq e^{2} /(2 C)$ (avec $e$, la charge élémentaire et $C$, la capacitance géométrique de l'îlot) typiquement requise pour ajouter/enlever un électron à l'îlot. Les effets "à un électron" sont donc importants aux température et énergies plus petites que cette échelle: $k_{B} T, e V \ll E_{C} \approx 25 \mu \mathrm{eV} \approx k_{B} \times 300 \mathrm{mK}$.

La connexion entre l'îlot et le circuit électrique (constitué de grands réservoirs électroniques) peut être ajusté par l'intermédiaire de QPCs réglables (en cyan). Dans cette section, nous considérons uniquement deux QPCs (voir Fig. F.2 a). Lorsque les transmissions de chacun des deux QPCs (de droite et de gauche) sont réglés pour être dans le régime tunnel $\tau_{L, R} \ll 1$, l'îlot n'est que faiblement connecté aux conducteurs environnants et sa charge est alors quantifiée en unité de $e$. Dans cette limite, le dispositif implémente le bien connu "transistor à un électron" (SET). Le nombre de charge sur l'îlot peut être ajusté en balayant la tension $V_{g}$ appliquée sur une grille latérale (par exemple, sur une grille de caractérisation, en orange sur Fig. F.1).

a

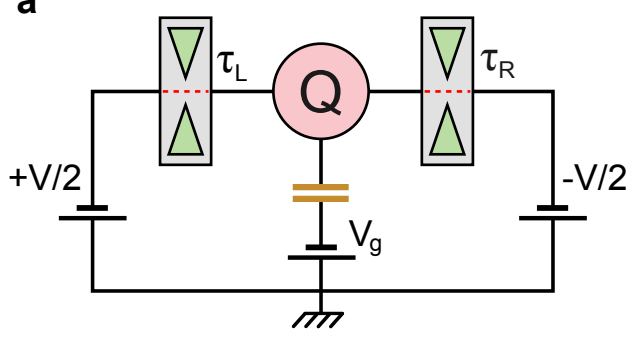

b $Q_{A}$

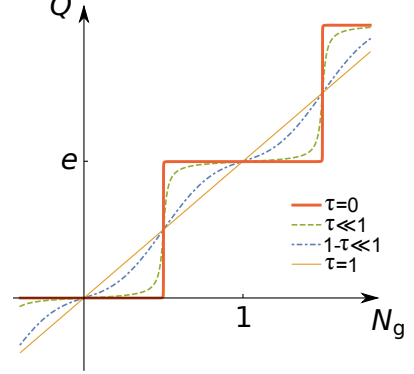

Figure F.2: Quantification de la charge dans un dispositif "à un électron". a, Schéma de l'échantillon. La conductance différentielle $G_{\text {SET }}$ est mesurée en appliquant une tension de biais $\mathrm{AC}$ au dispositif. En présence de quantification de la charge, cette conductance montre des oscillations de Coulomb en fonction de la tension $V_{g}$ appliquée sur un grille couplée capacitivement. bo, (adapté de [78]) La charge moyenne $Q$ est tracée à température nulle en fonction $N_{g} \triangleq V_{g} / \Delta V_{g}$ (où $\Delta V_{g}$ est la période des oscillations de Coulomb) pour différentes valeurs de connexion $\tau$ (la probabilité de transmission $\tau \triangleq \tau_{R}$, avec $\tau_{L}=0$ ). La quantification de la charge disparait complètement dès qu'un canal balistique connecte l'îlot $(\tau=1)$.

On peut alors observer des oscillations de Coulomb de la conductance du dispositif $G_{\mathrm{SET}}\left(V_{g}\right)$, car le courant ne peut circuler que lorsque deux états de 
charge successif de l'îlot sont dégénérés en énergie (les fluctuations thermiques autorisent une certaine plage autour de ces points de dégénérescence; ailleurs, la charge est gelée et le courant est bloqué). Le nombre d'électrons sur l'îlot est donc incrémenté d'une unité après chaque pic de conductance montré sur la Fig. F.3a. Dans cette figure, nous voyons que les oscillations disparaissent progressivement lorsque la transmission des canaux électroniques à travers un QPC tend vers la limite $\tau \longrightarrow 1$. Pour une étude plus systématique, nous traçons la visibilité des oscillations $\Delta Q \triangleq\left(G_{\mathrm{SET}}^{\max }-G_{\mathrm{SET}}^{\min }\right) /\left(G_{\mathrm{SET}}^{\mathrm{max}}+G_{\mathrm{SET}}^{\min }\right)$ en fonction de $\tau_{R}$. Toutes les courbes obtenues pour différentes valeurs fixées de $\tau_{L}$ tombent à zéro dès que la transmission devient balistique $\tau=1$, et aucune oscillation de Coulomb n'est observée au-delà de cette limite (voir Fig. F.3b).
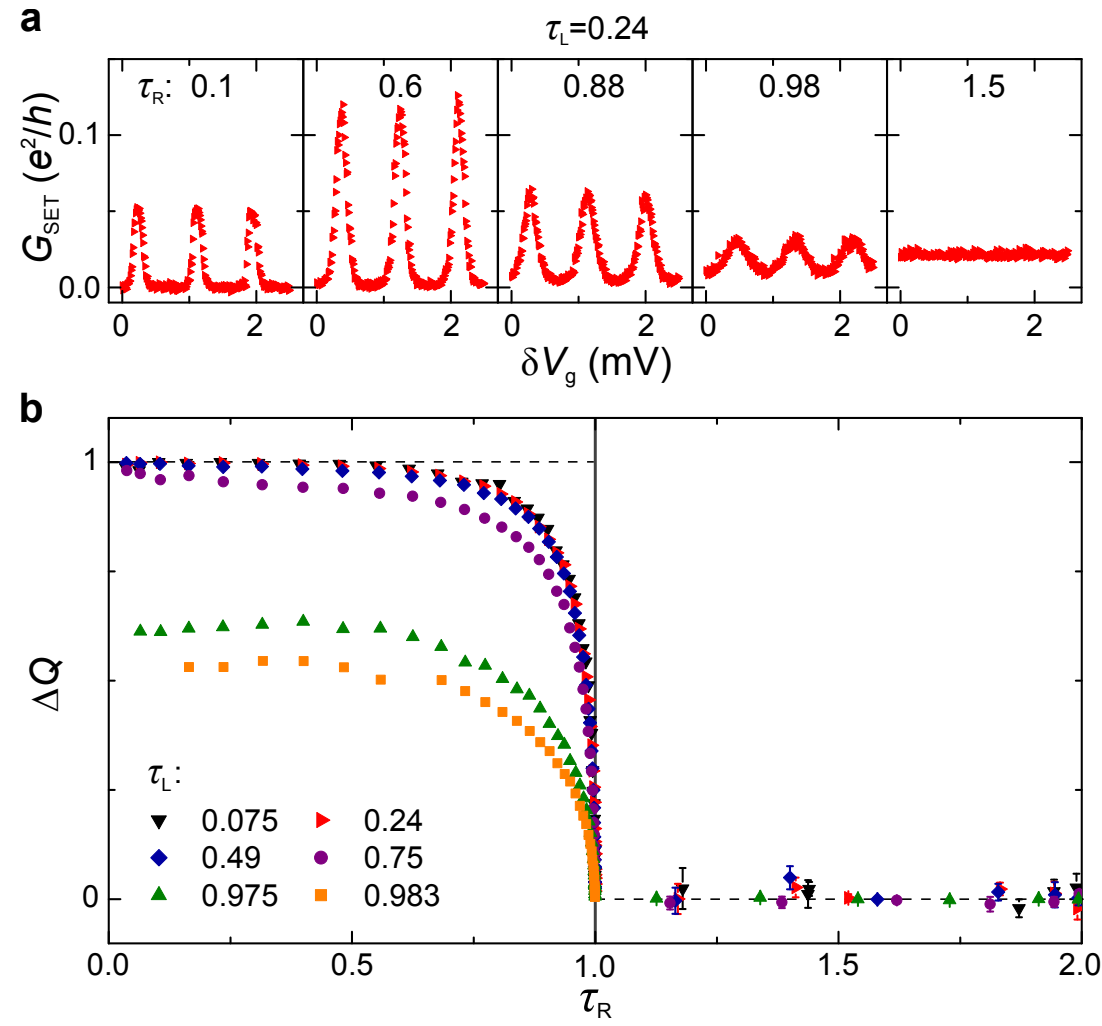

Figure F.3: Quantification de la charge en fonction de la connexion au circuit à $T \simeq 17 \mathbf{m K}$. a, Balayages de la conductance $G_{\mathrm{SET}}\left(\delta V_{\mathrm{g}}\right)$ pour des connexions ajustées à $\tau_{L}=0.24$, et $\tau_{R}=0.1,0.6,0.88,0.98$ et 1.5 , respectivement, de gauche à droite. $\mathbf{b}$, Visibilité des oscillations de $G_{\mathrm{SET}}$ en fonction de $\tau_{R}$. Chaque ensemble de points (différents symboles) correspond à point de fonctionnement de $\mathrm{QPC}_{L}$.

Il est connu, d'après la théorie, que les fluctuations quantiques de la charge brouillent la quantification de la charge (même à température nulle) [48]. En 1995, Matveev a montré que la quantification de la charge est complètement détruite dans 
la limite balistique, lorsqu'on considère une densité d'états continue dans l'îlot (voir Fig. F.2b) [78]. En 1993, une controverse est née à propos de ce critère de la destruction de la quantification de la charge puisque certaines expériences l'ont validé [119] alors que d'autres ont montrées des signatures de quantification audelà de la limite balistique [100]. Mais ces expériences pionnières étaient basées sur des îlots non-métalliques avec des densités d'états discrètes, et où la cohérence de phase des électrons peut donner lieu à de subtiles effets mésoscopiques [5]. La géométrie et les matériaux que nous avons utilisés pour fabriquer notre échantillon empêchent tout effet cohérent puisqu'un électron qui entre dans notre îlot y passe temps beaucoup plus long que son temps de cohérence de phase quantique.

\section{Comparaison quantitative à la théorie}

En supposant une densité d'états continue sur l'îlot et des électrons sans spin 2 , des prédictions quantitatives peuvent être établies pour ce système dans plusieurs cas limites. À basse température $k_{B} T \ll E_{C}$, nous comparons la visibilité $\Delta Q$ des oscillations de conductance au voisinage de la limite balistique $\tau_{R} \longrightarrow 1$ avec la théorie quantitative dans deux cas limites (couplage fort $1-\tau_{L} \ll 1$ [43] et asymétrique $\tau_{L} \ll 1[$ [80]). Nous sommes capables de caractériser tous les paramètres $\left(\tau_{L}, \tau_{R}, E_{C}\right.$ et $\left.T\right)$ de façon indépendante, et l'accord quantitatif obtenu dans la limite de couplage fort est donc établi sans aucun paramètre ajustable (voir Fig. F.4a).

Dans les deux limites, la théorie prédit une dépendance en $\sqrt{1-\tau_{R}}$ pour la visibilité des oscillations de Coulomb. Ce comportement en loi de puissance a aussi été observé pour les transmissions intermédiaires de $\tau_{L} \in[0,1]$. Aux hautes températures, cette dépendance devient totalement universelle sur toute la gamme $\tau_{R, L} \in[0,1]$ (comme le montre la convergence de tous les symboles correspondant à différents $\tau_{L}$ dans la Fig. F.4b). De plus, on note aussi sur cette figure que la quantification de la charge (mesurée par $\Delta Q$ ) est supprimée exponentiellement par la température $T$, comme prévu par la théorie en présence de fortes fluctuations thermiques $k_{B} T \gg E_{C} / \pi^{2}$ ([55] et références citées dedans).

\section{F.2 Observation de l'effet Kondo multi-canaux}

\section{Le modèle Kondo original et quelques variantes}

En 1964, Kondo a calculé la contribution à la résistivité de la diffusion des électrons de conduction par des impuretés magnétiques dans des alliages métalliques [62]. Cependant, son approche perturbative n'est pas valide aux basses températures

${ }^{2}$ La dégénérescence de spin est levée en pratique en appliquant un fort champ magnétique. 

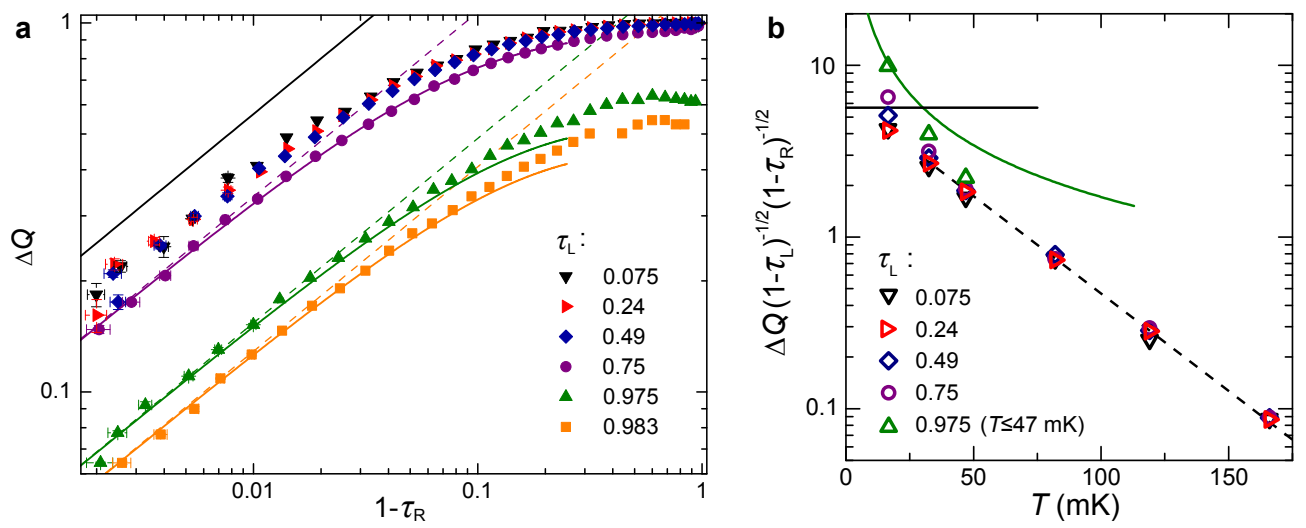

Figure F.4: Lois d'échelles de la quantification de la charge au voisinage du point balistique et suppression exponentielle avec la température. a, La visibilité $\Delta Q$ des oscillations de Coulomb à $T \approx 17 \mathrm{mK}$ est tracée en fonction de $1-\tau_{R}$ dans une échelle log-log, avec des ensembles de symboles différents pour chaque point de fonctionnement de $\mathrm{QPC}_{L}$. Les lignes continues sont les prédictions quantitative (sans paramètres ajustable) calculées en supposant $k_{B} T \ll$ $E_{C}, 1-\tau_{R} \ll 1$ et $\tau_{L} \ll 1$ (ligne droite toute en haut) ou $1-\tau_{L} \ll 1$ (trois lignes continues du bas). La loi de puissance $\Delta Q \propto \sqrt{1-\tau_{R}}$ (lignes droites tiretées) est observée systématiquement pour $1-\tau_{R} \lesssim 0.02$, même pour des $\tau_{L}$ intermédiaires. b, Les symboles en fonction de $T$, dans une échelle semi-log, montrent la visibilité réduite $\Delta Q / \sqrt{\left(1-\tau_{R}\right)\left(1-\tau_{L}\right)}$, extraite du régime où $1-\tau_{R}$ est suffisamment petit pour que $\Delta Q \propto \sqrt{1-\tau_{R}}$. Les lignes continues sont les prédictions quantitatives dans le régime quantique $k_{B} T \ll E_{C}$ montrées en a. La ligne droite tiretée correspond à une décroissance exponentielle proche de la prédiction théorique en présence de fortes fluctuations thermiques.

comparées ce qui appelé la température Kondo $T_{K}$. Ce problème à $\mathrm{N}$ corps est particulièrement bien adapté à la théorie de groupe de renormalisation. La première solution exacte a été trouvée par Wilson en utilisant le groupe de renormalisation numérique [131].

Le modèle de Kondo original est illustré en Fig. F.5. Il décrit une impureté magnétique (modélisée par un spin $\vec{S}$, tout au long de cette thèse, $S=1 / 2$ ) qui interagit avec une unique bande de conduction électronique (représentée par une réseau uni-dimensionnel) à travers un couplage anti-ferromagnétique $J$ localisé sur le site de l'impureté. À basse température, $J$ va prendre de nouvelles valeurs effectives, il est renormalisé vers $J \longrightarrow \infty$ [131]. Dans cette limite, l'impureté forme un singulet avec un électron, et cet écrantage parfait abouti à une description simple, en termes de liquide de Fermi [94]. Nozières et Blandin ont proposé une généralisation du modèle avec $N$ canaux de conductions indépendants [93], ce qui mène à un état fondamental qui n'est pas un liquide de Fermi à basse température 


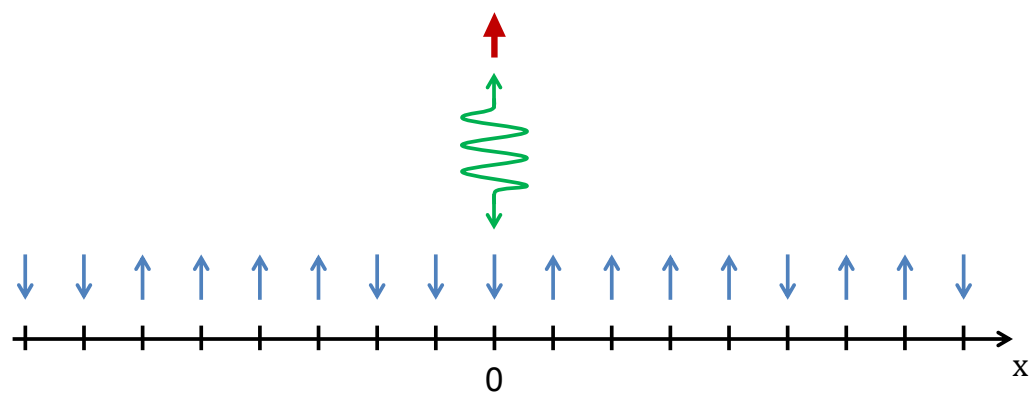

Figure F.5: Schéma expliquant le modèle Kondo. L'impureté $S=1 / 2$ est représentée par une flèche rouge. Les électrons de conductions (flèches blues) sont distribués sur un réseau. Pour simplifier, ce réseau est uni-dimensionnel. L'interaction anti-ferromagnétique est symbolisée par une flèche zigzag verte, sur le site de l'impureté.

dans le cas surécranté $N>2 S$. Selon la théorie du groupe de renormalisation, les couplages $J_{i}$ entre l'impureté quantique et chacun des canaux électronique sont renormalisés de façon effective lorsque la température baisse [10]. À température nulle, ils atteignent finalement un point fixe universel, qui ne dépend du nombre de canaux $N$ [93].

Dans notre dispositif, deux états de charge successifs peuvent être ajustés (en utilisant $V_{g}$ ) pour avoir la même énergie. Le nombre d'électrons sur l'îlot $n$ ou $n+1$ peut alors jouer le rôle d'un système quantique à deux niveaux, autrement dit, d'un pseudo-spin $S=1 / 2$. En 1991, Matveev a démontré, dans la limite de couplage faible, la correspondance exacte entre le modèle Kondo original (avec de vrais spins) et le blocage de Coulomb qui décrit notre système [79]. Cette correspondance, qui est valide au-delà de la limite tunnel [78, 72], met en jeu le couplage entre le pseudo-spin Kondo de 'charge' et un pseudo-spin de localisation des électrons (soit sur l'îlot, soit dehors).

\section{Observation d'effets Kondo multi-canaux et de comportements universels}

La conductance $G_{i}$ de chaque QPC va être renormalisée vers les points fixes Kondo de 'charge' à basse température. Dans la Fig. F.6, lorsqu'on ajuste les transmissions en jeu pour qu'elles soient égales ( $\tau \triangleq \tau_{1}=\tau_{3}$ et $\tau_{2}=0$ pour l'effet Kondo à deux canaux (2CK); $\tau \triangleq \tau_{1}=\tau_{2}=\tau_{3}$ pour trois canaux (3CK)) et la tension de grille au point de dégénérescence de charge $\left(\delta V_{g}=0\right)$, on observe que la conductance converge vers les points fixes Kondo prédits (qui est extrémal $G_{2 C K}^{*}=e^{2} / h$ dans le cas à deux canaux et intermédiaire $G_{3 C K}^{*}=2 \sin ^{2}(\pi / 5) e^{2} / h \approx 0.691 e^{2} / h$ dans 
le cas à trois canaux) [135]. Notez que ces points fixes sont universels, c'est-à-dire qu'ils ne dépendent d'aucun paramètre microscopique (transmissions $\tau$, énergie de charge $E_{C}$, etc.).
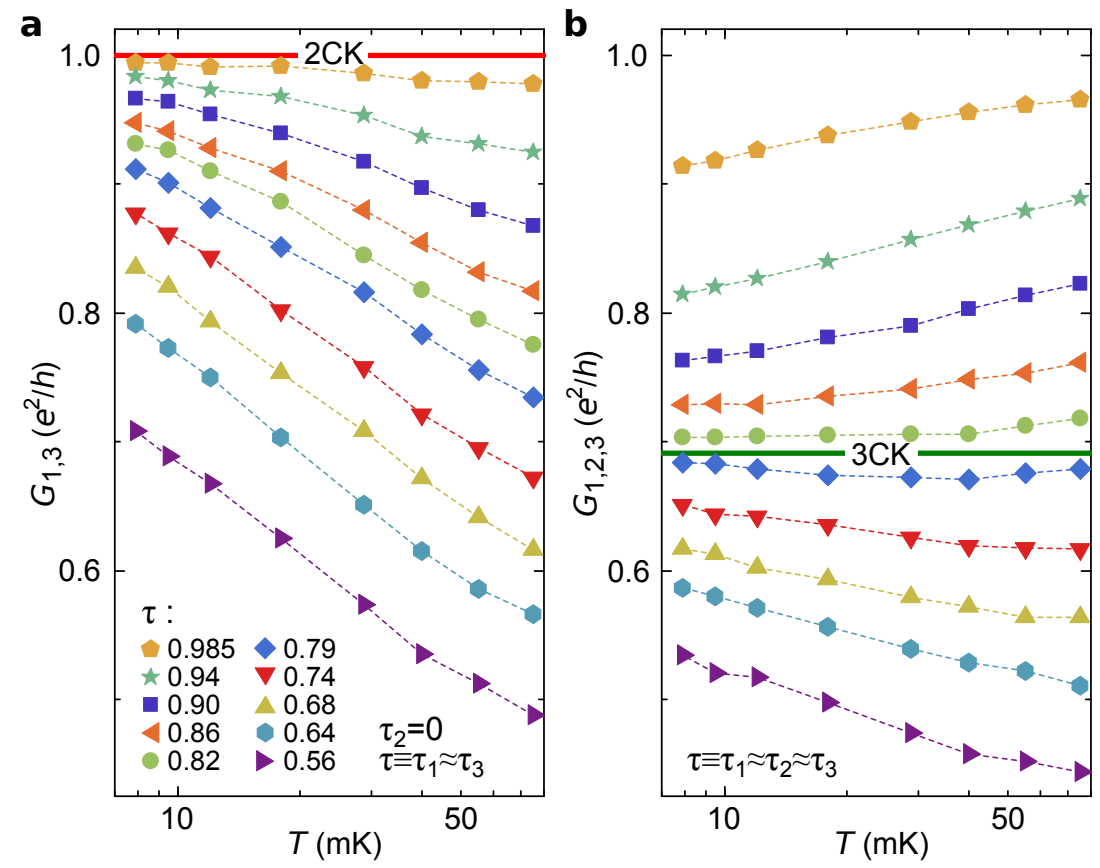

Figure F.6: Renormalisation vers les points fixes Kondo de 'charge' à deux et trois canaux. Pour le même jeu de transmissions $\tau$, les conductances individuelles au point de dégénérescence $\delta V_{g}=0$ sont tracées en fonction de la température $T \approx\{7.9,9.5,12,18,29,40,55,75\} \mathrm{mK}$ en échelle log. a, Lorsque la température baisse, la conductance converge vers le point fixe 2CK (ligne rouge) quelque soit la transmission dans le cas à deux canaux symétriques $\left(\tau_{1} \approx \tau_{3}\right)$. b, Avec trois canaux symétriques, elle converge vers $G_{3 \mathrm{CK}}^{*}$ (ligne verte).

Les propriétés d'échelle et d'universalité sont inhérentes au groupe de renormalisation [131]. Dans le modèle Kondo, l'évolution en température de n'importe quelle observable est une fonction universelle de la température réduite $T / T_{K}$, à condition que la température ait été suffisamment réduite pour que la renormalisation ait supprimé l'influence des perturbations non-pertinentes ${ }^{3}$ [92]. Mitchell et al. ont calculé la courbe universelle de conductance $G\left(T / T_{K}\right)$ complète (de $G\left(T / T_{K} \gg 1\right) \approx 0$ à $\left.G\left(T / T_{K} \ll 1\right) \approx G^{*}\right)$ pour les modèles Kondo de 'charge' à deux et trois canaux [85, 51]. Nous comparons nos mesures expérimentales à leurs calculs numériques exacts dans la Fig. F.7. Notre procédure consiste à remettre la

${ }^{3}$ Dans le jargon de la renormalisation, l'effet d'une perturbation non-pertinente (irrelevant en anglais) diminue à basse énergie. 
température à l'échelle en faisant correspondre le point de plus basse température avec la courbe théorique. On note qu'au moins les trois points de plus basse température sont dans le régime universel puisque leur évolution suit la courbe théorique. Cette procédure fonctionne sur plusieurs ordres de grandeurs en $T / T_{K}$, soulignant la grande plage sur laquelle notre dispositif est ajustable. Aux hautes températures, des effets non-universels apparaissent. En fait, cette transition vers un régime non-universel est complètement expliqué par les calculs du groupe de renormalisation numérique, en prenant en compte une énergie de charge finie. À partir de ce scaling en $T / T_{K}$, on peut extraire une relation entre la température Kondo $T_{K}$ et la force de couplage non-renormalisée $\tau$. Dans les encarts de la Fig. F.7, nous comparons $T_{K}$ à la théorie au voisinage des points fixes et dans le régime tunnel.
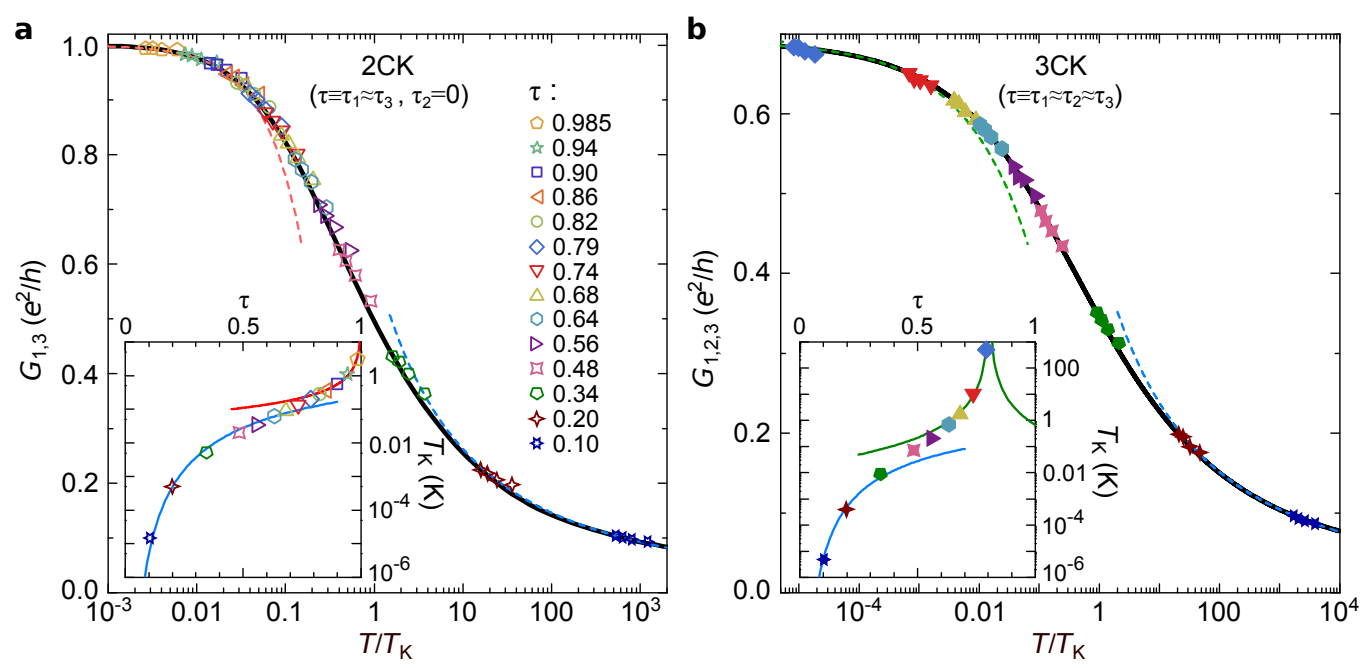

Figure F.7: Comparaison des mesures expérimentales aux courbes universelles de conductance du modèle Kondo de 'charge' à deux et trois canaux. Ici, les points expérimentaux sont tracés jusqu'à $T=29 \mathrm{mK}$ et quelques transmissions additionnelles sont montrées comparé à la Fig. F.6. Dans cette représentation semi-log, chaque ensemble de point à $\tau$ fixé est déplacé horizontalement de sorte que le point de plus basse température s'ajuste sur la courbe théorique (ligne noire continue). Cette procédure définie des températures d'échelle $T_{K}(\tau)$ qui sont tracées dans les encarts pour les configuration $2 \mathrm{CK}$ (a) et $3 \mathrm{CK}(\mathbf{b})$, et comparées aux prédictions théoriques (lignes colorés). Les lignes bleues correpondent au régime tunnel décrit par la théorie perturbative [10, 43]. Les lignes colorées tiretées sont les lois de puissance prédites pour la conductance au voisinage des points fixes Kondo [43, 51]. 


\section{F.3 Transition de phase quantique dans les système Kondo multi-canaux}

\section{Criticalité quantique}

Le travail sur les transitions de phase quantiques a été principalement motivé par l'un des plus important problème non-résolu en physique de la matière condensée, qui concerne le diagramme de phase compliqué de certains matériaux fortement corrélés. En particulier, la phase 'métal étrange' de laquelle émerge la supraconductivité des supraconducteurs à haute température critique a attiré beaucoup d'efforts théorique mais demeure encore mal comprise (voir [59] pour une revue récente et Fig. F.8 a). Cette phase montre des signatures de criticalité quantique comme une loi de puissance non-liquide de Fermi pour la résistivité en fonction de la température, ainsi qu'un élargissement de l'intervalle critique avec la température.
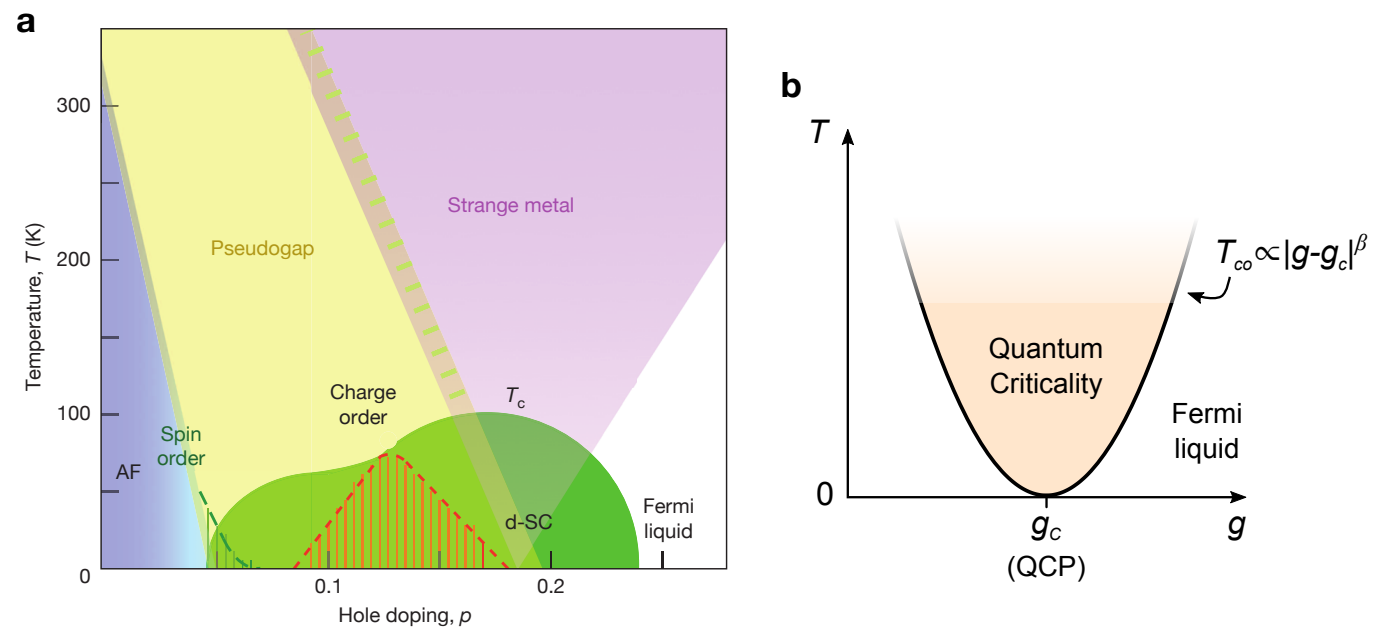

Figure F.8: Diagrammes de phase typiques. a, (reproduit et simplifié à partir de [59]) Diagramme de phase typique d'un oxyde de cuivre tracé en fonction du dopage en trous, ces matériaux peuvent présenter un ordre anti-ferromagnétique (AF, en bleu), un ordre de spin (hachures vertes), un ordre de charge (hachures rouges), un ordre supraconducteur $d$-wave (d-SC, en vert). Un liquide de Fermi est obtenu dans le régime à fort dopage (à basse température). b, Diagramme de phase typique montrant de la criticalité quantique lorsque le paramètre non-thermique $g$ est ajusté au voisinage du point critique quantique $(\mathrm{QCP})$ à basse température.

Contrairement aux phénomènes critiques classiques qui se déroulent à la température critique $T_{C}$ d'une transition de phase du second ordre, la criticalité quantique tire son origine des fluctuations quantiques qui existent même à température 
nulle, à un point critique quantique. Un système physique est amené à la criticalité quantique à l'aide d'un paramètre non-thermique $g$ (par exemple le dopage comme dans la Fig. F.8 a, la pression, ou une tension de grille). Il va présenter de la criticalité quantique sur un intervalle de paramètre qui commence du point critique $g=g_{c}$ à température nulle, et qui s'élargit selon une loi de puissance $T_{\text {co }} \propto\left(g-g_{c}\right)^{\gamma}$, où $\gamma$ est appelé 'exposant critique' et $T_{\text {co }}$ est la température de crossover en dessous de laquelle le système échappe à la criticalité quantique (voir Fig. F.8p) [107, 125].

Malgré les possibilités offertes par les nano-dispositifs ajustables pour comparer les mesures avec la théorie, les réalisations de criticalité quantique dans ce type de systèmes sont rares [81, 82, 60]. Des prédictions existent pour les points fixes Kondo sur-écrantés, qui sont connus pour le caractère non-trivial des exposants critiques de divers propriétés physiques ([3, 4] et références incluses). Dans notre implémentation de 'charge', nous pouvons observer un crossover de la criticalité quantique soit en introduisant une asymétrie dans la transmission $\tau$ des canaux, soit en levant la dégénérescence de charge $\delta V_{g}=0$.

\section{Développement d'une transition de phase quantique}

Dans la Fig. F.9, on règle $\delta V_{g}=0$, et on trace la conductance $G$ en fonction de la température. Chaque flèche pointe vers les basses températures et correspond à une configuration donnée $\left(\tau_{2} ; \tau_{1}=\tau_{3}\right)$. Ce graphe fournit donc une visualisation du flot de renormalisation de l'effet Kondo à trois canaux (avec deux canaux réglés symétriques $\tau_{1}=\tau_{3}$ ). Suivant le nombre de nombre de canaux symétriques qui partagent la meilleure connexion intrinsèque $\tau$ à l'îlot, les conductances individuelles $G$ vont converger vers le point fixe Kondo à un (disque bleu), deux (disque rouge) ou trois (disque vert) canaux.

Pour des transmissions symétriques $\tau_{1}=\tau_{2}=\tau_{3}$, le long de la diagonale, les conductances individuelles $G$ restent symétriques quelque soit la température. Cependant, ce réglage est assez critique puisqu'une asymétrie, aussi petite soitelle, augmente lorsque la température baisse. Ce graphe nous donne donc une vue directe sur le développement d'une transition de phase quantique.

Dans le régime de couplage fiable $\tau_{1,2,3} \ll 1$, où les canaux peuvent être traités indépendamment et avec une théorie perturbative [10], une augmentation des conductances est prédite [43]. La position du point fixe $J_{1 \mathrm{CK}}^{*} \longrightarrow \infty$ de l'effet Kondo de 'spin' à un canal correspond, de façon inattendue, à $G_{1 C K}^{*}=0$ pour notre dispositif. De ceci résulte les comportement non-monotone de la conductance et les nombreux croisements de flèches dans la partie supérieure du diagramme $\left(\tau_{2}>\tau_{1,3}\right)$.

Un remarquable dépassement du quantum de conductance $e^{2} / h$ par la conductance individuelle d'un unique canal est observé dans le flot vers le point fixe à 


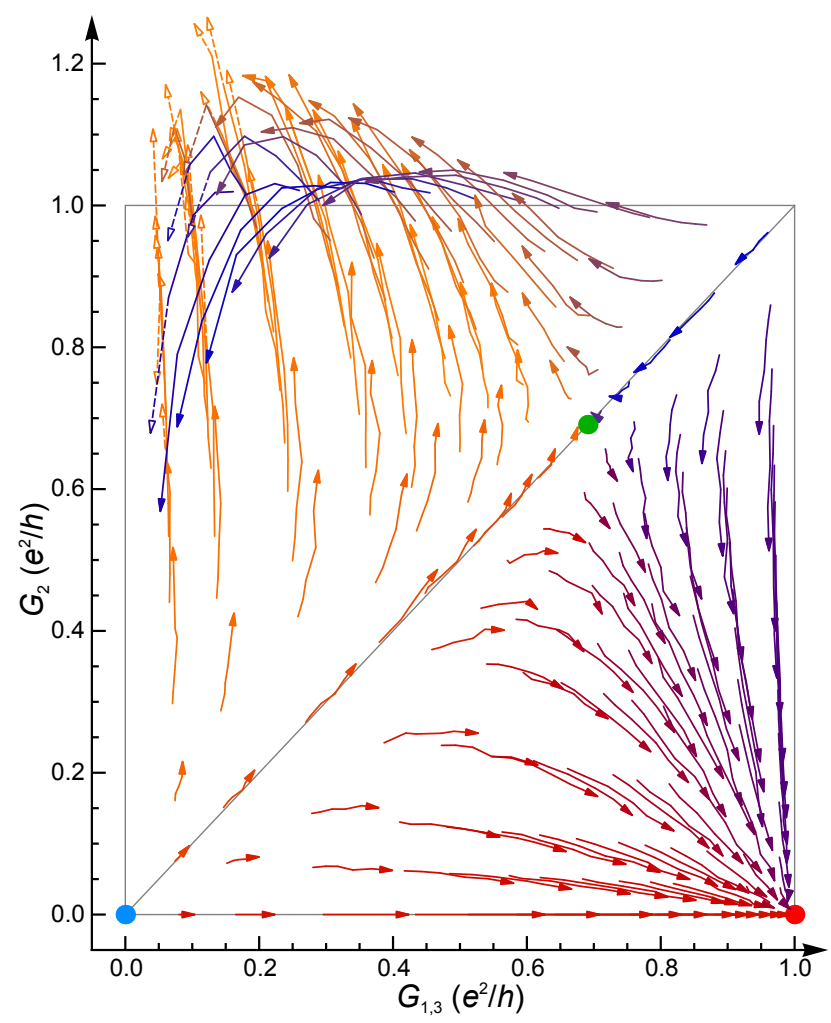

Figure F.9: Observation du flot de renormalisation 3CK. La moyenne des conductances $G_{1}$ et $G_{3}$ est tracée en fonction de $G_{2}$ au températures $T \approx\{7.9$, $9.5,12,18,29,40,55\} \mathrm{mK}$. La couleur des flèches correspond à leur orientation. L'incertitude des symboles ouverts et des lignes tiretées est inférieure à $0.1 e^{2} / h$ tandis qu'elle est inférieure à $0.05 e^{2} / h$ pour les symboles pleins et les lignes continues. Les points fixes Kondo sont indiqués par des disques colorés (1CK en cyan, $2 \mathrm{CK}$ en rouge et $3 \mathrm{CK}$ en vert).

un canal. Cette observation a été corroborée par les récents calculs de renormalisation numériques effectués par A.K. Mitchell. Un autre aspect essentiel de ce diagramme est la visualisation d'un crossover entre deux points fixes non-liquide de Fermi (du point fixe $3 \mathrm{CK}$ au point fixe $2 \mathrm{CK}$ ).

\section{Crossover de la criticalité quantique en utilisant un champ mag- nétique effectif}

Dans cette section, on règle les transmissions symétriques (soit $\tau \triangleq \tau_{1}=\tau_{3}$ et $\tau_{2}=0$, soit $\tau \triangleq \tau_{1}=\tau_{2}=\tau_{3}$ ). L'état critique quantique obtenu à basse température $T \ll T_{K}, E_{C}$ et son caractère distinct d'un liquide de Fermi sont fragiles. Ils sont détruits par une levée de la dégénérescence de charge. Un $\delta V_{g}$ non-nul favorise l'un 
des deux états du pseudo-spin de charge et détruit l'effet Kondo en agissant comme un champ magnétique effectif [79]. Ceci va mener le système vers un liquide de Fermi avec une échelle d'énergie typique donnée par la température de crossover $T_{\text {co }}$ (voir Fig. F.8p). Cette quantité est prédite pour dépendre de $\delta V_{g}$ selon une loi de puissance $T_{\text {co }} \propto \delta V_{g}^{\beta(N)}$ avec un exposant critique $\beta(N)=(2+N) / N$ [29].
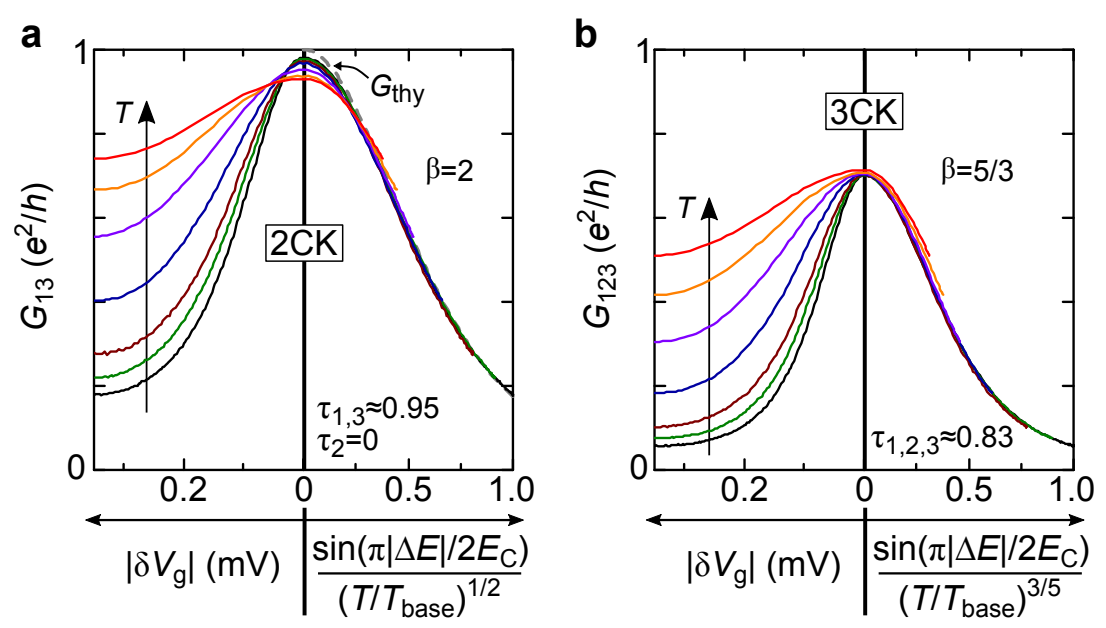

Figure F.10: Crossover de la criticalité quantique en levant la dégénérescence de charge. Un pic de conductance est montré à $T \approx\{7.9,9.5,12,18,29,40,55\} \mathrm{mK}$ pour une transmission $\tau$ sélectionnée au voisinage du point fixe 2CK (dans a) et $3 \mathrm{CK}$ (dans b). Les lignes continues sont les mesures expérimentales. Pour chaque graphe $\mathbf{a}$ et $\mathbf{b}$, deux échelles sont utilisées en abscisse: la tension de grille brute $\delta V_{g}$ (à gauche) et une remise à l'échelle $\sin \left(\pi \Delta E /\left(2 E_{C}\right)\right) /\left(T / T_{\text {base }}\right)^{1 / \beta}$, avec $T_{\text {base }}=7.9 \mathrm{mK}$ (à droite). La conductance adopte un comportement universel après la mise à l'échelle de l'abscisse (sauf pour les deux températures les plus élevées). La ligne tiretée grise $G_{\text {thy }}$ dans a montre la prédiction à température nulle pour $\tau=0.95$.

De plus, au voisinage du point critique quantique, $T_{\text {co }}$ est la seule échelle d'énergie à considérer, et la conductance doit être une fonction universelle de $T / T_{\text {co }}[102,85]$. L'expression quantitative de $T_{\mathrm{co}}(\Delta E)$ en fonction d'écart à la dégénérescence ${ }^{4} L$ 'expression de $\Delta E$ a été calculée par Fursaki et Matveev pour le modèle $2 \mathrm{CK}$ de 'charge', pour des $\Delta E$ arbitraires (au-delà de la loi de puissance $\Delta E \ll E_{C}$ ) [43]. Dans la Fig. 1.10a, nous utilisons cette expression pour mettre à l'échelle $\delta V_{g}$ et nous observons que les pics de conductances mesurés à différentes températures retombent tous sur une même courbe universelle. À partir de notre

\footnotetext{
${ }^{4}$ Comme expliqué plus haut, $\delta V_{g}$ agit comme un champ magnétique. On définit $\Delta E \triangleq$ $2 E_{C} \delta V_{g} / \Delta V_{g}$, où $E_{C} / k_{B} \approx 300 \mathrm{mK}$ est l'énergie de charge et $\Delta V_{g} \approx 0.70 \mathrm{mV}$ est la période des oscillations de Coulomb (ces deux valeurs numériques sont déterminées par la géométrie de l'échantillon, et elles sont indépendantes de $T$ ou $\tau$ ).
} 
connaissance de l'exposant critique $\beta(N)$, nous avons proposé une généralisation naive de ce scaling qui correspond bien à nos pics de conductance 3CK (voir Fig. 1.7p). Nous vérifions ainsi les lois de puissance universelles prédites pour la température de crossover $T_{\mathrm{co}}$ à petit $\delta V_{g}$ et nous démontrons un accord quantitatif avec la prédiction théorique complète de Furusaki et Matveev (ligne tiretée dans la Fig. F.10a) [43]. 


\section{Bibliography}

[1] David Aasen, Michael Hell, Ryan V. Mishmash, Andrew Higginbotham, Jeroen Danon, Martin Leijnse, Thomas S. Jespersen, Joshua A. Folk, Charles M. Marcus, Karsten Flensberg, and Jason Alicea. Milestones toward Majorana-based quantum computing. Physical Review X, 6:031016, Aug 2016.

[2] Ian Affleck, Andreas W. W. Ludwig, H.-B. Pang, and D. L. Cox. Relevance of anisotropy in the multichannel Kondo effect: Comparison of conformal field theory and numerical renormalization-group results. Physical Review $B, 45(14): 7918-7935,1992$.

[3] Ian Affleck and Andreas W.W. Ludwig. Critical theory of overscreened Kondo fixed points. Nuclear Physics B, 360(2):641 - 696, 1991.

[4] Ian Affleck and Andreas W.W. Ludwig. Exact conformal-field-theory results on the multichannel Kondo effect: Single-fermion Green's function, selfenergy, and resistivity. Physical Review B, 48(10):7297, 1993.

[5] Igor L. Aleiner and L.I. Glazman. Mesoscopic charge quantization. Physical Review B, 57(16):9608, 1998.

[6] C. Altimiras, U. Gennser, A. Cavanna, D. Mailly, and F. Pierre. Experimental test of the dynamical Coulomb blockade theory for short coherent conductors. Physical Review Letters, 99(25):256805, 2007.

[7] Carles Oriol Altimiras. Mécanismes inélastiques dans des circuits mésoscopiques réalisés dans des gaz bidimensionnels d'électrons. $\mathrm{PhD}$ thesis, Paris 11, 2010.

[8] S. Amasha, I.G. Rau, M. Grobis, R.M. Potok, H. Shtrikman, and D. Goldhaber-Gordon. Coulomb blockade in an open quantum dot. Physical Review Letters, 107(21):216804, 2011. 
[9] Philip W. Anderson, G. Yuval, and D.R. Hamann. Exact results in the Kondo problem. II. Scaling theory, qualitatively correct solution, and some new results on one-dimensional classical statistical models. Physical Review B, 1(11):4464, 1970.

[10] P.W. Anderson. A poor man's derivation of scaling laws for the Kondo problem. Journal of Physics C: Solid State Physics, 3(12):2436, 1970.

[11] Tsuneya Ando, Alan B. Fowler, and Frank Stern. Electronic properties of two-dimensional systems. Reviews of Modern Physics, 54(2):437, 1982.

[12] N. Andrei. Diagonalization of the Kondo Hamiltonian. Physical Review Letters, 45(5):379-382, 1980.

[13] N. Andrei and C. Destri. Solution of the multichannel Kondo problem. Physical Review Letters, 52(5):364, 1984.

[14] Michael Arnold, Tobias Langenbruch, and Johann Kroha. Stable twochannel Kondo fixed point of an SU(3) quantum defect in a metal: Renormalization-group analysis and conductance spikes. Physical Review Letters, 99(18):186601, 2007.

[15] J.G. Bednorz and K.A. Müller. Possible high-Tc superconductivity in the Ba-La-Cu-O system. Zeitschrift für Physik B Condensed Matter, 64(2):189_ 193, 1986.

[16] Ya.M. Blanter and Markus Büttiker. Shot noise in mesoscopic conductors. Physics reports, 336(1):1-166, 2000.

[17] Immanuel Bloch, Jean Dalibard, and Sylvain Nascimbène. Quantum simulations with ultracold quantum gases. Nature Physics, 8(4):267-276, 2012.

[18] László Borda, Lars Fritz, Natan Andrei, and Gergely Zaránd. Theory of inelastic scattering from quantum impurities. Physical Review B, 75(23):235112, 2007.

[19] David Ian Bradley, Richard Edwin George, David Gunnarsson, Richard Peter Haley, Hannele Heikkinen, Yu.A. Pashkin, J. Penttilä, Jonathan Robert Prance, Mika Prunnila, Leif Roschier, and M. Sarsby. Nanoelectronic primary thermometry below 4 mk. Nature communications, 7, 2016.

[20] Ralf Bulla, Theo A. Costi, and Thomas Pruschke. Numerical renormalization group method for quantum impurity systems. Reviews of Modern Physics, 80(2):395, 2008. 
[21] M. Büttiker. Four-terminal phase-coherent conductance. Physical Review Letters, 57(14):1761, 1986.

[22] M. Büttiker. Quantized transmission of a saddle-point constriction. Physical Review B, 41(11):7906, 1990.

[23] M. Büttiker, Y. Imry, R. Landauer, and S. Pinhas. Generalized many-channel conductance formula with application to small rings. Physical Review B, 31(10):6207, 1985.

[24] John Cardy. Scaling and renormalization in statistical physics, volume 5. Cambridge university press, 1996.

[25] D.B. Chklovskii, B.I. Shklovskii, and L.I. Glazman. Electrostatics of edge channels. Physical Review B, 46(7):4026, 1992.

[26] P. Coleman, L.B. Ioffe, and A.M. Tsvelik. Simple formulation of the twochannel Kondo model. Physical Review B, 52(9):6611, 1995.

[27] P. Coleman and A.J. Schofield. Simple description of the anisotropic twochannel Kondo problem. Physical Review Letters, 75(11):2184, 1995.

[28] Piers Coleman and Andrew J. Schofield. Quantum criticality. Nature, 433(7023):226-229, 2005.

[29] D. L. Cox and A. Zawadowski. Exotic Kondo effects in metals: Magnetic ions in a crystalline electric field and tunnelling centres. Advances in Physics, 47(5):599-942, 1998.

[30] D.M. Cragg, P. Lloyd, and Ph. Nozières. On the ground states of some sd exchange Kondo Hamiltonians. Journal of Physics C: Solid State Physics, 13(5):803, 1980.

[31] R. Cron, E. Vecino, Devoret M., Esteve, D., P. Joyez, A. L. Yeyati, and C. Urbina. Dynamical Coulomb blockade in quantum point contacts. In T. Martin, G. Montambaux, and J. Trân Thanh Vân, editors, Electronic Correlations: from Meso- to Nanophysics, page 17, 2001.

[32] Ronald Cron. Les contacts atomiques: un banc d'essai pour la physique mésoscopique. PhD thesis, Université Pierre et Marie Curie-Paris VI, 2001.

[33] Sara M. Cronenwett, Tjerk H. Oosterkamp, and Leo P. Kouwenhoven. A tunable Kondo effect in quantum dots. Science, 281(5376):540-544, 1998. 
[34] S.M. Cronenwett, S.M. Maurer, S.R. Patel, C.M. Marcus, C.I. Duruöz, and J.S. Harris Jr. Mesoscopic Coulomb blockade in one-channel quantum dots. Physical Review Letters, 81(26):5904, 1998.

[35] C.H. Crouch, C. Livermore, R.M. Westervelt, K.L. Campman, and A.C. Gossard. Coulomb oscillations in partially open quantum dots. Superlattices and microstructures, 20(3):377-381, 1996.

[36] J. Custers, P. Gegenwart, H. Wilhelm, K. Neumaier, Y. Tokiwa, O. Trovarelli, C. Geibel, F. Steglich, C. Pépin, and P. Coleman. The break-up of heavy electrons at a quantum critical point. Nature, 424(6948):524-527, 2003.

[37] Michel H. Devoret and Hermann Grabert. Single charge tunneling: Coulomb blockade phenomena in nanostructures:[Proceedings of the NATO Advanced Study Institute on Single charge tunneling, held March 5-15, 1991, in Les Houches, France]. Plenum Press, 1992.

[38] David S. Duncan, Carol Livermore, Robert M. Westervelt, Kevin D. Maranowski, and Arthur C. Gossard. Direct measurement of the destruction of charge quantization in a single-electron box. Applied Physics Letters, 74(7):1045-1047, 1999.

[39] V.J. Emery and S. Kivelson. Mapping of the two-channel Kondo problem to a resonant-level model. Physical Review B, 46(17):10812, 1992.

[40] M. Fabrizio, Alexander O. Gogolin, and Ph. Nozières. Anderson-Yuval approach to the multichannel Kondo problem. Physical Review B, 51(22):16088, 1995.

[41] Stefan Frank, Philippe Poncharal, Z.L. Wang, and Walt A. de Heer. Carbon nanotube quantum resistors. Science, 280(5370):1744-1746, 1998.

[42] Theodore A. Fulton and Gerald J. Dolan. Observation of single-electron charging effects in small tunnel junctions. Physical Review Letters, 59(1):109, 1987.

[43] A. Furusaki and K. A. Matveev. Theory of strong inelastic cotunneling. Physical Review B, 52(23):16676-16695, 1995.

[44] Philipp Gegenwart, Qimiao Si, and Frank Steglich. Quantum criticality in heavy-fermion metals. Nature Physics, 4(3):186-197, 2008. 
[45] Antoine Georges, Gabriel Kotliar, Werner Krauth, and Marcelo J. Rozenberg. Dynamical mean-field theory of strongly correlated fermion systems and the limit of infinite dimensions. Reviews of Modern Physics, 68(1):13, 1996.

[46] Thierry Giamarchi. Quantum physics in one dimension. Clarendon Oxford, 2004.

[47] Steven M. Girvin. The quantum Hall effect: novel excitations and broken symmetries. In Aspects topologiques de la physique en basse dimension. Topological aspects of low dimensional systems, pages 53-175. Springer, 1999.

[48] L.I. Glazman and K.A. Matveev. Lifting of the Coulomb blockade of one-electron tunneling by quantum fluctuations. Soviet physics, JETP, 71:1031-1037, 1990.

[49] David Goldhaber-Gordon, Hadas Shtrikman, D. Mahalu, David AbuschMagder, U. Meirav, and M.A. Kastner. Kondo effect in a single-electron transistor. Nature, 391(6663):156-159, 1998.

[50] Andrew A. Houck, Hakan E. Türeci, and Jens Koch. On-chip quantum simulation with superconducting circuits. Nature Physics, 8(4):292-299, 2012.

[51] Z. Iftikhar, A. Anthore, F. D. Parmentier, U. Gennser, A. Ouerghi, A. Cavanna, A.K. Mitchell, C. Mora, P. Simon, and F. Pierre. Observation of the three-channel Kondo effect. Unpublished work, in preparation.

[52] Z. Iftikhar, S. Jezouin, A. Anthore, U. Gennser, F. D. Parmentier, A. Cavanna, and F. Pierre. Two-channel Kondo effect and renormalization flow with macroscopic quantum charge states. Nature, 526:233-236, 2015.

[53] Gert-Ludwig Ingold and Yu.V. Nazarov. Charge tunneling rates in ultrasmall junctions. In Single charge tunneling, pages 21-107. Springer, 1992.

[54] C. Jayaprakash, H.R. Krishna-Murthy, and J.W. Wilkins. Two-impurity Kondo problem. Physical Review Letters, 47(10):737, 1981.

[55] S. Jezouin, Z. Iftikhar, A. Anthore, F.D. Parmentier, U. Gennser, A. Cavanna, A. Ouerghi, I.P. Levkivskyi, E. Idrisov, E.V. Sukhorukov, L.I. Glazman, and F. Pierre. Controlling charge quantization with quantum fluctuations. Nature, 536(7614):58-62, 2016. 
[56] P. Joyez, V. Bouchiat, D. Esteve, C. Urbina, and M.H. Devoret. Strong tunneling in the single-electron transistor. Physical Review Letters, 79(7):1349, 1997.

[57] P. Joyez and D. Esteve. Single-electron tunneling at high temperature. Physical Review B, 56(4):1848, 1997.

[58] L.P. Kadanoff. Critical Phenomena, Proceedings of the Enrico Fermi Summer School Course. Academic, New York, 1971.

[59] B. Keimer, S.A. Kivelson, M.R. Norman, S. Uchida, and J. Zaanen. From quantum matter to high-temperature superconductivity in copper oxides. Nature, 518(7538):179-186, 2015.

[60] A. J. Keller, L. Peeters, C. P. Moca, I. Weymann, D. Mahalu, V. Umansky, G. Zaránd, and D. Goldhaber-Gordon. Universal Fermi liquid crossover and quantum criticality in a mesoscopic system. Nature, 526:237-240, 2015.

[61] A.Yu. Kitaev. Unpaired majorana fermions in quantum wires. PhysicsUspekhi, 44(10S):131, 2001.

[62] Jun Kondo. Resistance minimum in dilute magnetic alloys. Progress of Theoretical Physics, 32(1):37-49, 1964.

[63] S.E. Korshunov. Coherent and incoherent tunneling in a Josephson junction with a "periodic" dissipation. Soviet Journal of Experimental and Theoretical Physics Letters, 45:434, 1987.

[64] Leo Kouwenhoven and Leonid Glazman. Revival of the Kondo effect. Physics world, 14(1):33, 2001.

[65] L.P. Kouwenhoven, C.M. Marcus, P.L. McEuen, S. Tarucha, R.M. Westervelt, and N.S. Wingreen. Electron transport in quantum dots. In Kluwer Series, editor, Mesoscopic Electron Transport, volume E345, pages 105214, 1997.

[66] L.P. Kouwenhoven, N.C. van der Vaart, A.T. Johnson, W. Kool, C.J.P.M. Harmans, J.G. Williamson, A.A.M. Staring, and C.T. Foxon. Single electron charging effects in semiconductor quantum dots. Zeitschrift für Physik $B$ Condensed Matter, 85(3):367-373, 1991.

[67] Rolf Landauer. Spatial variation of currents and fields due to localized scatterers in metallic conduction. IBM Journal of Research and Development, 1(3):223-231, 1957. 
[68] Robert B Laughlin. Nobel lecture: fractional quantization. Reviews of Modern Physics, 71(4):863, 1999.

[69] Robert B. Laughlin and David Pines. The theory of everything. Proceedings of the National Academy of Sciences, 97(1):28-31, 2000.

[70] Karyn Le Hur. Entanglement entropy, decoherence, and quantum phase transitions of a dissipative two-level system. Annals of Physics, 323(9):2208-2240, 2008.

[71] Karyn Le Hur and Georg Seelig. Capacitance of a quantum dot from the channel-anisotropic two-channel Kondo model. Physical Review B, 65(16):165338, 2002.

[72] Eran Lebanon, Avraham Schiller, and Frithjof B. Anders. Coulomb blockade in quantum boxes. Physical Review B, 68(4), 2003.

[73] I.P. Levkivskyi, E. Idrisov, and E.V. Sukhorukov. Untitled. in preparation.

[74] Jiutao Li, Wolf-Dieter Schneider, Richard Berndt, and Bernard Delley. Kondo scattering observed at a single magnetic impurity. Physical Review Letters, 80(13):2893, 1998.

[75] H.v. Löhneysen, T. Pietrus, G. Portisch, H.G. Schlager, A. Schröder, M. Sieck, and T. Trappmann. Non-Fermi-liquid behavior in a heavy-fermion alloy at a magnetic instability. Physical Review Letters, 72(20):3262, 1994.

[76] V. Madhavan, W. Chen, T. Jamneala, M.F. Crommie, and N.S. Wingreen. Tunneling into a single magnetic atom: spectroscopic evidence of the kondo resonance. Science, 280(5363):567-569, 1998.

[77] S. Martin, A.T. Fiory, R.M. Fleming, L.F. Schneemeyer, and J.V. Waszczak. Normal-state transport properties of $\mathrm{Bi}_{2+x} \mathrm{Sr}_{2-y} \mathrm{CuO}_{6+\delta}$ crystals. Physical Review B, 41(1):846, 1990.

[78] K. A. Matveev. Coulomb blockade at almost perfect transmission. Physical Review B, 51(3):1743-1751, 1995.

[79] K.A. Matveev. Quantum fluctuations of the charge of a metal particle under the Coulomb blockade conditions. Soviet physics, JETP, 72(5):892-899, 1991.

[80] K.A. Matveev and A.V. Andreev. Thermopower of a single-electron transistor in the regime of strong inelastic cotunneling. Physical Review B, 66(4):045301, 2002. 
[81] Henok T. Mebrahtu, Ivan V. Borzenets, Dong E. Liu, Huaixiu Zheng, Yuriy V. Bomze, Alex I. Smirnov, Harold U. Baranger, and Gleb Finkelstein. Quantum phase transition in a resonant level coupled to interacting leads. Nature, 488(7409):61-64, 2012.

[82] H.T. Mebrahtu, I.V. Borzenets, H. Zheng, Yu.V. Bomze, A.I. Smirnov, Serge Florens, H.U. Baranger, and G. Finkelstein. Observation of Majorana quantum critical behaviour in a resonant level coupled to a dissipative environment. Nature Physics, 9(11):732-737, 2013.

[83] Pankaj Mehta, Natan Andrei, P. Coleman, L. Borda, and Gergely Zarand. Regular and singular Fermi-liquid fixed points in quantum impurity models. Physical Review B, 72(1):014430, 2005.

[84] Robert Andrews Millikan. On the elementary electrical charge and the Avogadro constant. Physical Review, 2(2):109, 1913.

[85] A.K. Mitchell, L.A. Landau, L. Fritz, and E. Sela. Universality and scaling in a charge two-channel Kondo device. Physical Review Letters, 116(15), 2016.

[86] Andrew K. Mitchell, Martin R. Galpin, Samuel Wilson-Fletcher, David E. Logan, and Ralf Bulla. Generalized Wilson chain for solving multichannel quantum impurity problems. Physical Review B, 89(12):121105, 2014.

[87] Andrew K. Mitchell and Eran Sela. Universal low-temperature crossover in two-channel Kondo models. Physical Review B, 85(23):235127, 2012.

[88] Christophe Mora and Karyn Le Hur. Probing dynamics of Majorana fermions in quantum impurity systems. Physical Review B, 88(24):241302, 2013.

[89] Chetan Nayak, Steven H. Simon, Ady Stern, Michael Freedman, and Sankar Das Sarma. Non-Abelian anyons and topological quantum computation. Reviews of Modern Physics, 80(3):1083, 2008.

[90] Yuli V. Nazarov. Coulomb blockade without tunnel junctions. Physical Review Letters, 82(6):1245, 1999.

[91] Yuli V. Nazarov and Yaroslav M. Blanter. Quantum transport: introduction to nanoscience. Cambridge University Press, 2009.

[92] Ph. Nozières. Kondo effect for spin 1/2 impurity a minimal effort scaling approach. Journal de Physique, 39(10):8, 1978. 
[93] Ph. Nozières and A. Blandin. Kondo effect in real metals. Journal de Physique, 41(3):19, 1980.

[94] Philippe Nozières. A "Fermi-liquid" description of the Kondo problem at low temperatures. Journal of low temperature physics, 17(1-2):31-42, 1974.

[95] A.A. Odintsov, G. Falci, and Gerd Schön. Single-electron tunneling in systems of small junctions coupled to an electromagnetic environment. Physical Review B, 44(23):13089, 1991.

[96] Yuval Oreg and David Goldhaber-Gordon. Two-channel Kondo effect in a modified single electron transistor. Physical Review Letters, 90(13), 2003.

[97] Kiryl Pakrouski, Michael R. Peterson, Thierry Jolicoeur, Vito W. Scarola, Chetan Nayak, and Matthias Troyer. Phase diagram of the $v=5 / 2$ fractional quantum hall effect: effects of landau-level mixing and nonzero width effect: effects of Landau-level mixing and nonzero width. Physical Review $X, 5(2): 021004,2015$.

[98] J.J. Parks, A.R. Champagne, T.A. Costi, W.W. Shum, A.N. Pasupathy, E. Neuscamman, S. Flores-Torres, P.S. Cornaglia, A.A. Aligia, C.A. Balseiro, G. K.-L. Chan, H. D. Abruna, and Ralph D. C. Mechanical control of spin states in spin-1 molecules and the underscreened Kondo effect. Science, 328(5984):1370-1373, 2010.

[99] F.D. Parmentier, A. Anthore, S. Jezouin, H. Le Sueur, U. Gennser, A. Cavanna, D. Mailly, and F. Pierre. Strong back-action of a linear circuit on a single electronic quantum channel. Nature Physics, 7(12):935-938, 2011.

[100] C. Pasquier, U. Meirav, F.I.B. Williams, D.C. Glattli, Y. Jin, and B. Etienne. Quantum limitation on Coulomb blockade observed in a 2D electron system. Physical Review Letters, 70(1):69, 1993.

[101] R.M. Potok, I.G. Rau, Hadas Shtrikman, Yuval Oreg, and D. GoldhaberGordon. Observation of the two-channel Kondo effect. Nature, 446(7132):167-171, 2007.

[102] M. Pustilnik, L. Borda, L.I. Glazman, and J. von Delft. Quantum phase transition in a two-channel-Kondo quantum dot device. Physical Review B, 69(11):115316, 2004.

[103] D.C. Ralph, A.W.W. Ludwig, Jan von Delft, and R.A. Buhrman. 2-channel Kondo scaling in conductance signals from 2 level tunneling systems. Physical Review Letters, 72(7):1064, 1994. 
[104] D.C. Ralph, A.W.W. Ludwig, Jan von Delft, and R.A. Buhrman. Ralph et al. reply. Physical Review Letters, 75(4):770-770, 1995.

[105] Nicholas Read and Dmitry Green. Paired states of fermions in two dimensions with breaking of parity and time-reversal symmetries and the fractional quantum Hall effect. Physical Review B, 61(15):10267, 2000.

[106] Nicolas Roch, Serge Florens, Theo A. Costi, Wolfgang Wernsdorfer, and Franck Balestro. Observation of the underscreened Kondo effect in a molecular transistor. Physical Review Letters, 103(19):197202, 2009.

[107] Subir Sachdev and Bernhard Keimer. Quantum criticality. arXiv preprint arXiv:1102.4628, 2011.

[108] L. Saminadayar, D.C. Glattli, Y. Jin, and B. Etienne. Observation of the e/3 fractionally charged Laughlin quasiparticle. Physical Review Letters, 79(13):2526, 1997.

[109] Elke Scheer, Philippe Joyez, Daniel Esteve, Cristián Urbina, and Michel H. Devoret. Conduction channel transmissions of atomic-size aluminum contacts. Physical Review Letters, 78(18):3535, 1997.

[110] Gerd Schön and Andrej Dmitievič Zaikin. Quantum coherent effects, phase transitions, and the dissipative dynamics of ultra small tunnel junctions. Physics Reports, 198(5-6):237-412, 1990.

[111] Anirvan M. Sengupta and Antoine Georges. Emery-Kivelson solution of the two-channel Kondo problem. Physical Review B, 49(14):10020, 1994.

[112] P. Simon and C. Mora. Scaling analysis near the non-Fermi liquid multichannel kondo fixed. Unpublished work.

[113] K.M. Stadler, A.K. Mitchell, J. von Delft, and A. Weichselbaum. Interleaved numerical renormalization group as an efficient multiband impurity solver. Physical Review B, 93(23):235101, 2016.

[114] Horst L. Stormer. Nobel lecture: the fractional quantum Hall effect. Reviews of Modern Physics, 71(4):875, 1999.

[115] G. Toulouse. Exact expression of energy of Kondo Hamiltonian base state for a particular $j_{z}$-value. Comptes rendus hebdomadaires des séances de l'Académie des Sciences Serie B, 268(18):1200, 1969. 
[116] A.M. Tsvelick. The transport properties of magnetic alloys with multichannel Kondo impurities. Journal of Physics: Condensed Matter, 2(12):2833, 1990.

[117] A.M. Tsvelick and P.B. Wiegmann. Solution of the n-channel Kondo problem (scaling and integrability). Zeitschrift für Physik B Condensed Matter, 54(3):201-206, 1984.

[118] G.J. van den Berg and J. de Nobel. Les propriétés à basses températures des alliages des métaux «normaux» avec des solutés de transition. Journal de Physique et le Radium, 23(10):665-671, 1962.

[119] N.C. van der Vaart, A.T. Johnson, L.P. Kouwenhoven, D.J. Maas, W. de Jong, M.P. de Ruyter van Steveninck, A. van der Enden, C.J.P.M. Harmans, and C.T. Foxon. Charging effects in quantum dots at high magnetic fields. Physica B: Condensed Matter, 189(1-4):99-110, 1993.

[120] W. G. van der Wiel. Electron transport and coherence in semiconductor quantum dots and rings. PhD thesis, Technische Universiteit Delft, 2002.

[121] W. G. van der Wiel, S. De Franceschi, T. Fujisawa, J. M. Elzerman, S. Tarucha, and L. P. Kouwenhoven. The Kondo effect in the unitary limit. Science, 289(5487):2105-2108, 2000.

[122] B.J. van Wees, H. van Houten, C.W.J. Beenakker, J.Gr. Williamson, L.P. Kouwenhoven, D. van der Marel, and C.T. Foxon. Quantized conductance of point contacts in a two-dimensional electron gas. Physical Review Letters, 60(9):848, 1988.

[123] P.B. Vigman. Exact solution of sd exchange model at $\mathrm{t}=0$. JETP Letters, $31: 364,1980$.

[124] K. Vladár and A. Zawadowski. Theory of the interaction between electrons and the two-level system in amorphous metals. I. Noncommutative model Hamiltonian and scaling of first order. Physical Review B, 28(3):1564-1581, 1983.

[125] Matthias Vojta. Quantum phase transitions. Reports on Progress in Physics, 66(12):2069, 2003.

[126] Matthias Vojta. Impurity quantum phase transitions. Philosophical Magazine, 86(13-14):1807-1846, 2006. 
[127] K. von Klitzing, Gerhard Dorda, and Michael Pepper. New method for highaccuracy determination of the fine-structure constant based on quantized hall resistance. Physical Review Letters, 45(6):494, 1980.

[128] Richard A. Webb, Sean Washburn, C.P. Umbach, and R.B. Laibowitz. Observation of $h / e$ Aharonov-Bohm oscillations in normal-metal rings. Physical Review Letters, 54(25):2696, 1985.

[129] D.A. Wharam, T.J. Thornton, R. Newbury, M. Pepper, H. Ahmed, J.E.F. Frost, D.G. Hasko, D.C. Peacock, D.A. Ritchie, and G.A.C. Jones. Onedimensional transport and the quantisation of the ballistic resistance. Journal of Physics C: Solid State Physics, 21(8):L209, 1988.

[130] Frank Wilczek. Majorana returns. Nature Physics, 5(9):614-618, 2009.

[131] Kenneth G. Wilson. The renormalization group: Critical phenomena and the Kondo problem. Reviews of Modern Physics, 47(4):773-840, 1975.

[132] Kenneth G. Wilson and John Kogut. The renormalization group and the $\varepsilon$ expansion. Physics Reports, 12(2):75-199, 1974.

[133] Ned S. Wingreen, Boris L. Altshuler, and Yigal Meir. Comment on "2channel Kondo scaling in conductance signals from 2-level tunneling systems". Physical Review Letters, 75(4):769-769, 1995.

[134] Hangmo Yi and C.L. Kane. Coulomb blockade in a quantum dot coupled strongly to a lead. Physical Review B, 53(19):12956, 1996.

[135] Hangmo Yi and C.L. Kane. Quantum Brownian motion in a periodic potential and the multichannel Kondo problem. Physical Review B, 57(10):R5579-R5582, 1998.

[136] Gergely Zaránd, Gergely T. Zimányi, and Frank Wilhelm. Two-channel versus infinite-channel Kondo models for the single-electron transistor. Physical Review B, 62(12):8137, 2000.

[137] Katrin Zimmermann, Anna Jordan, Frédéric Gay, Kenji Watanabe, Takashi Taniguchi, Zheng Han, Vincent Bouchiat, Hermann Sellier, and Benjamin Sacépé. Gate-tunable transmission of quantum hall edge channels in graphene quantum point contact. arXiv preprint arXiv:1605.08673, 05 2016.

[138] Jean Zinn-Justin. Critical phenomena: field theoretical approach. Scholarpedia, 5(5):8346, 2010. 

 \\ universite̊ PARIS-SACLAY

Titre : Quantification de la charge et criticalité quantique Kondo dans des circuits mésoscopiques avec peu de canaux

Mots clés : quantification de la charge; effet Kondo; criticalité quantique; renormalisation; universalité; non-liquide de Fermi.

Résumé : Cette thèse explore plusieurs sujets fondamentaux pour les circuits mésoscopiques qui incorporent un faible nombre de canaux de conduction électroniques.

Les premières expériences concernent le caractère quantifié (discret) de la charge dans les circuits. Nous démontrons le critère de quantification de la charge, nous observons la loi d'échelle prédite pour cette quantification ainsi qu'une transition vers un comportement universel à mesure que la température augmente.

Le second ensemble d'expériences concerne la physique critique quantique nonconventionnelle qui émerge du modèle Kondo multi-canaux. Par l'implémentation d'une impureté Kondo avec un pseudo-spin de valeur $1 / 2$ constitué de deux états de charge dégénérés d'un circuit, nous explorons la physique Kondo à deux- et trois-canaux. Au point critique quantique symétrique, nous observons les points fixes Kondo universels prédits, des exposants universels de lois d'échelle et nous validons les courbes complètes obtenues par le groupe de renormalisation numérique. En s'écartant du point critique quantique, nous explorons la transition depuis la zone critique quantique : par une visualisation directe du développement d'une transition de phase quantique, par l'espace des paramètres de la zone critique quantique ainsi que par les comportements d'universalité et d'échelle.

Title : Charge quantization and Kondo quantum criticality in few-channel mesoscopic circuits

Keywords : charge quantization; Kondo effect; quantum criticality; renormalization; universality; non-Fermi liquid.

Abstract : This thesis explores several fundamental topics in mesoscopic circuitries that incorporates few electronic conduction channels.

The first experiments address the quantized character (the discreteness) of charge in circuits. We demonstrate the charge quantization criterion, observe the predicted charge quantization scaling and demonstrate a crossover toward a universal behavior as temperature is increased.

The second set of experiments addresses the unconventional quantum critical physics that arises in the multichannel Kondo model. By implementing a Kondo impurity with a pseudo- spin of $1 / 2$ constituted by two degenerate charge states of a circuit, we explore the two- and three-channel Kondo physics. At the symmetric quantum critical point, we observe the predicted universal Kondo fixed points, scaling exponents and validate the full numerical renormalization group scaling curves. Away from the quantum critical point, we explore the crossover from quantum criticality: direct visualization of the development of a quantum phase transition, the parameter space for quantum criticality, as well as universality and scaling behaviors. 